\title{
Deciphering the details of RNA aminoglycoside interactions: \\ from atomistic models to biotechnological applications
}

by

\author{
Muslum IIgu \\ A dissertation submitted to the graduate faculty \\ in partial fulfillment of the requirements for the degree of \\ DOCTOR OF PHILOSOPHY
}

Major: Biochemistry

Program of Study Committee:

Marit Nilsen-Hamilton, Major Professor

W. Allen Miller

Gustavo Maclntosh

Ted W. Huiatt

Thomas A. Bobik

lowa State University

Ames, lowa

2012

Copyright @ Muslum Ilgu, 2012. All rights reserved. 


\section{TABLE OF CONTENTS}

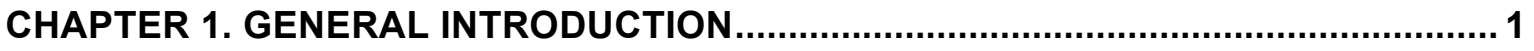

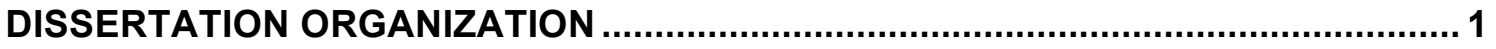

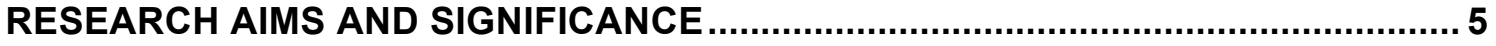

THE PRINCIPLE OF THE IMAGEtags REPORTER SYSTEM ................................. 7

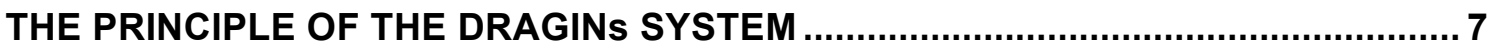

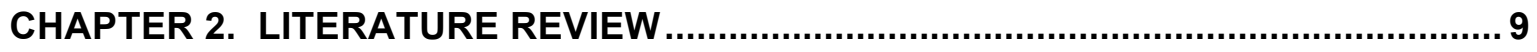

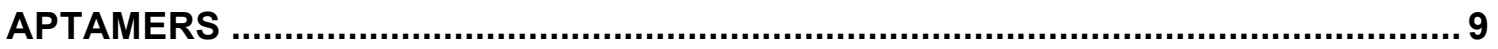

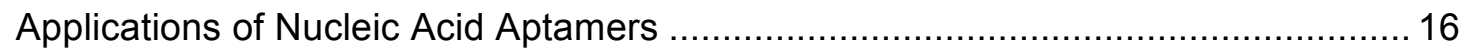

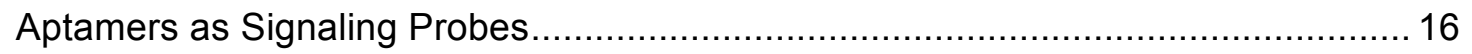

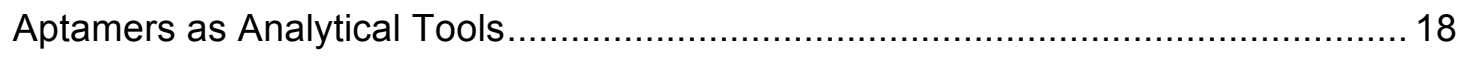

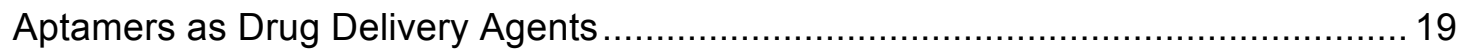

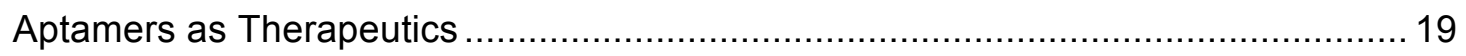

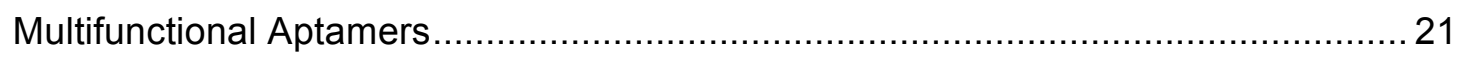

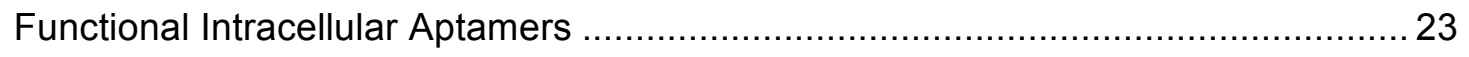

Aptamers to Control Transcription and Translation ...................................... 24

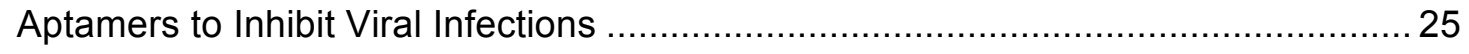

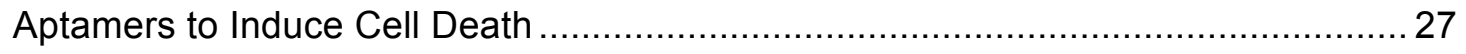

Aptamers to give Aminoglycoside Resistance to Bacteria ................................. 28

MOLECULAR MODELING OF NUCLEIC ACIDS AND APTAMERS ........................ 30

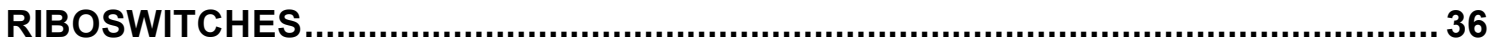

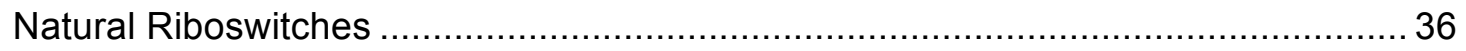

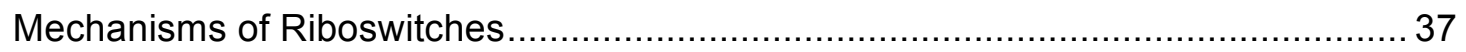




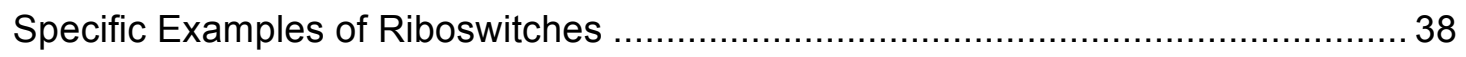

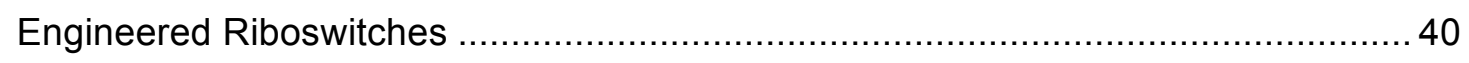

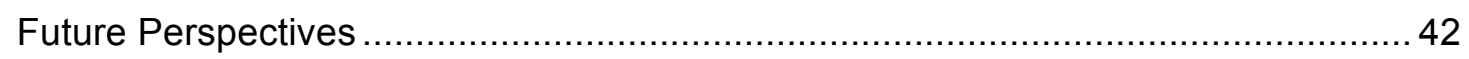

IMAGING TECHNOLOGIES AND RNA DETECTION........................................... 44

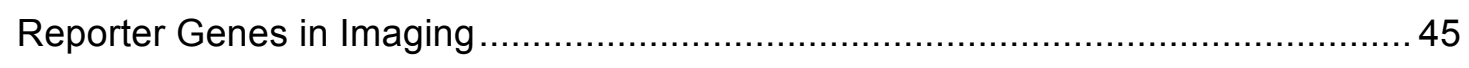

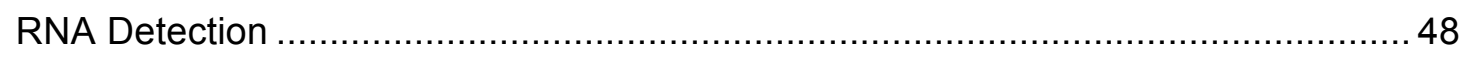

DRUG DEVELOPMENT AND RESISTANCE........................................................ 54

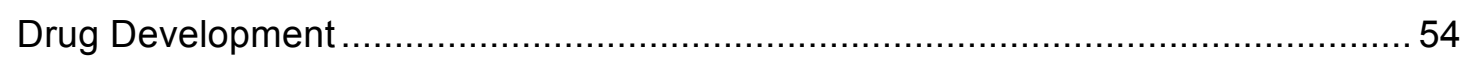

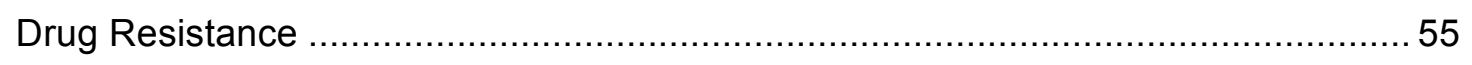

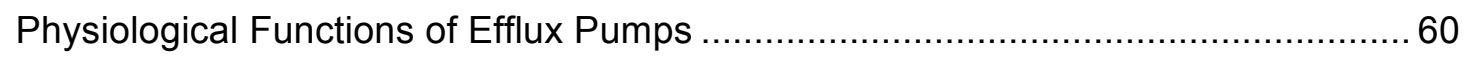

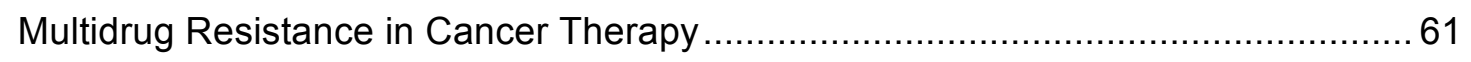

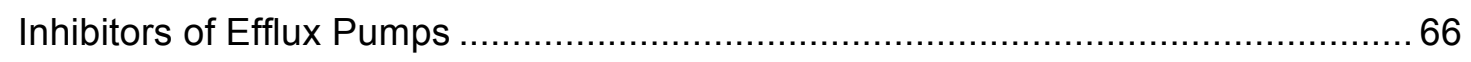

MECHANISMS OF RESISTANCE TO AMINOGLYCOSIDES ...................................71

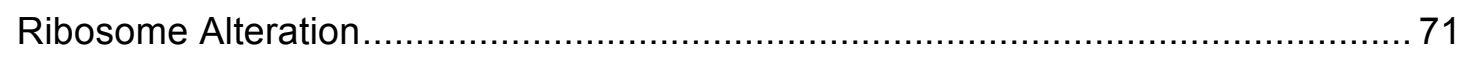

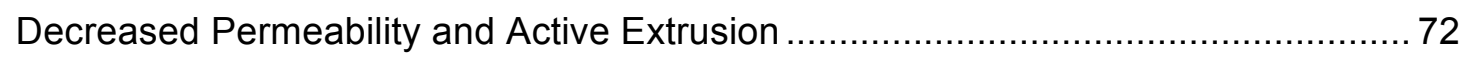

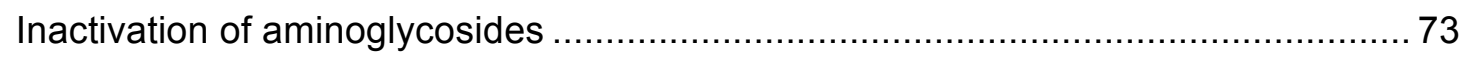

APPROACHES TO PREVENT DRUG RESISTANCE …......................................... 79

Simultaneous Use of Multiple Drugs ..................................................................... 79

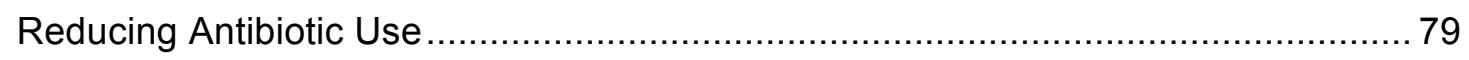

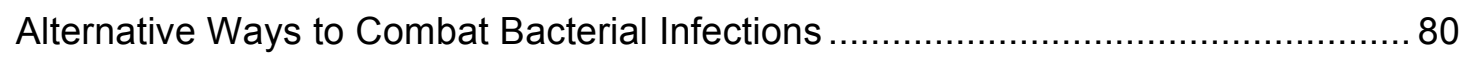

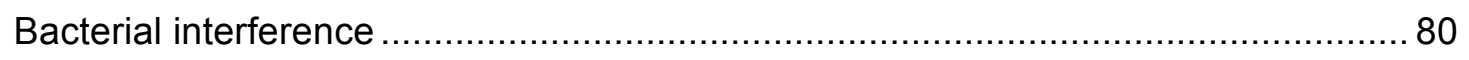

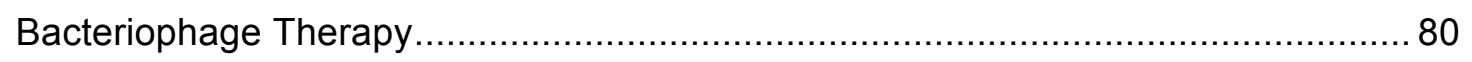

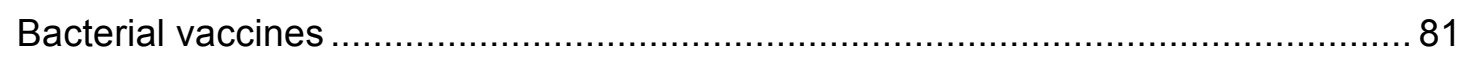

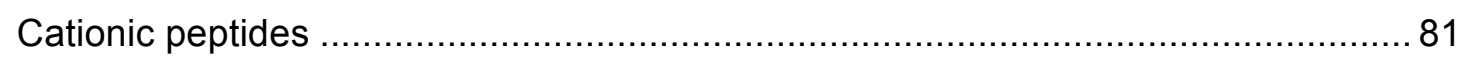




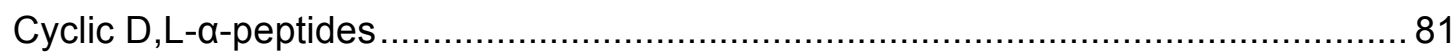

AMINOGLYCOSIDES AS INTRACELLULAR RNA APTAMER TARGETS ................ 83

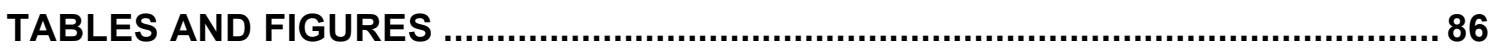

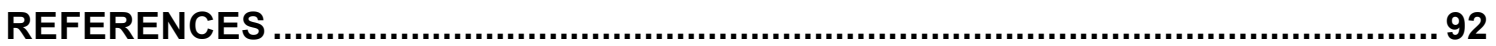

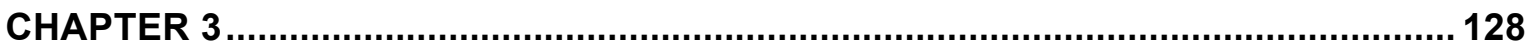

Factors that Influence Selectivity of the Neomycin-B RNA Aptamer......................128

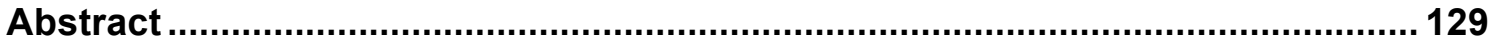

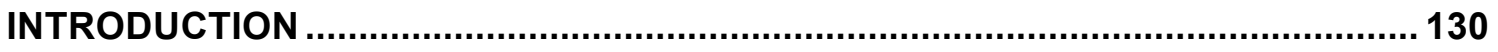

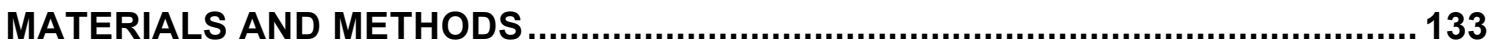

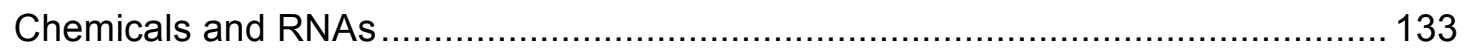

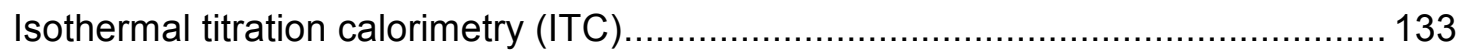

Molecular docking of aminoglycosides to the neomycin RNA aptamer via DOCK .. 134

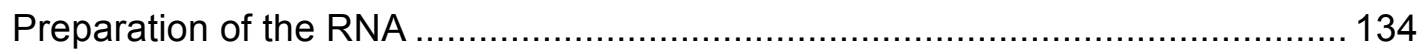

Preparation of aminoglycoside antibiotics ............................................ 134

Active site identification and molecular docking ....................................... 135

Post-docking analysis : Use of Ligplot to map the RNA-aminoglycoside

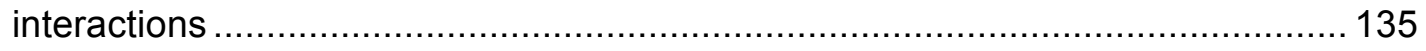

Measurement of 2-aminopurine (2AP) fluorescence intensity ......................... 136

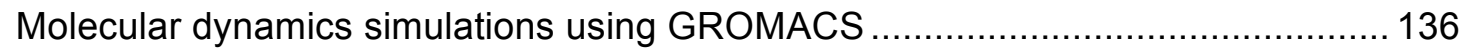

Preparing Neomycin-B for MD simulations ................................................ 137

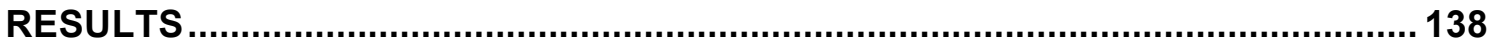

Broad aminoglycoside specificity of the neomycin-B aptamer ............................ 138

Molecular docking and MD simulations predict a hydrophobic interaction between

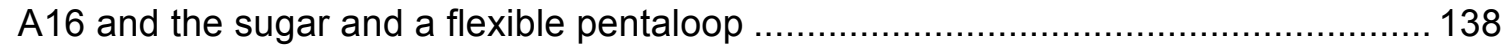


Experimental analysis of an aptamer with 2-aminopurine in position 16 identifies a shift in the environment of A16 with ligand binding. 139

Effects of buffer components on the aptamer-aminoglycoside specificity 140

The role of the A16 in establishing specificity of aminoglycoside binding. 141

DISCUSSION

ACKNOWLEDGEMENTS

REFERENCES

FIGURE LEGENDS

FIGURES AND TABLES

CHAPTER 4

DRAGINs: Drug Binding Aptamers For Growing Intracellular Numbers 162

Abstract 163

INTRODUCTION 164

Mechanism of action of the aminoglycosides ............................................. 166

Possible RNAs to use as DRAGINs that interact with aminoglycosides ................ 167

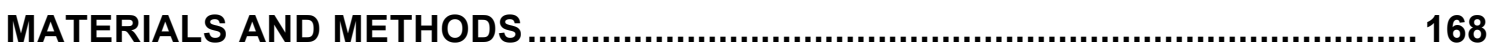

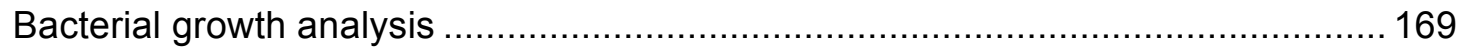

Analysis of binding interaction by isothermal titration calorimetry (ITC) ................ 169

Determination of IC50 values for aminoglycosides .................................. 170

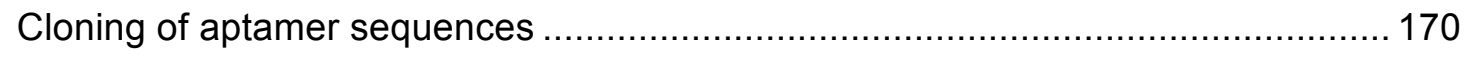

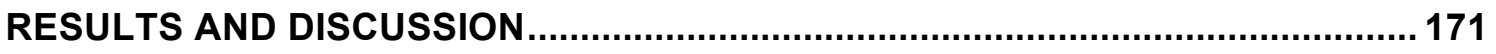

Decrease in IC50 for several different aminoglycosides was observed in cells expressing aptamers compared with controls 
Mathematical prediction I: Increase in drug concentration............................. 172

Measure extracellular drug concentrations .............................................. 172

Mathematical Prediction II: Mobility affects the aptamer action ......................... 173

FIGURE LEGENDS................................................................................ 174

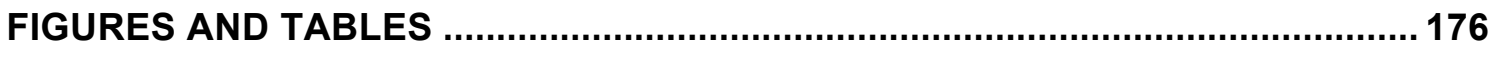

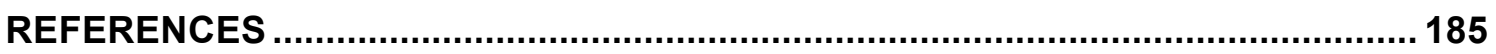

CHAPTER 5. GENERAL CONCLUSIONS......................................................... 189

THE SPECIFICITY OF AN RNA APTAMER IS HIGHLY DEPENDENT ON THE

IONIC ENVIRONMENT .............................................................................. 189

APTAMER-TARGET INTERACTIONS CAN BE UTILIZED FOR IN VIVO

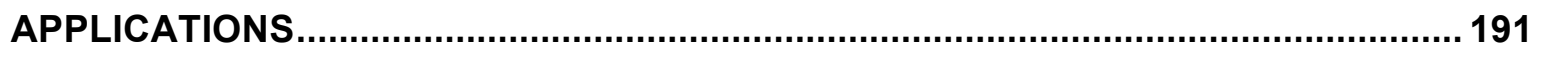

MATHEMATICAL MODELING OF APTAMER EXPRESSION MAY CHALLENGE

US TO IMPROVE THE DRAGINs SYSTEM .................................................... 191

UNDERSTANDING RNA-AMINOGLYCOSIDE INTERACTIONS MAY HELP TO

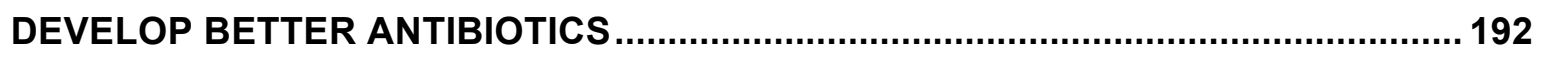

RNA-AMINOGLYCOSIDE INTERACTIONS MAY HELP US DEVELOP

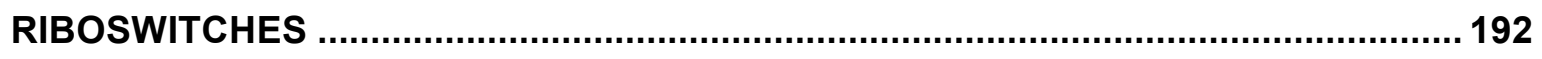

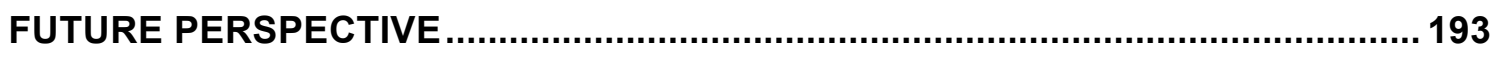

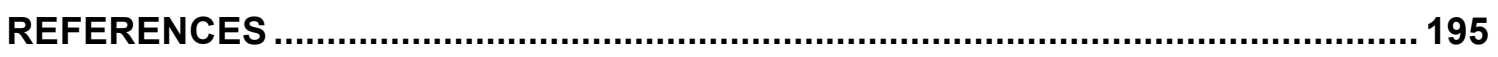

APPENDIX I. CONTRIBUTION to the DEVELOPMENT of IMAGEtags

(INTRACELLULAR MULTIAPTAMER GENETIC tags) for DETECTING PROMOTER

ACTIVITY IN LIVING CELLS ............................................................................ 196

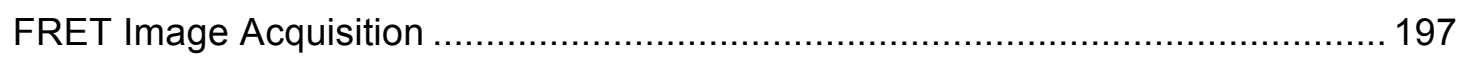

Test to check the stability of IMAGEtags ................................................. 198 


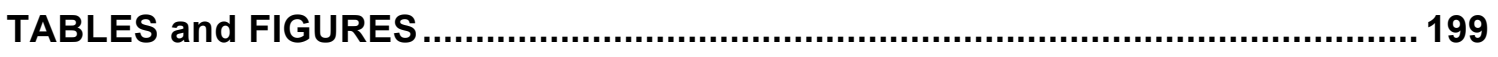

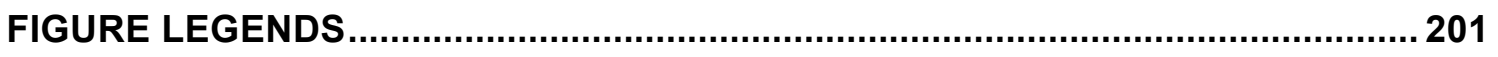

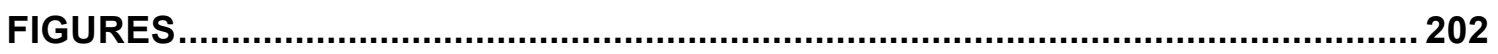

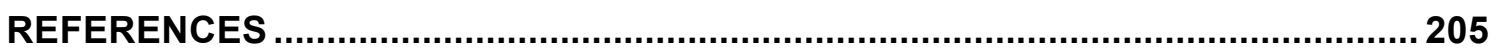

APPENDIX II. NUCLEAR MAGNETIC RESONANCE (NMR) STUDY OF THE NEOMYCIN-B RNA APTAMER TO DETERMINE THE CONFORMATION OF A16

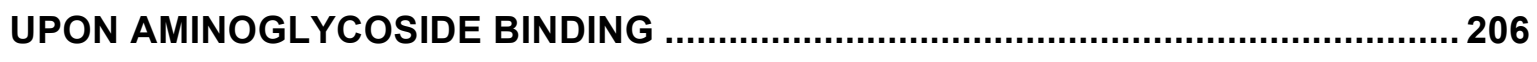

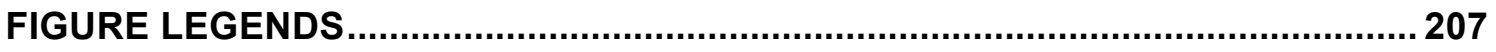

APPENDIX III. EQUILIBRIUM BINDING ANALYSIS OF RIBOSWITCHES THAT

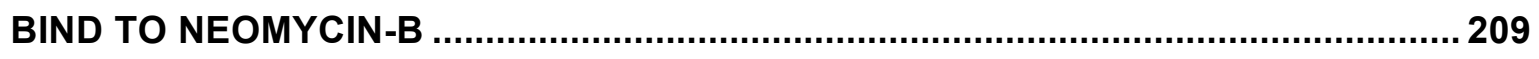

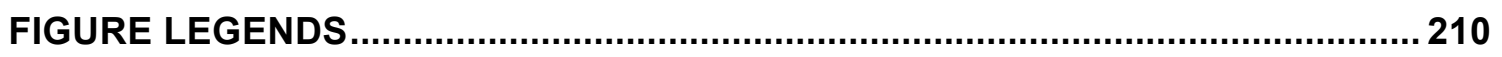

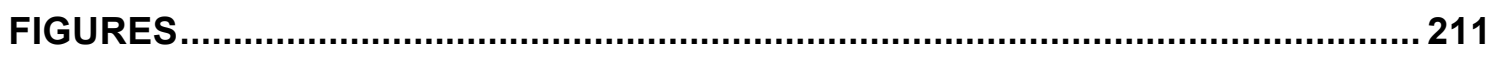

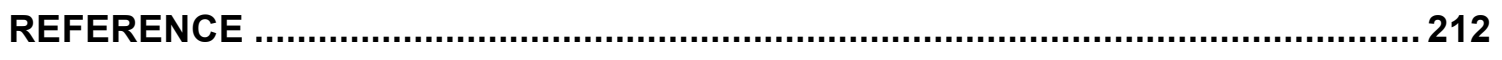

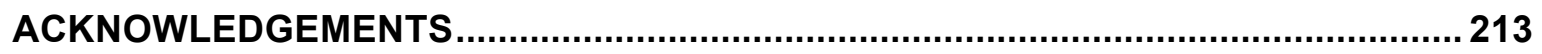




\section{CHAPTER 1. GENERAL INTRODUCTION}

\section{DISSERTATION ORGANIZATION}

This dissertation contains five chapters and three appendices.

Chapter 1 is a general introduction to the dissertation organization and the research significance of the studies included in this dissertation.

The second chapter is a literature review that discusses the aptamers and their comparison with antibodies (summarized in Table 1). Functional intracellular aptamers are then discussed to introduce the abilities of aptamers, which are strikingly different than those of antibodies. Following aptamers, multifunctional aptamers that are developed to increase potency and carry out multiple functions are discussed with some examples. Then, current imaging technologies and methods for RNA imaging are discussed to show their advantages and disadvantages. After the imaging technologies section, drug development is briefly introduced and followed by a drug development and resistance section in which mechanisms of drug resistance are discussed, mainly focusing on the efflux pumps. Also, mechanisms of resistance to aminoglycosides and approaches to prevent drug resistance are included in chapter 2. Then, aminoglycoside antibiotics are discussed as intracellular aptamer targets, how they can be utilized as ligands for the expressed RNA aptamers in a cell. Before the final section molecular modeling of nucleic acids, especially aptamers, is discussed. In the final section, riboswitches, which are RNA-based control units for gene expressions, are detailed. All together the literature review section aims to cover the related research that help guide the development of the studies carried out and presented in this dissertation.

Chapter 3 covers a detailed study of the neomycin-B RNA aptamer for determining its selectivity and binding ability to both neomycin- and kanamycin-class aminoglycosides. 
This manuscript is in preparation for submission to Nucleic Acids Research. Aminoglycosides are positively charged biomolecules, which make them very effective RNA targeting agents. Although they primarily target the $16 \mathrm{~S}$ rRNA decoding region (or A-site) and cause misreading of the genetic code, they have been found to interact with several other natural and artificial small RNAs. Our focus of study was a 23mer neomycin-B RNA aptamer. Previous analyses of the interaction of neomycin-B and paromomycin showed that the aptamer is highly neomycin-B specific (>100-fold higher affinity for neomycin-B than for paromomycin). However, our observations showed that paromomycin binds with only about 5-fold less affinity when the equilibrium was analyzed in solution by isothermal titration calorimetry (ITC). Therefore, we have re-evaluated the interactions of aminoglycosides with the neomycin-B RNA aptamer. Our data suggest a pattern for aminoglycoside binding as: neomycin-B > ribostamycin $\geq$ kanamycin-B $>$ paromomycin $>$ tobramycin $>$ sisomicin $>$ geneticin $>$ kanamycin-A > amikacin $\geq$ netilmicin. In parallel, this observation was supported by the docking results obtained using the NMR-resolved structure of the aptamer. The only outlier in the comparison was ribostamycin and so we further studied ribostamycin binding. After docking, the Ligplot analyses gave pictorial representations for each of the RNAaminoglycoside interaction with the residues in $\mathrm{H}$-bond and hydrophobic contacts. During docking, the RNA was treated as a rigid moiety, which limited our ability to simulate the atomic details of the interactions between the aptamer and aminoglycosides. Therefore, we performed molecular dynamics (MD) simulations to get more detailed information about the motion of each atom in the aptamer with and without neomycin-B. MD simulations showed that the adenine nucleotide at the $16^{\text {th }}$ position (according to the NMR numbering) changes its environment upon binding. The same residue was also found, as in the docking results, with a hydrophobic interaction between the aptamer and sugar. By contrast, it was found to have hydrogen bonds in the NMR structure. We experimentally validated this observation of 
change in microenvironment of A16 by performing fluorescence assays using 2AP-labeled aptamer at the $16^{\text {th }}$ position. Our results revealed that the A16 moves from a stacked position to more hydrophobic environment so that it gives more fluorescence upon aminoglycoside binding. Also, the increases in 2AP fluorescence differed for neomycin-B, ribostamycin and paromomycin suggesting that the A16 interacts with these ligands or with other moieties in the RNA differently depending on the ligand. Therefore, we tested the binding ability of A16 variants to the neomycin-family aminoglycosides and discovered that removing it or replacing it with other bases (uracil, cytosine or guanine) changed its aminoglycoside selectivity without affecting the neomycin-B binding. Finally, we found that the discrepancy in the selectivity of the aptamer was primarily due to a difference in the buffer environment from that which was used in the previous studies. To understand the impacts of individual buffer constituents on the specificity of the aptamer, we tested the binding interactions under five different buffer conditions, including the original buffer and the NMR buffer. Both the selectivity and affinity of the aptamer are highly buffer dependent. The aptamer becomes highly selective in cacodylate buffer that was used in the original study and binds poorly to paromomycin and ribostamycin. Finally, we have found that magnesium ions facilitate the aptamer adopting the proper $3 \mathrm{D}$ conformation for aminoglycoside binding and their presence decreases the entropic cost of the folding.

The contributions of the authors for the chapter 3 are described as follows. Muslum Ilgu designed the experiments, collected and analyzed data and wrote the manuscript. Molecular docking was carried out in collaboration with Taner Z. Sen, Raghu Yennamalli and Megan N. Kleckler. Raghu Yennamalli performed the docking and with Megan N. Kleckler they analyzed the results. MD simulations were carried out in collaboration with Monica H. Lamm and Megan N. Kleckler. Marit Nilsen-Hamilton worked with Muslum Ilgu to design the experiments, analyze the data and write the manuscript. 
In chapter 4, a novel method to increase drug concentrations in cells for more efficiently killing is described. To test the method, a bacterial model system was adopted and several small RNA molecules interacting with aminoglycosides were cloned downstream of T7 RNA polymerase promoter in an expression vector. Then, the growth analysis of E. coli expressing aptamers was observed for 12-hour period. Our analysis indicated that aptamers helped to increase the intracellular concentration of aminoglycosides thereby increasing their efficacy.

The research work presented in chapter 4 is in preparation and the authors for it are described as follows. Muslum Ilgu designed experiments, collected and analyzed data and wrote the manuscript. Robert Feldges collected data, Supipi Liyamali Auwardt designed experiments, collected and analyzed data. Marit Nilsen-Hamilton worked with Muslum Ilgu to design experiments, analyze the data and write the manuscript.

Chapter 5 is the general conclusion of the studies addressed in chapters 3 and 4 . This chapter summarizes the importance of binding studies of aminoglycosides with small RNA molecules for deciphering the residues responsible for these interactions. Other topics of discussion in chapter 5 are future directions and potential applications of the insights achieved in chapter 3 and chapter 4 . Finally, a perspective of aptamer research in general is discussed.

Appendix I describes Muslum Ilgu's results for developing an RNA-based imaging technique for visualization of gene expression in vivo and in real time. This work has been submitted to Nature Methods, and the development of IMAGEtags (Intracellular MultiUptamer GEnetic tags) for visualizing gene expression in mammalian cell cultures. For this purpose, the collaboration continues with Ilchung Shin, Judhajeet Ray and Samir Mehanovic from Marit Nilsen-Hamilton's lab, Jonathan Beasley from George Kraus's lab at 
lowa State University in the Department of Chemistry and Ming Zhao and Leilei Peng at University of Arizona in Optical Sciences.

Appendix II describes the preliminary NMR results that were performed to understand the conformation of $16^{\text {th }}$ residue upon aminoglycoside binding. This work is in progress and once the assignments are completed in collaboration with Bruce Fulton, NMR facility director at lowa State University in BBMB department, it will be included in chapter 3 to complete the manuscript. ${ }^{1} \mathrm{H}$, NOESY and TOCSY NMR experiments have been performed so far and the results are presented in Appendix II.

Appendix III describes Muslum Ilgu's results from experiments designed with the purpose of understanding the aminoglycoside selectivity of three different neomycin-B riboswitches that are used to regulate gene expression. The results indicated that these neomycin riboswitches are also able to interact with a variety of aminoglycosides. Future work will involve testing them in vivo with more than neomycin-B to determine if they are responsive to other aminoglycosides.

\section{RESEARCH AIMS AND SIGNIFICANCE}

Aminoglycosides are natural antibiotics used for many years to combat bacterial infections. Because new strains of bacteria with antibiotic resistance emerge each year, the antibiotics currently in the market are expected to eventually lose their effectiveness. Therefore, new antibiotics or other ways of combating pathogens are needed to solve this antibiotic resistance problem. Combining new approaches to understand the details of RNA-aminoglycoside interactions can help us develop new more effective antibiotics.

The higher order structures and flexibility of the aptamers allow them to interact with more than one type of a target such as with a variety of aminoglycosides as discussed in chapter 3. Better understanding of the regulation of aptamer structure and dynamics 
through MD simulations is essential for more rapidly and effectively developing aptamer applications as well as improving their features like target specificity and affinity. This was achieved by determining the binding affinities and the dynamics of the RNA-aminoglycoside complex, then testing the predictions with wet-lab experiments. These findings and the experimental approaches taken to obtain data can help researchers to modify the present drugs to enhance their effectiveness.

An alternative way to developing new drugs is to discover new techniques to increase the efficacy of present antibiotics. Development of such methods is necessary due to the emergence of drug resistant strains. A challenge in cancer treatment is to maintain a high enough intracellular drug concentration to kill the cancer cells. This is because cancer cells upregulate the expression of multi-drug transporters that leads to the need for higher extracellular concentrations of drugs to compensate for a counter balancing activity that promotes cellular entry of the drugs for effective cell killing. Modern drug synthesis research has addressed this issue by many means, mainly based on "push mechanisms". However, the extent to which the extracellular drug concentration and the cell permeability to the drug can be increased is limited. Another way to increase intracellular drug concentrations is for the cells to express short RNA aptamers that can bind to their drug target. Aptamers behave like antibodies but have more dynamic structures and can function in a cell. We have named these aptamers DRAGINs (ㅁug Binding Aptamers for Growing Intracellular Numbers). To test this hypothesis experimentally, we used bacteria that are sensitive to killing by aminoglycosides (the drugs) and aminoglycoside aptamers (DRAGINs) to increase cellular sensitivity to the drugs. These features were utilized as described in chapter 4 in detail to increase aminoglycoside concentrations in E. coli as a proof of principle. The same concept can later be applied into the cancer cells. 
RNA aptamers can be utilized for other applications in vivo. One way is to use them as real time imaging platforms. This may lead us to understand the real time dynamics of gene expression at the promoter level. The development of this method involves the labeling of aptamer targets, aminoglycosides, which can go through the cellular membrane and bind to aptamers once they are expressed. In addition, increasing the number of expressed aptamers in a string can increase the signal/noise. Adding the use of fluorescence resonance energy transfer (FRET), which occurs if the donor and acceptor dyes are brought close to each other with expressed IMAGEtag aptamer, can increase the validity of the signal. This method is still under development using several different aptamers and their labeled targets. The progress of this project is explained in the Appendix I.

\section{THE PRINCIPLE OF THE IMAGETAGS REPORTER SYSTEM}

The IMAGEtag (intracellular multi aptamer genetic tag) consists of a string of multiple tandem aptamers that can be expressed in the cells as RNA. The IMAGEtag does not encode a protein, but could be part of a fusion construct with endogenous mRNAs so that these can be localized and tracked in the cell. Detection of the IMAGEtags relies on the aptamers' binding to a ligand molecule that is labeled with a fluorescent moiety or radioactive element, which would be added to the cells before analysis. To increase the binding capacity and the potential signal of such tags, constructs with several aptamers have been created by employing a cloning technique based on methods previously used for creating repetitive polypeptide units.

\section{THE PRINCIPLE OF THE DRAGINS SYSTEM}

The test of a new approach based on a "pull mechanism" by expressing molecules that can bind drugs inside the cells and increase their intracellular concentrations was 
achieved in DRAGINs system. The expressed aptamers selected for tight and specific binding to their drug targets are aminoglycoside-binding RNAs. It was predicted that the presence of mobile aptamers inside the cells would increase the intracellular concentration of the target drug compared to in the absence of aptamers by mathematical modeling. In this context, the intracellularly expressed aptamers are referred to as DRAGINs (Drug Binding Aptamers for Growing Intracellular Numbers). To test this hypothesis experimentally, we used bacteria that are sensitive to killing by aminoglycosides (the drugs) and aminoglycoside-binding RNA aptamers to increase cellular sensitivity to the drugs. $E$. coli BL21 strain was transformed with aptamer-expressing plasmids and incubated with different concentrations of aminoglycosides to determine the effect of aptamer expression on efficacy of cell killing. 


\title{
CHAPTER 2. LITERATURE REVIEW
}

\begin{abstract}
APTAMERS
Ribonucleic acid (RNA) molecules contain only four types of bases; adenine (A), cytosine $(C)$, guanine $(G)$ and uracil $(U)$. Despite their simple composition, RNAs have diverse biological functions. It has long been believed that RNA is mainly a genetic information carrier in the cell as in the form of mRNA, and/or tRNA. However, in recent years, some complex roles of RNA molecules have been clearly demonstrated [1]. Many RNAs provide structural support for molecular machineries. Also, in the early 1980s the discovery of RNA's catalytic capability led to the speculation of an "RNA world" that preceded the advent of DNA and protein [2, 3]. Likewise, small RNAs have been shown to be very critical in regulating gene expression via modulation of translation initiation and mRNA stability [4]. However, until the end of $20^{\text {th }}$ century, very little was known about the principles underlying higher order RNA structures and their interactions with other molecules.

'Aptamer' (from the Latin, aptus, meaning 'to fit') is a single stranded nucleic acid (DNA or RNA) molecule that can bind to its target with high specificity and affinity. Although the term has also more recently been applied to peptides with similar characteristics that are called peptide aptamers, this study focuses on nucleic acid aptamers as they are utilized to develop new biotechnological tools. In 1990, two laboratories independently developed the technique of aptamer selection: the Gold lab used the term 'SELEX' (Systemic Evolution of Ligands by EXponential enrichment) for their process of selecting RNA ligands against T4 DNA polymerase [5] and the Szostak lab coined the term 'in vitro selection' for selecting RNA ligands that could bind to various organic dyes [6].

Since the discovery of the aptamer selection process, various uses of aptamers have been documented in the literature. Researchers have identified numerous high affinity
\end{abstract}


aptamers that target a broad range of molecules. Many aptamers have been selected in vitro and optimized to bind to various molecular targets such as small molecules, proteins, nucleic acids, even cells, and tissues. Some of these RNAs have found their way for both in vitro and in vivo applications [7-11]. In 2006, an aptamer handbook was published to describe various aspects of aptamer research, development and application [12]. Also, an aptamer database has been created to collect various aptamers selected in vitro through SELEX (http://aptamer.icmb.utexas.edu/) [13].

Nucleic acid aptamers are expected to be very helpful tools in the fields of therapeutics, drug development, target validation and diagnostics as they offer molecular recognition properties that rival that of the commonly used biomolecules, antibodies. An aptamer can discriminate a single chemical group difference between two molecules or even the same molecules with different conformations [14-16]. In addition to their discriminatory recognition, aptamers offer advantages over antibodies as they are lower cost and possess desirable storage properties. Furthermore, they elicit little or no immunogenicity in therapeutic applications [17-21]; whereas antibodies, even humanized ones, can induce immunogenicity [22]. Another advantage of using SELEX is that aptamers can be selected against toxic compounds, which would be otherwise hard to target.

The SELEX process usually takes about 8 weeks for the selection but the additional optimization required for many aptamers results in it taking more time to develop an aptamer than to obtain an antibody. However, the main disadvantage of antibody selection is that it needs animals for antibody production and each time a new batch of antibody is produced it must be optimized for its applications. In contrast, one can get aptamers, in the test tube, with binding equilibrium constants in the $\mathrm{pM}$ to $\mu \mathrm{M}$ range, which often compares favorably to the binding strength of many antigen-antibody complexes [23]. In addition to their potential use as pharmaceutical and diagnostic agents, another advantage of aptamers is that they 
function both inside and outside cells [24-28]. Whereas, antibodies must be added extracellularly, aptamers can be produced intracellularly. For these reasons, aptamers are competitive with or even superior to antibodies in many respects as summarized in Table 1.

SELEX is an iterative process of adsorption, recovery and re-amplification. It begins with a large synthetic pool (library) of single-stranded nucleic acids, which is challenged to bind either to a purified protein, or a small peptide, a small molecule, a nucleic acid or a whole cell. The starting SELEX population has fixed ends for PCR primerannealing. One of the ends contains T7 RNA polymerase promoter for in vitro transcription by which RNA aptamers can be enriched in SELEX. Between these fixed ends, combinatorial libraries often contain 30 to 40 nucleotide-long contiguous random sequences otherwise known as sequence space. The theoretical diversity of such a combinatorial library can potentially encompass $4^{30}\left(10^{18}\right)$ or $4^{40}\left(10^{24}\right)$ individual sequences available for partitioning. However, this number often exceeds, by many orders of magnitude, the number of molecules that can be handled in practice. So, convenience and cost generally restrict the starting pool to a sample of no more than $10^{12}-10^{15}$ randomly chosen oligonucleotides, each of which is presumed to contain a unique base sequence. However, it has been claimed that even less diverse combinatorial libraries will yield useful aptamers $[29,30]$.

The desired property of an aptamer is, typically, the ability to bind to a given ligand with high affinity and selectivity. Thus, for a successful SELEX experiment, it is crucial to identify an effective method for partitioning. By mixing the solution-phase library under defined conditions with the target molecule and subsequently retrieving the target and removing the unbound or loosely bound nucleic acids, the few nucleic acids that have bound to the target can be recovered from the library. To achieve this partitioning, nitro-cellulose filter binding, affinity chromatography, size fractionation on columns, flow cytometry, surface 
plasmon resonance (SPR), electrophoresis on native polyacrylamide gels (PAGE) or capillary electrophoresis (CE) can be used.

To select the highest affinity binding molecules, low binding-affinity molecules need to be selected against by sequential rounds of selection. At each round, the selected nucleic acid molecules are subsequently amplified as DNA. If the single-stranded library is of RNA or RNA analogs, it is first copied into complementary DNA (cDNA) by reverse transcriptase (RT). These single-stranded DNA molecules are amplified directly by PCR. The DNA population is subsequently used to generate a new library of single-stranded molecules (in vitro transcription for RNA or strand separation for DNA), which is subjected to another round of selection procedure. This process is repeated until a population of highaffinity single-stranded molecules is selected. Because the affinity-based partitioning methods are imperfect, the cycle of partitioning and amplification is normally repeated 6 to 12 times or more, until molecules of the desired property predominate in the population of nucleic acids. In the final step, the binders are cloned and sequenced for further analysis and characterization.

The process of SELEX achieves a reduction in sequence diversity from the initial sample of sequence space to a population that is typically dominated by about 10 distinct sequences with appreciable binding to the desired target. In addition, the error-prone nature of the enzymes used to amplify the selected sequences combined with high concentrations of dNTPs and $\mathrm{Mg}^{+2}$ favor the introduction of random mutations that effectively allow the procedure to sample a greater proportion of the sequence space than was initially sampled. A process very similar to Darwinian evolution by natural selection and survival of the fittest is the explanation for the appearance of clearly related, but evolutionarily divergent, sequences in the final aptamer population [31]. Overall, the combination of PCR, in vitro transcription, and isolation of the bound sequences in each round usually takes several days; 
therefore, high-affinity aptamers can be enriched and successfully isolated in 8 to 10 weeks through conventional SELEX.

During the SELEX process, depending on the anticipated application, the desired binding properties can be adjusted to be a fast association rate, slow dissociation rate, high affinity, low affinity to closely related molecules, or a combination of all. In order to get higher selectivity, negative selection can be deployed against the partitioning matrix, or a structural analog of the target by which non-specific binders are eliminated. Also, for high affinity, the target concentration can be limited at the beginning of the SELEX and gradually decreased in the later rounds. As a result, at the end of the SELEX process, the enriched population of aptamer sequences can have both high specificity and high selectivity to the desired target.

Aptamer-target interaction is mainly determined by the tertiary structure of the folded nucleic acid aptamers. The three-dimensional structure of an aptamer is uniquely determined by the sequence of its bases. When an aptamer is bound to its ligand, there will possibly be van der Waals surface contacts, hydrogen bonds, stacking interactions and other noncovalent bonds that make the interaction highly specific and functional. This very high specificity was exemplified with the theophylline aptamer [14]. Initially, five rounds of regular selection were performed by capturing the RNAs on a sepharose column derivatized with 1-carboxypropyl theophylline. This initial selection was followed by another three rounds of counter-selections performed against caffeine, structurally differs from theophylline with an additional methyl group. The resulting pool contained 15 conserved bases and out of this pool, a 42 nucleotide aptamer (TCT8-4) was found to have high affinity for theophylline with a dissociation constant of $0.32 \mu \mathrm{M}$, which was shown 10000 -fold higher than for caffeine (Jenison et al., 1994). 
On the other hand, for some targets the aptamer's specificity can be broad depending on the nature of the chemical interactions that dominate the interaction of these aptamers with their targets. For example, different aminoglycoside antibiotics were demonstrated to bind the lividomycin and kanamycin-B RNA aptamers. In the case of the former, most of the aptamers originally selected to bind to lividomycin were found to be able to interact with both neomycin-B and paromomycin, as well [32]. Similarly, kanamycin-B aptamers initially selected to recognize kanamycin-B were later found to be able to even better interact with tobramycin than kanamycin-B [33]. This lack of specificity was probably due to the predominance of electrostatic interactions between the aminoglycosides and the RNA aptamers. In fact, it was clearly demonstrated for neomycin-B RNA aptamer that electrostatic interactions and hydrogen bonding dominate the interactions between neomycin-B and its aptamer and are mainly due to the positively-charged nature of neomycin-B [34].

The omnipresence of degrading enzymes in virtually all biological fluids is a potential problem due to aptamers consisting of biopolymers of natural origin. Nucleic acids are degraded by nucleases in blood within seconds to a few minutes [35, 36]. Therefore, aptamers that are utilized in biological fluids need chemical modifications in order to make them more stable. Several strategies have been developed by which the potential targets for the degrading enzymes are modified for enhancing aptamer stability. The first examples included the use of nucleotides with 2'-OH position of the ribose modified with 2'-deoxy, 2'amines (2'- $\left.\mathrm{NH}_{2}\right)$ and 2'-fluorines (2'-F) [37] and later with (2'-OME) [38]. RNA aptamers with 2'- $\mathrm{NH}_{2}$ and 2'-F have increased stability and enhanced biological half lives [39-41]. RNAs can also be modified by covalently linking the $2^{\prime} \mathrm{O}$ and $4^{\prime} \mathrm{C}$ with a methylene group and obtaining so called 'locked nucleic acids' (LNA). Thermostability, tumor uptake, blood 
retention and plasma stability of tenascin-C binding aptamer were increased with the LNA modification [42].

Another way to get nuclease-resistant aptamers is the use of L-nucleic acids which cannot be recognized by endonucleases. Thus, researchers have obtained nucleaseresistant and plasma-stable 'spiegelmers' (from the German word Spiegel, mirror) with Lnucleic acids in place of natural D-nucleic acids [43-45]. Nevertheless, chemical synthesis of the mirror-image targets limits the number of available spiegelmers. The 4'-thio (-S) modification is another way to give nuclease resistance to aptamers. By utilizing 4'-thioCTP and 4'-thioUTP during SELEX to select a human $\alpha$-thrombin aptamer the half life was enhanced from $22 \mathrm{~min}$ to $19.5 \mathrm{~h}$ in the presence of $50 \mathrm{pg} / \mu \mathrm{l}$ RNase A [46].

Replacing one or two of the nonbridging oxygens on the phosphodiester backbone with thiol (-S) groups was also shown to provide nuclease resistance for aptamers. The half life of the thio-aptamer against NF-IL6 was increased four-fold in the presence of $0.12 \mathrm{pg} / \mathrm{ml}$ DNase I when compared to the unmodified NF-IL6 aptamer [47]. By the same token, capping the 5' and 3'-termini of aptamers enhances nuclease resistance because the degradation of aptamers in human blood is largely due to $3^{\prime} \rightarrow 5^{\prime}$ exonucleases. An inverted thymidine (3'-idT) is commonly used to cap the 3 ' end which creates an oligonucleotide with an two 5'-ends and no 3'-end. Combined with the previously-mentioned 2'-F and 2'-OME ribose modifications, 3'-idT increased the half-life of a platelet-derived growth factor (PDGF) DNA aptamer from $0.6 \mathrm{~h}$ to $8 \mathrm{~h}$ in $85 \%$ rat serum [48]. Short phosphorothioate caps were also used at the 5' and 3' ends to improve aptamer stability [49]. Also, researchers obtained multivalent circular DNA aptamers with both thrombin and L-selectin aptamers by connecting the 5' and 3' ends [50]. The circular RNA aptamer for streptavidin was generated both in vitro [51] and in vivo [52] through self-ligating permutated intron-exon 
sequence. These modifications helped the successful development of aptamer applications for use in human fluids.

\section{Applications of Nucleic Acid Aptamers}

The versatility of nucleic acids is due to their ability to display distinct and stable tertiary structures. Thus, they are able to form binding pockets and catalytically active centers. The SELEX procedure is now generally recognized as a powerful tool for selecting high-specificity and high-affinity nucleic acid aptamers for almost any target molecule. Aptamers have been used for a wide range of in vitro and in vivo applications $[8,10,11,53-$ 60]. Target molecules in the SELEX range from small molecules like ATP [30, 61], sulforhodamine B [62], theophylline [14], amino acids [15, 63, 64], carbohydrates [65], aminoglycoside antibiotics [33, 66, 67], citrulline [68] and vitamins [69] to all kinds of proteins including enzymes [70, 71], transcription factors [72-74], viral proteins [75-80], cell adhesion proteins [81-83], growth factors [84-86] and cytokines [87]..

\section{Aptamers as Signaling Probes}

Fluorescent modification before or after during SELEX has been used to obtain signaling aptamers. One type of signaling aptamer is called a 'aptamer beacon', which is modified with a quencher and a fluorophore at the 5' and 3' ends respectively. Interaction of ligand with the aptamer causes conformational changes that result in the fluorophore and quencher changing their relative positioning, thereby changing fluorescence. An antithrombin aptamer was engineered into an aptamer beacon by adding nucleotides to the $5^{\prime}-$ end, which are complementary to nucleotides at the 3 '-end of the aptamer, to form a duplex so that the attached fluorophores did not affect the thrombin binding site [88]. Upon thrombin binding, the conformational change was followed by an increase in fluorescence. 
This exemplifies the aptamer beacon as a sensitive tool for detecting proteins through changes in fluorescence.

Simpler fluorescent aptamers have only one fluorophore, which changes its fluorescence intensity due to the structural changes of aptamers upon their ligand binding [59]. Blank et al used a FITC- ${ }^{18} \mathrm{C}$-conjugated DNA aptamer, selectively binding to tumor micro-vessels but not normal blood vessels, to visualize a rat brain tumor by fluorescence microscopy [89].

Another type of signaling aptamer is the 'structure-switching signaling aptamer' [90]. In this case, the duplex is formed between a fluorophore-labeled aptamer and a small oligonucleotide modified with quencher, which hybridizes with the binding site of the aptamer. When the ligand binds, the hybridized oligonucleotide with quencher is released and the fluorophore emission can be detected.

In addition, some aptamers were designed to generate signals based on fluorescence resonance energy transfer (FRET) $[88,91]$. In the recent article, a sensitive method for rapid angiogenin detection based on FRET has been described. A dual-labeled angiogenin aptamer probe was constructed by labeling the $5^{\prime}$ with 6carboxyfluorescein (FAM) and 3' with 6-carboxy-tetramethylrhodamine (TMR) as donor and acceptor, respectively. By monitoring the fluorescence intensity of donor and acceptor on this aptamer, the quantitative detection limit was 200 pM angiogenin and the linear range was between $500 \mathrm{pM}$ to $40 \mathrm{nM}$ angiogenin [91].

Finally, aptamers are functionalized with gold nanoparticles (GNPs) that can be used for computed tomography (CT) imaging [92]. Compared with nanoparticles, small iodinated molecules normally used in CT have the following limitations; a lack of targeted molecular imaging, short imaging time, and renal toxicity. However, by functionalizing the surface of gold nanoparticles with an RNA aptamer specific to prostate-specific membrane antigen 
(PSMA), these limitations were eliminated. At the same time, a targeted molecular CT imaging system was enabled capable of specifically imaging prostate cancer cells that express the PSMA protein. Their results indicated that PSMA aptamer-conjugated GNP has more than 4-fold greater CT intensity for a targeted LNCaP cell than that of a nontargeted PC3 cell [92].

\section{Aptamers as Analytical Tools}

Aptamers also hold great potential as analytical tools in separation and molecular recognition technologies [57, 93]. For example, the thyroid transcription factor 1 (TTFI) aptamer was biotinylated and attached to streptavidin magnetic beads to purify TTFI [94]. The affinity chromatography purification assay on protein mixtures from bacteria lysates showed good specificity for TTFI. In another example, thrombin was specifically detected through MALDI-TOF-MS when the thrombin aptamer was immobilized to the matrix surface [95]. In the proximity-dependent DNA ligation assay, a long single stranded DNA (ssDNA) attached to the platelet-derived growth factor (PDGF) was ligated when the aptamers were brought close together on the PDGF dimer and then amplified to detect zeptomolar concentrations of the PDGF dimer [96].

Modular aptameric sensors have been developed utilizing the fluorescence increase from malachite green (MG) after it is bound by its RNA aptamer [97]. Using rational design, the ATP RNA aptamer, theophylline RNA aptamer or flavine mononucleotide (FMN) RNA aptamer, separately were fused with the truncated MG RNA aptamer. The presence of adenosine, theophylline or flavine mononucleotide (FMN) could be detected by an increase in the fluorescence of malachite green. In another sensor, the cocaine aptamer was used as part of a colorimetric probe for cocaine using the dye, diethylthiotricarbocyanine iodide. 
Cocaine displaced the dye from the aptamer, which resulted in its decreased absorbance, possibly due to its precipitation [98].

\section{Aptamers as Drug Delivery Agents}

Aptamers can also be used as delivery vehicles for drugs or signaling molecules either when they are linked covalently or indirectly through a hybridized oligonucleotide to the drugs. For example, an irreversible inhibitor of neutrophil elastase, valine phosphonate (ValP), was linked to an oligonucleotide hybridized to a random DNA/RNA library. In vitro selection generated an aptamer-ValP complex with increased potency by three to four orders of magnitude and with better specificity compared to ValP alone $[99,100]$. A weak inhibitor of human neutrophil elastase (hNE), a tetrapeptide Ala-Ala-Pro-Val, was covalently linked to the 3 '-end of a hNE aptamer with three 18-carbon linkers through post-SELEX modification. By this way, the aptamer could deliver the tetrapeptide to the active site of hNE and increase the drug potency by $10^{5}$ times compared to the drug alone [101].

Aptamers were also functionalized with gold nanoparticles (GNPs) for combined computed tomography (CT) imaging and prostate cancer therapy as explained earlier. In the same report, Kim et. al. showed that the PSMA aptamer-conjugated GNPs were significantly more potent against targeted LNCaP cells than against nontargeted PC3 cells after loading of doxorubicin [92]. By this means, they successfully delivered the anti-cancer drug, doxorubicin, specifically to the targeted cells.

\section{Aptamers as Therapeutics}

The idea of using aptamers as therapeutic agents is now more than 20 years old and noticeable progress has been made over time. Aptamers have been developed as anticancer, anti-microbials, anti-coagulation, anti-inflammation, anti-angiogenesis, antiproliferation and immune therapy [7, 10]. For example, there are aptamers developed as 
anti-viral therapeutics using the selected aptamers against HIV-1 Rev [102], HIV-1 integrase [103] and HIV-1 reverse transcriptase [104]. These anti-HIV aptamers are discussed in the functional intracellular aptamers section. In this section, anti-cancer, anti-angiogenesis and anti-coagulation aptamers are discussed as therapeutic applications of aptamers.

Aptamers can be used in cancer therapy with inclusion of boron neutron capture therapy. Boron $\left({ }^{10} \mathrm{~B}\right)$ can capture neutrons to form ${ }^{11} \mathrm{~B}$, which can then split into ${ }^{4} \mathrm{He}$ and ${ }^{7} \mathrm{Li}$ and kills cells within a distance of 5-9 $\mu \mathrm{m}$. Therefore, aptamers consisting of boronated nucleotides will gain new functionalities for cancer therapy if the aptamers are targeted to cancer-cell markers. Aptamers against ATP have been selected using either 5'-( $\alpha$-Pborano)-UTP or 5'-(a-P-borano)-GTP during SELEX. The selected aptamers showed novel folding and the bU and bG were essential for aptamer function [105].

Aptamers were also developed as anti-coagulation agents. Thrombin, a serine protease converted from pro-thrombin by the action of factor $\mathrm{Xa}$, is a key regulatory enzyme in the coagulation pathway by converting fibrinogen into fibrin [106]. 2'-fluoro-modified RNA molecules were exposed to a mixture of human and porcine a-thrombin in round 1 and then toggled between human and porcine thrombin in alternate rounds of selection [107]. An aptamer named Toggle-25 was identified that bound to human thrombin with an affinity of $2.8 \mathrm{nM}$ and to porcine thrombin with $\sim 83 \mathrm{pM}$ affinity. An aptamer that recognized both human and porcine thrombin could be developed further in a porcine model system before being tested in human clinical trials. Further characterization showed that the aptamer could increase the thrombin life-time by 2 -fold in human plasma and by 4 -fold in porcine plasma. Also, the aptamer inhibited thrombin-dependent platelet activation in a dose-dependent manner in both human and porcine platelets [107].

At the end of 2004, there was a breakthrough in the therapeutics application of aptamers when the Food and Drug Administration (FDA) approved the first aptamer drug 
called Macugen by OSI Pharmaceuticals or pegaptanib sodium by Pfizer and Eyetech. This drug was developed as a potent angiogenesis inhibitor to recognize vascular endothelial growth factor (VEGF) and is used to treat wet age-related macular degeneration (AMD) [86, 108]. The 27 nucleotide aptamer includes 2'F pyrimidine, 2'OME purine, 5'-end PEG and also a 3'-end dT modifications to protect it from endonucleases. These modifications stabilize the aptamer in vivo and provide for better pharmacological kinetic properties and more effective treatment of AMD in humans [109]. After all these development efforts by researchers at Nexstar Pharmaceuticals, optimally-stabilized and formulated aptamers were marketed as intravenous or subcutaneous injectables with biological half-lives of up to 12 hours $[110,111]$. According to a recent review 8 other aptamers are currently undergoing clinical evaluation for various haematology, oncology, ocular and inflammatory indications [7].

\section{Multifunctional Aptamers}

In practice, aptamers are usually selected one at a time as monomers against a single target. As explained in the previous sections, they are also functionalized as monomeric units. However, they may even become more valuable biotechnological tools if the same- or different types of aptamers can be combined in the same molecule. Multifunctional aptamers have been generated to increase potency, to carry out multiple tasks or for allosteric regulation with potential applications in catalysis, therapy and biosensors.

Besides being linked to ribozymes to regulate catalytic activity, molecules consisting of multiple single-type or multi-type aptamers can have improved potency, activity, functionality and modality. Bifunctional aptamers can be obtained from the modified in vitro selection method called 'chimeric SELEX'. Applying this protocol, the pools of 
chloramphenicol and coenzyme-A (CoA) aptamers were linked together at two ends through a homologous sequence in the middle. This linked pool was then reselected for its ability to bind both chloramphenicol and CoA. After five rounds of selection, bifunctional aptamers were generated with higher affinities for both ligands than the original linked aptamer pool. The enriched bifunctional aptamers bound to both CoA and chloramphenicol with as good affinities as the original CoA and chloramphenicol individual aptamers [112].

Rational design has also been used to generate multifunctional aptamers. For example, a thrombin aptamer, an L-selectin aptamer and a DNA element conjugated to biotin or fluorescein were linked together through duplex linkers or three way junctions. The resulting multivalent aptamers had multiple functions including anticoagulation activity and application as biosensors [50, 113]. Another bifunctional aptamer was obtained by linking the AMP and cocaine aptamers was and then hydridized to a complementary length of DNA. Release of the hybridized sequence by either AMP or cocaine was detected by colorimetric or electrical signals [114].

Once generated, multiple units of aptamers have also been used to knock down several protein functions more efficiently. Twelve units of the B52 aptamer was expressed in cells and in Drosophila melanogaster that showed a 10-fold increase in affinity compared to the monovalent aptamer. Also, its stable expression inhibited B52 functions and reversed the Drosophila phenotypes caused by overexpression of B52 [28]. In addition, an aptamer was selected for CTLA-4, the T-cell receptor with a negative signal for T-cell activation that exists as a dimer cross-linked by a pair of B7-1 proteins. A tetramer of the CTLA-4 aptamer was found to better inhibit CTLA-4 function than its monovalent form and thus enhance Tcell immunity against tumors [115]. Similarly, a multi-thrombin aptamer showed stronger anticoagulant activity than its monomer [113]. Finally, a divalent aptamer against 4-1BB, a 
cell surface receptor on $\mathrm{CD}^{+} \mathrm{T}$ cells, stimulate $\mathrm{CD}^{+} \mathrm{T}$-cell activation whereas the monovalent 4-1BB aptamer was shown to be inactive [116].

Simply, the potency of monomeric aptamers can be increased due to multiaptamers ability to capture more ligands. In 1998, it was reported that both one and three units of tobramycin and kanamycin aptamers, once expressed in E. coli, resulted in drug-resistance which was better with the three of tandem aptamers. In addition, when inserted into the 5'UTR, multi-tobramycin and -kanamycin aptamers inhibited translation in the presence of tobramycin and kanamycin-A [27].

Another purpose of constructing dimer aptamers is to increase the signal and the resolution in imaging. A divalent human neutrophil elastase (hNE) aptamer showed about 20 fold increase in affinity and higher fluorescence compared to its monovalent form [117]. A molecule with multivalent malachite green aptamers was also found to bind more malachite green per molecule and emit a stronger fluorescence than a single malachite green aptamer [118]. Another example is the multivalent MS2 coat protein aptamers which were inserted into the 3' end of lacZ mRNA. They could interact with the coexpressed MS2GFP fusion proteins thereby lacZ mRNA movement imaged inside a cell [119]. However, the results showed that the sensitivity was such that individual lacZ mRNA movement could be observed with 24 linked MS2 aptamers as part of the mRNA, whereas only the movement of multiple lacZ mRNAs could be observed with 6 and 12 units of MS2 aptamers fused with the mRNA [119].

\section{Functional Intracellular Aptamers}

The ability to construct expression systems with aptamers to function inside a cell gives a lot of advantages to aptamers over antibodies and RNA-based therapy. For example, this advantageous property has eliminated the problems of low transfection 
efficiency, and the lysosomal and nuclear localization of the RNAs used in therapies. Therefore, a variety of expression systems that involve aptamer sequences integrated either by themselves or as decoys has been developed. These engineered vectors make the use

of aptamers 1) to control transcription and translation, 2) to inhibit HIV-1 infection, 3) to induce cell death and 4) to give aminoglycoside resistance to the bacteria. In our studies, we have utilized some of these RNA aptamers and their secondary structures obtained via RNAStructure4.6 are shown in Figure 1.

\section{Aptamers to Control Transcription and Translation}

In nature, there have been identified natural riboswitches located in the 5' or 3' UTR of mRNA transcripts to regulate transcription or translation processes by small metabolites [120]. By mimicking the functions of these riboswitches, in vitro selected aptamers can regulate transcription or translation once inserted into the $5^{\prime}$ or $3^{\prime}$ UTR of the desired genes. In fact, the first-reported intracellular application of aptamers was intended to control gene expression via small molecules. This was achieved by inserting three copies of tobramycin or kanamycin-A aptamers into the $5^{\prime}$ UTR of reporter gene, the expression of which was regulated by the aptamer's target. In the presence of $60 \mu \mathrm{M}$ target molecule, more than $90 \%$ of reporter expression was inhibited compared to the controls [27]. In the same report, three copies of an aminoglycoside aptamer was replaced by three copies of an aptamer selected to bind two related Hoechst dyes, $\mathrm{H} 33258$ and $\mathrm{H} 333342$, with which ligand dependent translational regulation was observed in both wheat germ extracts and in Chinese hamster ovary $(\mathrm{CHO})$ cells transfected with DNA encoding the lacZ gene [27]. These first examples of intracellular applications of aptamers encourage other researchers to utilize a variety of aptamers inside cells. 
The malachite green (MG) RNA aptamer has been used to control translation of cyclin. The aptamer, selected for use in RNA chromophore-assisted laser inactivation [121], was inserted right before the start codon (AUG) of a cyclin transcript in Saccharomyces cerevisiae (S. cerevisiae). By this way, translation of a modified cyclin transcript was regulated by a structural analog of MG, tetramethylrosamine (TMR) [122]. They observed that in the presence of TMR, the MG RNA aptamer-ligand complex in the 5'UTR of cyclin transcripts was stable enough to inhibit translation of cyclin by more than $90 \%$. In another example, the MG RNA aptamer was engineered through in vivo (in S. cerevisiae) selection as a TMR-dependent transcription activator [123]. In this study, transcriptional activity of the engineered RNA molecule increased about 10 fold in the presence of TMR.

In a more recent article, researchers described a different route to express aptamers in yeast to inhibit a transcription factor called heat shock factor 1 (HSF1) [124]. They utilized the intron homing process that is induced by Pol I transcription. In this way, they effectively integrated self-splicing introns containing the aptamer sequences into hundreds of rRNA genes. An aptamer against HSF1, a conserved transcription factor responsible for mobilizing specific genomic expression programs in response to elevated temperature, was expressed. As a result, a temperature sensitive growth defective phenotype and decreased level of heat shock gene expression were observed. In addition, they showed that the aptamer can bind yeast HSF1 and its mammalian ortholog with equal affinity, They claimed that successful inhibition would also be possible in mammals because HSF1 enables and promotes malignant growth and metastasis in mammals[124].

\section{Aptamers to Inhibit Viral Infections}

Defeating viruses has been a challenge for decades, especially, after the emergence of human immunodeficiency virus (HIV). Although there have been anti-HIV drugs for 
decades, both prevention and cure remain elusive due to severe side effects and resistant mutants of the virus. However, to control HIV infections in vitro selected aptamers against viral proteins have been developed as chemical antivirals. Aptamers have been selected against HIV-1 reverse transcriptase (RT) [104], HIV-1 Rev [102] and HIV-1 integrase [103] as mentioned earlier. These aptamers were shown to inhibit viral replication in several studies.

Among all, the most obvious target was the HIV-1 RT to develop a way for HIV therapy. In fact, the RNA aptamers selected for HIV-1 RT were shown to be functional to inhibit RT's activity in Escherichia coli (E. coli). To validate this, researchers have utilized the requirement of a base pairing interaction between the Shine-Dalgarno (SD) sequence and $16 \mathrm{~S}$ rRNA in ribosomes for bacterial translational initiation. The coding sequence for the RT RNA aptamer was cloned into a position adjacent to the SD sequence of the lacZ gene in E. coli to target HIV RT protein expressed in E. coli. As a result, in the presence of the HIV RT, the aptamer bound to the expressed RT protein and blocked the access of $30 \mathrm{~S}$ ribosomal subunit to the SD sequence of lacZ gene by steric hindrance. Whereas, without the HIV RT, the expression of lacZ was not affected this proves the successful effect of the aptamers. [125-127]

Aptamers can also be used intracellularly as decoys to interact with the diseaserelated RNA-binding proteins. For example, RT aptamers were used to inhibit type I human immunodeficiency virus (HIV-1) infection as aptamer decoys. An oligonucleotide encoding a 33 nucleotide pseudoknot RNA aptamer targeting HIV-1 RT was cloned into a retroviral vector between mutant tRNA gene and an RNA polymerase III termination signal [128]. The tRNA-aptamer transcripts generated by the RNA polymerase III were found in the cytoplasm after being transiently expressed in several human cell lines. In the human 293T cell line, co-transfected with a pro-viral HIV-1 DNA, inhibition of HIV particle release was reduced by 
more than $75 \%$. Then, the viral particles derived from 293T cells were used to infect human T-lymphoid C8166 cells expressing chimeric tRNA-aptamer and successfully inhibited the subsequent viral production again by more than $75 \%$ compared to the controls [128].

In a similar study, aptamers against HIV-1 reverse transcriptase were combined with small hairpin RNAs (shRNAs) to inhibit HIV replication as in the form of alternative aptamer decoys. The shRNA and the aptamer were inserted downstream of a U6 promoter, utilized by RNA polymerase III, in the MMP-eGFP retroviral vector for blocking endogenous HIV-1 replication [129]. In addition, combining the aptamer with a self cleaving ribozyme at both $5^{\prime}$ and $3^{\prime}$ ends made it possible to produce the exact sequence of the desired aptamer after its intracellular transcription [129]. CEMX 174 cells transfected with the retroviral vector containing aptamer decoy were challenged by the HIV. The results showed that the aptamer decreased the levels of the early RT products up to $96 \%$ at 2 hours post infection, whereas the shRNA-aptamer decoy had as much as $62 \%$ inhibition of the same early RT

products. Even, aptamers were able to inhibit two consecutive rounds of reverse transcription of HIV-1 genome by their ability to get encapsidated into the virion particles. Finally, in the same study, they found that about $85 \%$ of aptamer RNAs and shRNAs were present in the cytoplasm with an RNase protection assay [129].

\section{Aptamers to Induce Cell Death}

RNA aptamers can be used to target endogenous proteins to induce cell death as well. In 2006, five RNA aptamers targeting the Bcl-2 homolog CED-9 were selected and two of these RNA aptamers were used to induce cell death in Caenorhabditis elegans ( $C$. elegans) [130]. Over-expression of either of the two aptamers in six touch receptor neurons in C. elegans induced efficient apoptosis of these neurons. This clearly exemplifies that 
RNA aptamers can be used to modulate programmed cell death in vivo and can potentially be used to develop antagonist drugs to treat human diseases caused by abnormal apoptosis.

Furthermore, aptamers targeting nucleophosmin (NPM), which is a multifunctional protein involved in both proliferation and apoptosis, were used in vivo [131]. Because NPM negatively regulates p53 and is frequently over-expressed in a wide variety of cancers, targeting NPM with aptamers would cause apoptosis. In fact, expression of the selected RNA aptamers had series of effects one of which was to inhibit oligomerization of NPM. This inhibition results in mislocalization of NPM in the nucleoplasm rather than in the nucleolus. Moreover, p14ARF is mislocalized in the nucleoplasm and p53 is upregulated in cells expressing NPM aptamers. Finally, cancer cells expressing NPM aptamers not only underwent apoptosis on their own, but were found more susceptible to apoptosis induced by DNA-damaging agents as well [131]. All together these examples clearly demonstrated that aptamers can be used as anti-cancer agents.

\section{Aptamers to give Aminoglycoside Resistance to Bacteria}

Antibiotic antibiotics have been used extensively to kill bacteria for decades. They are positively-charged chemicals produced by fungi as secondary metabolites and due to their positive electrostatic potential; they can target RNAs with high affinity. In fact, their primary target is the A-site embedded in 30 S ribosomal small subunit $[132,133]$. Once aminoglycosides bind to the A-site, misreading of genetic information occurs and bacteria eventually die. However, researchers showed that this event can be prevented by expressing RNA aptamers that were selected for aminoglycosides; kanamycin and tobramycin [27]. They cloned one and three copies of kanamycin or tobramycin aptamers and expressed with T7 RNA polymerase promoter. Once induced with IPTG, these RNAs 
captured aminoglycosides and prevented the aminoglycoside binding to ribosomes thereby bacteria could grow in the presence of aminoglycosides. As a result, bacteria were able to grow in the presence of 10 and $20 \mu \mathrm{M}$ kanamycin or tobramycin [27]. These successful implementations have indicated that aptamers can be functionally used inside cells. 


\section{MOLECULAR MODELING OF NUCLEIC ACIDS AND APTAMERS}

Biological macromolecules function by way of their specific tertiary and quaternary structures that highly depend, in a complicated way, on their composition (primary structures). This structure-function relationship has led structural biologists to investigate the molecular structures of many biologically-significant molecules, specifically proteins and nucleic acids. These studies pinpoint how these biomacromolecules are shaped and how small changes in their structures affect their function.

Structural biologists use various experimental techniques to accurately determine the structures of a variety of molecules, including X-ray crystallography, nuclear magnetic resonance (NMR), cryo-electron microscopy (cryo-EM), circular dichroism (CD), small angle X-ray scattering (SAXS), multiangle light scattering, ultra-fast laser spectroscopy and dual polarisation interferometry. These methods involve simultaneous measurements on a population of unique molecules. Most methods can only define an average static state of the molecular structures in the molecular population that usually represents a snapshot of highly dynamic processes. In contrast, molecular dynamics (MD) simulations make it possible to obtain highly accurate physiochemical molecular models that can complement the work of structural biologists and expand our understanding of the dynamics of molecular structure.

In a typical MD experiment, each atom (or point charge with an associated mass) is properly defined where the chemical bonds between neighboring atoms are defined as spring-like interactions. In this system, van der Waals forces are described as LennardJones potential while electrostatic interactions are computed based on Coulomb's law. Equilibrium bond lengths, bond angles, partial charges, force constants and van der Waals parameters are all together described as a force field. Using all these force field parameters, atoms are assigned with velocities, related to the temperature of the system and atomic 
trajectories in space and time, that are integrated based on Newton's laws of motion (F=ma). Then, the molecule is free to vibrate, rotate, and translate into favored conformations in response to intra- and intermolecular forces acting upon it. In this way, MD simulations can inform researchers about the possible behavior of dynamic processes as a function of time with the intrinsic inclusion of temperature effects.

So far, several MD simulation programs have been developed, which are based on energy minimization for the analysis of proteins, nucleic acids and lipids. These MD simulations can be carried out either in vacuum (described as gas-phase simulations), in solution (described as explicit solvent simulations) or in crystal environments to study the behavior of molecules. MD simulations can be applied to understanding the mechanisms of enzyme catalysis, protein folding and stability as well as molecular recognition of proteins, DNA, RNA and membrane complexes.

As a result of recent findings about RNA's ability to control vital cellular events and the growing number of diseases linked to RNA malfunction, RNA manipulation has gained more attention. However, to get control over RNA, it is essential to understand the network of interactions that help an RNA to assume its ensemble of conformations. RNA molecules do not have static structures as are often presented from crystallographic data. To the contrary, RNA is a very flexible molecule that can change its conformation/s in response to cellular signals. Therefore, it can control cellular events like turning specific genes on or off by interacting with other biomolecules [134]. In some cases, an RNA can adopt so many possible conformations under different conditions that it cannot be defined as having a single structure [134]. The details of the network of interactions that help form the complex tertiary and quaternary structures of RNAs, obtained through MD simulations for a variety of RNA molecules, have also uncovered some unexpected characteristics of RNAs as discussed below, 
One of the very first examples of MD simulations of an RNA includes the modeling of solvated yeast tRNA ${ }^{\text {Asp }}$ over 500-ps [135]. This study showed the anticodon loop and the acceptor stem as the most flexible regions of the L-shaped tRNA molecule. 5'-U1 and 3'CCA terminal residues and the apical G34 wobble nucleotide were also found extremely flexible. In addition to the flexibility of the molecule, $\mathrm{Mg}^{+2}$ ions were located in the core of the tRNA that help stabilize the four-base triples [135]. This simulation encouraged the other researchers to study other RNA molecules.

These early simulations of yeast $\mathrm{tRNA} \mathrm{Asp}^{\mathrm{Asp}}$, were followed by another MD simulation study that identified two water-mediated uracil-cytosine (UC) base-pairs that switch between different conformations in an RNA duplex [136]. One of these conformations included a water molecule that behaved as a hydrogen donor and acceptor. In the second conformation, the oxygen of a water molecule was found in close proximity to the two-base donor sites. The latter conformation was also observed in 23S rRNA sarcin-ricin domain where it was located in one of the minor groove surfaces [137]. These MD simulations with an RNA duplex revealed for the first time that the dynamic behavior of the water-mediated UC base-pairs differed from the Watson-Crick and non-canonical guanine-uracil base-pairs [136]. As a result, one further step of complexity has been added to the conformational flexibility of the RNA duplexes, which could be attributed to water-mediated UC base-pairs.

Another important group of RNA elements are loop-loop interaction motifs that play a role in encapsidation of the viral genomes [138]. They form structurally and biochemically significant complexes such as in the retroviral RNA genome dimers of HIV-1 and Moloney murine leukemia virus [138]. MD simulations of RNAs with kissing-loop motifs helped understand their structural dynamics and formation of cation-binding pockets [139]. Relaxed intermolecular G-C base pairs and a cation binding pocket formed by the core region of the kissing complexes were first noticed in these MD simulations. In addition, the binding 
pocket was observed as a very flexible region that could effect fluctuations in the asymmetrical bending of the whole molecule. However, different types of kissing loops behaved differently in terms of their cation binding. For example, two hexa-coordinated magnesium ions and one sodium ion were critical for the stabilization of subtype $A$ whereas the pocket of a subtype B kissing loop contains three delocalized sodium ions [139]. With the help of MD simulations, the kissing complexes were found to be more flexible entities than canonical A-form RNA duplexes.

Kink-turns (K-turns) were examined in another study. Some of these K-turns were previously shown to contain flexible elements that could mediate large-scale ribosomal motions [140]. For example, three of these K-turns exhibited hinge-like motions in ribosomal RNA that play critical roles in translation [140]. Solution biochemical analysis indicated that X-ray geometries of K-turns were rather unstable; although, from their X-ray geometries it was not expected that these K-turns would be very flexibility. In MD simulations, K-turns motions were observed as flexible loops controlling two rigid helical stems in hinge-like motions. In sum, this study along with X-ray and in solution biochemical studies has helped a detailed understanding of the K-turns, revealing how molecular interactions can modulate the shape and flexibility of molecules [140].

Discovery of the ribozymes led to the RNA world hypothesis late in the $20^{\text {th }}$ century [141]. RNA molecules were proposed to be one evolutionary step prior to protein-based enzymes in catalyzing chemical reactions. Conformational rearrangements were found to be involved in catalysis by the hepatitis delta virus (HDV) ribozyme. This self-cleaving RNA enzyme that plays a role in the replication of the human HDV was simulated by MD [142]. Crystallographic studies had shown that cytidine at 75 position (C75) was situated in close proximity to the cleavage site both in the reaction precursor and at 3' of the genomic HDV ribozyme. It was confirmed by the MD simulations that site-specific self-cleavage required 
the dynamic binding of $\mathrm{C} 75$ in the catalytic pocket. This binding facilitated the $\mathrm{H}$-bond formation between $U 1$ and C75. Surprisingly a distant RNA tertiary interaction involving a protonated cytidine (C41) was found to stabilize the C75 interaction [142]. Later, from another MD simulation study, the same group showed that a previously unnoticed U-turn motif was utilized by the genomic HDV ribozyme for fast catalysis [143].

Studies of an in vitro selected aptamer led to the discovery of a very interesting phenomenon. The malachite green aptamer (MGA) itself had an unusual property that once it binds to malachite green (MG), it enhances the MG fluorescence [118]. However, in solution, MG alone is rapidly bleached. Experimental studies supported by computational analysis demonstrated that the MGA could inhibit the bleaching of MG by preventing the hydroxylation of MG at the central carbon [144]. The mechanism of action was in a concentration-dependent manner with a stoichiometry, which suggested steric hindrance and exclusion of the hydroxyl ion. This steric exclusion of an hydroxyl ion was demonstrated by computational cavity analysis of the NMR structure as well as being confirmed experimentally [144]. With this study, a nucleic acid aptamer was shown to control access to specific chemical groups on small molecules by steric hindrance.

The structures of RNA molecules share a lot of common features with the anatomy of the human body. As anatomical features of our joints define the range of motion of our arms and legs, RNA junctions is directly affect the motion of RNA helices. A research group from the University of Michigan lead by Hashim Al-Hashimi used molecular dynamics and NMR to investigate the flexibility of transactivation region (TAR) of HIV-1 RNA and the conformational changes that occur upon aminoglycoside binding in this model system [145]. Shown in Figure 4, the chemical structures of aminoglycosides differ from one another in charge, size and other chemical properties. This study revealed that larger aminoglycosides froze RNA in more bent positions whereas smaller ones favored straighter RNA structures. 
Also, further analysis indicated that, when positioned between two helices, the aminoglycoside acts like a wedge, pushing the helices apart [134]. This study along with many others has clearly demonstrated that MD simulations are very helpful to investigate the complex network of interactions in RNA structures as well as to increase our understanding of the structures refined from X-ray and NMR structural data. 


\section{RIBOSWITCHES}

Cells have evolved a variety of complex control mechanisms to efficiently use their energy resources. The degree of complexity of control mechanisms at different levels gives them an opportunity to spatiotemporally regulate cellular events essential for their survival. At the gene expression level, most of the control has previously been believed to be maintained by DNA binding proteins. However, recently it was shown that one type of control mechanism involves metabolite-sensing mRNA units called riboswitches (earlier called "regulons" [146]. They are highly selective and can even discriminate against closely related analogs of their targets. Cells use riboswitches as cis-acting RNA-based sensors to rapidly adapt to their microenvironment. Through the riboswitches cells can link changes in metabolite levels due to changes in flux through metabolic pathways to changes in transcription and translation of relevant enzymes [147, 148]. These metabolite-sensing RNA units are in general located in the non-coding portions of mRNAs where they serve as receptors of specific metabolites to regulate gene expression [149, 150]. For riboswitch regulation, the helical stability, dynamic sampling and conformational selection of the RNA units are important [151]. Their mechanisms of action directly depend on ligand binding that, in turn, results in conformational changes in the three-dimensional structures of the

riboswitches. These structural rearrangements influence RNA binding or translocation by the ribosome, which affects the efficiency of translation initiation [152].

\section{Natural Riboswitches}

Riboswitches have a wide range of targets or effector molecules and can be divided into two groups of natural and engineered riboswitches. Natural riboswitches recognize amino acids like lysine [153] and glycine [154]; amino acid derivatives like Sadenosylmethionine (SAM) [155-157] and S-adenosylhomocysteine (SAH) [158]; 
coenzymes like flavin mononucleotide (FMN) [159], thiamin pyrophosphate (TPP) [159], coenzyme B12 [160]; simple sugars like glucosamine 6-phosphate (Glcn6P) [161]; purines like guanine, cyclic di-GMP, adenine and pre-queuosine (preQ1) [162, 163]; and tRNA [164]. Also, they can sense metal ions like molybdenum cofactor and magnesium ions $[164,165]$. Riboswitches can even sense temperature and $\mathrm{pH}$ that are the determinant physicochemical factors for bacterial survival $[166,167]$.

Naturally occurring riboswitches have very complex three-dimensional structures, which provides them with the ability to achieve a very specific and targeted regulation of gene expression. Their structure is composed of an aptamer portion and an expression platform. The aptamer recognizes its cognate small effector molecule and this interaction leads to global structural changes in the expression platform, which functions as the regulator of gene expression. The size of nucleotide binding aptamer domains ranges from $40 \mathrm{nt}$ to $300 \mathrm{nt}[150]$.

\section{Mechanisms of Riboswitches}

Riboswitches typically turn off gene expression in response to the binding of their effectors, but some turn it on. They can target transcription, translation initiation or mRNA stability. Prokaryotic riboswitches that regulate transcription by an "off" mechanism promote the formation of rho-independent transcription termination hairpins, which consist of a GCrich stem followed by a poly-U tail and leads to premature transcriptional termination. The structural rearrangement that occurs as a result of the riboswitch binding its effector results in the arrest of transcriptional elongation due to the destabilization of the RNA-RNA polymerase complex. Inhibition of translational initiation by prokaryotic riboswitches is achieved by their folding so as to sequester ribosome-binding sites like the Shine-Dalgarno box or the start codon (AUG). Finally, the stability of the mRNA is affected by a ribozyme in 
the $g / m S$ riboswitch. The riboswitch cleaves itself when it binds glucose 6-phosphate and this interaction leads to rapid mRNA degradation [168].

Another type of regulation by riboswitches involves alternative splicing. For example, a TPP riboswitch in plants modifies splicing and alternative $3^{\prime}$-end processing $[169,170]$. In Neurospora crassa, a TPP riboswitch produces an upstream Open Reading Frame (uORF), which in turn affects the expression of downstream genes [171].

A very rare example of regulation of gene expression by riboswitches involves an RNA element, such as the Gcv element, that activates gene expression. Glycine binding to the $g c v$ element promotes a structural rearrangement of the riboswitch that involves formation of an anti-terminator structure, which competes with the terminator helix in mRNA. The structural change shifts the transcription into an active state [172].

\section{Specific Examples of Riboswitches}

Riboswitches control genes that encode proteins involved either in their ligand synthesis pathway or as transporters of their ligands. In-line probing, which was developed to detect conformational changes in mRNA, was used to identify the riboswitch in btuB gene that is regulated by coenzyme B12 (AdoCbl) [160]. The genes controlled by the cobalamin riboswitch include a membrane protein for the transportation of coenzyme B12 and essential genes for its metabolites. The following examples summarize the types of riboswitches identified so far.

The RFN-element or FMN riboswitch recognizes flavin mononucleotide (FMN) and regulates riboflavin biosynthesis and transport $[159,173]$. The THI-box or alternatively TPP riboswitch found in the 3' UTR of plant genes bind thiamin pyrophosphate (TPP) to regulate thiamin biosynthesis and transport, as well as transport of similar metabolites. It is one of the few riboswitches found so far in eukaryotes [159, 174]. 
Two types of amino acid binding riboswitches have been identified: 1) the glycine riboswitch that binds glycine and regulates genes in glycine metabolism, including the use of glycine as an energy source, and 2) the lysine riboswitch (or L-box) binds lysine to regulate lysine biosynthesis, catabolism and transport [153]. So far, the glycine riboswitch is the only known natural RNA with cooperative binding to its effector, which is accomplished by two adjacent aptamer domains located in the same mRNA [172].

Amino acid derivative-binding riboswitches are the SAH and SAM types. SAH riboswitches bind S-adenosylhomocysteine to regulate genes involved in recycling the SAH metabolite that is produced when used in methylation reactions. To regulate methionine and S-adenosylmethionine (SAM) biosynthesis and transport, three distinct SAM riboswitches have been identified as SAM-I (originally called S-box) [157], SAM-II [175] and the $S_{M K}$ box [176]. Although they all switch off gene expression in response to SAM binding, no sequence or structural similarities have been observed between these riboswitches. They differ in structural arrangements of their aptamer regions, phylogenetic distributions and mechanisms of regulation. SAM-I riboswitches typically function through transcription terminator formation whereas SAM-II riboswitches inhibit translation via ligand-dependent sequestration of the ribosome-binding sequence.

Purine riboswitches regulate purine metabolism and transport. The specificity for either guanine or adenine depends completely upon Watson-Crick interactions with a single pyrimidine in the riboswitch at position $\mathrm{Y} 74$ [172]. In the guanine riboswitch this residue is always a cytosine (i.e. C74), in the adenine residue it is always a uracil (i.e. U74). Deoxyguanosine is recognized by homologous purine riboswitches that are more significantly different from each other than a single nucleotide mutation observed the guanine riboswitches. Also, preQ1 riboswitches bind pre-queuosine and regulate genes involved in the synthesis or transport of this precursor to queuosine. Two entirely distinct 
classes of PreQ1 riboswitches are known: PreQ1-I riboswitches and PreQ1-II riboswitches. The binding domain of PreQ1-I riboswitches is unusually small among naturally occurring riboswitches. PreQ1-II riboswitches, which are only found in certain species in the genera Streptococcus and Lactococcus, have a completely different structure, and are larger than pre-Q1-1 riboswitches $[162,163]$. Two classes of cyclic di-GMP riboswitches are also known: cyclic di-GMP-I riboswitches and cyclic di-GMP-Il riboswitches. They bind the signaling molecule cyclic di-GMP in order to regulate a variety of genes controlled by this second messenger [163].

Finally, the T-box RNA is interacts with both charged and uncharged tRNAs, by which it monitors the substrate to product ratio rather than the absolute amount of its substrate. In this system, binding of uncharged tRNA is competitively inhibited by aminoacylated tRNA [177].

\section{Engineered Riboswitches}

The power of controlling gene expression patterns has motivated researchers to discover new riboswitches that could be applied in therapy. We can divide these into three types based on their content. The first type consists of self-cleaving ribozymes, the second utilizes cis or trans-expressed antisense RNAs and the final one has only the aptamer sequence that can respond to an external effector.

Several riboswitches have been engineered using ribozymes that can be regulated by an external effector $[178,179]$. In these studies, self-cleaving ribozymes were utilized to control gene expression in mammalian cells. The regulation was obtained by inhibiting ribozyme activity through the external addition of toyocamycin or 5-fluorouridine (5FU). The authors utilized the luciferase gene as a reporter and detected $\sim 365$-fold and 110 -fold enhancement in luciferase expression with respective effectors [179]. 
In 2004, Isaacs et al. demonstrated both trans and cis repression of gene expression in prokaryotic cells using an engineered riboswitch [180]. They placed an internally complementary sequence directly upstream of the ribosome-binding site that forms a stemloop structure, which interferes with ribosome binding. Also, they targeted this stem loop by expressing a small non-coding RNA in trans that reversed the effect of the riboswitch and increased gene expression [180]. In a similar vein, anti-switches were developed as translational ribo-regulators in the form of trans-acting RNAs that could respond to small molecules by creating hybrid riboswitches. In this system, the binding of theopylline to its cognate aptamer was able to open up the structure of a sequestered sequence for an antisense to bind and thereby prevent GFP expression [181].

The third class of synthetic riboswitch solely depends on small molecule binding aptamers that are inserted into the 5' UTR of an mRNA [27, 182, 183]. The small molecule binding to these RNA aptamers alters their structures to affect translation of the downstream coding regions. In this context, either the binding of the eukaryotic ribosomal subunit to the cap-structure or its scanning along the mRNA is interfered with by the aptamer-ligand complex [184]. So far, only a few small molecule-binding aptamers have been described as functional riboswitches $[27,122,182,183,185]$. These aptamers are targeted by theophylline, tetracycline, neomycin-B, or Hoechst 33342. For these riboswitches the presence of the cognate ligand results in structural rearrangement of the aptamer to prevent translation. A neomycin-B binding riboswitch, controlled by extracellular neomycin-B, was selected with the aid of a GFP reporter system [183]. Other RNAs known to bind neomycinB were also tested with the GFP reporter construct, including the naturally occurring $16 \mathrm{~S}$ rRNA A-site region [186] and other previously selected neomycin-B aptamers [187]. However, only the neomycin aptamer specifically selected with riboswitch functionality was 
found responsive to neomycin-B. This result suggests that the ability to behave as a riboswitch requires structural features beyond those that result in tight effector binding.

\section{Future Perspectives}

The role of RNA elements as riboswitches in prokaryotic gene regulation was not widely recognized until the beginning of the 21 st century. As of 2012 , there are currently about 700 articles in the PubMed database for the term "riboswitch". In addition to these, there is an extensive literature for the RNAs playing a role in regulation of bacterial gene expression that predates the use of the term. Also, recent findings that have identified the first few riboswitch elements in eukaryotic systems imply that these RNA-based regulatory mechanisms are used even more widely than is currently known.

The ability to modulate gene expression with an extracellular binding partner would enable researchers to control the level of a particular gene with riboswitches. These newly developed biotechnological tools then can be utilized in therapy as gene expression control units. Bacteria develop drug resistance through many alternative routes, one of which is to use riboswitches to control expression of certain genes targeted by the drugs. In addition, the emergence of drug-resistant organisms that utilize riboswitches suggests that it is possible to discover novel drugs and new therapeutics by targeting these riboswitches [188].

The growing dataset of genomic sequences and bioinformatics techniques provide the knowledge that is necessary for developing tools for the identification of riboswitches. However, we still need to understand the relationship between ligand-bound and unbound states of riboswitches in order to fully understand their mechanistic and structural features and their physiological roles in the cell. The ability to predict the means by which a particular RNA aptamer can be modified to provide it with riboswitch activity will allow for a rapid increase in the number of RNA switches available for application in synthetic biology. 
Understanding riboswitch function from a structural basis will also speed the development of new antimicrobial drugs that can target riboswitches. These results will be the outcome of multi-disciplinary studies that are likely to be an area of active research for the next generation of researchers. 


\section{IMAGING TECHNOLOGIES AND RNA DETECTION}

Molecular imaging covers noninvasive as well as invasive methods used to investigate the molecular events inside organisms. The ability to image molecular events brings a number of exciting possibilities for medical applications from basic pharmaceutical developments to early detection and treatment of diseases. Potential reporter systems have been explored using various imaging modalities including radioactivity, bioluminescence and fluorescence [189].

Radiotracer methods have been widely used for imaging living subjects noninvasively. Two of these methods are single photon emission computed tomography (SPECT) and positron emission tomography (PET) which are used to detect isotopes emitting gamma rays and positrons respectively. Each of these methods has its own advantages and disadvantages. For example, gamma $(\mathrm{\gamma})$ rays, penetrating through tissues, can be detected directly by gamma cameras, which can produce three dimensional tomographic images when rotated around the subject for SPECT [190]. In contrast, positrons emitted in PET cannot penetrate through a tissue, but instead collide with electrons and undergo annihilation, which causes two gamma rays to be emitted at close to 180 degrees that are detected for imaging. Although different isotopes cannot be distinguished in PET because the gamma rays created from positron annihilations have the same energy (511 keV), PET offers over ten times the sensitivity of SPECT. Gamma rays emitted directly from atomic nuclei can have a range of energies; thus, dual-isotope SPECT can be used to image several isotopes simultaneously provided that the energies of their gamma rays are sufficiently different [191]. One of the commonly used SPECT tracers in cancer biology is ${ }^{18} \mathrm{~F}$-deoxyglucose (FDG), which is immediately modified to FDG-phosphate 
by the high level of expression of hexokinase in cancer cells [192]. By this means growing tumors can easily be detected by FGD in vivo.

Gene expression levels change in many diseases and at different developmental stages. The development of noninvasive means of detecting changes in gene expression levels is the critical step for monitoring progression of diseases and normal developmental events in order to understand them more fully and for effective diagnosis and treatment, in the case of disease. So far, several gene reporter systems have been developed to determine the level of gene expression as well as the location of the reporter. A vector can be engineered with the promoter placed upstream of the reporter gene to control its expression. Then, the reporter gene products can be detected and quantified from which the transcriptional activity of the promoter can be estimated.

\section{Reporter Genes in Imaging}

Early reporter genes include mainly enzymes like chloramphenicol acetyltransferase (CAT), thymidine kinase, alkaline phosphatase, luciferase and $\beta$-galactosidase [193]. Luciferase and $\beta$-galactosidase can also be analyzed in situ, to identify specific cells that express these enzymes [194]. These two assays require the external addition of the ligands, luciferin and o-nitrophenyl- $\beta$-galactoside (ONPG). Luciferase from the firefly (Photinus pyralis) catalyzes the ATP driven oxidative decarboxylation of luciferin, resulting in the emission of a photon of light that can be detected with a luminometer. It has gained popularity because the luciferase assay is simple and is up to 1000 -fold higher more sensitive than the CAT assay [194, 195]; its bioluminescence is sufficient to detect the enzyme in fewer than a hundred cells. The availability of the luciferase and $\beta$-galactosidase genes has simplified the efforts to quantify the activity of transcriptional regulatory elements. 
Also, the efficiency of gene delivery methods like transient transfection can be determined with the use of these reporter genes.

The improvements in the detection devices increased the sensitivity of the measurements. For example, limited penetration of light through tissues was a challenge for detection of excitation and emission. However, detection of bioluminescence in vivo is significantly improved by the use of charge coupled device (CCD) cameras that are cooled to $-120^{\circ} \mathrm{C}$. With increased sensitivity and signal-to-noise ratio of CCD cameras, it is possible to detect luciferase activity in deeper tissues [196]. Although bioluminescent imaging does not provide high resolution, with the discovery of fluorescent proteins and advancements in fluorescent microscopy make it accessible to image fluorescence at single cell resolution within tissues [197].

In 1962, green fluorescent protein (GFP) was discovered while isolating the bioluminescent protein aequorin from the jellyfish Aequoria victoria [198]. Aequorin emits blue light $(470 \mathrm{~nm})$, while the intact organism emits green light $(509 \mathrm{~nm})$. It was demonstrated that the luminescence from aequorin is transferred to GFP, which then emits green light. GFP has a maximal excitation at $400 \mathrm{~nm}$ with a second small peak at $480 \mathrm{~nm}$ and it has emission maximum at $508 \mathrm{~nm}$ with the quantum yield of 0.72 when excited at 470 nm [199]. GFP has a spontaneously formed chromophore core (phydroxybenzylideneimidazolidinone) that is created by cyclization and dehydrogenation among the three amino acids Ser65-Tyr66-Gly67 [200, 201]. In addition, expression of GFP in E. coli and C. elegans displayed identical fluorescence properties to natively expressed GFP in A. victoria [202]. Since these earlier successful studies, fluorescent proteins have been used extensively as reporters of gene activity.

Modifications in GFP were made of certain properties like the efficiency of folding and chromophore formation, endogenous expression levels, fluorescence intensity and the 
excitation and emission wavelengths. GFP variants were engineered by inducing random mutations of the 20 amino acids in and around its chromophore core. One mutant (GFPmutl; F64L, S65T) exhibited a 35-fold increase in fluorescence and improved folding, which also results in about 100-fold increase in fluorescence in $E$. coli 1 hour after induction of its expression [203]. Based on this mutant, enhanced green fluorescent protein (eGFP) was developed by Clontech introducing silent mutations with preferred human codons which resulted in a 4-fold increase in expression of eGFP in human cells compared to GFPmutl $[204,205]$.

Similar mutational and screening experiments have been used to develop different color variants of GFP such as cyan, blue and yellow fluorescent proteins (eCFP, BFP and YFP, respectively) $[206,207]$. Even variants with increased brightness, low sensitivity to $\mathrm{pH}$ and chloride ions and resistance to photobleaching were developed [208-211]. Also, DsRed, a tetrameric red fluorescent protein from Discosoma striata was discovered [212]. Modifications gave rise to a variant of DsRed with less than a 1 hour maturation half-time [213] and another one with monomer called mRFP1 [214]. Then, mutations of the mRFP1 were made to create a number of fluorescent proteins in the orange to far red spectrum, notably mOrange, tdTomato, mCherry [215] and mPlum [216].

As fluorescent proteins do not require a substrate like reporter genes do, imaging intracellular cell components and the localization, movements and interactions of desired proteins can be elucidated by generating fluorescent fusion proteins directly in living cells by fluorescent microscopy. In addition, changes in gene expression in specific cells can be estimated indirectly. Even, the study of several simultaneous processes can be achieved by tagging the desired proteins with differently-colored fluorescent proteins. Especially, with certain pairs of fluorescent proteins like green and yellow fluorescent proteins, one can study interactions between different proteins if they are in close proximity by Förster 
resonance energy transfer (FRET), which occurs over distances of less than $10 \mathrm{~nm}$ [217, 218]. Recently, bimodal reporter systems with both bioluminescence and fluorescence were used to study human neural stem cells (hNSCs) and glioma cells implanted into the brains of mice. This system provided the possibility for both real time imaging of global cell survival and migration using bioluminescence, as well as determining the fates of implanted cells at single cell resolution using fluorescent proteins [219].

In summary, reporter genes have many innovative approaches used for real-time imaging. However, reporter gene technology utilizes the expression of proteins that needs a lot of energy. Protein synthesis is a high energy-requiring event common in all cell types. In addition to the amount of energy required for amino acid synthesis, transport and activation of proteins contribute to this high energetic cost. An estimate of 7.52 ATP energy equivalents was calculated from whole body measurements for the synthesis of protein from already formed amino acids [220]. Considering the size of commonly used reporter proteins in imaging as an average of $50 \mathrm{kDa}$ protein, the energy needed for its synthesis can be calculated as $375 \mathrm{~K}$ ATP molecules. In addition, the studies with reporter gene systems generally analyze cell lines in culture, where they are provided with high concentrations of

amino acids and other nutritional requirements in the medium. Under these conditions, very few effects of expressed reporter proteins have been reported for cellular behavior and differentiation.

\section{RNA Detection}

Although protein-based imaging technologies have been extensively developed, the available methods for detecting and imaging RNA are significantly limited. First of all, most of the available methods are not really suitable for imaging RNA in living cells because they either require the purification of RNA samples from cells and/or tissues or the fixation of cells. 
However, irrespective of the method that has been used, the results from these detection methods have provided crucial information about the level of RNA expressed inside cells. In this RNA detection section, Northern blotting, fluorescent in situ hybridization (FISH), fluorescence in vivo hybridization (FIVH), stem-looped probes designed for RNA detection, in vivo RNA labeling with bromouridine 5'-triphosphate (Br-UTP) and RNA detection with fluorescently-tagged proteins are discussed.

One of the earliest methods used to detect the level of gene expression is northern blotting where its isolation, RNA is separated by electrophoresis, transferred onto a membrane then hybridized with labeled nucleic acid probes while associated with the membrane[221]. The detected signal on the membrane coming from the labeled-probe hybridized to the target is proportional to the amount of the RNA present in the sample.

Unlike in northern blotting, which requires purification of RNA, nucleic acids can also be detected and imaged in fixed cells using fluorescent in situ hybridization (FISH) which utilizes fluorescently-labeled oligonucleotide probes that are complementary to the target RNA or DNA sequence. These probes are applied to fixed cells followed by the removal of unbound probes by washing [222]. FISH has been used mainly in applications where the target is DNA, such as localizing a specific sequence on chromosomes [223]. However, FISH was also used to visualize single mRNA transcripts [224] and the cellular distribution of mRNA [225]. Although FISH is very useful for identification and localization of specific mRNA species, this technique requires that the cells or tissues to be studied are first fixed.

Fluorescence in vivo hybridization (FIVH) was developed as a method of using fluorescent labeled unstructured linear nucleic acid probes to detect and image specific RNAs in the living cells [226-228]. Unlike in FISH experiments, the washing step to remove excessive probes cannot be done in FIVH. Therefore, to differentiate the true signal from background caused by unbound probes in the cell, dual probes for a specific RNAs were 
designed in which one probe has a fluorescence molecule attached to the 3 ' end to act as a donor and the second probe attached to the $5^{\prime}$ end to act as an acceptor in the FRET pair [228]. Two probes were designed to target adjacent regions on the selected RNA molecule; so that, a FRET signal was only produced when both probes interacted with the RNA target. Although the dual probe FIVH was considered as an improvement in reducing the high background in FIVH, it is not feasible as a method due to the unstructured linear probes that limit its application in cell culture. For instance, detecting single nucleotide polymorphisms (SNPs) is not possible with linear probes in the temperature range in which cells can survive. In addition, micro-injection of the probes is necessary in order to have a high enough concentration of the chemically modified probes in the cells because the probes cannot be produced by the cells $[229,230]$.

To eliminate the disadvantages of the linear probes used in $\mathrm{FIVH}$, the stem-loop structured probes have been developed. Stem-loop structured hybridization probe is a single-stranded oligonucleotide and consists of a sequence complementary to the target and harbors a fluorophore-quencher pair at the 5'- and 3' ends. They can go through a conformational change upon binding to the target and release the fluorescence signal that was quenched due to the presence of the quencher. These structurally constrained probes were shown to have significantly higher specificity than unstructured linear probes by thermodynamic analysis of stem-loop probes with matched and mismatched targets [231, 232]. This can be explained by the smaller number of configurations with lower free energies for annealing with the target [233]. Besides, these probes are highly stable, and stems of 5 to 6 nucleotide seem to offer the best option for its efficient hybridization to the target RNA and for high signal to noise ratio [234]. Because of these advantages of stemloop structured probes over linear unstructured probes, it is reasonable to use stem-loop structured probes in the detection and imaging of specific RNA in living cells. 
One stem-loop structured probe that has also been used in real-time RNA imaging is the 'molecular beacon' [235], single stranded oligonucleotides containing a 15-30 nucleotide loop region closed with 5 to 6 base pairs long stem. A quencher and a fluorophore are attached to ends so when the molecular beacons form stem-loop structures in the absence of target RNA, the fluorescence is quenched. Hybridization with the target nucleic acid opens the stem and the fluorescent signal from the fluorophore is freed. For detecting the mRNA, which is located in the cytoplasm, the location of nucleic acid probe is important. However, because they are chemically modified, the molecular beacons must be microinjected and it was found that once microinjected oligonucleotides were rapidly transported into the nucleus from the cytoplasm [236, 237]. Conjugating a peptide to molecular beacons that can pass through membranes made an efficient delivery and prevented the molecular beacons from accumulating in the nucleus. Tat-peptide-conjugated molecular beacons were used to detect human GAPDH and survivin mRNA in living cells using fluorescence [238]. Nuclease-resistant molecular beacons were conjugated with streptavidin and used to target native p-actin mRNA in living cells and motile chicken embryonic fibroblasts [239, 240].

Fluorescent labeling of nascent RNA was achieved by incorporating 5-bromouridine 5'-triphosphate (Br-UTP) into RNA transcripts by RNA polymerase II (RPII) followed by fixation then detection with anti-BrdU antibodies [241]. The spatial distribution of transcriptionally active chromatin is essential to better understand the functional significance of nuclear compartmentalization. The distribution of sites of active transcription by RPII was detected by labeling nascent RNA with Br-UTP, in vitro and in vivo. Nascent RPII transcripts were found in over 100 defined areas, scattered throughout the nucleoplasm with no preferential localization in either the nuclear interior or the periphery. As a result, they concluded that each of these transcription sites may represent either the activity of a single 
gene or of a cluster of active genes. Also, including antibodies against a splicing factor, SC35, they observed no correlation between intensely labeled SC-35 domains and sites of premRNA synthesis. On the contrary, many sites of RPII synthesis co-localized with weakly stained areas implying that co-transcriptional splicing took place in these weakly stained regions. They conclude that these areas might have also been the sites where splicing was completed post-transcriptionally and the intensely labeled SC-35 domains might have functioned as sites for assembly, storage, or regeneration of splicing components, or as compartments for degradation of introns [241]. Nonetheless, imaging the nascent transcripts in the living cells with Br-UTP label all mRNAs in general and unfortunately can not be applied to specific RNA species.

A method of tracking RNA in living cells was developed by tagging an mRNA with six tandem repeats of a 19-nucleotide RNA stem loop that serve as targets for the RNA binding protein MS2. In that study, MS2-GFP fusion proteins were used to detect the tagged Ash1 mRNA [242]. A nuclear-localization signal was included in MS2-GFP fusion protein which was co-expressed with the MS2-tagged Ash1 mRNA in cells. After Ash1 mRNA was synthesized in the nucleus, its movement and localization could be traced and observed in the living cells by tracking the fluorescence of GFP. Then, it was also confirmed that Ash1 mRNA molecules were also associated with other proteins related to the transportation and localization [119, 243]. Although this method can be used for any RNA of interest, it has disadvantages. First, it requires the co-expression of the MS2-GFP protein and, as explained in the 'reporter genes in imaging' section, expressing proteins is energetically not favorable. Also, same amount of GFP signal is always present and is redistributed upon binding to the tagged RNA, which makes detection very difficult due to the background noise. Additionally, the extra RNA sequence and multiple MS2-GFP fusion proteins add approximately $270 \mathrm{kDa}$ on the nascent RNA, which could have significant effects on the 
movement and function of the RNA [189]. In a similar study, the ability of Exu to bind bicoid mRNA was used to trace bicoid mRNA inside living cells with GFP-Exu fusion protein. Drawbacks of this method were that these constructs could only be used to visualize bicoid mRNA the GFP signal remains in the cells whether or not the RNA exists, thereby decreasing the signal to noise ratio. Although the latter is not a problem as long as the signal to noise is large enough, it remains necessary to distinguish the true signal from background in these systems. Thus, there are still a number of challenges for detecting and quantifying RNA targets in living cells in real time.

More recently, new strategies have been adopted by using split-GFP for the visualization of specific mRNAs in live cells by which it was aimed to eliminate the fluorescence background signal. In the expression systems, two motifs that bind to two different RNA binding proteins are placed next to each other on the target mRNA. Then, the binding of two RNA binding proteins fused with two 'halves' of GFP in proximity on the target RNA mediates the assembly of a functional GFP. By this split-GFP method, researchers have visualized intracellular RNA distribution and dynamics in living cells [244]. However, this method has also a potential limitation, pointed out by in vitro studies, due to the metastability of the reconstituted GFP: once the two halves have assembled into a functional conformation, they do not dissociate, even if the molecule that initially brought them together disappears [245]. As a result, it may be difficult to use this approach to image fast dynamic processes related to the synthesis and degradation of mRNAs. 


\section{DRUG DEVELOPMENT AND RESISTANCE}

\section{Drug Development}

Drug development is a general term used to define the whole process of bringing a new drug with a promising activity against a particular biological target in disease to the market. This process starts with drug discovery and its chemical characterization (makeup, stability, solubility) followed by pre-clinical research, which includes microorganisms and/or animals and then clinical trials on humans. Drug development constitutes a number of tests designed to determine the major toxicities of a newly-developed compound before its use in human. An assessment of major organ toxicity has to be performed to make sure the candidate drug does not have negative effects on the functioning of the heart and lungs, brain, liver, kidney, digestive system, and on other parts of the body. Because the complex interplay of metabolism and drug exposure on toxicity can only be examined in an intact organism, some of the tests need the use of experimental animals while some others can still be performed using in vitro cultured cells. The process of drug development continues after human clinical trials because it is also crucial to ensure that long-term or chronic toxicities of the drug are determined, as well as its effects on other systems like fertility, reproduction and immune system. Then, the compound goes through carcinogenicity testing to determine its risk of causing cancers. Most drugs fail during drug development, either because they have some unacceptable toxicity, or simply because they do not work in clinical trials. Finally, the ones that pass through all these tests can be submitted for marketing approval in the various countries in which they will be sold.

It was estimated in 2002 that approximately $\$ 800$ million was needed to be spent to

bring a new innovative drug to market $[246,247]$. In a more recently published study the cost was reported to vary between around 500 million to 2,000 million dollars depending on 
the therapy or the developing company [248]. This $\$ 800$ million quoted is the cost of all drug development including those that did not result in a new drug. It also includes some $\$ 400$ million of opportunity costs. There are also thousands of other drugs whose development costs are much lower. In sum, all together the pharmaceutical industry spends about half of its research budget on drug development but unfortunately has not made any direct improvements to a physician's ability to treat disease due to the emergence of new drug resistant strains.

\section{Drug Resistance}

Many drugs are developed each year by drug resistance continues to be a big hurdle for treatment of many diseases including cancer, HIV, tuberculosis and many others caused by bacteria, fungi and protozoa. Drug resistance is the result of evolutionary selective pressure, which in turn results in many drug resistant organisms. In clinical practice, drug resistance becomes a critical problem when the drug dosages needed to kill infectious agents are toxic. In this section, only cancer and antibacterial drug resistance are discussed.

Antibacterial resistance is the ability of certain cells and/or microorganisms to withstand attack by antimicrobials. The rise of resistant pathogens threatens lives and wastes limited healthcare resources. According to the World Health Organization (WHO) 2010 report, antimicrobial resistance is mainly due to the overuse and misuse of antimicrobials in human medicine and animal husbandry over the past 70 years and has resulted in death, increased suffering and disability and higher healthcare costs'. Some cancer cells can become resistant to more than one drug. This is observed in tumors where cells develop resistance to the drugs used in chemotherapy [249].

There are four main mechanisms by which drug resistance is acquired [250]. The first is drug inactivation or modification by enzymatic deactivation and/or detoxification. An 
example of this mechanism is the production of $\beta$-lactamases by some penicillin-resistant bacteria [251]. The second mechanism is the alteration of target site/s. A good example for this mechanism is the methylation of $23 \mathrm{~S}$ rRNA resulting in erythromycin resistance. A plasmid-determined methylase dimethylates two specific adenine residues in 23S rRNA of the 50S ribosomal subunit so that binding of the erythromycin-lincomycin type of antibiotics is prevented, thereby making the cell resistant to high levels of these drugs [252]. A third way to get drug resistance is by altering one or more endogenous metabolic pathways. Sulfonamide-resistant bacteria are an example for acquiring drug resistance that results from alterations in the chromosomally encoded dihydropteroate synthase (DHPS) gene, which was linked to at least three other genes of folic acid pathway [253].

Reduced drug accumulation inside the cells is the fourth mechanism for drug resistance. It results in decreasing drug permeability and/or increasing active efflux of the drugs across the cell surface. This is a common way for many cell types like bacteria, fungi and cancerous cells to become drug resistant [254-257]. The main players in reducing drug accumulations are the multidrug resistant pumps, which can be categorized based on their bioenergetics and structural characteristics $[258,259]$. They include the following members in bacteria; 1) the major facilitator superfamily (MFS), 2) the ATP-binding cassette (ABC) superfamily, 3) the small multidrug resistance (SMR) family, 4) the resistance-nodulation-cell division (RND) superfamily and 5 ) the multidrug and toxin extrusion (MATE) protein family [260]. The Acr family members in the RND superfamily play an important role in aminoglycoside resistance through expelling the drugs from cytoplasm [261].

First category: The major facilitator superfamily (MFS) is one of the largest families of transporters and QacA, QacB and QacR were the first examples of this class [262]. They actively pump out monocationic biocides and dyes like benzalkonium chloride, cetyltrimethylammonium bromide and ethidium bromide and QacA additionally can pump 
out dicationic biocides such as chlorhexidine and pentamidine isethionate. The crystal structure of QacR showed that drug-binding pocket is large $\left(1100 \AA^{3}\right)$ and binds a variety of drugs [263]. In the same study, it was found that drug binding relies mostly on stacking and van der Waals interactions but also involves a few hydrogen bonds and the charges of the ligands are usually neutralized by acidic residues in the pocket. Two ligands can simultaneously interact with QacR in different mini-pockets of the binding site [264]. The MFS family is EmrB of $E$. coli, conveys resistance to carbonyl cyanide, mchlorophenylhydrazone and to antibiotics such as nalidixic acid and thiolactomycin [265]. EmrB functions with a periplasmic adaptor protein, EmrA, that connects the pump to an outer membrane channel, TolC, to export the drugs directly into the medium. Another MFS member, NorA produces resistance of $S$. aureus to fluoroquinolones, cationic dyes and cationic inhibitors including puromycin and tetraphenylphosphonium in [265]. Drug efflux makes a strong contribution to resistance through chromosomally encoded MFS pumps, NorA, NorB and NorC, in more than half of $S$. aureus strains isolated from the bloodstream of patients [266].

Second category: The transporters of the ATP-binding cassette (ABC) superfamily have a limited role in drug resistance in bacteria [267] whereas in cancer cells they play crucial roles [268]. For example, LmrA of L. lactis is homologous to $50 \%$ of the mammalian MDR1 proteins [269] but it has no role in drug resistance of L. lactis. Nevertheless, its overproduction in E. coli conferred resistance to the cationic dyes, daunomycin, and triphenylphosphonium. Biochemical studies led to a proposed mechanism in which ATP hydrolysis is coupled with drug extrusion [270]. Furthermore, X-ray crystallographic studies of BmrA of B. subtilis [271], Sav1866 of S. aureus [272] and MacB of E. coli [273] confirmed their potential role in resistance to macrolides when overexpressed. 
Third category: Small multidrug resistance (SMR) family members contribute to the efflux of cationic compounds such as quaternary ammonium biocides or ethidium. In this class, EmrE of $E$. coli is the smallest transport protein known with a single charged residue (Glu14) in the transmembrane domain [274]. This residue has an unusually high pKa of about 8.5 and it becomes deprotonated upon substrate binding. In this way, efflux of the substrate is coupled to influx of proton(s) by a common binding site.

Fourth category: RND-containing pumps of gram-negative bacteria are energized by a proton motive force and can pump out drugs, from the periplasmic space capturing various nonphospholipid, amphiphilic molecules. Structurally, they exist as a tripartite complex containing an outer membrane protein, TolC [275] and the periplasmic protein, AcrA [276]. This tripartite complex works synergistically with the outer membrane barrier because it allows the efflux of drugs into the external medium from which the drugs must pass through the outer membrane barrier to reenter the bacterial cells [277]. The intrinsic resistance of most gram-negative bacteria is due to the physical exclusion of the mostly lipophilic drugs like penicillin G, linezolid, oxacillin, nafcillin, macrolides, cloxacillin by the outer membrane. With the outer membrane disrupted $E$. coli are vulnerable to these agents [278]. Inactivating AcrB in the presence of an intact outer membrane also results in sensitivity of E. coli to these drugs [279].

AcrB of E. coli has extremely wide substrate specificity for common antibiotics and dyes, detergents and even solvents. AcrD, on the other hand, specifically pumps out aminoglycosides. There are several chromosomal genes coding for these pumps and their expressions are increased in response to regulators or due to mutations so that the bacteria can become more resistant to almost all antimicrobial agents in a single step.

Drugs can also be captured from the periplasmic space by at least some RND pumps. Consequently, these pumps not only prevent the entry of drugs but also extrude 
agents, such as $\beta$-lactams that target periplasmic substrates. Carbenicillin, a drug impermeable to the inner membrane due to having two carboxylate groups is another example of an RND substrate [277]. Large periplasmic extramembrane and mutantions in this domain show altered substrate specificities [280]. The RND tripartite pumps allow collaborative drug-export with simple pumps that can extrude their substrate drugs only into the periplasm [281].

The structure of the outer membrane channel TolC exists as a trimer with a single $\beta$ barrel and a remarkably long $(\sim 70-\AA)$ periplasmic extension in the form of long $\alpha$-helical bundles [282]. Similarly, AcrB exists as a trimer with a periplasmic portion as large as its transmembrane portion and the top of the periplasmic domain had a dimension similar to the a-helical bundle of TolC [283]. Co-crystallization of AcrB with various drugs suggested that drugs could diffuse through the enclosed area in the pump, the vestibule and be captured into the central cavity so they can leave the cell [284]. Also, crystallizing mutants of the AcrB altered at one of the putative residues involved in proton translocation showed only minimal conformational alterations in the periplasmic domain. This result suggested that the central cavity is the main site of substrate binding followed by its extrusion [285]. In addition, two substrates, minocycline, and doxorubicin, cocrystalized with AcrB were found in somewhat different parts of the cavity, which explains the range of AcrB specificity. For example, the pocket with hydrophobic surface in AcrB favors lipophilic substrates. In the bound state, an expanded binding pocket with several aromatic residues was found nearby the enclosed periplasmic domain that opens a pathway between the binding pocket and the vestibule. In the efflux mode, the binding pocket was shown much narrower and the pathway to the vestibule was closed as expected for peristaltic movement [286]. Therefore, the opening and closing of the large external cleft in the periplasmic domain is proposed to confer a cyclic motion. By homology modeling it was shown that the hypothetical binding 
pocket of AcrD was indeed the binding site of AcrB, pumping out basic and hydrophilic aminoglycosides, and it contains many electronegative atoms in the binding pocket [287]. Also, in the same study, AcrD was shown that it could capture aminoglycosides from both periplasm and cytoplasm and export out of the bacteria [287].

In clinic, RND pumps make major contributions to both intrinsic and elevated resistance to relevant pathogens [288-290]. For example, AcrA overproduction was confirmed responsible for high-level of fluoroquinolone resistance in clinical isolates of $E$. coli [291]. Fluoroquinolone resistance in $P$. aeruginosa is rapidly increasing and efflux pumps are major contributors in most cases [290]. Lately, adding disinfectants to household products like soaps has become increasingly popular. However, this may bring another huge problem because these compounds may place evolutionary selection pressure for pump overproducing microbial mutants that could become pathogenic [292]. Therefore, people need to use detergents with precaution as they do for antibiotics.

Fifth category: The MATE (multidrug and toxin extrusion) family of efflux transporters includes NorM of Vibrio parahaemolyticus, a protein with 12 transmembrane helices, pumps out mainly hydrophilic fluoroquinolones, cationic drugs and ethidium in

exchange for the influx of sodium ions [293]. Also, a similar MATE family pump was found in Bacteroides fragilis [294]. The E. coli homolog of NorM, YdhE was also shown to pump out aminoglycosides [295].

\section{Physiological Functions of Efflux Pumps}

Because the genes that encode most efflux pumps that contribute to drug resistance were already present in the chromosomal of bacterial strains isolated long before the antibiotic era, the RND-type multidrug pumps could not have primary functions against 
antibiotics. Therefore, multidrug efflux pumps must have had natural physiological functions other than the extrusion of exogenous chemicals.

The roles of some pumps are known for normal bacterial physiology and for others a normal physiological role is speculated based on the operon they are in or some other feature or combination of features of the gene or protein. For example, the MFS pump in $B$. subtilis was found in an operon that detoxifies spermidine, implying that MFS pump had role in that detoxification pathway [296]. Secondly, AcrB was shown to protect E. coli against bile salts and detergents, which are highly abundant where the bacteria live [297]. Similarly, Pseudomonas putida can tolerate high concentrations of solvents and was shown to achieve this via RND transporters optimized for the efflux of solvents [298]. Also, as their

name includes, RND transporters are involved in the secretion of nodulation factors or lipochitin oligosaccharides by Rhizobium [299]. Lastly, RND pumps play role in protecting the bacteria against plant secondary metabolites [263].

In M. tuberculosis there are many examples of RND transporters, two of which are exemplified here. Firstly, MmpL7 is involved in the export of complex, apolar lipid, phthiocerol dimycocerosate [300] and secondly, MmpL8 exports 2,3-diacyl-a, $\alpha^{\prime}$-trehalose-2'sulfate, a precursor of sulfatides [301].

\section{Multidrug Resistance in Cancer Therapy}

As in the case of bacteria, cancer cells can develop multidrug resistance (MDR) and it is still one of the major problems in clinical anticancer therapy despite the recent advances in cancer treatment. MDR is mainly facilitated by the members of the adenosine triphosphate (ATP)-binding cassette $(A B C)$ superfamily proteins including P-glycoprotein (Pgp or MDR1) [249], MRP family members [302] and MXR (ABCG2) [303]. 
$A B C$-transporters were the first transporters identified as critical in multidrug-resistant phenotype in an in vitro model. This membrane protein family includes the well-known drug efflux pump MDR1/P-glycoprotein (MDR1/P-gp or ABCB1). The MDR1/P-gp was isolated in 1979 [304] and later found to be encoded by the MDR1 gene, the ABCB1 gene [305]. A huge number of other multidrug-resistant human and mammalian cells were found to be over-expressing the MDR1 [306-308]. In these cells, the MDR1 functions as a drug efflux pump that causes a dramatic decrease in intracellular drug concentration; thus, the extruded antineoplastic agents can no longer act on their target molecules. The MDR phenotype is characterized by a typical cross resistance pattern against a variety of anticancer agents like vinca alkaloids, epipodophyllotoxins, anthracyclines, or taxanes, and the reversibility by verapamil and cyclosporin A derivatives [257, 309-311]. Beside the cytoplasmic membrane, these efflux pumps were found in both the nuclear membrane [312] and vesicular membranes [313]. So far, 49 different transporter proteins have been identified as $A B C$ transporters in humans [314].

In general, ABC-transporters function as ATP-dependent drug efflux pumps and have a highly conserved consensus sequence designated as the ATP-binding cassette $(A B C)$ or nucleotide binding domain (NBD). The ABC domain contains two short peptide motifs, both involved in ATP binding and common in all nucleotide-binding proteins [315]. Another consensus sequence is called the $A B C$ signature [316] and is unique in $A B C$ domains. The ABC-transporter is different from the other NBD containing proteins because it includes a hydrophobic transmembrane domain (TMD) that is usually composed of at least six transmembrane (TM) a-helices. These TMDs are the specificity determinants for the substrate to be transported. The human MDR1/P-gp consists of two TMD and two NBD within 1280 amino acids residues. It has been shown to be intrinsically over-expressed in tissues such as colon, kidney, breast and results in a primary multidrug-resistant phenotype 
$[309,317,318]$. These data indicate that MDR1/Pgp-mediated MDR is the primary cause of poor treatment of MDR1/P-gp-positive tumors

In 1992, ABC-transporters of the MRP (ABCC) subfamily were identified as the mediators of a multidrug-resistance [319]. In addition to the two TMD/two NBD core of MDR1/P-gp, MRP1, 2, 3, 6, and 7 have an additional $\mathrm{NH}_{2}$-proximal TMD0 domain consisting of 5 TM a-helices. MRP4, 5, 8, and 9 lack the TMD0 domain. MRP1- 8, but not MRP9, have been determined to export any type of anticancer compound. MRPs can pump out organic anions and several Phase II metabolic products including anthracyclines, Vinca alkaloids, Epipodophyllotoxins, and methotrexate [320]. Although MRP1 expression was analyzed in breast cancer [321] and ovarian cancer [322], the role of MRP1 in clinical MDR remains to be elucidated due to inconsistent data between MRP1 expression and its clinical outcome.

MRP2 or ABCC2 was originally identified as a platinum drug transporter [323], but it also has the ability to extrude MRP1 substrates [320]. During normal physiology, it transports bilirubin glucuronide into the bile canaliculi of the liver from the apical membranes of hepatocytes and disorders of MRP2 activity results in accumulation of bilirubin glucuronide and unconjugated bilirubin in the liver known as Dubin-Johnson syndrome (DJS). MRP2 expression was detected in breast cancer [324] and ovarian carcinoma [325]. MRP3 (ABCC3) has less affinity for anticancer agents than MRP1 and 2 and it only confers low levels of resistance to Epipodophyllotoxins and Vinca alkaloids [326]. In contrast to MRP1 and 2, MRP3 does not require glutathione for drug transport. MRP3 is usually expressed at the basolateral surface of hepatocytes [327], where it may pump organic anions into the sinusoidal blood. Although MRP3 was detected in breast cancer [328], primary ovarian cancer [329], pancreatic carcinoma [330], and glioma [331], so far no link between MRP3 and clinical parameters has been demonstrated. 
MRP4, 5, and 8, (ABCC4, $A B C C 5, A B C C 11)$ mainly transport purine and pyrimidine nucleosides as well as their cyclic derivatives [326, 332, 333]. The MRP4 transcripts predominantly localized in prostate and kidney whereas MRP5 is generally seen in urogenital tissue [334][51] although both can be detected in various tissues [302]. Also, MRP8 is assumed to be expressed widely in human cancer tissues [332].

MRP6 (ABCC6) can act as a drug efflux pump and mediates low levels of resistance to some epipodophyllotoxins, anthracyclines and cisplatin, in vitro [335]. Its physiological substrates are hydrophobic organic anions including cyclic peptides and glutathione conjugates but not glucuronate conjugates. In contrast to the early studies indicating that MRP6 expression was limited primarily to the liver and, to a lesser extent, to the kidney, more recent data showed a widespread distribution pattern of MRP6 expression [336]. So far, no clear MRP6 levels could be detected in frozen sections of human tumor samples from different tissues, indicating that the contribution of MRP6 to drug-resistance may be rather limited [337]. However, MRP7 can be found in cancer cell lines to mediate resistance against taxanes and Vinca alkaloids [332, 338].

$\mathrm{BCRP}$ (breast cancer resistance protein) or ABCG2 is the most important drug efflux pump in the context of anticancer drug resistance. It was identified by three independent studies [339-341]. The pump protein of $72 \mathrm{kDa}$ has a NBD and a TMD probably to form homodimers in an active transport complex [342]. BCRP expression was found in various normal tissues including placenta, colon, small and large intestine, sebaceous glands, islet and acinar cells of the pancreas, hepatocytes and biliary canaliculi, alveolar pneumocytes, breast tissue, venous endothelium, and in capillaries, zona reticularis of the adrenal gland, cortical tubules of the kidney, and prostate epithelium, suggesting a protective role for ABCG2 [343, 344]. It was shown that BCRP secreted xenotoxins, including carcinogens 
and anticancer drugs, into milk [345]. BCRP was detected in AML and acute lymphoblastic leukemia (ALL) [346, 347].

Other human $\mathrm{ABC}$-transporters were found to transport antineoplastic agents in vitro. Over-expression of $A B C 2(A B C A 2)$ contributes to estramustine resistance [348], $A B C 3$ (ABCA3) was associated with resistance to doxorubicin [349]. Over-expression of both TAP1 (transporter associated with antigen presentation) and TAP2 results in increased resistance to mitoxantrone or etoposide [350, 351]. Similarly, in vitro studies indicated that MDR3/P-gp (ABCB4) contribute to resistance to taxanes and Vinca alkaloids [352] in coordination with BSEP (ABCB11) that is also involved in resistance to taxanes [353, 354]. Overexpression of MTABC3 (ABCB6) was determined in cell lines resistant to cisplatin [355] or camptothecin [356].

Doxorubicin transport and chemo-resistance in human melanoma cells is mediated by $\mathrm{ABCB} 5$ [357]. A subpopulation of tumor-initiating cells having cancer stem cell (CSC)like characteristics in a human malignant melanoma was shown to express ABCB5 [358]. ABCB5-positive tumor cells were correlated with clinical melanoma progression having greater tumorigenic capacity than cells negative for $A B C B 5$ in melanoma patients. $A$ monoclonal antibody against ABCB5 induced antibody-dependent cell mediated cytotoxicity

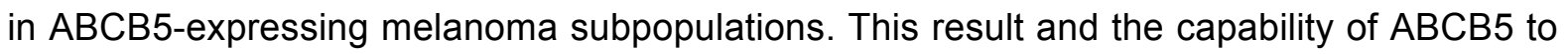
mediate drug resistance suggest that this transporter may have important implications for clinical management of melanoma patients.

Unfortunately, the exact role of many members of the ABC-transporter family in drug resistance is still unknown or not fully understood. It is not even known if the same ABCtransporter is active from the beginning of drug treatment to the end. Therefore, the possibility that the high expression of all three pumps, MDR1/P-gp, MRP1 and BCRP, that 
export mitoxantrone are taken into consideration during the mithridatism period in response to mitoxantrone [359].

In cancer cell lines, increased expression of other drug resistance associated transporters were detected in addition to the expected well characterized ABC-transporters. These results suggest that drug resistance in cancer cells can be mediated through several ABC-transporters of unknown function [360, 361]. In acute lymphoblastic leukemia (ALL) and breast cancer patients, the expression of most ABC-transporters was detected and comparison of their expression levels in drug treated and untreated tumors showed an unexpected similarity [362]. Overall, the results suggest that drug-resistant phenotypes have shown high expression level of ABC-transporters.

A non-ABC-transporter, RALBP1 (RLIP76), which is a $76 \mathrm{kDa}$ Ral-binding Rho/RacGAP and Ral effector protein, can transport structurally unrelated amphiphilic chemotherapeutic drugs such as doxorubicin and glutathione-electrophile conjugates [363, 364]. RALBP1 transports xenobiotics in an ATP-dependent manner as in the case of the ABC-transporters, but it lacks sequence homology with the ABC-transporter family members. This transporter has multiple motifs including tyrosine kinase phosphorylation sites, two distinct ATP-binding domains, a H(+)-ATPase domain, a Rho/Rac GAP domain and a Ral effector domain binding motif. As for many of the other efflux pumps, so far no data are available as to whether this non-ABC-transporter has an impact on clinical drug resistance, despite a current study demonstrating RALBP1 expression in human tumors such as ovarian cancer [365].

\section{Inhibitors of Efflux Pumps}

In cancer treatment: MDR pump antagonists have been developed and used in cancer treatment in order to reverse the effects of efflux pumps, [366]. Cyclosporine A, 
verapamil, biricodar citrate, valspodar, tariqudar and quercetin are some of these drugs that were successfully used as MDR antagonists to increase the efficacy of the antineoplastic agents,

The development of Pgp inhibitors has a history of about 30 years. In the 1980s, cyclosporine A, verapamil and tamoxifen were identified as Pgp substrates and developed to inhibit Pgp. However, their mechanism of action was to compete with the cytotoxic compounds. Thus, a high concentration was needed to observe their effect, which in turn caused serious toxicity in vivo [367]. In addition, these compounds were later identified as substrates of other transporters and inhibitors of enzymes that could have been the reason for unexpected high toxicity in vivo [368].

Second-generation Pgp modulators have been developed to decrease the toxicity of the first Pgp antagonists. Biricodar citrate, a pipecolinate derivative, binds Pgp with a very high affinity and reverses its activity [369]. Also, valspodar (PSC 883), a derivative of cyclosporin $D$ is 20 -fold more effective than cyclosporin $A$ [370]. These second-generation drugs had much better inhibitory activity than the early-generation Pgp inhibitors. However, these second-generation drugs unfortunately still had serious limitations. For example, valspodar was identified as the inhibitor of cytochrome P450 3A4 (CYP3A4), which also metabolizes paclitaxel and vinblastine [371]. This created a serious problem for the patients who used paclitaxel and vinblastine. The biochemical analysis showed that these patients had higher serum concentrations of these cytotoxic agents because valspodar was acting as an inhibitor of CYP3A4 [372]. As a result, there was a need for the development of new drugs.

Third-generation Pgp modulators, tariqudar [373], the cyclopropyldibenzosuberane zosuquidar [374], laniquidar [375], have been developed that do not have the side effects of the second and early-generation MDR pump antagonists. They were required to have 
better selectivity and specificity for the pump and lower potential to interact with CYP3A4. Tariqudar (XR9576) was one these third generation drugs and it specifically and noncompetitively bound to the pump with better affinity than the intrinsic substrates of the pump $[373,375]$. It also exhibited an enhanced clearance time compared to cyclosporine A [376], and it did not affect the activity of CYP3A4. The other two compounds share similar properties of better selectivity, specificity and also less chance to affect off-target activities. Therefore, third-generation drugs offer better advantages over the drugs developed earlier. However, there are still more MDR antagonists under development.

More recently, quercetin, a flavonoid derivative, was used as a chemosensitizer to modulate cancer multidrug resistance (MDR) [314\{Chen, \#3295]. It is ubiquitously found in onion, apples, tea and berries and shown to have antioxidative, anti-inflammatory and vasodilating effects [377]. Biochemical and biophysical studies indicated that it had also the ability to sensitize multiple MDR pumps including Pgp [378, 379], BCRP [380] and MRP [381] to several chemotherapeutic anticancer drugs. Also, in vivo studies indicated that it could increase the bioavailability of tamoxifen, paclitaxel, etoposide, diltiazem and fexofenadine [314]. The mechanism of its action was shown to competitively inhibit the MDR pumps by binding to the ATP binding site or substrate binding site [382, 383]. Ongoing clinical trials with third-generation inhibitors, quercetin and some unmentioned compounds are hoped to result in better survival rates in patients with cancer.

To treat Bacterial Infections: The clinical significance of antibiotic resistance has prompted the search for efflux pumps inhibitors (EPIs), which can be used against multidrug resistant bacteria. These EPIs include both natural and synthetic molecules. Reserpine and berberine are isolated from plants and used as natural products to inhibit MDR pumps. Synthetic compounds include the hybrid compounds of berberine, phenylalanyl arginyl $\beta$ naphthylamide (PAßN), arylpiperazine derivatives and derivatives of the expelled antibiotics. 
Plants have been shown to produce several of these natural inhibitors. Reserpine is one of the first EPIs, which was isolated from dried root of Rauwolfia serpentina. It has demonstrated an ability to potentiate antibiotics in Gram-positive bacteria by inhibiting the MDR pumps [384]. Also, another plant species called Berberis fremontii was found to simultaneously produce an antibiotic berberine and an MDR inhibitor 5'methoxyhydnocarpin (5'-MHC). 5'-MHC blocks the NorA pump to overcome the problem of antibacterial resistance but 5'-MHC alone had no antimicrobial activity. Later 5'-MHC was found to potentiate the antibiotic action of berberine and other NorA substrates against $S$. aureus [385]. The latter has aided the development of synthetic EPIs.

One of the synthetic compounds was obtained by conjugating berberine with an inhibitor of major facilitator MDRs, INF 55. Then, it was used as a successful hybrid compound with activity against resistant bacteria [386]. Recently, the 13-substituted berberine-NorA inhibitor hybrid was synthesized via chemically linking berberine with the NorA inhibitor, 5-nitro-2-phenyl-1H-indole. This new compound had 382-fold more activity than the parent antibacterial berberine against $S$. aureus [387].

Another synthetic EPI is called phenylalanyl arginyl $\beta$-naphthylamide (PA $\beta N)$. It is a substrate for the MexAB-OprM pumps and was shown to increase the activity of levofloxacin in resistant $P$. aeruginosa strains [388]. In addition, high concentration of PAßN was able to disrupt the membrane integrity that directly influences the drug penetration [388]. Later, a better compound called MC-04, 124 was identified with better stability and lower toxicity than PAßN against $P$. aeruginosa. Finally, to inhibit MexAB-OprM efflux pump, the pyridopyrimidine derivatives have extensively been assayed to improve their inhibitory activities against the $P$. aeruginosa [389]. Nevertheless, these compounds had intrinsic problems like low solubility, low stability and high affinity towards the serum proteins. New derivatives of these compounds are under development with 
There have been EPIs developed against E. coli with high expression of efflux pumps. For example, 3-arylpiperidine, an arylpiperazine derivative, helped to reverse the effect of MDRs and potentiate novobiocin in E. coli [390, 391]. Also, bacteria exposed to 1(1-naphthyl)piperazine and 1-(1-naphthylmethyl)-piperazine have got more susceptible to levofloxacin, clarithromycin, linezolid, oxacillin, rifampin, chloramphenicol and tetracycline [391]. Finally, scientists successfully developed efflux pump inhibitors by using derivatives of the expelled-antibiotics. For example, tetracycline-analogues were used to restore the activity of tetracycline activity against resistant bacteria [392]. Based on the developed antagonists, the main mechanism of inhibition is so much dependent on molecular volume, charge and the position of substituted groups that these new compounds can induce steric hindrances that collapse the drug transport. Therefore, inhibition of efflux pumps can restore the drug susceptibility of resistant strains and thus some of the old antibiotics can again be effective. In spite of these developments in EPIs, none of the above-mentioned inhibitors are yet in clinical use. 


\section{MECHANISMS OF RESISTANCE TO AMINOGLYCOSIDES}

Aminoglycosides are compounds characterized by the presence of an aminocyclitol ring bound to aminosugars. Their bactericidal activity is attributed to the irreversible binding to the ribosomes so they exhibit a broad antimicrobial spectrum. Aminoglycosides have been extensively used against aerobic and facultative aerobic Gram-negative bacilli and some Gram-positive staphylococci but they are not active against anaerobes and rikettsia.

The misuse of aminoglycoside antibiotics has resulted in bacteria that have evolved mechanisms for eliminating the effects of aminoglycosides. Three major mechanisms of resistance to aminoglycosides have been recognized; ribosome alteration, decreased permeability and inactivation of the drugs by aminoglycoside modifying enzymes. Ribosome

alteration is not so common due to the fact that the function of the rRNA and ribosomes is central in the protein synthesis process which is so well-conserved across many generations that it cannot be impaired by the possibility of such structural alteration/s. However, aminoglycoside inactivation has the most clinical importance since the genes encoding aminoglycoside modifying enzymes can easily be transferred by plasmids and/or transposons. Each of these mechanisms is discussed below with common examples given.

\section{Ribosome Alteration}

The first mechanism to get aminoglycoside resistance is through modifying the ribosomes. Once there occur modifications or mutations either in rRNA or in the ribosomal proteins, where aminoglycosides bind, they lower binding affinity of the aminoglycoside antibiotics. For example, alterations in ribosomes resulted in problems in treating Mycobacterium tuberculosis with streptomycin, which was primarily used for tuberculosis treatment. Streptomycin functions by binding to $16 \mathrm{~S}$ rRNA in ribosomes and all streptomycin-resistant $M$. tuberculosis strains were found to have point mutations leading to 
alterations in the streptomycin binding site. These changes resulting in streptomycin resistance were identified in both $16 \mathrm{~S}$ rRNA and ribosomal proteins, S4, S12 and S5 of the 30 S ribosomal subunit [393, 394].

In addition to mutations in the ribosomes of $M$. tuberculosis, mutations in the ribosomal protein L22 confer resistance to erythromycin in Thermus thermophilus. The Xray crystal structure of this protein indicated that the mutations causing resistance to erythromycin were found on a beta-hairpin loop of L22 [395]. Also, it was postulated that this region was in close proximity to the erythromycin-binding site near the peptidyl transfer center [395].

Methylation of $16 \mathrm{~S}$ rRNA at N7 of guanine residues can result in aminoglycoside resistant strains, as well. However, this is mainly confined to aminoglycoside-producing organisms and it gives high-level of resistance to the actions of these antibiotics [396-398], Therefore, these organisms can still survive in the presence of the aminoglycosides via modifications as in the case of mutations in the ribosomes other aminoglycoside resistant organisms.

\section{Decreased Permeability and Active Extrusion}

The second way to obtain aminoglycoside resistance is via decreasing permeability of the aminoglycosides $[288,289]$. Absence of or alteration in the aminoglycoside transport system, inadequate membrane potential, modification in the lipopolysacchaccarides (LPS) phenotype can be given as mechanisms for decreasing the aminoglycoside permeability [399]. It was also shown that these mechanisms were chromosomally mediated thus result in cross-reactivity to all aminoglycosides and that why it was highly significant in the clinic [400]. 
Some strains of Pseudomonas aeruginosa and other Gram-negative bacilli can be given as examples which obtained aminoglycoside resistance due to a transport defect, inadequate membrane potential or membrane impermeabilization [401]. Active efflux of the aminoglycosides is the other mechanism how some organisms can get resistance as it was explained in detail in the previous section. In sum, bacteria can get resistant to the aminoglycosides with decreasing the permeability of the aminoglycosides which in turn lowers the amount of available aminoglycoside in bacteria.

\section{Inactivation of aminoglycosides}

Inactivation of aminoglycosides is achieved by the expression and action of three major classes of aminoglycoside-modifying enzymes based on their modification type: acetyltransferases (AAC), nucleotidyltransferases or adenyltransferases (ANT), phosphotransferases (APH). These modifications occur through $N$-acetylation, $\mathrm{O}-$ nucleotidylation and O-phosphorylation as shown in Figure 2. In each class, there are enzymes with different side chain specificities for aminoglycoside modifications. For example, there are four nucleotidyltransferases [ANT(6), ANT(4'), ANT(3") and ANT(2")], seven phosphotransferases $\left[\mathrm{APH}\left(3^{\prime}\right), \mathrm{APH}\left(2^{\prime \prime}\right), \mathrm{APH}\left(3^{\prime \prime}\right), \mathrm{APH}(6), \mathrm{APH}(9), \mathrm{APH}(4)\right.$ and $\left.\mathrm{APH}\left(7^{\prime \prime}\right)\right]$ and four acetyltransferases [AAC(2'), $\mathrm{AAC}\left(6^{\prime}\right), \mathrm{AAC}(1)$, and $\left.\mathrm{AAC}(3)\right]$. Also, there are bifunctional enzymes like $\mathrm{AAC}\left(6^{\prime}\right)-\mathrm{APH}\left(2^{\prime \prime}\right)$, which can sequentially acetylate and phosphorylate its substrates $[402,403]$ and ANT(3")-AAC(6'), which can acetylate the 6'amine of kanamycin-A and adenylate the 3"- and 9-hydroxyl groups of streptomycin and spectinomycin [404].

Structural studies indicated that mainly the same electrostatic interactions that drove the evolution of aminoglycosides for binding to the ribosomal sites also appeared to be similarly involved in evolution of the resistance enzymes [405-407]. The presence of acidic 
groups in the aminoglycoside binding sites appears to be a recurring theme in aminoglycoside-modifying enzymes. For example, the active site of the enzyme APH(3')IIla has excessive negative charge distribution that provides favorable interactions with positively charged aminoglycosides [406, 408]. Also, the amines in aminoglycosides, important for binding to $16 \mathrm{~S}$ rRNA A-site, appeared to be significant for recognition of the drugs by the resistance enzymes [409].

ANT(2")-la is frequently associated with clinical resistance in Gram-negative organisms to gentamicin and tobramycin [410]. This enzyme transfers mononucleotide to the aminoglycosides as shown in Figure 2 and it shows a broad substrate specificity [411]. Mechanistically, its rate limiting step was shown as the release of adenylated aminoglycoside [412]. The structure of ANT(2") has not been known, yet.

However, the structure of ANT(4')-la in complex with kanamycin and the nonhydrolyzable ATP analogue (AMPCPP) has shed light on the details of the mechanism of action for the aminoglycoside nucleotidyltransferases (ANTs) [407]. For example, the active enzyme was found as a dimer with two heterodimeric catalytic sites. These catalytic sites contain residues from one monomer predominantly contributing to the magnesium and ATP binding as well as with residues from the other monomer for aminoglycoside binding [407, 413]. Acidic residues generate a negatively charged surface patch to guide the positively charged aminoglycosides to the active site to facilitate their binding. For example, a glutamic acid (Glu145) residue functioned as the general base to deprotonate the incoming aminoglycoside thereby increasing the nucleophilicity of the attacking 4' hydroxyl group. The substrate promiscuity of this class of enzymes can also be attributed to the abundance of negatively charged hydrogen acceptor groups which can therefore accommodate a broad spectrum of positively charged aminoglycoside substrates. Also, a positively charged residue, Lys149, was in close proximity with the a-phosphate group of ATP and it possibly 
increased electrophilicity of this phosphate, making it more susceptible to nucleophilic attacks. Such a direct attack mechanism was shown to be consistent with the results of stereochemical analysis of the ANT(2")-la reaction with tobramycin, as well [414].

Bioinformatics studies revealed that part of the ANT(4') fold was also present in the crystal structure of eukaryotic DNA polymerase $\beta$ (Pol $\beta)$, an enzyme catalyzing Onucleotidyl transfer, despite their minimal sequence similarity with ANTs [415]. This structural similarity implied that these two nucleotide monophosphate transferases are evolutionary related, suggesting the existence of a Polß-like nucleotidyltransferase superfamily. Later studies indicated that this superfamily involved enzymes, which took role in antibiotic resistance (ANT(4')-la), DNA replication and repair (DNA Polß), chromatin remodeling (yeast Trf4p) and signal transduction (GInD) [416]. This superfamily is wellconserved through all three kingdoms of life and has members in bacteria, archaea and eukaryotes.

Aminoglycoside acetyltransferases (AACs) are the second type of enzymes which catalyzes the acetyl-CoA dependent $N$-acetylation of aminoglycosides as shown in Figure 2 and Figure 3. They can modify amino groups on both the aminoglucose ring [AAC(6'), $\left.A A C\left(2^{\prime}\right)\right]$ and the 2-deoxystreptamine ring $[A A C(1), A A C(3)]$. Both $A A C\left(6^{\prime}\right)$ and $A A C(3)$ were found as common resistance elements in Gram-negative bacteria [410]. Also, in Gram-positive bacteria, $\mathrm{AAC}\left(6^{\prime}\right)$-le was found as the major acetyltransferase associated with clinically important gentamicin resistance [410]. Acetylation of the amine groups directly results in unfavorable steric clashes and electrostatic attraction in the A-site RNA [417]. As mentioned earlier, there are also bifunctional enzymes one of which is $A A C\left(6^{\prime}\right)-A P H\left(2^{\prime \prime}\right), N-$ terminal portion of which was formed by $A A C\left(6^{\prime}\right)-l e$ [418]. It was later found in Gram-positive Staphylococci, Enterococci, and Streptococci [419]. 
Structural studies indicated the positioning of the aminoglycosides butirosin-A and isepamicin when bound to AAC(6')-li in NMR experiments [420]. These studies suggest that the 4,5- and 4,6-disubstituted aminoglycosides bind differently to AAC(6')-li. For example, 4,5 disubstituted aminoglycoside butirosin-A has only one binding mode whereas 4,6disubstituted aminoglycoside isepamicin has two alternative conformations, one of which may be a non-productive complex.

Also, two available crystal structures resolved the position and orientation of the CoA and $\mathrm{AcC}$ A groups for $\mathrm{AAC}(3)-\mathrm{la}$ and $\mathrm{AAC}\left(6^{\prime}\right)$-li, respectively. A negatively charged surface patch, analogous to the aminoglycoside binding region of ANT(4')-la, was found next to the CoA sulfur atom in both $\mathrm{AAC}(3)$-la and $\mathrm{AAC}\left(6^{\prime}\right)$-li. Therefore, it is probable that the two AAC enzymes share similar strategy to interact with aminoglycoside substrates with the ANT enzymes. The common structural motif found in AAC(3)-la and AAC(6')-li was also present in other enzymes like yeast histone acetyltransferase (yHat1) and the fungal $\mathrm{N}$ myristoyltransferase (Nmt) [421, 422]. These observations confirmed that both AAC(3)-la and $A A C\left(6^{\prime}\right)$-li belong to the GCN5-related N-acetyltransferase (GNAT) superfamily [423].

Aminoglycoside phosphotransferases (APHs) catalyze the ATP-dependent Ophosphorylation of aminoglycoside hydroxyl groups as shown in Figure 2 and Figure 3. They are found in both Gram-negative and Gram-positive bacteria. In the Gram-positive cocci, the bifunctional enzyme $\mathrm{AAC}\left(6^{\prime}\right)-\mathrm{APH}\left(2^{\prime \prime}\right)$ has the main mechanism for gentamicin resistance [419]. Additionally, the C-terminal $\mathrm{APH}(2$ ") activity of this enzyme has a broad substrate specificity and also has the capacity to phosphorylate aminoglycosides on the 6aminohexose and 3-aminohexose rings.

The $\mathrm{APH}\left(3^{\prime}\right) \mathrm{s}$ are the most abundant class of APH enzymes that modify both the neomycin and kanamycin-class aminoglycosides. APH(3')-IIla was shown to have a broad substrate specificity with the capacity to modify many aminoglycosides with an exclusion of 
the clinically important gentamicin-C. Kanamycin-class aminoglycosides are phosphorylated on the 3'-hydroxyl, whereas neomycin-class antibiotics on both 3'- and 5"positions. Even, some of these groups can be readily di-phosphorylated. The crystal structure of $\mathrm{APH}(3)-$ IIla in complex with ADP indicated the nucleotide binding site was deep in the active site with only the terminal phosphate group being solvent accessible. This indicated that the ADP-release was the rate limiting step of the reaction [406].

Very similar to the ANTs and AACs, aminoglycoside binding pocket of APHs contains acidic residues, which supplies electrostatic potential for guiding and binding a broad spectrum of aminoglycosides. The crystal structure also revealed the order of substrate binding and product release. ATP binds prior to the aminoglycoside, and release of the phosphorylated aminoglycoside precedes that of ADP, because binding of an aminoglycoside obstructs the entrance to the nucleotide binding pocket [406].

Nearly $50 \%$ of these enzymes exhibited a fold identical to that of protein kinases despite the lack of amino acid sequence similarity (<3\%) [424]. Even, APH(3')-IIla was found more closely related to the eukaryotic protein kinases than type II phosphatidylinositol phosphate kinase (PIPKII) [425]. This similarity between aminoglycoside phosphotransferases and protein kinases suggests an evolutionary link between these two classes of proteins. Therefore, it was not surprising that they showed similar sensitivity to the inhibitors. For example, the flavanoids quercein, genestein and the isoquinoline sulfonamides, which inhibit protein kinases as competitive inhibitors of ATP binding, exhibited similar effects on the APHs [426].

Modified aminoglycosides lose their ability to bind to the target A-site due to unfavorable steric and/or electrostatic interactions as a result of the action of AACs, ANTs and APHs. For example, acetylation of the amine group at position 3 on paromomycin results in unfavorable steric clashes and electrostatic attraction in the A-site RNA [417]. 
Another example is the phosphorylation at the 3' position by the action of APH (3') in which the phosphate group at the 3' position would interfere with the binding due to the repulsive electrostatic and steric interactions between the phosphate backbone of the rRNA, resulting in very poor binding [417]. Tobramycin and gentamicin-C are susceptible to five or six of these aminoglycoside modifying enzymes as summarized in Figure 3 . However, a kanamycin-A derivative with acylated amino group at position 1 by 4-amino-2hydroxybutyrate, amikacin was saved from the enzymatic modification at multiple positions. Also, the aminohydroxybutyryl modification at position 1 of amikacin appears to fit well into the extended cleft and the amino group in this moiety may also enhance binding of amikacin to rRNA through electrostatic interactions [417]. Consequently, greater than $80 \%$ of the gentamicin-resistant members of Enterobacteriaceae and 25 to $85 \%$ of Pseudomonas aeruginosa-resistant strains were found sensitive to amikacin [427, 428].

In conclusion, the structural information of all three classes of aminoglycoside modifying enzymes has detailed the mechanism of resistance to this important class of antibiotics. Therefore, the combination of both structural and functional analysis should enhance the rational design of novel inhibitors of these enzymes, which can be identified from other protein kinase inhibitors. Potent and selective inhibitors of these enzymes have the potential to block aminoglycoside resistance in vivo and therefore could extend the clinical use of aminoglycosides. Finally, rational design of new aminoglycoside derivatives that are not susceptible to the enzymes can be synthesized and tested. 


\section{APPROACHES TO PREVENT DRUG RESISTANCE}

Antibiotic resistance is a huge concern for treating disease. The increasing number of antimicrobial resistant strains in the hospitals makes it even harder to fight bacterial infections. These resistant bacteria easily multiply and spread their antibacterial immunity to others through lateral gene transfer. We can prevent the development of antibiotic resistance by three main ways; 1) simultaneous application of multiple drugs, 2) minimizing our use of antibiotics or 3) by developing alternative ways to treat bacterial infections.

\section{Simultaneous Use of Multiple Drugs}

The chance of drug resistance can sometimes be minimized by simultaneous use of multiple antibiotics. This works because individual mutations can be independent and may only create resistance to one group of drug. Hence, if the individual mutants are killed by another in the mix of drugs, then mutations cannot accumulate to produce a resistant progeny. This strategy was used successfully against tuberculosis [429, 430]. The combination of isoniazid and ethambutol, which have two independent targets and modes of action, has been used to treat tuberculosis in many countries [430]. However, cross resistance where mutations confer resistance to two or more treatments can be problematic. Interventions in improving antimicrobial use and blocking transmission of resistant organisms are required to combat antimicrobial resistance.

\section{Reducing Antibiotic Use}

The main cause of antibiotic resistance is the overuse of antibiotics; thus, it is highly recommended to use antibiotics when only necessary. They should not be taken for a viral infection like a cold, a cough or the flu. This is because every time antibiotics are used, they create a selective pressure on bacteria that promotes the expansion of resistant strains [431]. However, one can minimize this selection with the proper use of antibiotics. 


\section{Alternative Ways to Combat Bacterial Infections}

The emergence of bacterial strains resistant to many available drugs pointed to the need for new therapeutic methods. These new therapeutic methods have included bacterial interference, phage therapy, bacterial vaccines, cationic peptides and cyclic D,L-a-peptides.

\section{Bacterial interference}

Bacterial interference can be achieved by inoculating hosts with nonpathogenic bacteria, which can prevent infection by pathogenic strains. The mechanism of bacterial interference is the competition of nonpathogenic bacteria with pathogenic bacteria for nutrients and adhesion receptors, necessary to establish infections and propagate diseases. Nonpathogenic bacteria may also attack the pathogenic strains through secretion of antimicrobial compounds. This treatment has had promising results in infections of the gut, urogenital tract, and wound sites. A major advantage is that these nonpathogenic species do not stimulate the host's immune system and do not promote selection for antibiotic resistance [432].

\section{Bacteriophage Therapy}

Bacteriophages are viruses known as natural killers of bacteria by directing the host to make their own viral proteins. Their first use as therapeutics was in prophylaxis against cholera, typhoid fever and dysentery [433]. Now phage therapy once again has come to the forefront due to emergence of multi-drug-resistant bacteria [434]. In this way, specific strains of pathogens can be targeted through manipulation of phage DNA. Exponential growth and natural mutational ability make bacteriophages great candidates for counter attacking bacterial resistance; however, the technology is still developing and may be a way off before seeing daily use. 


\section{Bacterial vaccines}

Developing bacterial vaccines has become a popular idea with the advent of complete microbial genomic sequencing and with the understanding of virulence regulatory mechanisms [435]. By scanning the bacterial genome, drugs can be developed through target-based approaches. Also, protein sequences can be identified to stimulate a protective immune response against specific bacterial strains. Regulatory genes that control virulence protein production are excellent vaccine candidates for priming the human immune system or inhibiting virulence production [435]. Bacterial genomics can also be used to detect conserved sequences from bacterial species and strains worldwide. Therefore, this technology can yield clinical vaccine candidates.

\section{Cationic peptides}

Cationic peptides are either found throughout nature in the immune systems of bacteria, plants, invertebrates and vertebrates and have been used to kill bacterial cells. They have both hydrophobic and hydrophilic characteristics Cationic peptides have several mechanisms of action involving interaction with the bacterial cell membrane leading to cell death [436]. From a therapeutic standpoint, these proteins have a great promise, as they have co-evolved with bacteria yet have maintained the ability to target pathogenic bacteria.

\section{Cyclic D,L-a-peptides}

Cyclic D,L-a-peptides are synthetic and amphipathic molecules with cell membrane disruption capabilities. As their name implies these natural peptides are composed of alternating $D$ and $L$ amino acids with cyclic structures [437]. Some have been specifically engineered to target Gram-positive and Gram-negative bacterial membranes but not mammalian cell membranes. In contrast to any other known class of peptides, these peptides can self-assemble into flat ring shaped nanotubes, which specifically target and 
puncture bacterial cell membranes. Therefore, they increase membrane permeabilityand collapse transmembrane ion potentials causing rapid cell death [438]. The effectiveness of this class of materials was shown against lethal methicillin-resistant Staphylococcus aureus (MRSA) infections in mice [438]. These molecules can be used as complement to the current naturally derived antibiotics and help to combat a variety of existing and emerging antibacterial drug resistant infectious diseases. 


\section{AMINOGLYCOSIDES AS INTRACELLULAR RNA APTAMER TARGETS}

Aminoglycosides are one of the most important families of antibiotics and are mostly derived from Streptomyces spp. They are water soluble and their polycationic nature results in a very broad-spectrum of anti-bacterial activity against both Gram-positive and Gramnegative bacteria, mycobacteria and protozoa. Aminoglycosides contain 4 to 6 amino groups that are protonated at biological $\mathrm{pH}$ [417], which makes them very effective RNA targeting reagents. The neomycin-class of aminoglycosides, considered the most effective bactericidal agents, has been used clinically for many years.

Aminoglycoside antibiotics are composed of amino sugars linked to a deoxystreptamine ring (ring II) (Figure 4). The structurally conserved elements, among the neomycin-class aminoglycosides, rings I and II and the amino groups at positions 1 and 3 on the ring II (Figure 4) are essential for their RNA interactions [439]. Several analogues based on the neamine core have comparable or better binding affinities and antimicrobial activities than the parent aminoglycosides $[440,441]$. With these conserved chemical units, they exhibit high affinities for certain parts of the prokaryotic ribosomal RNA (rRNA) based on electrostatic and hydrogen-bonding interactions [186]. Biochemical and biophysical techniques have located the site of aminoglycoside binding to an adenine-rich internal loop at the 3'end of helix 44 in 16S rRNA in the 30S ribosomal subunit that consists of 16S rRNA and 21 proteins [132, 133]. Helix 44 binds aminoglycosides via its major groove and contacts the codon-anticodon complex with its minor groove and is therefore part of the aminoacyl-transfer RNA site (A-site) [186]. The consequence of aminoglycoside binding near the A-site, which is in the decoding region, is a conformational change in the 16S RNA, displacing two well-conserved adenine residues (A1492 and A1493) towards the minor groove [186, 439, 442-444]. This conformational change converts the A-site into a higher

affinity environment for tRNA-mRNA interaction, thereby lowering the rejection rate of near- 
cognate tRNAs [445]. Therefore, aminoglycosides inhibit translocation of the tRNA-mRNA from A-site to peptidyl-tRNA site (P-site) [446] and this causes a second consequence of misreading of the genetic code $[447,448]$. Besides causing misreading, paromomycin and neomycin can arrest assembly of the 30 S subunit formation resulting in the accumulation of a $21 \mathrm{~S}$ subunit, which is a possible precursor of the $30 \mathrm{~S}$ subunit [449]. In addition, they preferentially interact with prokaryotic ribosomes over the eukaryotic ones [450]. As a result, bacteria cannot synthesize vital proteins for their survival and they eventually die. Therefore, aminoglycosides have been successfully used against bacterial infections for over 50 years [408].

The continuing emergence of new strains of pathogens that are resistant to existing antibiotics drives the discovery of new antibiotics that overcome the resistance. One approach, which has seen some success, is to modify the existing antibiotics for more effective target binding. Aminoglycosides have been the subject of many modifications to enhance their interactions with the variety of RNA targets [451-456]. Some of these RNAaminoglycoside interactions have been structurally characterized. All of these NMR solution structures share general features with the aminoglycosides located in the major groove. This specific feature of helix 44 is not shared by the standard A-type RNA helix, which has too narrow and deep a major groove to bind aminoglycosides [457].

Nucleotides at the cores of aptamers play multiple structural roles by directly contacting the ligands and by participating in various mismatch and triplex Watson-Crick base pairing as well as other interactions with neighboring nucleotides in the aptamer. In this way, they determine the overall structure of the binding site, which, for aminoglycosides, is mainly a widened major groove with irregular helical domains. Such overlapping features of aminoglycoside binding sites resulting in similar binding site characteristics that may 
make it difficult to generate altered specificities for aminoglycosides within the same structural context [458].

Small RNA aminoglycoside interactions have been widely recognized over the past few decades $[450,459,460]$. In addition to their ribosomal targets, aminoglycosides can also bind, in a saturable fashion and with high affinity, to a variety of RNA structures containing non-duplex elements, such as bulges or interior loops. For example, they have been reported to interact with the type 1 human immunodeficiency virus (HIV-1) RNAs; Revresponsive element (RRE) [461] and trans-activating region (TAR) [462, 463]. One target is the Rev-response element (RRE) in type 1 human immunodeficiency virus (HIV-1) RNA, which binds the Rev protein and plays an essential role in regulating maturation of the viral RNAs [461]. Binding by aminoglycosides inhibited the Rev-RRE interaction and thus the production of HIV-1 in tissue culture [461]. Another stem-loop structure, the trans-activation response (TAR) element in HIV-1 RNA, is also targeted by aminoglycosides [464]. The interaction between TAR and the Tat protein is crucial in the initiation of RNA transcription for viral replication, which can be inhibited by neomycin-B [465]. Besides viral RNAs, aminoglycosides can target the following intracellular RNAs; iron response element (IRE) [455], the site 1 mRNA of thymidylate synthase (TS) [466], group 1 self-splicing introns [467,

468], RNase $P$ [469] and tRNA ${ }^{P h e}[470]$. As a result of these intracellular RNA targeting capabilities, aminoglycosides have been successfully used as therapeutic agents for several decades. 
TABLES AND FIGURES

Table 1. Comparison of aptamers and antibodies.

\begin{tabular}{lll}
\hline & \multicolumn{1}{c}{ Aptamers } & \multicolumn{1}{c}{ Antibodies } \\
\hline Targets & From ions to cells & Haptens or macromolecules \\
Molecular Weight & $5-25 \mathrm{kDa}$ & $150 \mathrm{kDa}$ \\
Affinity & pM to $\mu \mathrm{M}$ & pM to $\mathrm{nM}$ \\
Specificity & Distinguish a single & Distinguish a single chemical \\
& chemical group and & group and conformation \\
& conformational differences & difference \\
Immunogenicity & No & Yes \\
Tissue penetration & Moderate ability to enter & Difficult to penetrate into tissues \\
& into cells and tissues & and cells \\
Function in cells & Yes & No \\
Antidotes & Antisense oligonucleotides & No \\
Breaking the interface & Capable of blocking & Capable of blocking \\
between & macromolecule & macromolecule interactions \\
macromolecules & interactions & \\
Screening/Selection & Combinatorial chemistry & Selection in biological systems \\
process & selection (in vitro) & (in vivo) \\
Chemical modifications & Various and convenient & Limited and not convenient \\
Thermostability & Stable & Temperature sensitive \\
Shelf life & Unlimited & $\sim 5$ years \\
Active half life & Hours to days & Hours to days \\
Cost & Relatively expensive & Very expensive \\
\hline
\end{tabular}


Table 2. Metabolite binding riboswitches.

\begin{tabular}{|c|c|c|c|c|}
\hline Riboswitch & Ligand & Organism & PDB ID & References \\
\hline \multicolumn{5}{|l|}{ Amino Acid Class } \\
\hline Glycine & Glycine & Vibrio cholerae & $30 w W, 30 \times 0$ & [471] \\
\hline Lysine & Lysine & Thermotoga maritima & 3D0U, 3D0X & [472] \\
\hline S-adenosylhomocysteine & $\mathrm{SAH}$ & Ralstonia solanacearum & $3 N P Q$ & [473] \\
\hline \multicolumn{5}{|l|}{ Purine Class } \\
\hline Guanine & Hypoxanthine & Bacillus subtilis & 1U8D & [474] \\
\hline Guanine & Guanine & B. subtilis & $1 Y 27$ & [475] \\
\hline Adenine & Adenine & Vibrio vulnificus & $1 Y 26$ & [476] \\
\hline PreQ1 & PreQ0, PreQ1 & $\begin{array}{l}\text { Thermoanaerobacter } \\
\text { tengcongensis }\end{array}$ & $3 Q 50,3 Q 51$ & {$[477]$} \\
\hline PreQ1 & PreQ1 & B. subtilis & 3FU2 & {$[478]$} \\
\hline c-di-GMP & c-di-GMP & V. cholerae & $3 \mathrm{MXH}$ & [479] \\
\hline \multicolumn{5}{|l|}{ SAM-I } \\
\hline SAM-I & SAM & T. tengcongensis & $2 \mathrm{GIS}$ & [480] \\
\hline SAM-I (A94G) & & T. tengcongensis & $3 I Q P$ & [481] \\
\hline SAM-I & $\overline{S A M}$ & B. subtilis & $3 N P B$ & [482] \\
\hline SAM-II & SAM & $\begin{array}{l}\text { Sargasso Sea } \\
\text { metagenome }\end{array}$ & $2 Q W Y$ & [483] \\
\hline SAM-III & SAM & Enterococcus faecalis & $3 \mathrm{E} 5 \mathrm{C}$ & [484] \\
\hline \multicolumn{5}{|l|}{ Cofactors } \\
\hline FMN & FMN & Fusobacterium nucleatum & $3 F 2 Q$ & [485] \\
\hline TPP & TPP & Arabidopsis thaliana & $2 \mathrm{CKY}$ & [486] \\
\hline TPP & TPP & Escherichia coli & 2GDI & \\
\hline \multicolumn{5}{|l|}{ Small Molecules } \\
\hline glms & GlcN6P & T. tengcongensis & $2 Z 75$ & [487] \\
\hline M-box & $M g(I I)$ & B. subtilis & 2QBZ & [488] \\
\hline \multicolumn{5}{|l|}{ Engineered in vitro } \\
\hline Neomycin-B & Ribostamycin & Saccaromyces cerevisiea & $2 \mathrm{KXM}$ & [489] \\
\hline Tetracycline & Tetracycline & S. cerevisiae & & \\
\hline Theophylline & Theophyliine & & & \\
\hline Hoechst & Hoechst-33342 & E. coli & & \\
\hline Malachite green (MG) & Tetramethylrosamine & S. cerevisiae & & \\
\hline
\end{tabular}


Figure 1. Predicted Secondary Structures of the studied Small RNAs.
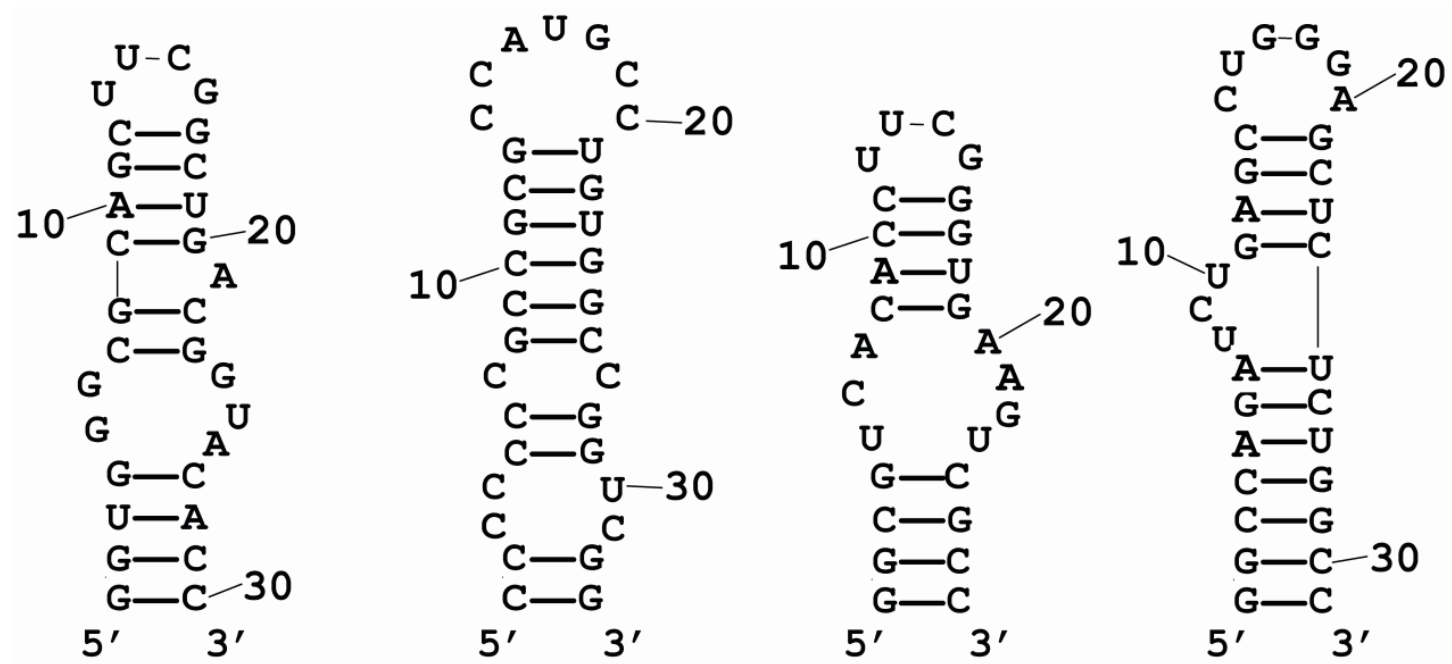

Rev-response
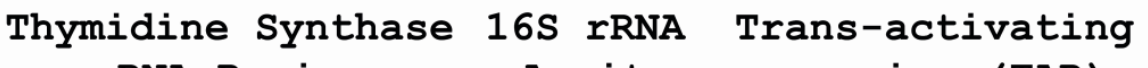

Element (RRE)

mRNA Region

A-site

region (TAR)

A G

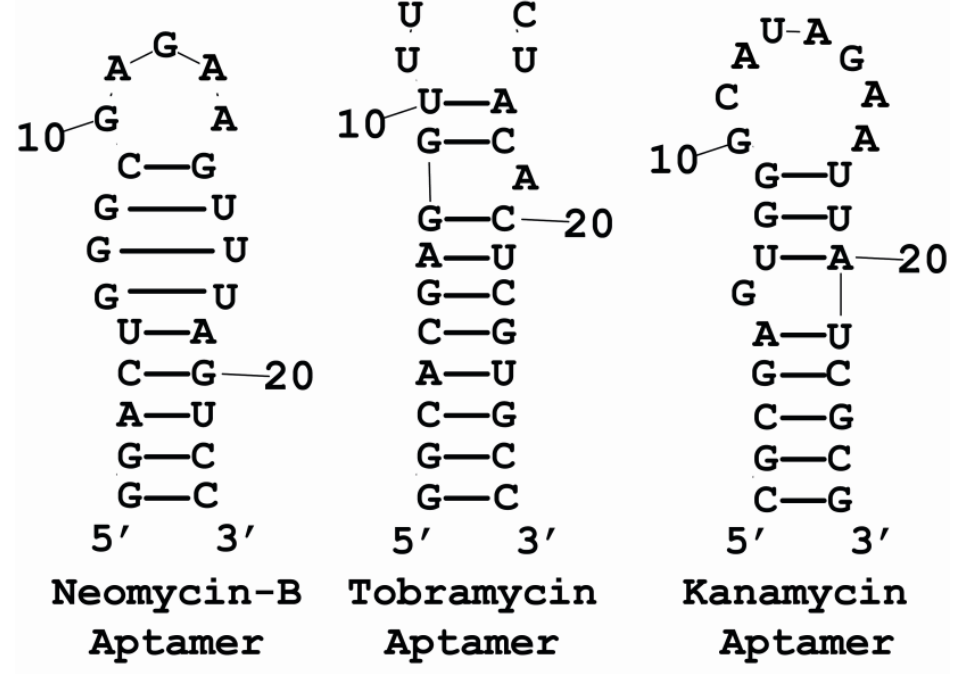

Secondary structures of the RNAs that have been shown to interact tightly with aminoglycoside antibiotics are summarized here. The RNAs shown in the top row are the RNA motifs found in nature whereas the bottom row shows the in vitro selected RNAs. The secondary structures of these small RNAs were predicted using RNAStructure 4.6 software and were then re-constructed in Adobe Illustrator ${ }^{\circledR}$. 
Figure 2. Type of possible enzymatic modification of aminoglycoside antibiotics.

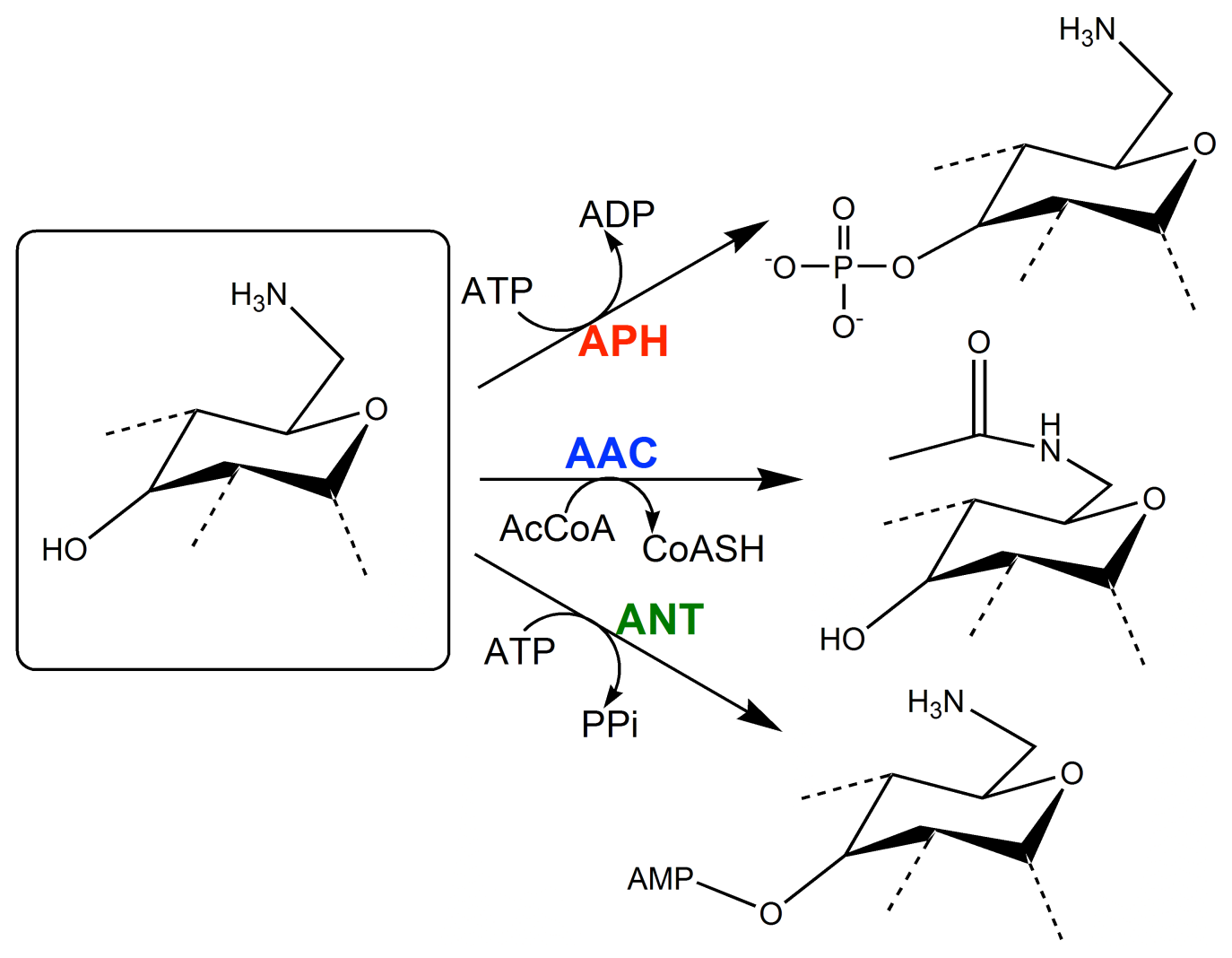


Figure 3. Sites of enzymatic modifications on kanamycin-B by various aminoglycoside-modifying enzymes.

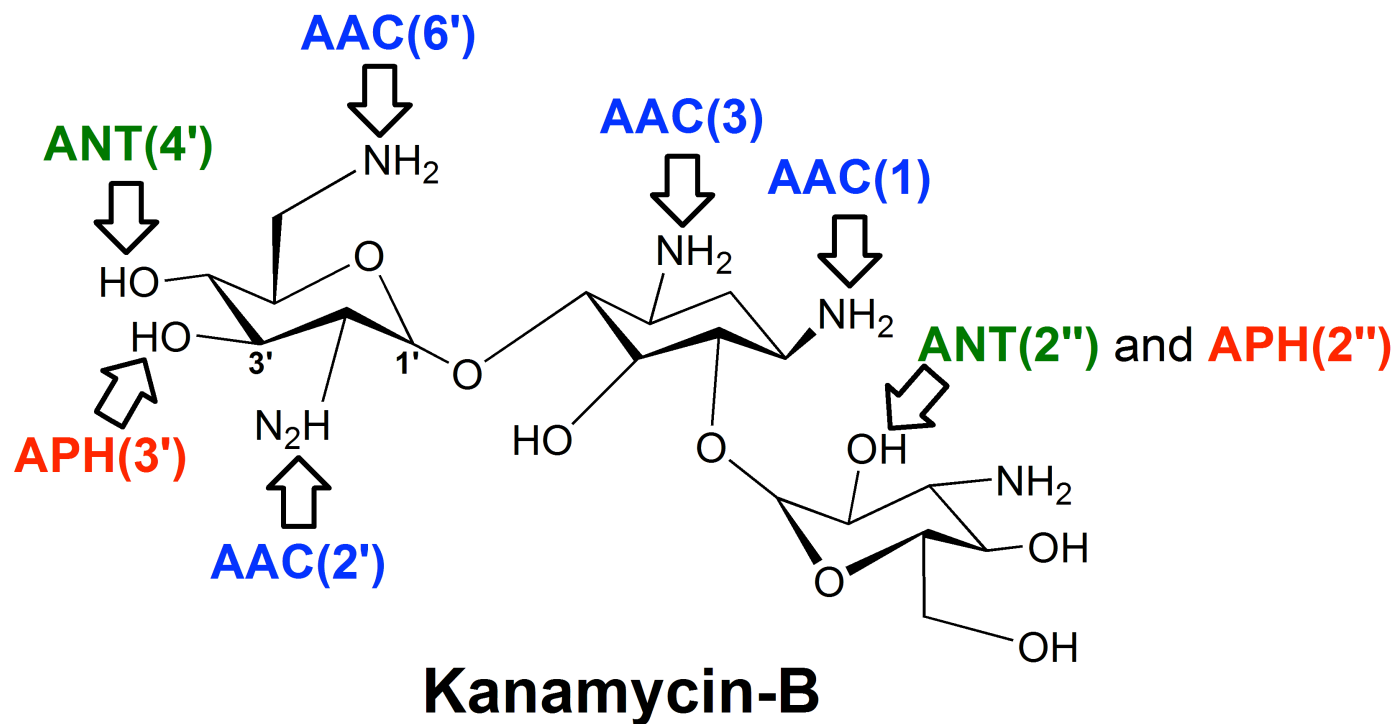


Figure 4. Chemical structures of the neomycin- and kanamycin-class aminoglycosides that interfere with the decoding process and with ribozyme catalysis.

A

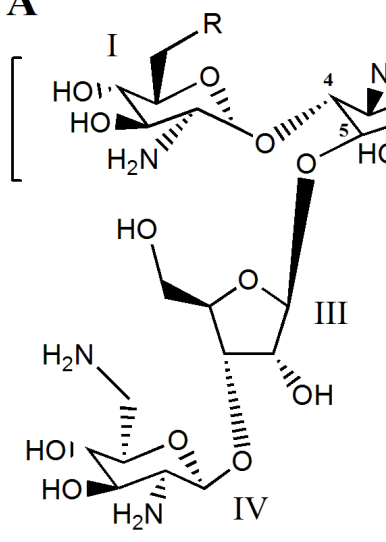

B

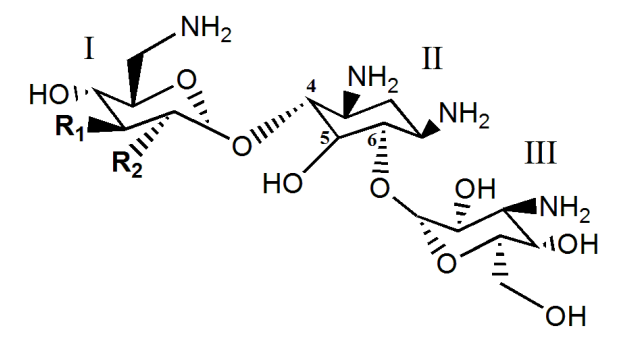

$\begin{array}{lllll} & \underline{\mathrm{R}} & \text { Kanamycin-A } & -\mathrm{OH} & -\mathrm{OH} \\ \text { Paromomycin } & -\underline{\mathrm{OH}} & \text { Kanamycin-B } & -\mathrm{OH} & -\mathrm{NH}_{2} \\ \text { Neomycin } & -\mathrm{NH}_{2} & \text { Tobramycin } & -\mathrm{H} & -\mathrm{NH}_{2}\end{array}$

Aminoglycoside antibiotics share similar chemical groups. A) Neomycin-class antibiotics have a 2-deoxystreptamine (ring II), disubstituted at positions 4 (ring I) and 5 (rings III and IV) and B) kanamycin-class antibiotics have a 2-deoxystreptamine (ring II), disubstituted at positions 4 (ring I) and 6 (rings III and IV). 


\section{REFERENCES}

1. Gesteland, R.F., T. Cech, and J.F. Atkins, The RNA world : the nature of modern RNA suggests a prebiotic RNA. 3rd ed. Cold Spring Harbor monograph series ; 43. 2006, Cold Spring Harbor, NY: Cold Spring Harbor Laboratory Press. xxiii, 768 p.

2. Kruger, K., Self-splicing RNA: autoexcision and autocyclization of the ribosomal RNA intervening sequence of tetrahymena. Cell, 1982. 31: p. 147-157.

3. Guerrier-Takada, C., et al., The RNA moiety of ribonuclease $P$ is the catalytic subunit of the enzyme. Cell, 1983. 35(3 Pt 2): p. 849-57.

4. Levine, E. and T. Hwa, Small RNAs establish gene expression thresholds. Curr Opin Microbiol, 2008. 11(6): p. 574-9.

5. Tuerk, C. and L. Gold, Systematic evolution of ligands by exponential enrichment: RNA ligands to bacteriophage T4 DNA-polymerase. Science, 1990. 249: p. 505-510.

6. Ellington, A.D. and J.W. Szostak, In vitro selection of RNA molecules that bind specific ligands. Nature, 1990. 346(6287): p. 818-22.

7. Keefe, A.D., S. Pai, and A. Ellington, Aptamers as therapeutics. Nat Rev Drug Discov, 2010. 9(7): p. 537-50.

8. Blank, M. and M. Blind, Aptamers as tools for target validation. Curr Opin Chem Biol, 2005. 9(4): p. 336-42.

9. Sefah, K., et al., Nucleic acid aptamers for biosensors and bio-analytical applications. Analyst, 2009. 134(9): p. 1765-75.

10. Nimjee, S.M., et al., The potential of aptamers as anticoagulants. Trends Cardiovasc Med, 2005. 15(1): p. 41-5.

11. Zhang, Z., M. Blank, and H.J. Schluesener, Nucleic acid aptamers in human viral disease. Arch Immunol Ther Exp (Warsz), 2004. 52(5): p. 307-15.

12. Jarosch, F., K. Buchner, and S. Klussmann, In vitro selection using a dual RNA library that allows primerless selection. Nucleic Acids Res, 2006. 34(12): p. e86.

13. Lee, J.F., et al., Aptamer database. Nucleic Acids Res, 2004. 32(Database issue): p. D95-100.

14. Jenison, R.D., et al., High-resolution molecular discrimination by RNA. Science, 1994. 263(5152): p. 1425-9.

15. Geiger, A., et al., RNA aptamers that bind L-arginine with sub-micromolar dissociation constants and high enantioselectivity. Nucleic Acids Res, 1996. 24(6): p. 1029-36. 
16. Seiwert, S.D., et al., RNA aptamers as pathway-specific MAP kinase inhibitors. Chem. Biol., 2000. 7: p. 833-843.

17. Drolet, D.W., et al., Pharmacokinetics and safety of an anti-vascular endothelial growth factor aptamer (NX1838) following injection into the vitreous humor of rhesus monkeys. Pharm Res, 2000. 17(12): p. 1503-10.

18. Teyssier, E., Temperature-sensitive mutation in yeast mitochondrial ribosome-recycling factor (RRF). Nucleic Acids Res., 2003. 31: p. 4218-4226.

19. Kaul, M. and D.S. Pilch, Thermodynamics of aminoglycoside-rRNA recognition: the binding of neomycin-class aminoglycosides to the A site of 16S rRNA. Biochemistry, 2002. 41(24): p. 7695-706.

20. Wlotzka, B., et al., In vivo properties of an anti-GnRH Spiegelmer: an example of an oligonucleotide-based therapeutic substance class. Proc Natl Acad Sci U S A, 2002. 99(13): p. 8898-902.

21. Vinores, S.A., Pegaptanib in the treatment of wet, age-related macular degeneration. Int J Nanomedicine, 2006. 1(3): p. 263-8.

22. Presta, L.G., Engineering of therapeutic antibodies to minimize immunogenicity and optimize function. Adv Drug Deliv Rev, 2006. 58(5-6): p. 640-56.

23. Griffiths, A.D., et al., Isolation of high affinity human antibodies directly from large synthetic repertoires. Embo J, 1994. 13(14): p. 3245-60.

24. Yang, X., et al., Selection of thioaptamers for diagnostics and therapeutics. Ann N Y Acad Sci, 2006. 1082: p. 116-9.

25. Baskerville, S., M. Zapp, and A.D. Ellington, Anti-Rex aptamers as mimics of the Rexbinding element. J Virol, 1999. 73(6): p. 4962-71.

26. Blind, M., W. Kolanus, and M. Famulok, Cytoplasmic RNA modulators of an inside-out signal-transduction cascade. Proc Natl Acad Sci U S A, 1999. 96(7): p. 3606-10.

27. Werstuck, G. and M.R. Green, Controlling gene expression in living cells through small molecule-RNA interactions. Science, 1998. 282(5387): p. 296-8.

28. Asahara, T., et al., VEGF contributes to postnatal neovascularization by mobilizing bone marrow-derived endothelial progenitor cells. Embo J, 1999. 18(14): p. 3964-72.

29. Peng, L., et al., A combined atomic force/fluorescence microscopy technique to select aptamers in a single cycle from a small pool of random oligonucleotides. Microsc Res Tech, 2007. 70(4): p. 372-81. 
30. Sassanfar, M. and J.W. Szostak, An RNA motif that binds ATP. Nature, 1993. 364(6437): p. 550-3.

31. Joyce, G.F., Forty years of in vitro evolution. Angew Chem Int Ed Engl, 2007. 46(34): p. 6420-36.

32. Lato, S.M., A.R. Boles, and A.D. Ellington, In vitro selection of RNA lectins: using combinatorial chemistry to interpret ribozyme evolution. Chem Biol, 1995. 2: p. 291303.

33. Kwon, M., et al., In vitro selection of RNA against kanamycin B. Mol Cells, 2001. 11(3): p. 303-11.

34. Cowan, J.A., et al., Recognition of a cognate RNA aptamer by neomycin B: quantitative evaluation of hydrogen bonding and electrostatic interactions. Nucleic Acids Res, 2000. 28(15): p. 2935-42.

35. Griffin, L.C., et al., In vivo anticoagulant properties of a novel nucleotide-based thrombin inhibitor and demonstration of regional anticoagulation in extracorporeal circuits. Blood, 1993. 81(12): p. 3271-6.

36. Richardson, F.C., et al., Quantification of 2'-fluoro-2'-deoxyuridine and 2'-fluoro-2'deoxycytidine in DNA and RNA isolated from rats and woodchucks using LC/MS/MS. Chem Res Toxicol, 2002. 15(7): p. 922-6.

37. Pieken, W.A., et al., Kinetic characterization of ribonuclease-resistant 2'-modified hammerhead ribozymes. Science, 1991. 253(5017): p. 314-7.

38. Burmeister, P.E., et al., Direct in vitro selection of a 2'-O-methyl aptamer to VEGF. Chem Biol, 2005. 12(1): p. 25-33.

39. Pagratis, N.C., et al., Potent 2'-amino-, and 2'-fluoro-2'-deoxyribonucleotide RNA inhibitors of keratinocyte growth factor. Nat Biotechnol, 1997. 15(1): p. 68-73.

40. Lin, Y. and S.D. Jayasena, Inhibition of multiple thermostable DNA polymerases by a heterodimeric aptamer. J Mol Biol, 1997. 271(1): p. 100-11.

41. White, R.R., et al., Inhibition of rat corneal angiogenesis by a nuclease-resistant RNA aptamer specific for angiopoietin-2. Proc Natl Acad Sci U S A, 2003. 100(9): p. 502833.

42. Schmidt, K.S., et al., Application of locked nucleic acids to improve aptamer in vivo stability and targeting function. Nucleic Acids Res, 2004. 32(19): p. 5757-65.

43. Eulberg, D. and S. Klussmann, Spiegelmers: biostable aptamers. Chembiochem, 2003. 4(10): p. 979-83. 
44. Nolte, A., et al., Mirror-design of L-oligonucleotide ligands binding to L-arginine. Nat Biotechnol, 1996. 14(9): p. 1116-9.

45. Williams, K.P., et al., Bioactive and nuclease-resistant L-DNA ligand of vasopressin. Proc Natl Acad Sci U S A, 1997. 94(21): p. 11285-90.

46. Kato, Y., et al., New NTP analogs: the synthesis of 4'-thioUTP and 4'-thioCTP and their utility for SELEX. Nucleic Acids Res, 2005. 33(9): p. 2942-51.

47. King, D.J., et al., Novel combinatorial selection of phosphorothioate oligonucleotide aptamers. Biochemistry, 1998. 37(47): p. 16489-93.

48. Floege, J., et al., Novel approach to specific growth factor inhibition in vivo: antagonism of platelet-derived growth factor in glomerulonephritis by aptamers. Am J Pathol, 1999. 154(1): p. 169-79.

49. Green, L.S., et al., Nuclease-resistant nucleic acid ligands to vascular permeability factor/vascular endothelial growth factor. Chem Biol, 1995. 2(10): p. 683-95.

50. Di Giusto, D.A., et al., Multitasking by multivalent circular DNA aptamers. Chembiochem, 2006. 7(3): p. 535-44.

51. Umekage, S. and Y. Kikuchi, Production of circular form of streptavidin RNA aptamer in vitro. Nucleic Acids Symp Ser (Oxf), 2006(50): p. 323-4.

52. Umekage, S. and Y. Kikuchi, Production of circular streptavidin RNA aptamer in vivo. Nucleic Acids Symp Ser (Oxf), 2007(51): p. 391-2.

53. Brody, E.N. and L. Gold, Aptamers as therapeutic and diagnostic agents. J Biotechnol, 2000. 74(1): p. 5-13.

54. Clark, S.L. and V.T. Remcho, Aptamers as analytical reagents. Electrophoresis, 2002. 23(9): p. 1335-40.

55. Burgstaller, P., A. Girod, and M. Blind, Aptamers as tools for target prioritization and lead identification. Drug Discov Today, 2002. 7(24): p. 1221-8.

56. Nimjee, S.M., C.P. Rusconi, and B.A. Sullenger, Annu. Rev. Med., 2005. 56: p. 555.

57. Tombelli, S., M. Minunni, and M. Mascini, Analytical applications of aptamers. Biosens. Bioelectron., 2005. 20: p. 2424-2434.

58. Proske, D., et al., Aptamers--basic research, drug development, and clinical applications. Appl Microbiol Biotechnol, 2005. 69(4): p. 367-74.

59. Nutiu, R. and Y. Li, Aptamers with fluorescence-signaling properties. Methods, 2005. 37(1): p. 16-25. 
60. de Franciscis, V., et al., Aptamers as innovative diagnostic and therapeutic agents in the central nervous system. CNS Neurol Disord Drug Targets, 2009. 8(5): p. 393-401.

61. Huizenga, D.E. and J.W. Szostak, A DNA aptamer that binds adenosine and ATP. Biochemistry, 1995. 34(2): p. 656-65.

62. Brackett, D.M. and T. Dieckmann, Aptamer to ribozyme: the intrinsic catalytic potential of a small RNA. Chembiochem, 2006. 7(5): p. 839-43.

63. Burgstaller, P., M. Kochoyan, and M. Famulok, Structural probing and damage selection of citrulline- and arginine-specific RNA aptamers identify base positions required for binding. Nucleic Acids Res, 1995. 23(23): p. 4769-76.

64. Vianini, E., M. Palumbo, and B. Gatto, In vitro selection of DNA aptamers that bind Ltyrosinamide. Bioorg Med Chem, 2001. 9(10): p. 2543-8.

65. Jeong, S., et al., In vitro selection of the RNA aptamer against the Sialyl Lewis $X$ and its inhibition of the cell adhesion. Biochem Biophys Res Commun, 2001. 281(1): p. 23743.

66. Wang, Y. and R.R. Rando, Specific binding of aminoglycoside antibiotics to RNA. Chem Biol, 1995. 2: p. 281-290.

67. Hamasaki, K., et al., Minimal RNA constructs that specifically bind aminoglycoside antibiotics with high affinities. Biochemistry, 1998. 37(2): p. 656-63.

68. Yang, Y., et al., Structural basis of ligand discrimination by two related RNA aptamers resolved by NMR spectroscopy. Science, 1996. 272(5266): p. 1343-7.

69. Lorsch, J.R. and J.W. Szostak, In vitro selection of RNA aptamers specific for cyanocobalamin. Biochemistry, 1994. 33(4): p. 973-82.

70. Bell, S.D., et al., RNA molecules that bind to and inhibit the active site of a tyrosine phosphatase. J Biol Chem, 1998. 273(23): p. 14309-14.

71. Kensch, O., et al., J. Biol. Chem., 2000. 275: p. 18271.

72. Lebruska, L.L. and L.J. Maher, 3rd, Selection and characterization of an RNA decoy for transcription factor NF-kappa B. Biochemistry, 1999. 38(10): p. 3168-74.

73. Fabbrizio, E., et al., Inhibition of mammalian cell proliferation by genetically selected peptide aptamers that functionally antagonize E2F activity. Oncogene, 1999. 18(30): p. 4357-63.

74. Zhai, G., et al., Characterization of RNA aptamer binding by the Wilms' tumor suppressor protein WT1. Biochemistry, 2001. 40(7): p. 2032-40. 
75. Urvil, P.T., et al., Selection of RNA aptamers that bind specifically to the NS3 protease of hepatitis C virus. Eur J Biochem, 1997. 248(1): p. 130-8.

76. Boiziau, C., et al., DNA aptamers selected against the HIV-1 trans-activation-responsive RNA element form RNA-DNA kissing complexes. J Biol Chem, 1999. 274(18): p. 12730-7.

77. Darfeuille, F., et al., RNA and N3'-->P5' kissing aptamers targeted to the trans-activation responsive (TAR) RNA of the human immunodeficiency virus-1. Nucleosides Nucleotides Nucleic Acids, 2001. 20(4-7): p. 441-9.

78. Yamamoto, R., et al., A novel RNA motif that binds efficiently and specifically to the Ttat protein of HIV and inhibits the trans-activation by Tat of transcription in vitro and in vivo. Genes Cells, 2000. 5(5): p. 371-88.

79. Sekkai, D., et al., In vitro selection of DNA aptamers against the HIV-1 TAR RNA hairpin. Antisense Nucleic Acid Drug Dev, 2002. 12(4): p. 265-74.

80. Sayer, N., et al., Structural characterization of a 2'F-RNA aptamer that binds a HIV-1 SU glycoprotein, gp120. Biochem Biophys Res Commun, 2002. 293(3): p. 924-31.

81. Hicke, B.J., et al., DNA aptamers block L-selectin function in vivo. Inhibition of human lymphocyte trafficking in SCID mice. J Clin Invest, 1996. 98(12): p. 2688-92.

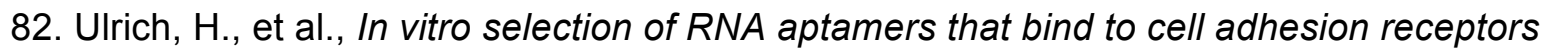
of Trypanosoma cruzi and inhibit cell invasion. J Biol Chem, 2002. 277(23): p. 20756-62.

83. Jenison, R.D., et al., Oligonucleotide inhibitors of P-selectin-dependent neutrophilplatelet adhesion. Antisense Nucleic Acid Drug Dev, 1998. 8(4): p. 265-79.

84. Jellinek, D., et al., Inhibition of receptor-binding by high-affinity RNA ligands to vascular endothelial growth-factor. Biochemistry, 1994. 33: p. 10450-10456.

85. Binkley, J., et al., RNA ligands to human nerve growth factor. Nucleic Acids Res, 1995. 23(16): p. 3198-205.

86. Bell, C., et al., Oligonucleotide NX1838 inhibits VEGF165-mediated cellular responses in vitro. In Vitro Cell Dev Biol Anim, 1999. 35(9): p. 533-42.

87. Hartmann, G., et al., Cytokines and therapeutic oligonucleotides. Cytokines Cell Mol Ther, 1997. 3(4): p. 247-56.

88. Hamaguchi, N., A. Ellington, and M. Stanton, Aptamer beacons for the direct detection of proteins. Anal Biochem, 2001. 294(2): p. 126-31. 
89. Blank, M., et al., Systematic evolution of a DNA aptamer binding to rat brain tumor microvessels. selective targeting of endothelial regulatory protein pigpen. J Biol Chem, 2001. 276(19): p. 16464-8.

90. Nutiu, R. and Y. Li, Structure-switching signaling aptamers. J Am Chem Soc, 2003. 125(16): p. 4771-8.

91. Li, W., et al., FRET-based aptamer probe for rapid angiogenin detection. Talanta, 2008. 75(3): p. 770-4.

92. Kim, D., Y.Y. Jeong, and S. Jon, A drug-loaded aptamer-gold nanoparticle bioconjugate for combined CT imaging and therapy of prostate cancer. ACS Nano, 2010. 4(7): p. 3689-96.

93. Jayasena, S.D., Clin. Chem., 1999. 45: p. 1628.

94. Murphy, M.B., et al., An improved method for the in vitro evolution of aptamers and applications in protein detection and purification. Nucleic Acids Res., 2003. 31: p. e110.

95. Dick, L.W., Jr. and L.B. McGown, Aptamer-enhanced laser desorption/ionization for affinity mass spectrometry. Anal Chem, 2004. 76(11): p. 3037-41.

96. Fredriksson, S., et al., Nat. Biotechnol., 2002. 20: p. 473.

97. Stojanovic, M.N. and D.M. Kolpashchikov, Modular aptameric sensors. J. Am. Chem. Soc., 2004. 126: p. 9266-9270.

98. Stojanovic, M.N. and D.W. Landry, Aptamer-based colorimetric probe for cocaine. J Am Chem Soc, 2002. 124(33): p. 9678-9.

99. Smith, D., et al., In vitro selection of RNA-based irreversible inhibitors of human neutrophil elastase. Chem Biol, 1995. 2(11): p. 741-50.

100. Charlton, J., G.P. Kirschenheuter, and D. Smith, Highly potent irreversible inhibitors of neutrophil elastase generated by selection from a randomized DNA-valine phosphonate library. Biochemistry, 1997. 36(10): p. 3018-26.

101. Lin, Y., et al., Peptide conjugation to an in vitro-selected DNA ligand improves enzyme inhibition. Proc Natl Acad Sci U S A, 1995. 92(24): p. 11044-8.

102. Giver, L., et al., Selection and design of high-affinity RNA ligands for HIV-1 Rev. Gene, 1993. 137(1): p. 19-24.

103. Allen, P., S. Worland, and L. Gold, Isolation of high-affinity RNA ligands to HIV-1 integrase from a random pool. Virology, 1995. 209(2): p. 327-36. 
104. Tuerk, C., S. MacDougal, and L. Gold, RNA pseudoknots that inhibit human immunodeficiency virus type 1 reverse transcriptase. Proc. Natl Acad. Sci. USA, 1992. 89: p. 6988-6992.

105. Lato, S.M., et al., Boron-containing aptamers to ATP. Nucleic Acids Res, 2002. 30(6): p. 1401-7.

106. Coughlin, S.R., Thrombin signalling and protease-activated receptors. Nature, 2000. 407(6801): p. 258-64.

107. White, W., Generation of species cross-reactive aptamers using 'toggle' SELEX. Mol. Ther., 2001. 4: p. 567-573.

108. Ng, E.W. and A.P. Adamis, Targeting angiogenesis, the underlying disorder in neovascular age-related macular degeneration. Can J Ophthalmol, 2005. 40(3): p. 352-68.

109. Ruckman, J., et al., 2'-Fluoropyrimidine RNA-based aptamers to the 165-amino acid form of vascular endothelial growth factor (VEGF165). Inhibition of receptor binding and VEGF-induced vascular permeability through interactions requiring the exon 7encoded domain. J Biol Chem, 1998. 273(32): p. 20556-67.

110. Tucker, C.E., et al., Detection and plasma pharmacokinetics of an anti-vascular endothelial growth factor oligonucleotide-aptamer (NX1838) in rhesus monkeys. J Chromatogr B Biomed Sci Appl, 1999. 732(1): p. 203-12.

111. Watson, S.R., et al., Anti-L-selectin aptamers: binding characteristics, pharmacokinetic parameters, and activity against an intravascular target in vivo. Antisense Nucleic Acid Drug Dev, 2000. 10(2): p. 63-75.

112. Burke, D.H. and J.H. Willis, Recombination, RNA evolution, and bifunctional RNA molecules isolated through chimeric SELEX. Rna, 1998. 4(9): p. 1165-75.

113. Di Giusto, D.A. and G.C. King, Construction, stability, and activity of multivalent circular anticoagulant aptamers. J Biol Chem, 2004. 279(45): p. 46483-9.

114. Elbaz, J., et al., Parallel analysis of two analytes in solutions or on surfaces by using a bifunctional aptamer: applications for biosensing and logic gate operations. Chembiochem, 2008. 9(2): p. 232-9.

115. Santulli-Marotto, S., et al., Multivalent RNA aptamers that inhibit CTLA-4 and enhance tumor immunity. Cancer Res, 2003. 63(21): p. 7483-9.

116. McNamara, J.O., et al., Multivalent 4-1BB binding aptamers costimulate CD8+ $T$ cells and inhibit tumor growth in mice. J Clin Invest, 2008. 118(1): p. 376-86. 
117. Davis, K.A., et al., Use of a high affinity DNA ligand in flow cytometry. Nucleic Acids Res, 1996. 24(4): p. 702-6.

118. Babendure, J.R., S.R. Adams, and R.Y. Tsien, Aptamers switch on fluorescence of triphenylmethane dyes. J Am Chem Soc, 2003. 125(48): p. 14716-7.

119. Fusco, D., et al., Single mRNA molecules demonstrate probabilistic movement in living mammalian cells. Curr Biol, 2003. 13(2): p. 161-7.

120. Tucker, B.J. and R.R. Breaker, Riboswitches as versatile gene control elements. Curr Opin Struct Biol, 2005. 15(3): p. 342-8.

121. Grate, D. and C. Wilson, Laser-mediated, site-specific inactivation of RNA transcripts. Proc Natl Acad Sci U S A, 1999. 96(11): p. 6131-6.

122. Grate, D. and C. Wilson, Inducible regulation of the S. cerevisiae cell cycle mediated by an RNA aptamer-ligand complex. Bioorg Med Chem, 2001. 9(10): p. 2565-70.

123. Buskirk, A.R., A. Landrigan, and D.R. Liu, Engineering a ligand-dependent RNA transcriptional activator. Chem Biol, 2004. 11(8): p. 1157-63.

124. Wang, S., et al., Knocking down gene function with an RNA aptamer expressed as part of an intron. Nucleic Acids Res, 2010. 38(15): p. e154.

125. Burke, D.H. and D.G. Nickens, Expressing RNA aptamers inside cells to reveal proteome and ribonome function. Brief Funct Genomic Proteomic, 2002. 1(2): p. 16988.

126. Jain, C. and J.G. Belasco, Rapid genetic analysis of RNA-protein interactions by translational repression in Escherichia coli. Methods Enzymol, 2000. 318: p. 309-32.

127. Nickens, D.G., J.T. Patterson, and D.H. Burke, Inhibition of HIV-1 reverse transcriptase by RNA aptamers in Escherichia coli. RNA, 2003. 9: p. 1029-1033.

128. Chaloin, L., et al., Endogenous expression of a high-affinity pseudoknot RNA aptamer suppresses replication of HIV-1. Nucleic Acid Res., 2002. 30: p. 4001-4008.

129. Joshi, P.J., T.W. North, and V.R. Prasad, Aptamers directed to HIV-1 reverse transcriptase display greater efficacy over small hairpin RNAs targeted to viral RNA in blocking HIV-1 replication. Mol Ther, 2005. 11(5): p. 677-86.

130. Yang, C., et al., RNA aptamers targeting the cell death inhibitor CED-9 induce cell killing in Caenorhabditis elegans. J Biol Chem, 2006. 281(14): p. 9137-44.

131. Jian, Y., et al., RNA aptamers interfering with nucleophosmin oligomerization induce apoptosis of cancer cells. Oncogene, 2009. 28(47): p. 4201-11. 
132. Dahlberg, A.E., The functional role of ribosomal RNA in protein synthesis. Cell, 1989. 57(4): p. 525-9.

133. Pfister, P., et al., The molecular basis for A-site mutations conferring aminoglycoside resistance: relationship between ribosomal susceptibility and X-ray crystal structures. Chembiochem, 2003. 4(10): p. 1078-88.

134. Bailor, M.H., X. Sun, and H.M. Al-Hashimi, Topology links RNA secondary structure with global conformation, dynamics, and adaptation. Science, 2010. 327(5962): p. 202-6.

135. Auffinger, P., S. Louise-May, and E. Westhof, Molecular dynamics simulations of solvated yeast tRNA(Asp). Biophys J, 1999. 76(1 Pt 1): p. 50-64.

136. Schneider, C., M. Brandl, and J. Suhnel, Molecular dynamics simulation reveals conformational switching of water-mediated uracil-cytosine base-pairs in an RNA duplex. J Mol Biol, 2001. 305(4): p. 659-67.

137. Correll, C.C., I.G. Wool, and A. Munishkin, The two faces of the Escherichia coli 23 S rRNA sarcin/ricin domain: the structure at 1.11 A resolution. J Mol Biol, 1999. 292(2): p. $275-87$.

138. Muriaux, D., P. Fosse, and J. Paoletti, $A$ kissing complex together with a stable dimer is involved in the HIV-1Lai RNA dimerization process in vitro. Biochemistry, 1996. 35(15): p. 5075-82.

139. Reblova, K., et al., Molecular dynamics simulations of RNA kissing-loop motifs reveal structural dynamics and formation of cation-binding pockets. Nucleic Acids Res, 2003. 31(23): p. 6942-52.

140. Razga, F., et al., Hinge-like motions in RNA kink-turns: the role of the second a-minor motif and nominally unpaired bases. Biophys J, 2005. 88(5): p. 3466-85.

141. Gilbert, W., The RNA world. Nature, 1986. 319: p. 818.

142. Krasovska, M.V., et al., Structural dynamics of precursor and product of the RNA enzyme from the hepatitis delta virus as revealed by molecular dynamics simulations. J Mol Biol, 2005. 351(4): p. 731-48.

143. Sefcikova, J., et al., The genomic HDV ribozyme utilizes a previously unnoticed U-turn motif to accomplish fast site-specific catalysis. Nucleic Acids Res, 2007. 35(6): p. 1933-46. 
144. Wang, T., et al., Computational and experimental analyses converge to reveal a coherent yet malleable aptamer structure that controls chemical reactivity. J Am Chem Soc, 2009. 131(41): p. 14747-55.

145. Stelzer, A.C., et al., Constructing atomic-resolution RNA structural ensembles using MD and motionally decoupled NMR RDCs. Methods, 2009. 49(2): p. 167-73.

146. Grundy, F.J. and T.M. Henkin, The $S$ box regulon: a new global transcription termination control system for methionine and cysteine biosynthesis genes in grampositive bacteria. Mol. Microbiol., 1998. 30(4): p. 737-49.

147. Yarnell, W.S. and J.W. Roberts, Mechanism of intrinsic transcription termination and antitermination. Science, 1999. 284(5414): p. 611-5.

148. Waters, L.S. and G. Storz, Regulatory RNAs in bacteria. Cell, 2009. 136(4): p. 615-28.

149. Mandal, M. and R.R. Breaker, Gene regulation by riboswitches. Nature Rev. Mol. Cell Biol., 2004. 5: p. 451-463.

150. Henkin, T.M., Riboswitch RNAs: using RNA to sense cellular metabolism. Genes Dev, 2008. 22(24): p. 3383-90.

151. Boehr, D.D., R. Nussinov, and P.E. Wright, The role of dynamic conformational ensembles in biomolecular recognition. Nat Chem Biol, 2009. 5(11): p. 789-96.

152. Winkler, W.C. and R.R. Breaker, Genetic control by metabolite-binding riboswitches. ChemBioChem, 2003. 4: p. 1024-1032.

153. Grundy, F.J., S.C. Lehman, and T.M. Henkin, The $L$ box regulon: lysine sensing by leader RNAs of bacterial lysine biosynthesis genes. Proc Natl Acad Sci U S A, 2003. 100(21): p. 12057-62.

154. Mandal, M., et al., A glycine-dependent riboswitch that uses cooperative binding to control gene expression. Science, 2004. 306(5694): p. 275-9.

155. Fuchs, R.T., F.J. Grundy, and T.M. Henkin, S-adenosylmethionine directly inhibits binding of 30 S ribosomal subunits to the SMK box translational riboswitch RNA. Proc Natl Acad Sci U S A, 2007. 104(12): p. 4876-80.

156. Winkler, W.C., et al., An mRNA structure that controls gene expression by binding Sadenosylmethionine. Nat Struct Biol, 2003. 10(9): p. 701-7.

157. Epshtein, V., A.S. Mironov, and E. Nudler, The riboswitch-mediated control of sulfur metabolism in bacteria. Proc Natl Acad Sci U S A, 2003. 100(9): p. 5052-6.

158. Wang, J.X., et al., Riboswitches that sense S-adenosylhomocysteine and activate genes involved in coenzyme recycling. Mol Cell, 2008. 29(6): p. 691-702. 
159. Mironov, A.S., et al., Sensing small molecules by nascent RNA: a mechanism to control transcription in bacteria. Cell, 2002. 111(5): p. 747-56.

160. Nahvi, A., et al., Genetic control by a metabolite binding mRNA. Chem Biol, 2002. 9(9): p. 1043.

161. Winkler, W.C., et al., Control of gene expression by a natural metabolite-responsive ribozyme. Nature, 2004. 428: p. 281-286.

162. Barrick, J.E. and R.R. Breaker, The distributions, mechanisms, and structures of metabolite-binding riboswitches. Genome Biol, 2007. 8(11): p. R239.

163. Sudarsan, N., et al., Riboswitches in eubacteria sense the second messenger cyclic diGMP. Science, 2008. 321(5887): p. 411-3.

164. Spinelli, S.V., et al., Regulation of magnesium homeostasis in Salmonella: $M g(2+)$ targets the mgtA transcript for degradation by RNase E. FEMS Microbiol Lett, 2008. 280(2): p. 226-34.

165. Regulski, E.E., et al., A widespread riboswitch candidate that controls bacterial genes involved in molybdenum cofactor and tungsten cofactor metabolism. Mol Microbiol, 2008. 68(4): p. 918-32.

166. Morita, M.T., et al., Translational induction of heat shock transcription factor sigma32: evidence for a built-in RNA thermosensor. Genes Dev, 1999. 13(6): p. 655-65.

167. Nechooshtan, G., et al., A pH-responsive riboregulator. Genes Dev, 2009. 23(22): p. 2650-62.

168. Collins, J.A., et al., Mechanism of mRNA destabilization by the glmS ribozyme. Genes Dev, 2007. 21(24): p. 3356-68.

169. Wachter, A., et al., Riboswitch control of gene expression in plants by splicing and alternative 3' end processing of mRNAs. Plant Cell, 2007. 19(11): p. 3437-50.

170. Bocobza, S., et al., Riboswitch-dependent gene regulation and its evolution in the plant kingdom. Genes Dev, 2007. 21(22): p. 2874-9.

171. Cheah, M.T., et al., Control of alternative RNA splicing and gene expression by eukaryotic riboswitches. Nature, 2007. 447(7143): p. 497-500.

172. Mandal, M. and R.R. Breaker, Adenine riboswitches and gene activation by disruption of a transcription terminator. Nat Struct Mol Biol, 2004. 11(1): p. 29-35.

173. Gelfand, M.S., et al., A conserved RNA structure element involved in the regulation of bacterial riboflavin synthesis genes. Trends Genet, 1999. 15(11): p. 439-42. 
174. Miranda-Rios, J., M. Navarro, and M. Soberon, A conserved RNA structure (thi box) is involved in regulation of thiamin biosynthetic gene expression in bacteria. Proc Natl Acad Sci U S A, 2001. 98(17): p. 9736-41.

175. Corbino, K.A., et al., Evidence for a second class of S-adenosylmethionine riboswitches and other regulatory RNA motifs in alpha-proteobacteria. Genome Biol, 2005. 6(8): p. R70.

176. Fuchs, R.T., F.J. Grundy, and T.M. Henkin, The $S(M K)$ box is a new SAM-binding RNA for translational regulation of SAM synthetase. Nat Struct Mol Biol, 2006. 13(3): p. 226-33.

177. Yousef, M.R., F.J. Grundy, and T.M. Henkin, Structural transitions induced by the interaction between $\operatorname{RNA}(\mathrm{Gly})$ and the Bacillus subtilis glyQS T box leader RNA. J Mol Biol, 2005. 349(2): p. 273-87.

178. Yen, L., Exogenous control of mammalian gene expression through modulation of RNA self-cleavage. Nature, 2004. 431: p. 471-476.

179. Yen, L., et al., Identification of inhibitors of ribozyme self-cleavage in mammalian cells via high-throughput screening of chemical libraries. RNA, 2006. 12(5): p. 797-806.

180. Isaacs, F.J., et al., Engineered riboregulators enable post-transcriptional control of gene expression. Nat Biotechnol, 2004. 22(7): p. 841-7.

181. Bayer, T.S. and C.D. Smolke, Programmable ligand-controlled riboregulators of eukaryotic gene expression. Nat Biotechnol, 2005. 23(3): p. 337-43.

182. Suess, B., Conditional gene expression by controlling translation with tetracyclinebinding aptamers. Nucleic Acids Res., 2003. 31: p. 1853-1858.

183. Weigand, J.E., et al., Screening for engineered neomycin riboswitches that control translation initiation. RNA, 2008. 14(1): p. 89-97.

184. Hanson, S., et al., Tetracycline-aptamer-mediated translational regulation in yeast. Mol Microbiol, 2003. 49(6): p. 1627-37.

185. Harvey, A.J. and M.R. Crompton, Use of RNA interference to validate Brk as a novel therapeutic target in breast cancer: Brk promotes breast carcinoma cell proliferation. Oncogene, 2003. 22(32): p. 5006-10.

186. Fourmy, D., et al., Structure of the A site of Escherichia coli 165 ribosomal RNA complexed with an aminoglycoside antibiotic. Science, 1996. 274(5291): p. 1367-71. 187. Wallis, M.G., et al., A novel RNA motif for neomycin recognition. Chem Biol, 1995. 2: p. 543-552. 
188. Hammond, M.C., RNA folding: a tale of two riboswitches. Nat Chem Biol, 2011. 7(6): p. 342-3.

189. Tsien, R.Y., Imagining imaging's future. Nat Rev Mol Cell Biol, 2003. Suppl: p. SS16-21.

190. Rosenthal, M.S., et al., Quantitative SPECT imaging: a review and recommendations by the Focus Committee of the Society of Nuclear Medicine Computer and Instrumentation Council. J Nucl Med, 1995. 36(8): p. 1489-513.

191. Gambhir, S.S., Molecular imaging of cancer with positron emission tomography. Nat Rev Cancer, 2002. 2(9): p. 683-93.

192. Ozker, K., Current developments in single photon radiopharmaceuticals for tumor imaging. Curr Pharm Des, 2000. 6(11): p. 1123-6.

193. Wu, J.C., et al., Molecular imaging of cardiac cell transplantation in living animals using optical bioluminescence and positron emission tomography. Circulation, 2003. 108(11): p. 1302-5.

194. Alam, J. and J.L. Cook, Reporter genes: application to the study of mammalian gene transcription. Anal Biochem, 1990. 188(2): p. 245-54.

195. de Wet, J.R., et al., Firefly luciferase gene: structure and expression in mammalian cells. Mol Cell Biol, 1987. 7(2): p. 725-37.

196. Contag, C.H. and M.H. Bachmann, Advances in in vivo bioluminescence imaging of gene expression. Annu Rev Biomed Eng, 2002. 4: p. 235-60.

197. Mempel, T.R., et al., In vivo imaging of leukocyte trafficking in blood vessels and tissues. Curr Opin Immunol, 2004. 16(4): p. 406-17.

198. Shimomura, O., F.H. Johnson, and Y. Saiga, Extraction, purification and properties of aequorin, a bioluminescent protein from the luminous hydromedusan, Aequorea. J Cell Comp Physiol, 1962. 59: p. 223-39.

199. Morise, H., et al., Intermolecular energy transfer in the bioluminescent system of Aequorea. Biochemistry, 1974. 13(12): p. 2656-62.

200. Cody, C.W., et al., Chemical structure of the hexapeptide chromophore of the Aequorea green-fluorescent protein. Biochemistry, 1993. 32(5): p. 1212-8.

201. Heim, R., D.C. Prasher, and R.Y. Tsien, Wavelength mutations and posttranslational autoxidation of green fluorescent protein. Proc Natl Acad Sci U S A, 1994. 91(26): p. 12501-4.

202. Chalfie, M., et al., Green fluorescent protein as a marker for gene expression. Science, 1994. 263(5148): p. 802-5. 
203. Cormack, B.P., R.H. Valdivia, and S. Falkow, FACS-optimized mutants of the green fluorescent protein (GFP). Gene, 1996. 173(1 Spec No): p. 33-8.

204. Yang, T.T., L. Cheng, and S.R. Kain, Optimized codon usage and chromophore mutations provide enhanced sensitivity with the green fluorescent protein. Nucleic Acids Res, 1996. 24(22): p. 4592-3.

205. Zhang, G., V. Gurtu, and S.R. Kain, An enhanced green fluorescent protein allows sensitive detection of gene transfer in mammalian cells. Biochem Biophys Res Commun, 1996. 227(3): p. 707-11.

206. Heim, R. and R.Y. Tsien, Engineering green fluorescent protein for improved brightness, longer wavelengths and fluorescence resonance energy transfer. Curr Biol, 1996. 6(2): p. 178-82.

207. Miyawaki, A., et al., Fluorescent indicators for Ca2+ based on green fluorescent proteins and calmodulin. Nature, 1997. 388(6645): p. 882-7.

208. Nagai, T., et al., $A$ variant of yellow fluorescent protein with fast and efficient maturation for cell-biological applications. Nat Biotechnol, 2002. 20(1): p. 87-90.

209. Griesbeck, O., et al., Reducing the environmental sensitivity of yellow fluorescent protein. Mechanism and applications. J Biol Chem, 2001. 276(31): p. 29188-94.

210. Rizzo, M.A., et al., An improved cyan fluorescent protein variant useful for FRET. Nat Biotechnol, 2004. 22(4): p. 445-9.

211. Mena, M.A., et al., Blue fluorescent proteins with enhanced brightness and photostability from a structurally targeted library. Nat Biotechnol, 2006. 24(12): p. 1569-71.

212. Matz, M.V., et al., Fluorescent proteins from nonbioluminescent Anthozoa species. Nat Biotechnol, 1999. 17(10): p. 969-73.

213. Bevis, B.J. and B.S. Glick, Rapidly maturing variants of the Discosoma red fluorescent protein (DsRed). Nat Biotechnol, 2002. 20(1): p. 83-7.

214. Campbell, R.E., et al., A monomeric red fluorescent protein. Proc Natl Acad Sci U S A, 2002. 99(12): p. 7877-82.

215. Shaner, N.C., et al., Improved monomeric red, orange and yellow fluorescent proteins derived from Discosoma sp. red fluorescent protein. Nat Biotechnol, 2004. 22(12): p. 1567-72.

216. Wang, L., et al., Evolution of new nonantibody proteins via iterative somatic hypermutation. Proc Natl Acad Sci U S A, 2004. 101(48): p. 16745-9. 
217. Chudakov, D.M., S. Lukyanov, and K.A. Lukyanov, Fluorescent proteins as a toolkit for in vivo imaging. Trends Biotechnol, 2005. 23(12): p. 605-13.

218. Shaner, N.C., P.A. Steinbach, and R.Y. Tsien, A guide to choosing fluorescent proteins. Nat Methods, 2005. 2(12): p. 905-9.

219. Shah, K., et al., Bimodal viral vectors and in vivo imaging reveal the fate of human neural stem cells in experimental glioma model. J Neurosci, 2008. 28(17): p. 4406-13.

220. Aoyagi, Y., et al., Energy cost of whole-body protein synthesis measured in vivo in chicks. Comp Biochem Physiol A, 1988. 91(4): p. 765-8.

221. Alwine, J.C., et al., Detection of specific RNAs or specific fragments of DNA by fractionation in gels and transfer to diazobenzyloxymethyl paper. Methods Enzymol, 1979. 68: p. 220-42.

222. Bassell, G.J., et al., Single mRNAs visualized by ultrastructural in situ hybridization are principally localized at actin filament intersections in fibroblasts. J Cell Biol, 1994. 126(4): p. 863-76.

223. Volpi, E.V. and J.M. Bridger, FISH glossary: an overview of the fluorescence in situ hybridization technique. Biotechniques, 2008. 45(4): p. 385-6, 388, 390 passim.

224. Femino, A.M., et al., Visualization of single RNA transcripts in situ. Science, 1998. 280(5363): p. 585-90.

225. Guzowski, J.F., et al., Imaging neural activity with temporal and cellular resolution using FISH. Curr Opin Neurobiol, 2001. 11(5): p. 579-84.

226. Molenaar, C., et al., Poly $(A)+R N A$ s roam the cell nucleus and pass through speckle domains in transcriptionally active and inactive cells. J Cell Biol, 2004. 165(2): p. 191-202.

227. Molenaar, C., et al., Linear 2' O-Methyl RNA probes for the visualization of RNA in living cells. Nucleic Acids Res, 2001. 29(17): p. E89-9.

228. Tsuji, A., et al., Direct observation of specific messenger RNA in a single living cell under a fluorescence microscope. Biophys J, 2000. 78(6): p. 3260-74.

229. Glotzer, J.B., et al., Cytoplasmic flows localize injected oskar RNA in Drosophila oocytes. Curr Biol, 1997. 7(5): p. 326-37.

230. Jacobson, M.R. and T. Pederson, Localization of signal recognition particle RNA in the nucleolus of mammalian cells. Proc Natl Acad Sci U S A, 1998. 95(14): p. 7981-6.

231. Bonnet, G., O. Krichevsky, and A. Libchaber, Kinetics of conformational fluctuations in DNA hairpin-loops. Proc Natl Acad Sci U S A, 1998. 95(15): p. 8602-6. 
232. Bonnet, G., et al., Thermodynamic basis of the enhanced specificity of structured DNA probes. Proc Natl Acad Sci U S A, 1999. 96(11): p. 6171-6.

233. Roberts, R.W. and D.M. Crothers, Specificity and stringency in DNA triplex formation. Proc Natl Acad Sci U S A, 1991. 88(21): p. 9397-401.

234. Tsourkas, A., et al., Hybridization kinetics and thermodynamics of molecular beacons. Nucleic Acids Res, 2003. 31(4): p. 1319-30.

235. Tyagi, S. and F.R. Kramer, Molecular beacons: probes that fluoresce upon hybridization. Nat Biotechnol, 1996. 14(3): p. 303-8.

236. Leonetti, J.P., et al., Intracellular distribution of microinjected antisense oligonucleotides. Proc Natl Acad Sci U S A, 1991. 88(7): p. 2702-6.

237. Mechti, N., et al., Nuclear location of synthetic oligonucleotides microinjected somatic cells: its implication in an antisense strategy. Nucleic Acids Symp Ser, 1991(24): p. 147-50.

238. Nitin, N., et al., Peptide-linked molecular beacons for efficient delivery and rapid mRNA detection in living cells. Nucleic Acids Res, 2004. 32(6): p. e58.

239. Tyagi, S. and O. Alsmadi, Imaging native beta-actin mRNA in motile fibroblasts. Biophys J, 2004. 87(6): p. 4153-62.

240. Santangelo, P.J., Molecular beacons and related probes for intracellular RNA imaging. Wiley Interdiscip Rev Nanomed Nanobiotechnol, 2010. 2(1): p. 11-9.

241. Wansink, D.G., et al., Fluorescent labeling of nascent RNA reveals transcription by RNA polymerase II in domains scattered throughout the nucleus. J Cell Biol, 1993. 122(2): p. 283-93.

242. Bertrand, E., et al., Localization of ASH1 mRNA particles in living yeast. Mol Cell, 1998. 2(4): p. 437-45.

243. Forrest, K.M. and E.R. Gavis, Live imaging of endogenous RNA reveals a diffusion and entrapment mechanism for nanos mRNA localization in Drosophila. Curr Biol, 2003. 13(14): p. 1159-68.

244. Haim, L., et al., A genomic integration method to visualize localization of endogenous $m R N A s$ in living yeast. Nat Methods, 2007. 4(5): p. 409-12.

245. Magliery, T.J., et al., Detecting protein-protein interactions with a green fluorescent protein fragment reassembly trap: scope and mechanism. J Am Chem Soc, 2005. 127(1): p. 146-57. 
246. DiMasi, J.A., The value of improving the productivity of the drug development process: faster times and better decisions. Pharmacoeconomics, 2002. 20 Suppl 3: p. 1-10.

247. DiMasi, J.A., R.W. Hansen, and H.G. Grabowski, The price of innovation: new estimates of drug development costs. J Health Econ, 2003. 22(2): p. 151-85.

248. Adams, C.P. and V.V. Brantner, Estimating the cost of new drug development: is it really 802 million dollars? Health Aff (Millwood), 2006. 25(2): p. 420-8.

249. Simon, S.M. and M. Schindler, Cell biological mechanisms of multidrug resistance in tumors. Proc Natl Acad Sci U S A, 1994. 91(9): p. 3497-504.

250. Russell, A.D., Mechanisms of bacterial resistance to antibiotics and biocides. Prog Med Chem, 1998. 35: p. 133-97.

251. Neu, H.C., Penicillin-binding proteins and role of amdinocillin in causing bacterial cell death. Am J Med, 1983. 75(2A): p. 9-20.

252. Eady, E.A., et al., Distribution of genes encoding erythromycin ribosomal methylases and an erythromycin efflux pump in epidemiologically distinct groups of staphylococci. J Antimicrob Chemother, 1993. 31(2): p. 211-7.

253. Swedberg, G., S. Ringertz, and O. Skold, Sulfonamide resistance in Streptococcus pyogenes is associated with differences in the amino acid sequence of its chromosomal dihydropteroate synthase. Antimicrob Agents Chemother, 1998. 42(5): p. 1062-7.

254. Li, X.Z. and H. Nikaido, Efflux-mediated drug resistance in bacteria: an update. Drugs, 2009. 69(12): p. 1555-623.

255. Li, X.Z., L. Zhang, and H. Nikaido, Efflux pump-mediated intrinsic drug resistance in Mycobacterium smegmatis. Antimicrob Agents Chemother, 2004. 48(7): p. 2415-23.

256. Cannon, R.D., et al., Efflux-mediated antifungal drug resistance. Clin Microbiol Rev, 2009. 22(2): p. 291-321, Table of Contents.

257. Gottesman, M.M., Mechanisms of cancer drug resistance. Annu Rev Med, 2002. 53: p. 615-27.

258. Pages, J.M., M. Masi, and J. Barbe, Inhibitors of efflux pumps in Gram-negative bacteria. Trends Mol Med, 2005. 11(8): p. 382-9.

259. Poole, K., Efflux pumps as antimicrobial resistance mechanisms. Ann Med, 2007. 39(3): p. 162-76.

260. Webber, M.A. and L.J. Piddock, The importance of efflux pumps in bacterial antibiotic resistance. J Antimicrob Chemother, 2003. 51(1): p. 9-11. 
261. Rosenberg, E.Y., D. Ma, and H. Nikaido, AcrD of Escherichia coli is an aminoglycoside efflux pump. J Bacteriol, 2000. 182(6): p. 1754-6.

262. Paulsen, I.T., M.H. Brown, and R.A. Skurray, Proton-dependent multidrug efflux systems. Microbiol Rev, 1996. 60(4): p. 575-608.

263. Alguel, Y., et al., Crystal structures of multidrug binding protein TtgR in complex with antibiotics and plant antimicrobials. J Mol Biol, 2007. 369(3): p. 829-40.

264. Schumacher, M.A., M.C. Miller, and R.G. Brennan, Structural mechanism of the simultaneous binding of two drugs to a multidrug-binding protein. Embo J, 2004. 23(15): p. 2923-30.

265. Lomovskaya, O. and K. Lewis, Emr, an Escherichia coli locus for multidrug resistance. Proc Natl Acad Sci U S A, 1992. 89(19): p. 8938-42.

266. DeMarco, C.E., et al., Efflux-related resistance to norfloxacin, dyes, and biocides in bloodstream isolates of Staphylococcus aureus. Antimicrob Agents Chemother, 2007. 51(9): p. 3235-9.

267. Davidson, A.L., et al., Structure, function, and evolution of bacterial ATP-binding cassette systems. Microbiol Mol Biol Rev, 2008. 72(2): p. 317-64, table of contents.

268. Ambudkar, S.V., et al., P-glycoprotein: from genomics to mechanism. Oncogene, 2003. 22(47): p. 7468-85.

269. Lubelski, J., W.N. Konings, and A.J. Driessen, Distribution and physiology of ABC-type transporters contributing to multidrug resistance in bacteria. Microbiol Mol Biol Rev, 2007. 71(3): p. 463-76.

270. van Veen, H.W., C.F. Higgins, and W.N. Konings, Multidrug transport by ATP binding cassette transporters: a proposed two-cylinder engine mechanism. Res Microbiol, 2001. 152(3-4): p. 365-74.

271. Chami, M., et al., Three-dimensional structure by cryo-electron microscopy of $Y v c C$, an homodimeric ATP-binding cassette transporter from Bacillus subtilis. J Mol Biol, 2002. 315(5): p. 1075-85.

272. Dawson, R.J. and K.P. Locher, Structure of a bacterial multidrug ABC transporter. Nature, 2006. 443(7108): p. 180-5.

273. Kobayashi, N., K. Nishino, and A. Yamaguchi, Novel macrolide-specific ABC-type efflux transporter in Escherichia coli. J Bacteriol, 2001. 183(19): p. 5639-44.

274. Schuldiner, S., et al., Small is mighty: EmrE, a multidrug transporter as an experimental paradigm. News Physiol Sci, 2001. 16: p. 130-4. 
275. Paulsen, I.T., et al., A family of gram-negative bacterial outer membrane factors that function in the export of proteins, carbohydrates, drugs and heavy metals from gramnegative bacteria. FEMS Microbiol Lett, 1997. 156(1): p. 1-8.

276. Dinh, T., I.T. Paulsen, and M.H. Saier, Jr., A family of extracytoplasmic proteins that allow transport of large molecules across the outer membranes of gram-negative bacteria. J Bacteriol, 1994. 176(13): p. 3825-31.

277. Nikaido, H., Multidrug efflux pumps of gram-negative bacteria. J Bacteriol, 1996. 178(20): p. 5853-9.

278. Vaara, M., Antibiotic-supersusceptible mutants of Escherichia coli and Salmonella typhimurium. Antimicrob Agents Chemother, 1993. 37(11): p. 2255-60.

279. Nikaido, H., et al., Multidrug efflux pump AcrAB of Salmonella typhimurium excretes only those beta-lactam antibiotics containing lipophilic side chains. J Bacteriol, 1998. 180(17): p. 4686-92.

280. Mao, W., et al., On the mechanism of substrate specificity by resistance nodulation division (RND)-type multidrug resistance pumps: the large periplasmic loops of MexD from Pseudomonas aeruginosa are involved in substrate recognition. Mol Microbiol, 2002. 46(3): p. 889-901.

281. Lee, A., et al., Interplay between efflux pumps may provide either additive or multiplicative effects on drug resistance. J Bacteriol, 2000. 182(11): p. 3142-50.

282. Koronakis, V., et al., Crystal structure of the bacterial membrane protein TolC central to multidrug efflux and protein export. Nature, 2000. 405(6789): p. 914-9.

283. Murakami, S., et al., Crystal structure of bacterial multidrug efflux transporter AcrB. Nature, 2002. 419(6907): p. 587-93.

284. Yu, E.W., et al., Structural basis of multiple drug-binding capacity of the AcrB multidrug efflux pump. Science, 2003. 300(5621): p. 976-80.

285. Su, C.C., et al., Conformation of the AcrB multidrug efflux pump in mutants of the putative proton relay pathway. J Bacteriol, 2006. 188(20): p. 7290-6.

286. Seeger, M.A., et al., Structural asymmetry of AcrB trimer suggests a peristaltic pump mechanism. Science, 2006. 313(5791): p. 1295-8.

287. Aires, J.R. and H. Nikaido, Aminoglycosides are captured from both periplasm and cytoplasm by the AcrD multidrug efflux transporter of Escherichia coli. J Bacteriol, 2005. 187(6): p. 1923-9. 
288. Ziha-Zarifi, I., et al., In vivo emergence of multidrug-resistant mutants of Pseudomonas aeruginosa overexpressing the active efflux system MexA-MexB-OprM. Antimicrob Agents Chemother, 1999. 43(2): p. 287-91.

289. El'Garch, F., et al., Cumulative effects of several nonenzymatic mechanisms on the resistance of Pseudomonas aeruginosa to aminoglycosides. Antimicrob Agents Chemother, 2007. 51(3): p. 1016-21.

290. Poole, K., Efflux-mediated antimicrobial resistance. J Antimicrob Chemother, 2005. 56(1): p. 20-51.

291. Mazzariol, A., et al., High-level fluoroquinolone-resistant clinical isolates of Escherichia coli overproduce multidrug efflux protein AcrA. Antimicrob Agents Chemother, 2000. 44(12): p. 3441-3.

292. Aiello, A.E., E.L. Larson, and S.B. Levy, Consumer antibacterial soaps: effective or just risky? Clin Infect Dis, 2007. 45 Suppl 2: p. S137-47.

293. Morita, Y., et al., NorM of vibrio parahaemolyticus is an $\mathrm{Na}(+)$-driven multidrug efflux pump. J Bacteriol, 2000. 182(23): p. 6694-7.

294. Miyamae, S., et al., A MATE family multidrug efflux transporter pumps out fluoroquinolones in Bacteroides thetaiotaomicron. Antimicrob Agents Chemother, 2001. 45(12): p. 3341-6.

295. Morita, Y., et al., NorM, a putative multidrug efflux protein, of Vibrio parahaemolyticus and its homolog in Escherichia coli. Antimicrob Agents Chemother, 1998. 42(7): p. 1778-82.

296. Neyfakh, A.A., Natural functions of bacterial multidrug transporters. Trends Microbiol, 1997. 5(8): p. 309-13.

297. Zgurskaya, H.I. and H. Nikaido, Bypassing the periplasm: reconstitution of the AcrAB multidrug efflux pump of Escherichia coli. Proc Natl Acad Sci U S A, 1999. 96(13): p. 7190-5.

298. Ramos, J.L., et al., Mechanisms of solvent tolerance in gram-negative bacteria. Annu Rev Microbiol, 2002. 56: p. 743-68.

299. Barnett, M.J., et al., Nucleotide sequence and predicted functions of the entire Sinorhizobium meliloti pSymA megaplasmid. Proc Natl Acad Sci U S A, 2001. 98(17): p. $9883-8$.

300. Cox, J.S., et al., Complex lipid determines tissue-specific replication of Mycobacterium tuberculosis in mice. Nature, 1999. 402(6757): p. 79-83. 
301. Domenech, P., et al., The role of MmpL8 in sulfatide biogenesis and virulence of Mycobacterium tuberculosis. J Biol Chem, 2004. 279(20): p. 21257-65.

302. Kruh, G.D. and M.G. Belinsky, The MRP family of drug efflux pumps. Oncogene, 2003. 22(47): p. 7537-52.

303. Suvannasankha, A., et al., Breast cancer resistance protein (BCRP/MXR/ABCG2) in acute myeloid leukemia: discordance between expression and function. Leukemia, 2004. 18(7): p. 1252-7.

304. Riordan, J.R. and V. Ling, Purification of P-glycoprotein from plasma membrane vesicles of Chinese hamster ovary cell mutants with reduced colchicine permeability. J Biol Chem, 1979. 254(24): p. 12701-5.

305. Shen, D.W., et al., Human multidrug-resistant cell lines: increased mdr1 expression can precede gene amplification. Science, 1986. 232(4750): p. 643-5.

306. Kartner, N., J.R. Riordan, and V. Ling, Cell surface P-glycoprotein associated with multidrug resistance in mammalian cell lines. Science, 1983. 221(4617): p. 1285-8.

307. Kartner, N., et al., Detection of P-glycoprotein in multidrug-resistant cell lines by monoclonal antibodies. Nature, 1985. 316(6031): p. 820-3.

308. Chan, H.S., et al., A sensitive method for immunocytochemical detection of $P$ glycoprotein in multidrug-resistant human ovarian carcinoma cell lines. Lab Invest, 1988. 59(6): p. 870-5.

309. Litman, T., et al., From MDR to MXR: new understanding of multidrug resistance systems, their properties and clinical significance. Cell Mol Life Sci, 2001. 58(7): p. 931-59.

310. Lage, $\mathrm{H}$., ABC-transporters: implications on drug resistance from microorganisms to human cancers. Int J Antimicrob Agents, 2003. 22(3): p. 188-99.

311. Gottesman, M.M. and V. Ling, The molecular basis of multidrug resistance in cancer: the early years of P-glycoprotein research. FEBS Lett, 2006. 580(4): p. 998-1009.

312. Surowiak, P., et al., ABCC2 (MRP2, cMOAT) can be localized in the nuclear membrane of ovarian carcinomas and correlates with resistance to cisplatin and clinical outcome. Clin Cancer Res, 2006. 12(23): p. 7149-58.

313. Dietel, M., et al., Membrane vesicle formation due to acquired mitoxantrone resistance in human gastric carcinoma cell line EPG85-257. Cancer Res, 1990. 50(18): p. 61006. 
314. Chen, C., J. Zhou, and C. Ji, Quercetin: a potential drug to reverse multidrug resistance. Life Sci, 2010. 87(11-12): p. 333-8.

315. Walker, J.E., et al., Distantly related sequences in the alpha- and beta-subunits of ATP synthase, myosin, kinases and other ATP-requiring enzymes and a common nucleotide binding fold. Embo J, 1982. 1(8): p. 945-51.

316. Hyde, S.C., et al., Structural model of ATP-binding proteins associated with cystic fibrosis, multidrug resistance and bacterial transport. Nature, 1990. 346(6282): p. 362-5.

317. Fojo, A.T., et al., Expression of a multidrug-resistance gene in human tumors and tissues. Proc Natl Acad Sci U S A, 1987. 84(1): p. 265-9.

318. Goldstein, L.J., et al., Expression of a multidrug resistance gene in human cancers. J Natl Cancer Inst, 1989. 81(2): p. 116-24.

319. Cole, S.P., et al., Overexpression of a transporter gene in a multidrug-resistant human lung cancer cell line. Science, 1992. 258(5088): p. 1650-4.

320. Haimeur, A., et al., The MRP-related and BCRP/ABCG2 multidrug resistance proteins: biology, substrate specificity and regulation. Curr Drug Metab, 2004. 5(1): p. 21-53.

321. Nooter, K., et al., The prognostic significance of expression of the multidrug resistanceassociated protein (MRP) in primary breast cancer. Br J Cancer, 1997. 76(4): p. 48693.

322. Arts, H.J., et al., Drug resistance-associated markers P-glycoprotein, multidrug resistance-associated protein 1, multidrug resistance-associated protein 2, and lung resistance protein as prognostic factors in ovarian carcinoma. Clin Cancer Res, 1999. 5(10): p. 2798-805.

323. Taniguchi, K., et al., A human canalicular multispecific organic anion transporter (cMOAT) gene is overexpressed in cisplatin-resistant human cancer cell lines with decreased drug accumulation. Cancer Res, 1996. 56(18): p. 4124-9.

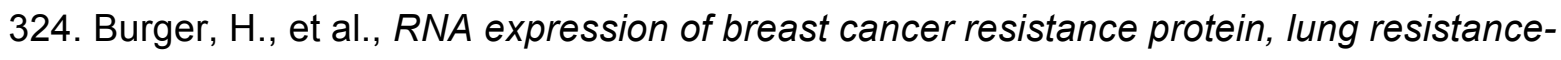
related protein, multidrug resistance-associated proteins 1 and 2, and multidrug resistance gene 1 in breast cancer: correlation with chemotherapeutic response. Clin Cancer Res, 2003. 9(2): p. 827-36.

325. Materna, V., et al., RNA expression of MDR1/P-glycoprotein, DNA-topoisomerase I, and MRP2 in ovarian carcinoma patients: correlation with chemotherapeutic response. Gynecol Oncol, 2004. 94(1): p. 152-60. 
326. Borst, P., C. de Wolf, and K. van de Wetering, Multidrug resistance-associated proteins 3, 4, and 5. Pflugers Arch, 2007. 453(5): p. 661-73.

327. Konig, J., et al., Characterization of the human multidrug resistance protein isoform MRP3 localized to the basolateral hepatocyte membrane. Hepatology, 1999. 29(4): p. 1156-63.

328. Faneyte, I.F., P.M. Kristel, and M.J. van de Vijver, Multidrug resistance associated genes MRP1, MRP2 and MRP3 in primary and anthracycline exposed breast cancer. Anticancer Res, 2004. 24(5A): p. 2931-9.

329. Ohishi, Y., et al., ATP-binding cassette superfamily transporter gene expression in human primary ovarian carcinoma. Clin Cancer Res, 2002. 8(12): p. 3767-75.

330. Konig, J., et al., Expression and localization of human multidrug resistance protein (ABCC) family members in pancreatic carcinoma. Int J Cancer, 2005. 115(3): p. 35967.

331. Calatozzolo, C., et al., Expression of drug resistance proteins Pgp, MRP1, MRP3, MRP5 and GST-pi in human glioma. J Neurooncol, 2005. 74(2): p. 113-21.

332. Kruh, G.D., et al., $A B C C 10, A B C C 11$, and $A B C C 12$. Pflugers Arch, 2007. 453(5): p. 675-84.

333. Oguri, T., et al., MRP8/ABCC11 directly confers resistance to 5-fluorouracil. Mol Cancer Ther, 2007. 6(1): p. 122-7.

334. Nies, A.T., et al., Immunolocalization of multidrug resistance protein 5 in the human genitourinary system. J Urol, 2002. 167(5): p. 2271-5.

335. Belinsky, M.G., et al., Characterization of the drug resistance and transport properties of multidrug resistance protein 6 (MRP6, ABCC6). Cancer Res, 2002. 62(21): p. 6172-7.

336. Matsuzaki, Y., et al., Tissue-specific expression of the ABCC6 gene. J Invest Dermatol, 2005. 125(5): p. 900-5.

337. Scheffer, G.L., et al., MRP6 (ABCC6) detection in normal human tissues and tumors. Lab Invest, 2002. 82(4): p. 515-8.

338. Hopper-Borge, E., et al., Analysis of the drug resistance profile of multidrug resistance protein 7 (ABCC10): resistance to docetaxel. Cancer Res, 2004. 64(14): p. 4927-30.

339. Doyle, L.A., et al., A multidrug resistance transporter from human MCF-7 breast cancer cells. Proc Natl Acad Sci U S A, 1998. 95(26): p. 15665-70. 
340. Allikmets, R., et al., A human placenta-specific ATP-binding cassette gene (ABCP) on chromosome 4q22 that is involved in multidrug resistance. Cancer Res, 1998. 58(23): p. 5337-9.

341. Miyake, K., et al., Molecular cloning of cDNAs which are highly overexpressed in mitoxantrone-resistant cells: demonstration of homology to $A B C$ transport genes. Cancer Res, 1999. 59(1): p. 8-13.

342. Lage, H. and M. Dietel, Effect of the breast-cancer resistance protein on atypical multidrug resistance. Lancet Oncol, 2000. 1: p. 169-75.

343. Maliepaard, M., et al., Subcellular localization and distribution of the breast cancer resistance protein transporter in normal human tissues. Cancer Res, 2001. 61(8): p. 3458-64.

344. Fetsch, P.A., et al., Localization of the ABCG2 mitoxantrone resistance-associated protein in normal tissues. Cancer Lett, 2006. 235(1): p. 84-92.

345. Jonker, J.W., et al., The breast cancer resistance protein BCRP (ABCG2) concentrates drugs and carcinogenic xenotoxins into milk. Nat Med, 2005. 11(2): p. 127-9.

346. van der Kolk, D.M., et al., Expression and activity of breast cancer resistance protein $(B C R P)$ in de novo and relapsed acute myeloid leukemia. Blood, 2002. 99(10): p. 3763-70.

347. Ross, D.D., et al., Expression of breast cancer resistance protein in blast cells from patients with acute leukemia. Blood, 2000. 96(1): p. 365-8.

348. Laing, N.M., et al., Amplification of the ATP-binding cassette 2 transporter gene is functionally linked with enhanced efflux of estramustine in ovarian carcinoma cells. Cancer Res, 1998. 58(7): p. 1332-7.

349. Steinbach, D., et al., ABCA3 as a possible cause of drug resistance in childhood acute myeloid leukemia. Clin Cancer Res, 2006. 12(14 Pt 1): p. 4357-63.

350. Izquierdo, M.A., et al., Overexpression of the ABC transporter TAP in multidrugresistant human cancer cell lines. Br J Cancer, 1996. 74(12): p. 1961-7.

351. Lage, $\mathrm{H}$., et al., Enhanced expression of human ABC-transporter tap is associated with cellular resistance to mitoxantrone. FEBS Lett, 2001. 503(2-3): p. 179-84.

352. Ruetz, S. and P. Gros, Phosphatidylcholine translocase: a physiological role for the mdr2 gene. Cell, 1994. 77(7): p. 1071-81.

353. Childs, S., et al., Taxol resistance mediated by transfection of the liver-specific sister gene of P-glycoprotein. Cancer Res, 1998. 58(18): p. 4160-7. 
354. Gerloff, T., et al., The sister of P-glycoprotein represents the canalicular bile salt export pump of mammalian liver. J Biol Chem, 1998. 273(16): p. 10046-50.

355. Yasui, K., et al., Alteration in copy numbers of genes as a mechanism for acquired drug resistance. Cancer Res, 2004. 64(4): p. 1403-10.

356. Paterson, J.K., et al., Human ABCB6 localizes to both the outer mitochondrial membrane and the plasma membrane. Biochemistry, 2007. 46(33): p. 9443-52.

357. Frank, N.Y., et al., ABCB5-mediated doxorubicin transport and chemoresistance in human malignant melanoma. Cancer Res, 2005. 65(10): p. 4320-33.

358. Schatton, T., et al., Identification of cells initiating human melanomas. Nature, 2008. 451(7176): p. 345-9.

359. Nieth, C. and H. Lage, Induction of the ABC-transporters Mdr1/P-gp (Abcb1), mrpl (Abcc1), and bcrp (Abcg2) during establishment of multidrug resistance following exposure to mitoxantrone. J Chemother, 2005. 17(2): p. 215-23.

360. Gillet, J.P., et al., Microarray-based detection of multidrug resistance in human tumor cells by expression profiling of ATP-binding cassette transporter genes. Cancer Res, 2004. 64(24): p. 8987-93.

361. Szakacs, G., et al., Predicting drug sensitivity and resistance: profiling ABC transporter genes in cancer cells. Cancer Cell, 2004. 6(2): p. 129-37.

362. Efferth, T., et al., Expression profiling of ATP-binding cassette transporters in childhood T-cell acute lymphoblastic leukemia. Mol Cancer Ther, 2006. 5(8): p. 1986-94.

363. Awasthi, S., et al., Transport of glutathione conjugates and chemotherapeutic drugs by RLIP76 (RALBP1): a novel link between G-protein and tyrosine kinase signaling and drug resistance. Int J Cancer, 2003. 106(5): p. 635-46.

364. Awasthi, Y.C., et al., The non-ABC drug transporter RLIP76 (RALBP-1) plays a major role in the mechanisms of drug resistance. Curr Drug Metab, 2007. 8(4): p. 315-23.

365. Hudson, M.E., et al., Identification of differentially expressed proteins in ovarian cancer using high-density protein microarrays. Proc Natl Acad Sci U S A, 2007. 104(44): p. 17494-9.

366. Hosoi, E., et al., Effect of MDR antagonists on the cidal activity of vincristine for cells expressing MDR-1 is superior to those expressing MRP. Int J Oncol, 1998. 13(2): p. 343-8.

367. Ambudkar, S.V., et al., Biochemical, cellular, and pharmacological aspects of the multidrug transporter. Annu Rev Pharmacol Toxicol, 1999. 39: p. 361-98. 
368. Krishna, R. and L.D. Mayer, Multidrug resistance (MDR) in cancer. Mechanisms, reversal using modulators of MDR and the role of MDR modulators in influencing the pharmacokinetics of anticancer drugs. Eur J Pharm Sci, 2000. 11(4): p. 265-83.

369. Germann, U.A., et al., Cellular and biochemical characterization of VX-710 as a chemosensitizer: reversal of P-glycoprotein-mediated multidrug resistance in vitro. Anticancer Drugs, 1997. 8(2): p. 125-40.

370. te Boekhorst, P.A., et al., Reversal of typical multidrug resistance by cyclosporin and its non-immunosuppressive analogue SDZ PSC 833 in Chinese hamster ovary cells expressing the mdr1 phenotype. Cancer Chemother Pharmacol, 1992. 30(3): p. 23842.

371. Wandel, C., et al., P-glycoprotein and cytochrome P-450 3A inhibition: dissociation of inhibitory potencies. Cancer Res, 1999. 59(16): p. 3944-8.

372. Bates, S., et al., A Phase I study of infusional vinblastine in combination with the Pglycoprotein antagonist PSC 833 (valspodar). Cancer, 2001. 92(6): p. 1577-90.

373. Martin, C., et al., The molecular interaction of the high affinity reversal agent XR9576 with P-glycoprotein. Br J Pharmacol, 1999. 128(2): p. 403-11.

374. Starling, J.J., et al., Pharmacological characterization of LY335979: a potent cyclopropyldibenzosuberane modulator of P-glycoprotein. Adv Enzyme Regul, 1997. 37: p. 335-47.

375. van Zuylen, L., et al., Development of multidrug-resistance convertors: sense or nonsense? Invest New Drugs, 2000. 18(3): p. 205-20.

376. Stewart, A., et al., Phase I trial of XR9576 in healthy volunteers demonstrates modulation of P-glycoprotein in CD56+ lymphocytes after oral and intravenous administration. Clin Cancer Res, 2000. 6(11): p. 4186-91.

377. Erlund, I., et al., Plasma concentrations of the flavonoids hesperetin, naringenin and quercetin in human subjects following their habitual diets, and diets high or low in fruit and vegetables. Eur J Clin Nutr, 2002. 56(9): p. 891-8.

378. Nguyen, H., S. Zhang, and M.E. Morris, Effect of flavonoids on MRP1-mediated transport in Panc-1 cells. J Pharm Sci, 2003. 92(2): p. 250-7.

379. Taur, J.S. and R. Rodriguez-Proteau, Effects of dietary flavonoids on the transport of cimetidine via P-glycoprotein and cationic transporters in Caco-2 and LLC-PK1 cell models. Xenobiotica, 2008. 38(12): p. 1536-50. 
380. Cooray, H.C., et al., Interaction of the breast cancer resistance protein with plant polyphenols. Biochem Biophys Res Commun, 2004. 317(1): p. 269-75.

381. Yoshimura, S., et al., Inhibitory effect of flavonoids on the efflux of $\mathrm{N}$-acetyl 5aminosalicylic acid intracellularly formed in Caco-2 cells. J Biomed Biotechnol, 2009. 2009: p. 467489.

382. Shapiro, A.B. and V. Ling, Extraction of Hoechst 33342 from the cytoplasmic leaflet of the plasma membrane by P-glycoprotein. Eur J Biochem, 1997. 250(1): p. 122-9.

383. Conseil, G., et al., Flavonoids: a class of modulators with bifunctional interactions at vicinal ATP- and steroid-binding sites on mouse P-glycoprotein. Proc Natl Acad Sci U S A, 1998. 95(17): p. 9831-6.

384. Stavri, M., L.J. Piddock, and S. Gibbons, Bacterial efflux pump inhibitors from natural sources. J Antimicrob Chemother, 2007. 59(6): p. 1247-60.

385. Stermitz, F.R., et al., Synergy in a medicinal plant: antimicrobial action of berberine potentiated by 5'-methoxyhydnocarpin, a multidrug pump inhibitor. Proc Natl Acad Sci U S A, 2000. 97(4): p. 1433-7.

386. Ball, A.R., et al., Conjugating berberine to a multidrug efflux pump inhibitor creates an effective antimicrobial. ACS Chem Biol, 2006. 1(9): p. 594-600.

387. Samosorn, S., et al., Antibacterial activity of berberine-NorA pump inhibitor hybrids with a methylene ether linking group. Bioorg Med Chem, 2009. 17(11): p. 3866-72.

388. Lomovskaya, O., et al., Identification and characterization of inhibitors of multidrug resistance efflux pumps in Pseudomonas aeruginosa: novel agents for combination therapy. Antimicrob Agents Chemother, 2001. 45(1): p. 105-16.

389. Nakayama, K., et al., MexAB-OprM-specific efflux pump inhibitors in Pseudomonas aeruginosa. Part 1: discovery and early strategies for lead optimization. Bioorg Med Chem Lett, 2003. 13(23): p. 4201-4.

390. Thorarensen, A., et al., 3-Arylpiperidines as potentiators of existing antibacterial agents. Bioorg Med Chem Lett, 2001. 11(14): p. 1903-6.

391. Bohnert, J.A. and W.V. Kern, Selected arylpiperazines are capable of reversing multidrug resistance in Escherichia coli overexpressing RND efflux pumps. Antimicrob Agents Chemother, 2005. 49(2): p. 849-52.

392. Nelson, M.L. and S.B. Levy, Reversal of tetracycline resistance mediated by different bacterial tetracycline resistance determinants by an inhibitor of the Tet(B) antiport protein. Antimicrob Agents Chemother, 1999. 43(7): p. 1719-24. 
393. Meier, A., et al., Genetic alterations in streptomycin-resistant Mycobacterium tuberculosis: mapping of mutations conferring resistance. Antimicrob Agents Chemother, 1994. 38(2): p. 228-33.

394. Gill, A.E. and S.G. Amyes, The contribution of a novel ribosomal S12 mutation to aminoglycoside resistance of Escherichia coli mutants. J Chemother, 2004. 16(4): p. $347-9$.

395. Unge, J., et al., The crystal structure of ribosomal protein L22 from Thermus thermophilus: insights into the mechanism of erythromycin resistance. Structure, 1998. 6(12): p. 1577-86.

396. Thompson, J., P.A. Skeggs, and E. Cundliffe, Methylation of 16 S ribosomal RNA and resistance to the aminoglycoside antibiotics gentamicin and kanamycin determined by DNA from the gentamicin-producer, Micromonospora purpurea. Mol Gen Genet, 1985. 201(2): p. 168-73.

397. Beauclerk, A.A. and E. Cundliffe, Sites of action of two ribosomal RNA methylases responsible for resistance to aminoglycosides. J Mol Biol, 1987. 193(4): p. 661-71.

398. Doi, Y. and Y. Arakawa, 16 S ribosomal RNA methylation: emerging resistance mechanism against aminoglycosides. Clin Infect Dis, 2007. 45(1): p. 88-94.

399. Jana, S. and J.K. Deb, Molecular targets for design of novel inhibitors to circumvent aminoglycoside resistance. Curr Drug Targets, 2005. 6(3): p. 353-61.

400. Mingeot-Leclercq, M.P., Y. Glupczynski, and P.M. Tulkens, Aminoglycosides: activity and resistance. Antimicrob Agents Chemother, 1999. 43(4): p. 727-37.

401. Karlowsky, J.A., et al., Altered denA and anr gene expression in aminoglycoside adaptive resistance in Pseudomonas aeruginosa. J Antimicrob Chemother, 1997. 40(3): p. 371-6.

402. Daigle, D.M., D.W. Hughes, and G.D. Wright, Prodigious substrate specificity of $A A C\left(6^{\prime}\right)-A P H\left(2^{\prime \prime}\right)$, an aminoglycoside antibiotic resistance determinant in enterococci and staphylococci. Chem Biol, 1999. 6(2): p. 99-110.

403. Azucena, E. and S. Mobashery, Aminoglycoside-modifying enzymes: mechanisms of catalytic processes and inhibition. Drug Resist Updat, 2001. 4(2): p. 106-17.

404. Kim, C., et al., Characterization of the bifunctional aminoglycoside-modifying enzyme ANT(3")-li/AAC(6')-IId from Serratia marcescens. Biochemistry, 2006. 45(27): p. 8368-77. 
405. Pedersen, L.C., M.M. Benning, and H.M. Holden, Structural investigation of the antibiotic and ATP-binding sites in kanamycin nucleotidyltransferase. Biochemistry, 1995. 34(41): p. 13305-11.

406. Hon, W.C., et al., Structure of an enzyme required for aminoglycoside antibiotic resistance reveals homology to eukaryotic protein kinases. Cell, 1997. 89: p. 887895.

407. Sakon, J., et al., Molecular structure of kanamycin nucleotidyltransferase determined to 3.0-A resolution. Biochemistry, 1993. 32(45): p. 11977-84.

408. Wright, G.D., A.M. Berghuis, and S. Mobashery, Aminoglycoside antibiotics. Structures, functions, and resistance. Adv Exp Med Biol, 1998. 456: p. 27-69.

409. McKay, G.A., et al., Recognition of aminoglycoside antibiotics by enterococcalstaphylococcal aminoglycoside 3'-phosphotransferase type Illa: role of substrate amino groups. Antimicrob Agents Chemother, 1996. 40(11): p. 2648-50.

410. Miller, G.H., et al., The most frequent aminoglycoside resistance mechanisms-changes with time and geographic area: a reflection of aminoglycoside usage patterns? Aminoglycoside Resistance Study Groups. Clin Infect Dis, 1997. 24 Suppl 1: p. S46-62.

411. Gates, C.A. and D.B. Northrop, Substrate specificities and structure-activity relationships for the nucleotidylation of antibiotics catalyzed by aminoglycoside nucleotidyltransferase 2"-I. Biochemistry, 1988. 27(10): p. 3820-5.

412. Gates, C.A. and D.B. Northrop, Determination of the rate-limiting segment of aminoglycoside nucleotidyltransferase 2 "-I by $\mathrm{pH}$ and viscosity-dependent kinetics. Biochemistry, 1988. 27(10): p. 3834-42.

413. Perdersen, L.C., M.M. Benning, and H.M. Holden, Structural investigation of the antibiotic and ATP-binding sites in kanamycin nucleotidyltransferase. Biochemistry, 1995. 34: p. 13305-13311.

414. Van Pelt, J.E., R. lyengar, and P.A. Frey, Gentamicin nucleotidyltransferase. Stereochemical inversion at phosphorus in enzymatic 2'-deoxyadenylyl transfer to tobramycin. J Biol Chem, 1986. 261(34): p. 15995-9.

415. Holm, L. and C. Sander, DNA polymerase beta belongs to an ancient nucleotidyltransferase superfamily. Trends Biochem Sci, 1995. 20(9): p. 345-7. 
416. Aravind, L. and E.V. Koonin, DNA polymerase beta-like nucleotidyltransferase superfamily: identification of three new families, classification and evolutionary history. Nucleic Acids Res, 1999. 27(7): p. 1609-18.

417. Kotra, L.P., J. Haddad, and S. Mobashery, Aminoglycosides: perspectives on mechanisms of action and resistance and strategies to counter resistance. Antimicrob Agents Chemother, 2000. 44(12): p. 3249-56.

418. Ferretti, J.J., K.S. Gilmore, and P. Courvalin, Nucleotide sequence analysis of the gene specifying the bifunctional 6'-aminoglycoside acetyltransferase 2"-aminoglycoside phosphotransferase enzyme in Streptococcus faecalis and identification and cloning of gene regions specifying the two activities. J Bacteriol, 1986. 167(2): p. 631-8.

419. Costa, Y., et al., Characterization of the chromosomal aac(6')-li gene specific for Enterococcus faecium. Antimicrob Agents Chemother, 1993. 37(9): p. 1896-903.

420. DiGiammarino, E.L., et al., Solution studies of isepamicin and conformational comparisons between isepamicin and butirosin $A$ when bound to an aminoglycoside 6'-N-acetyltransferase determined by NMR spectroscopy. Biochemistry, 1998. 37(11): p. 3638-44.

421. Bhatnagar, R.S., et al., Structure of N-myristoyltransferase with bound myristoylCoA and peptide substrate analogs. Nat Struct Biol, 1998. 5(12): p. 1091-7.

422. Weston, S.A., et al., Crystal structure of the anti-fungal target $N$-myristoyl transferase. Nat Struct Biol, 1998. 5(3): p. 213-21.

423. Dutnall, R.N., et al., Structure of the histone acetyltransferase Hat1: a paradigm for the GCN5-related N-acetyltransferase superfamily. Cell, 1998. 94(4): p. 427-38.

424. Taylor, S.S., et al., Structural framework for the protein kinase family. Annu Rev Cell Biol, 1992. 8: p. 429-62.

425. Rao, V.D., et al., Structure of type Ilbeta phosphatidylinositol phosphate kinase: a protein kinase fold flattened for interfacial phosphorylation. Cell, 1998. 94(6): p. 82939.

426. Daigle, D.M., G.A. McKay, and G.D. Wright, Inhibition of aminoglycoside antibiotic resistance enzymes by protein kinase inhibitors. J Biol Chem, 1997. 272(40): p. 24755-8.

427. Edson, R.S. and C.L. Terrell, The aminoglycosides: streptomycin, kanamycin, gentamicin, tobramycin, amikacin, netilmicin, and sisomicin. Mayo Clin Proc, 1987. 62(10): p. 916-20. 
428. King, J.W., et al., Alterations in the microbial flora and in the incidence of bacteremia at a university hospital after adoption of amikacin as the sole formulary aminoglycoside. Clin Infect Dis, 1992. 14(4): p. 908-15.

429. Mitchison, D.A., The diagnosis and therapy of tuberculosis during the past 100 years. Am J Respir Crit Care Med, 2005. 171(7): p. 699-706.

430. Wongsrichanalai, C., et al., Extensive drug resistance in malaria and tuberculosis. Emerg Infect Dis, 2010. 16(7): p. 1063-7.

431. Wilton, P., et al., Strategies to contain the emergence of antimicrobial resistance: a systematic review of effectiveness and cost-effectiveness. J Health Serv Res Policy, 2002. 7(2): p. 111-7.

432. Quigley, E.M., Probiotics in functional gastrointestinal disorders: what are the facts? Curr Opin Pharmacol, 2008. 8(6): p. 704-8.

433. Sayamov, R.M., Treatment and prophylaxis of cholera with bacteriophage. Bull World Health Organ, 1963. 28(3): p. 361-7.

434. Hyman, P. and S.T. Abedon, Bacteriophage host range and bacterial resistance. Adv Appl Microbiol, 2010. 70: p. 217-48.

435. Sette, A. and R. Rappuoli, Reverse vaccinology: developing vaccines in the era of genomics. Immunity, 2010. 33(4): p. 530-41.

436. Igaz, P., [Antimicrobial peptides: a new way to treat bacterial infections]. Orv Hetil, 2001. 142(4): p. 169-71.

437. Ghadiri, M.R., et al., Self-assembling organic nanotubes based on a cyclic peptide architecture. Nature, 1993. 366(6453): p. 324-7.

438. Fernandez-Lopez, S., et al., Antibacterial agents based on the cyclic D,L-alpha-peptide architecture. Nature, 2001. 412(6845): p. 452-5.

439. Fourmy, D., M.I. Recht, and J.D. Puglisi, Binding of neomycin-class aminoglycoside antibiotics to the A-site of 16 S rRNA. J Mol Biol, 1998. 277(2): p. 347-62.

440. Haddad, J., et al., Design of novel antibiotics that bind to the ribosomal acyltransfer site. J Am Chem Soc, 2002. 124(13): p. 3229-37.

441. Venot, A., et al., Disaccharide mimetics of the aminoglycoside antibiotic neamine. Chembiochem, 2004. 5(9): p. 1228-36.

442. Moazed, D. and H.F. Noller, Interaction of antibiotics with functional sites in $16 \mathrm{~S}$ ribosomal RNA. Nature, 1987. 327: p. 389-394. 
443. Wong, C.H., et al., Specificity of aminoglycoside antibiotics for the A-site of the decoding region of ribosomal RNA. Chem Biol, 1998. 5(7): p. 397-406.

444. Fourmy, D., S. Yoshizawa, and J.D. Puglisi, Paromomycin binding induces a local conformational change in the A-site of 16S rRNA. J Mol Biol, 1998. 277: p. 333-345.

445. Karimi, R. and M. Ehrenberg, Dissociation rate of cognate peptidyl-tRNA from the Asite of hyper-accurate and error-prone ribosomes. Eur J Biochem, 1994. 226: p. 355360.

446. Cabanas, M.J., D. Vazquez, and J. Modolell, Inhibition of ribosomal translocation by aminoglycoside antibiotics. Biochem. Biophys. Res. Commun., 1978. 83: p. 991-997.

447. Davies, J., L. Gorini, and B.D. Davis, Misreading of RNA codewords induced by aminoglycoside antibiotics. Mol Pharmacol, 1965. 1: p. 93-106.

448. Green, R. and H.F. Noller, Ribosomes and translation. Annu Rev Biochem, 1997. 66: p. 679-716.

449. Mehta, R. and W.S. Champney, Neomycin and paromomycin inhibit 30 S ribosomal subunit assembly in Staphylococcus aureus. Curr Microbiol, 2003. 47(3): p. 237-43.

450. Hermann, T., Strategies for the Design of Drugs Targeting RNA and RNA-Protein Complexes. Angew Chem Int Ed Engl, 2000. 39(11): p. 1890-1904.

451. Tok, J.B.H., J. Cho, and R.R. Rando, Aminoglycoside hybrids as potent RNA antagonists. Tetrahedron, 1999. 55(18): p. 5741-5758.

452. Michael, K., H. Wang, and Y. Tor, Enhanced RNA binding of dimerized aminoglycosides. Bioorg Med Chem, 1999. 7(7): p. 1361-71.

453. Tok, J.B. and G.R. Huffman, Enhanced binding of aminoglycoside dimers to a "dimerized" A-site 16S rRNA construct. Bioorg Med Chem Lett, 2000. 10(14): p. 1593-5.

454. Litovchick, A., A.G. Evdokimov, and A. Lapidot, Aminoglycoside-arginine conjugates that bind TAR RNA: synthesis, characterization, and antiviral activity. Biochemistry, 2000. 39(11): p. 2838-52.

455. Lee, J., et al., An approach to enhance specificity against RNA targets using heteroconjugates of aminoglycosides and chloramphenicol (or linezolid). J Am Chem Soc, 2004. 126(7): p. 1956-7.

456. Riguet, E., et al., Neamine dimers targeting the HIV-1 TAR RNA. Bioorg Med Chem Lett, 2005. 15(21): p. 4651-5. 
457. Ellington, A.D., RNA ligands: out of shape but fir for recognition. Curr Biol, 1993. 3(6): p. 375-7.

458. Wilson, D.S. and J.W. Szostak, In vitro selection of functional nucleic acids. Annu Rev Biochem, 1999. 68: p. 611-47.

459. Chow, C.S. and F.M. Bogdan, A Structural Basis for RNAminus signLigand Interactions. Chem Rev, 1997. 97(5): p. 1489-1514.

460. Walter, F., Q. Vicens, and E. Westhof, Aminoglycoside-RNA interactions. Curr Opin Chem Biol, 1999. 3(6): p. 694-704.

461. Zapp, M.L., S. Stern, and M.R. Green, Small molecules that selectively block RNA binding of HIV-1 Rev protein inhibit Rev function and viral production. Cell, 1993. 74(6): p. 969-78.

462. Wang, S., et al., Binding of neomycin to the TAR element of HIV-1 RNA induces dissociation of Tat protein by an allosteric mechanism. Biochemistry, 1998. 37(16): p. 5549-57.

463. Hermann, T. and E. Westhof, RNA-binding Antibiotics. 1999, European Molecular Biology Organization.

464. Mei, H.Y., et al., Inhibition of an HIV-1 Tat-derived peptide binding to TAR RNA by aminoglycoside antibiotics. Bioorg Med Chem Lett, 1995. 5: p. 2755-2760.

465. Faber, C., et al., Structural rearrangements of HIV-1 Tat-responsive RNA upon binding of neomycin B. J Biol Chem, 2000. 275(27): p. 20660-6.

466. Tok, J.B., J. Cho, and R.R. Rando, Aminoglycoside antibiotics are able to specifically bind the 5'-untranslated region of thymidylate synthase messenger RNA.

Biochemistry, 1999. 38(1): p. 199-206.

467. von Ahsen, U., J. Davies, and R. Schroeder, Antibiotic inhibition of group I ribozyme function. Nature, 1991. 353: p. 368-370.

468. von Ahsen, U., J. Davies, and R. Schroeder, Non-competitive inhibition of group I intron RNA self-splicing by aminoglycoside antibiotics. J Mol Biol, 1992. 226: p. 935-941.

469. Mikkelsen, N.E., et al., Inhibition of RNase P RNA cleavage by aminoglycosides. Proc Natl Acad Sci USA, 1999. 96: p. 6155-6160.

470. Mikkelsen, N.E., et al., Aminoglycoside binding displaces a divalent metal ion in a tRNA-neomycin B complex. Nat Struct Biol, 2001. 8(6): p. 510-4. 
471. Huang, L., A. Serganov, and D.J. Patel, Structural insights into ligand recognition by a sensing domain of the cooperative glycine riboswitch. Mol Cell, 2010. 40(5): p. 77486.

472. Garst, A.D., et al., Crystal structure of the lysine riboswitch regulatory mRNA element. J Biol Chem, 2008. 283(33): p. 22347-51.

473. Edwards, A.L., et al., Structural basis for recognition of S-adenosylhomocysteine by riboswitches. RNA, 2010. 16(11): p. 2144-55.

474. Batey, R.T., S.D. Gilbert, and R.K. Montange, Structure of a natural guanineresponsive riboswitch complexed with the metabolite hypoxanthine. Nature, 2004. 432(7015): p. 411-5.

475. Serganov, A., et al., Structural basis for discriminative regulation of gene expression by adenine- and guanine-sensing mRNAs. Chem Biol, 2004. 11(12): p. 1729-41.

476. Jenkins, J.L., et al., Comparison of a preQ1 riboswitch aptamer in metabolite-bound and free states with implications for gene regulation. J Biol Chem, 2011. 286(28): p. 24626-37.

477. Klein, D.J., T.E. Edwards, and A.R. Ferre-D'Amare, Cocrystal structure of a class I preQ1 riboswitch reveals a pseudoknot recognizing an essential hypermodified nucleobase. Nat Struct Mol Biol, 2009. 16(3): p. 343-4.

478. Smith, K.D., et al., Structural and biochemical determinants of ligand binding by the cdi-GMP riboswitch. Biochemistry, 2010. 49(34): p. 7351-9.

479. Montange, R.K. and R.T. Batey, Structure of the S-adenosylmethionine riboswitch regulatory mRNA element. Nature, 2006. 441(7097): p. 1172-5.

480. Stoddard, C.D., et al., Free state conformational sampling of the SAM-I riboswitch aptamer domain. Structure, 2010. 18(7): p. 787-97.

481. Lu, C., et al., SAM recognition and conformational switching mechanism in the Bacillus subtilis yitJ S box/SAM-I riboswitch. J Mol Biol, 2010. 404(5): p. 803-18.

482. Gilbert, S.D., et al., Structure of the SAM-II riboswitch bound to S-adenosylmethionine. Nat Struct Mol Biol, 2008. 15(2): p. 177-82.

483. Lu, C., et al., Crystal structures of the SAM-III/S(MK) riboswitch reveal the SAMdependent translation inhibition mechanism. Nat Struct Mol Biol, 2008. 15(10): p. 1076-83.

484. Serganov, A., L. Huang, and D.J. Patel, Coenzyme recognition and gene regulation by a flavin mononucleotide riboswitch. Nature, 2009. 458(7235): p. 233-7. 
485. Thore, S., M. Leibundgut, and N. Ban, Structure of the eukaryotic thiamine pyrophosphate riboswitch with its regulatory ligand. Science, 2006. 312(5777): p. 1208-11.

486. Serganov, A., et al., Structural basis for gene regulation by a thiamine pyrophosphatesensing riboswitch. Nature, 2006. 441(7097): p. 1167-71.

487. Klein, D.J., et al., Requirement of helix P2.2 and nucleotide G1 for positioning the cleavage site and cofactor of the glmS ribozyme. J Mol Biol, 2007. 373(1): p. 178-89.

488. Dann, C.E., 3rd, et al., Structure and mechanism of a metal-sensing regulatory RNA. Cell, 2007. 130(5): p. 878-92.

489. Duchardt-Ferner, E., et al., Highly modular structure and ligand binding by conformational capture in a minimalistic riboswitch. Angew Chem Int Ed Engl, 2010. 49(35): p. 6216-9. 


\title{
CHAPTER 3
}

\section{FACTORS THAT INFLUENCE SELECTIVITY OF THE NEOMYCIN-B RNA APTAMER}

\author{
A paper to be submitted to Nucleic Acid Research \\ Muslum Ilgu, Ragothaman M Yennamalli ${ }^{\$}$ Megan M. Kleckler, Monica H. Lamm, \\ Taner Z. Sen and Marit Nilsen-Hamilton*
}

${ }^{\$}$ Current address: 440 Henry Mall, University of Wisconsin-Madison, Madison WI 53706

${ }^{*}$ Corresponding author 


\begin{abstract}
Nucleic acid aptamers are frequently characterized as highly specific for their ligands, which implies precise molecular recognition between interacting groups on the aptamer and ligand. However, as short oligonucleotides, aptamers are likely to be structurally flexible, which suggests they might readily adjust to alternative ligands and therefore have low specificity. To understand the role of aptamer structure in establishing ligand specificity, we examined the neomycin-B aptamer, which has been reported as highly selective for neomycin-B over other aminoglycosides. Experimental values for ligand specificity correlated well, for almost all aminoglycosides, with the relative affinity scores from docking analysis with the aptamer considered rigid. However, the aptamer specificity was much less constrained than previously reported. Consistent with this finding, the molecular dynamics simulations predicted a very flexible binding pocket and pentaloop of the unoccupied aptamer and that the A16 in the pentaloop of the aptamer interact with the ligand as shown previously by NMR. To understand how the flexible pocket and A16 could collaborate to define ligand specificity, we examined the effect of ionic environment with aptamer variants containing replacement bases or a deletion at position 16, with 2-aminopurine substituted aptamer analogs and with NMR spectrometry. The configuration of A16 in the aptamer was found to be ligand dependent and strongly affected by the ionic environment. Contrary to what was predicted by previous NMR spectroscopy results, the presence of A16 in the pentaloop had no impact on the aptamer's affinity for neomycin-B. However, the $A$ in this position appears to be responsible for the broader ligand specificity and large differences in affinity and ligand specificities between buffer conditions. The results of this study demonstrate that to understand the structural contributions of an aptamer to its affinity and specificity for ligands requires investigation of the structural dynamics in a variety of ionic environments.
\end{abstract}




\section{INTRODUCTION}

Nucleic acids, especially RNAs, have diverse functions in living organisms that frequently involve conformational rearrangements during ligand binding and signaling, cotranscriptional folding, catalysis in ribozymes and the assembly of ribonucleoproteins (AlHashimi and Walter 2008; Nick Taylor et al. 2008; Forster et al. 2012). Alternatively, interaction between RNAs and inhibitory molecules can shift the RNA conformation to become nonfunctional as for the interaction between ribosomal RNA and aminoglycosides.

Aminoglycosides are an important class of broad-spectrum antibiotics that target the A-site of the ribosome where mRNA and tRNA come to close proximity for the amino acid transfer of prokaryotic ribosomes and interfere with protein synthesis (Dahlberg 1989). As a result of this binding, the helix-44 constituting the A-site is distorted and the genetic code is misread leading to ceasing of translation and death of bacteria (Moazed and Noller 1987). However, aminoglycosides also interact with a variety of other natural RNAs including Revresponsive element (RRE) (Zapp et al. 1993; Wang et al. 1997) and trans-activating region (TAR) of HIV-1 (Wang et al. 1998), the hepatitis delta virus (HDV) ribozyme (Rogers et al. 1996; Chia et al. 1997), hammerhead (HH) ribozyme (Stage et al. 1995; Tor et al. 1998), RNase P (Mikkelsen et al. 1999), iron response element (IRE) (Lee et al. 2004), the site 1 mRNA of thymidylate synthase (TS) (Tok et al. 1999), group 1 self-splicing introns (von Ahsen et al. 1991; von Ahsen et al. 1992) and tRNA ${ }^{\text {Phe }}$ (Mikkelsen et al. 2001). Some of these interactions cause their toxicity and so limit the usefulness of these otherwise very effective antibiotics.

To understand the interaction between RNA and aminoglycosides, RNA aptamers were selected for neomycin-B (Wallis et al. 1995), tobramycin (Wang et al. 1996; Werstuck

and Green 1998), kanamycin-A (Lato et al. 1995; Werstuck and Green 1998) and kanamycin-B (Kwon et al. 2001). Among these and the naturally occurring RNAs, a 23mer 
RNA aptamer selected against neomycin-B is the smallest RNA motif reported to have high affinity and specificity for neomycin-B over paromomycin (Wallis and Schroeder 1997; Cowan et al. 2000; de-los-Santos-Alvarez et al. 2007; Stampfl et al. 2007; de-los-SantosAlvarez et al. 2009). Other aminoglycoside-binding RNA aptamers have been reported with different specificities than for their original targets, such as the lividomycin aptamer that recognizes neomycin-B and paromomycin equally well (Lato et al. 1995), the W13 RNA that binds tobramycin and neomycin-B with equal affinity (Wang and Rando 1995) and the kanamycin-B aptamer that recognizes aminoglycosides in the order tobramycin>kanamycinB>neomycin-B (Kwon et al. 2001).

In this study, we have examined the structural basis of the specificity of the 23 mer neomycin-B aptamer by an integrated experimental and computational approach. Although an NMR-derived structure is available for the aptamer in complex with neomycin-B, there is no equivalent structure for the aptamer in the absence of ligand. Therefore, we simulated the unoccupied aptamer structure using molecular dynamics (MD) software. In these simulations adenosine (A16) in the pentaloop that is found in the NMR structure flipped over the binding pocket with hydrogen bonds to the fourth sugar ring of neomycin-B was found to be very mobile. Contrary to the NMR results, the MD simulation and molecular docking analysis predicted that $\mathrm{A} 16$ interacts with the nonpolar face of the sugar moiety rather than through hydrogen bonds. Experimental analysis of a 2-aminopurine substituted aptamer by fluorescence and isothermal titration calorimetry (ITC) were consistent with these predictions for the interaction with paromomycin and with the likelihood that A16 exists in a stacked condition in the pentaloop of the unoccupied aptamer. Experimental studies of aptamers with various bases in position 16 demonstrated a variable dependence on the nature of the base or its absence for affinity to different aminoglycosides. In these studies, the aptamer also demonstrated a different profile of specificity for neomycin-B versus paromomycin 
compared with that previously reported (Wallis et al. 1995). Follow-up studies with a series of 10 aminoglycosides indicated that the aptamer can recognize a variety of aminoglycosides with similar binding affinities. These measured affinities were wellcorrelated with the computationally determined molecular docking scores. Further studies to examine the question of why the current studies showed a broad aminoglycoside specificity compared with previous studies of others that demonstrated a narrow specificity for neomycin-B revealed the significant impact of buffer constituents. Thus, our results demonstrate that the interaction of this short RNA with aminoglycosides is influenced by its ionic environment, depends variably on different structural motifs. 


\section{MATERIALS AND METHODS}

\section{Chemicals and RNAs}

Neomycin-B, paromomycin, ribostamycin, tobramycin, kanamycin-A, kanamycin-B, sisomicin, geneticin, netilmicin and amikacin were obtained as their sulfate salts from Sigma-Aldrich (St. Louis, MO). All RNA oligos were purchased from Integrated DNA Technologies Inc. (Coralville, IA) and they were kept at $-20^{\circ} \mathrm{C}$ in $d_{d d H_{2}} \mathrm{O}$ until used in the isothermal titration calorimetry (ITC) or in 2AP fluorescence experiments. Sodium cacodylate was purchased from Sigma-Aldrich (St. Louis, MO) and cacodylic acid was from Amresco (Solon, $\mathrm{OH}$ ). All other chemicals used to prepare the buffers were obtained from Fisher Scientific (Pittsburgh, PA).

\section{Isothermal titration calorimetry (ITC)}

ITC experiments were performed on a Microcal VP-ITC microcalorimeter (Northampton, MA), which was calibrated using the built-in electrical calibration check. They were conducted in buffer $\mathrm{A}\left(13.5 \mathrm{mM} \mathrm{NaCl}, 150 \mathrm{mM} \mathrm{KCl}, 20 \mathrm{mM}\right.$ HEPES, $0.22 \mathrm{mM} \mathrm{Na}_{2} \mathrm{HPO}_{4}$, $0.44 \mathrm{mM} \mathrm{KH}_{2} \mathrm{PO}_{4}, 120 \mu \mathrm{M} \mathrm{MgCl}_{2}, 120 \mathrm{nM} \mathrm{CaCl} 2,100 \mu \mathrm{M} \mathrm{MgSO}_{4}$ in $\mathrm{ddH}_{2} \mathrm{O}$ at $\mathrm{pH} 7.3$ at $25^{\circ} \mathrm{C}$ ), buffer $\mathrm{B}\left(10 \mathrm{mM} \mathrm{Na}_{2} \mathrm{HPO}_{4}\right.$ at $\mathrm{pH}$ 6.1), buffer $\mathrm{C}\left(10 \mathrm{mM} \mathrm{Na} \mathrm{HPO}_{4}\right.$ at $\left.\mathrm{pH} 7.3\right)$, buffer $\mathrm{D}(10$ $\mathrm{mM} \mathrm{Na}_{2} \mathrm{HPO}_{4}, 5 \mathrm{mM} \mathrm{MgCl} 2$ at pH 7.3), or buffer $\mathrm{E}\left(5 \mathrm{mM} \mathrm{MgCl}_{2}, 200 \mathrm{mM} \mathrm{NH}_{4} \mathrm{Cl}, 80 \mathrm{mM} \mathrm{KCl}\right.$, $80 \mathrm{mM} \mathrm{Na-cacodylate}$ at $\mathrm{pH}$ 7.4). For an individual experiments, both the RNAs in the reaction cell and the aminoglycosides (neomycin-B, paromomycin, ribostamycin, tobramycin, kanamycin-A, kanamycin-B, sisomicin, geneticin, netilmicin and amikacin) in the syringe were prepared in the same buffer. All solutions were degassed at room temperature immediately prior to use in the experiment. Following thermal equilibrium at $25^{\circ} \mathrm{C}$, an initial 60 second delay and a single $1.0 \mu \mathrm{L}$ titrant injection, 30 serial injections of $10 \mu \mathrm{L}$ aminoglycoside was added at an interval of 150 seconds into the stirred sample cell $(1.4 \mathrm{~mL})$ 
containing the RNA oligos at a stirring rate of $310 \mathrm{rpm}$ at $25^{\circ} \mathrm{C}$. The heat associated with each titration peak was integrated and plotted against the respective molar ratio of aminoglycoside and RNA. To correct for heats of dilution from titrants, control experiments were performed by making identical injections of the titrant solution into a cell containing only the respective buffer and these values were subtracted from the titration of the titrant solution into the reaction cell. Data were analyzed using nonlinear least-squares curve fitting in Origin7.0 (OriginLab Corp., Northampton, MA) using the standard one-binding site or two-binding site models supplied with Origin 7.0

\section{Molecular docking of aminoglycosides to the neomycin RNA aptamer via DOCK}

\section{Preparation of the RNA}

The RNA aptamer structure was obtained from Protein Data Bank (REF: PMID: 10592235) (PDB, pdb id: 1nem (REF: PMID: 10425683)). After visualizing all the models in the pdb file the coordinates of the $5^{\text {th }}$ model of NMR was used in this study. The Dock Prep module of Chimera $\vee 1.5 .2$ (Pettersen et al. 2004) was used to prepare the aptamer for docking. The steps include removing water molecules, addition of hydrogen atoms, assigning partial charges (using AMBER parm99 partial charges) and saving the receptor in two formats; pdb and Tripos Mol2.

\section{Preparation of aminoglycoside antibiotics}

Ten aminoglycosides were selected for this study; amikacin, geneticin, kanamycin-A, kanamycin-B, neomycin-B, netilmicin, paromomycin, ribostamycin, sisomicin, and tobramycin. A relative docking score for each aminoglycoside was established against that of neomycin-B. Preparation of the ligands for docking involved the addition of hydrogens and assigning partial Gasteiger charges using BABEL v 1.6 (CCL.NET). 


\section{Active site identification and molecular docking}

The ligand (neomycin-B) in complex with the RNA as given in the pdb file was used to define the active site. Atoms within a radius of $10 \AA$ from neomycin-B were defined as the binding site (or active site) using the module SPHGEN of DOCK v6.2 (Kuntz et al. 1982).

Molecular docking was performed using DOCK v6.2 (Kuntz et al. 1982), in which the first step is to construct a three-dimensional grid around the active site with a spacing of $0.3 \AA$. The grid helps in the next steps of docking that includes but not limited to calculating the binding energies, avoiding steric clashes, etc. The incremental construction method of "anchor-and-grow" was used to identify the most probable conformation of the ligand. In this method, the largest substructure of the ligand (for example an aromatic ring) is first identified and placed in the active site as the so-called "anchors". The flexible elements (for example linking regions between aromatic rings) are then incrementally added, and each increment is evaluated for the lowest binding energy and minimal steric clash. At this stage the anchors are held rigid and various orientations of flexible parts are allowed to "grow". Through such an "anchor-and-grow" method the entire conformational search space of the aminoglycoside is explored. The parameters used in the molecular docking are given in Appendix S1. For each ligand, 1000 orientations were considered and the highly ranked conformation of the ligand along with the DOCK score is given as output.

\section{Post-docking analysis : Use of Ligplot to map the RNA-aminoglycoside interactions}

Ligplot v4.5.3 (Wallace et al. 1995) was used to map the interactions between the aptamer and the aminoglycosides and to validate the docking predictions. Specifically, Ligplot plots the hydrogen bonds and hydrophobic contacts between the receptor and ligand from a given $\mathrm{pdb}$. It also gives a flattened $2 \mathrm{D}$ plot of the various residues of the receptor interacting with the ligand. 


\section{Measurement of 2-aminopurine (2AP) fluorescence intensity}

To determine the effect of aminoglycoside binding on the pentaloop structure of the neomycin aptamer, 2AP was introduced to replace adenosine at position 16 . For binding assays $1.0 \mu \mathrm{M}$ of the neomycin aptamer was mixed with various concentrations of neomycin-B $(0-10 \mu \mathrm{M})$, paromomycin $(0-40 \mu \mathrm{M})$, or ribostamycin $(0-10 \mu \mathrm{M})$ in buffer $A$, $B, C, D$ and $E$. The binding of aminoglycosides was monitored by 2AP fluorescence using a Cary Eclipse spectrofluorometer (Varian, Palo Alto, CA). RNA alone in the buffer was subtracted from the raw data. Quartz cells with $1 \mathrm{~cm}$ path lengths were used for excitation and emission spectra that were acquired from $255 \mathrm{~nm}$ to $350 \mathrm{~nm}$ and $320 \mathrm{~nm}$ to $500 \mathrm{~nm}$, respectively to establish the maximum excitation and emission wavelengths for further studies. For all further measurements the excitation wavelength ( $\lambda$ ex) was $307 \mathrm{~nm}$ and the emission wavelength ( $\lambda$ em) was $370 \mathrm{~nm}$ and the emission slit was $5 \mathrm{~nm}$. The effects of aminoglycosides binding to the aptamer on 2AP fluorescence were fit to the following equation by a nonlinear regression function using CoStat (CoHort software, Monterey, CA): $\Delta \mathrm{F} / \Delta \mathrm{Fmax}=\mathrm{a}^{*}[$ aminoglycoside $] /(\mathrm{Kd}+[$ aminoglycoside $]) ;$ where, $\Delta \mathrm{F}$ is the fluorescence change of $\mathrm{F} 370$ (for 2AP), $\Delta \mathrm{Fmax}$ is the maximal 2AP fluorescence change of the bound

compared to the free state. [aminoglycoside] is the concentration of aminoglycoside, " $K_{d}$ " is the dissociation constant for the neomycin aptamer aminoglycoside interaction and "a" is a constant.

\section{Molecular dynamics simulations using GROMACS}

Coordinates of the neomycin-B RNA aptamer were taken from model 5 of the experimental NMR structure (Protein Data Bank (PDB) code: 1nem) (Jiang et al. 1999). 


\section{Preparing Neomycin-B for MD simulations}

Force field parameters for neomycin-B were obtained from SwissParam (Zoete et al. 2011), which provides topology and parameters for small organic molecules compatible with both the CHARMM22 all atom force field and GROMACS. The data was derived from the Merck Molecular ForceField (MMFF) and the dihedral angle terms were taken, as is, where the harmonic part of the bond, angle and improper terms were retained. Van der Waals parameters were taken from the closest atom type in CHARMM22 and the charges were obtained from MMFF. Generally, these estimated charges are not accurate enough to use in MD simulations. Therefore, the partial charges were calculated with GAMESS (General Atomic and Molecular Electronic Structure System) by fitting the electrostatic potentials obtained from the quantum mechanical ab initio calculations at the HF/6-31G* level (Spackman 1996). 


\section{RESULTS}

\section{Broad aminoglycoside specificity of the neomycin-B aptamer}

With the goal of utilizing the 23 mer neomycin-B aptamer in an intracellular environment, under which conditions it has been previously applied, we tested the affinity and specificity of this aptamer using buffer conditions that resemble those inside the cell (Buffer A). To our surprise, the relative dissociation constants $\left(K_{d} s\right)$ of this aptamer for neomycin-B and paromomycin were quite different from those previously reported (Wallis et al. 1995; Wallis and Schroeder 1997). Whereas it had previously been found that the aptamer is highly specific for neomycin-B, binding paromomycin with more than 100 -fold lower affinity, we observed only a 5 -fold difference in $\mathrm{K}_{d}$ between paromomycin and neomycin-B in buffer $A$. The $K_{d}$ for paromomycin was $1.4 \pm 0.19$ ( $N=5$ independent estimates) compared with $0.29 \pm 0.06(\mathrm{~N}=8)$ for neomycin-B. To further examine the aptamer specificity, eight more ligands were tested from both neomycin- and kanamycinfamily aminoglycosides (Fig. 1A) with the result that the aptamer can recognize both family members of the aminoglycosides (Table S1).

\section{Molecular docking and MD simulations predict a hydrophobic interaction between A16 and the sugar and a flexible pentaloop}

To develop a better understanding of the interaction of the neomycin-B aptamer (Fig. 1B) and aminoglycosides, we explored the use of molecular docking analysis in which the RNA was considered as a rigid body and the aminoglycosides as explicitly-protonated with rotational freedom. The results of this analysis showed a correlation between the experimental results and the docking scores for 9 of the 10 aminoglycosides tested with an $\mathrm{R}^{2}$ of 0.71 (Fig. 1C, Table S2). The exception was ribostamycin, for which the results significantly deviated from the derived linear fit (Fig. 1C, gray triangle). Unlike other 
neomycin-family aminoglycoside tested in this study, ribostamycin is missing the fourth ring (Fig. 1A). The very similar binding affinity between ribostamycin and neomycin-B determined by ITC suggests that ring IV plays a minor role in RNA binding, consistent with observations from the NMR study (Jiang et al. 1999).

When the NMR and molecular docking results were compared, a significant difference between them was how the adenosine at position 16 (A16) in the pentaloop interacts with the aminoglycoside. Whereas the NMR spectra showed hydrogen bonding between the base and neomycin-B, a Ligplot analysis of the aptamer-aminoglycoside predicted the A16 to make hydrophobic contacts with the aminoglycosides (Fig. S3). To investigate further this interaction between the aptamer and aminoglycosides, we used MD simulation with force fields for the neomycin-B inserted (Wallis and Schroeder 1997). As for the docking analysis, the A16 was also predicted by MD to interact with the nonpolar face of the sugar ring rather than by hydrogen bonding as shown by NMR.

The unoccupied aptamer was also simulated by MD after removing the neomycin-B coordinates. A comparison of the simulations of the occupied and unoccupied aptamers showed a flexible binding pocket and a large RMSD for A16 in the pentaloop reflecting a relatively wide dynamic range for this base in the unoccupied aptamer that is restricted when neomycin-B is in the binding pocket (Fig. 2).

\section{Experimental analysis of an aptamer with 2-aminopurine in position 16 identifies a shift in the environment of A16 with ligand binding}

To test the predictions made by the Ligplot and MD analysis that $A 16$ is very mobile and interacts with the aminoglycoside on the nonpolar face of the fourth ring, we created an aptamer with the A16 replaced by 2-aminopurine (2AP). A mobile base that interacts by hydrophobic forces with the aminoglycoside is expected to increase in fluorescence upon 
ligand binding. A large increase in 2AP fluorescence was observed with the binding of neomycin-B and other aminoglycosides. Using this fluorescent response, we determined the $K_{d} S$ for neomycin-B, paromomycin and ribostomycin (Fig. $3 A$ ). Although the $K_{d} S$ determined for neomycin-B and paromomycin matched well with the ITC results, the value obtained for ribostamycin did not (Fig. 3B, Table S1 and S5). However, the $\mathrm{K}_{d}$ determined by $2 A P$ fluorescence moved the calculated $\Delta G$ for ribostamycin more in line with the other aminoglycosides in relation to the docking scores (Fig. 3C).

\section{Effects of buffer components on the aptamer-aminoglycoside specificity}

Neither the DOCK analysis nor the MD simulations explained why the originally reported aminoglycoside specificity of this neomycin-B RNA aptamer was so different from that observed here. Consequently, we explored the possibility that the buffer constituents might affect specificity and used the 2AP16 aptamer to test the binding of aminoglycosides the buffers used in previous studies (Fig. 4). High specificity for neomycin-B over paromomycin and ribostamycin was confirmed in cacodylate buffer (E) as previously reported (Wallis et al. 1995). However, in the phosphate buffer (B) used for NMR structural determination (Jiang et al. 1999) the aptamer was less specific for neomycin-B over paromomycin as found in the buffer $(A)$ used for our study. The $\mathrm{pH}$ does not seem a factor in the specificity difference for aminoglycoside as the aptamer showed similar lack of specificity in the NMR buffer composition at pH 6.1 and 7.3 (Fig. 4 compare buffers $\mathrm{B}$ and $\mathrm{C}$ ).

The possibility of a pH effect on aptamer binding was also tested with ITC (Fig. 5A). Here it was found that the ITC titrations displayed a nonhyperbolic profile with a large heat release in the early portion of the titration up to a molar ratio of 1 , after which a normal titration curve was observed. Such nonhyperbolic titration profiles suggest the possibility of 
a significant structural change in the aptamer prior to binding to the aminoglycoside and therefore a considerable entropic component to the binding interaction.

Magnesium ions have been shown to promote folding by lowering the entropic cost (Fiore et al. 2012). Therefore, we tested the effect of $5 \mathrm{mM} \mathrm{MgCl} 2$ at $\mathrm{pH} 7.3$ (buffer D) on aptamer binding to neomycin-B, paromomycin and ribostamycin (Fig. 6B). For each aminoglycoside, the presence of $\mathrm{MgCl}_{2}$ shifted the titration curve toward a hyperbolic profile. These results suggest that the interaction of aptamer with aminoglycosides includes a large entropic cost that is minimized by the presence of $\mathrm{MgCl}_{2}$.

\section{The role of the A16 in establishing specificity of aminoglycoside binding}

The prominent position of A16 in the NMR structure, being flipped over neomycin-B, suggests that it might be important for establishing affinity and perhaps specificity of aminoglycoside binding. We tested this hypothesis by comparing the abilities of four aptamer variants in this position to bind aminoglycosides. Binding to neomycin-B and paromomycin was largely unaffected by changing the base in position 16. Even deleting the base did not decrease affinity of the aptamer for neomycin-B although it decreased the affinity for paromomycin by 5-fold (Fig. 6, Table S4). By contrast, changing the base decreased the affinity for ribostamycin by 5-fold (A16G) and 7-fold (A16U and A16C) and deleting the base dramatically decreased the affinity for ribostamycin by 40 -fold (Fig. 6 , Table S4). 


\section{DISCUSSION}

Our study of the 23mer neomycin-B RNA aptamer showed the expected high affinity binding with neomycin-B, but also identified an unexpectedly broad aminoglycoside specificity. Previous NMR studies of the structure of the aptamer occupied by neomycin-B demonstrated that binding to aminoglycosides by this aptamer is largely determined by the neamine core (ring I and II), which sits deep in the major groove and has both electrostatic and hydrogen bonding interactions.

The aminoglycoside-aptamer interactions were also predicted computationally with the NMR-derived aptamer structure as a rigid body to dock each of 9 aminoglycosides. Although most available docking software programs have been developed for protein-ligand systems, use of the docking software DOCK for a nucleic acid-ligand system was validated with a set of available RNA-small complexes previously solved by X-ray crystallography. After some modification of the algorithms and parameters, $70 \%$ of the small molecules with fewer than seven rotatable bonds and $26 \%$ of the small molecules with fewer than 13 rotatable bonds could be successfully recreated (Lang et al. 2009). Here, the experimental results from ITC analysis of aminoglycoside binding correlated well with the molecular docking scores $\left(R^{2}=0.71\right)$, which suggests that the NMR structure determined with neomycin-B in the aptamer pocket (Jiang et al. 1999) is similar to that which interacts with other aminoglycosides. One exception was ribostamycin.

By comparison with the reported high specificity of the neomycin aptamer for neomycin-B, we found a broader specificity for a number of aminoglycosides. To understand the molecular basis for the specificity, we simulated the occupied and

unoccupied aptamer using MD simulations. The simulated occupied aptamer was within $3 \AA$ RMSD of the initial NMR structure and so appeared essentially the same as the NMR structure. With the neomycin-B removed from the NMR coordinates, the aptamer retained 
essentially the same structure but the adenosine at position 16 of the pentaloop was extremely mobile. This base was predicted by the MD simulation of the occupied aptamer to interact with the nonpolar face of the fourth neomycin-B ring. A similar result was predicted computationally by Ligplot.

We tested the predictions of the MD simulations regarding the interaction of A16 with aminoglycosides by replacing A16 with 2-aminopurine. The resulting aptamer (2AP16) demonstrated an increase in fluorescence on binding to aminoglycosides, which is expected if the interaction changes the position of A16 to a more solvent accessible location. This result confirmed the Ligplot showing the hydrophobic interaction and MD predictions where we found it flexible as it transits from bound to unbound form. We then calculated the $\mathrm{K}_{d} \mathrm{~S}$ for neomycin-B, paramomycin and ribostamycin using the 2AP fluorescence change as monitor of binding. The results from these studies matched the estimated $\mathrm{K}_{\mathrm{d}} \mathrm{S}$ obtained by ITC for neomycin-B and paromomycin but the results for ribostamycin were quite different. As ribostamycin was the only aminoglycoside for which the correlation was very poor between ITC and DOCK scores, it was of interest to note that the $\triangle G$ determined for ribostamycin based on the 2AP titration was much more in line with the other aminoglycosides. These results suggest that the interaction between the aptamer and ribostamycin might involve a larger entropic component than for the other aminoglycosides, which appear to be mainly enthalpy-driven resulting from electrostatic and hydrogen bonding interactions as evidenced by the negative $\Delta \mathrm{H}$ and $\Delta \mathrm{S}$ determined by ITC.

We explored the basis for the very different reported specificities of the neomycin-B aptamer and discovered that the interaction of this aptamer with aminoglycosides is very dependent on the buffer constituents. The buffer in which all of our earlier experiments were performed contained a mixture of salts at the concentration expected inside the average mammalian cell with an appropriate intracellular $\mathrm{pH}$. In this buffer, and in the phosphate 
buffer used for obtaining the NMR structure, the aptamer shows a broad specificity with less preference for neomycin-B over paromomycin than it does in the cacocylate-based buffer used in previous studies (Wallis et al. 1995). These results were obtained using the fluorescent output of the 2AP16 aptamer. When similar results were sought by ITC, it was found that the titration in the presence of the NMR buffer demonstrated evidence of a structural change in the form of a significant increase in heat release in the initial phase of the titration followed by a standard titration profile. We examined the possibility that this dip in the ITC titration was related to the $\mathrm{pH}$ or lack of divalent cation content of the buffer and identified the latter as the reason. When magnesium was included in the buffer, the initial dip in the titration profile was largely eliminated. Magnesium ions are known to stabilize RNA tertiary structure (Misra and Draper 1998). Although previously thought to promote RNA folding by shielding the electrostatic repulsion between the RNA backbone charges, a recent analysis suggests that the role of magnesium is to facilitate counterion uptake, decreasing its entropic penalty and thereby reducing disorder of the ensemble (Fiore et al. 2012).

Our results suggest that the presence of magnesium increases the adoption of a structure compatible with high affinity aminoglycoside binding, but it does not affect the specificity of the aptamer for aminoglycosides. This is evident when the relative affinities for the aminoglycosides are compared in $10 \mathrm{mM}$ phosphate buffer in the presence and absence of $5 \mathrm{mM} \mathrm{MgCl} 2$ and also in the more complex buffer $\mathrm{A}$ that mimics intracellular conditions. On the other hand, cacodylate buffer $(\mathrm{E})$ that contains $\mathrm{NaCl}, \mathrm{KCl}$ and $\mathrm{NH}_{4} \mathrm{Cl}$ has a dramatic effect on aptamer specificity.

We also examined the role of adenosine 16 in the pentaloop and discovered that this base does not contribute to binding of neomycin-B and can be deleted without changing the affinity of the aptamer for this aminoglycoside. This result was surprising because the 
reported NMR structure shows the base lying over the sugar and interacting by hydrogen bonding. Unlike for neomycin-B, the aptamer affinity for ribostamycin was very sensitive to both the presence and nature of the base in position 16. Another profile was observed for the binding of paromomycin for which the nature of the base did not seem to be critical, but removing it decreased the binding affinity by 5 -fold. Thus, the nature of the interaction with the neomycin-B aptamer varies with the aminoglycoside and the specificity for aminoglycoside can be significantly altered by the buffer components in the environment and the change or removal of a single base.

\section{ACKNOWLEDGEMENTS}

This research is supported by the U.S. Department of Energy, Office of Biological and Environmental Research through the Ames Laboratory. The Ames Laboratory is operated for the U.S. Department of Energy by lowa State University under Contract No. DE-AC02-07CH11358. We thank Dr. Gulden Camci-Unal and Dr. Muneera Beach for their technical support in ITC use and data analysis and Dr. Pooja Arora for help in using GAMMES22. Also, we would like to thank the members of MNH lab, Dr. Tianjiao Wang and Lee Bendickson for their helpful discussions and technical support. 


\section{REFERENCES}

Al-Hashimi H M and Walter N G (2008). RNA dynamics: it is about time. Curr Opin Struct Biol 18(3): 321-329.

Chia J S, Wu H L, Wang H W, Chen D S and Chen P J (1997). Inhibition of hepatitis delta virus genomic ribozyme self-cleavage by aminoglycosides. J Biomed Sci 4: 208-216.

Cowan J A, Ohyama T, Wang D and Natarajan K (2000). Recognition of a cognate RNA aptamer by neomycin B: quantitative evaluation of hydrogen bonding and electrostatic interactions. Nucleic Acids Res 28(15): 2935-2942.

Dahlberg A E (1989). The functional role of ribosomal RNA in protein synthesis. Cell 57(4): 525-529.

de-los-Santos-Alvarez N, Lobo-Castanon M J, Miranda-Ordieres A J and Tunon-Blanco P (2007). Modified-RNA aptamer-based sensor for competitive impedimetric assay of neomycin B. J Am Chem Soc 129(13): 3808-3809.

de-los-Santos-Alvarez N, Lobo-Castanon M J, Miranda-Ordieres A J and Tunon-Blanco P (2009). SPR sensing of small molecules with modified RNA aptamers: detection of neomycin B. Biosens Bioelectron 24(8): 2547-2553.

Fiore J L, Holmstrom E D and Nesbitt D J (2012). Entropic origin of Mg2+-facilitated RNA folding. Proceedings of the National Academy of Sciences 109(8): 2902-2907.

Forster U, Weigand J E, Trojanowski P, Suess B and Wachtveitl J (2012). Conformational dynamics of the tetracycline-binding aptamer. Nucleic Acids Res 40(4): 1807-1817.

Jiang L, Majumdar A, Hu W, Jaishree T J, Xu W and Patel D J (1999). Saccharide-RNA recognition in a complex formed between neomycin B and an RNA aptamer. Structure 7(7): 817-827.

Kuntz I D, Blaney J M, Oatley S J, Langridge R and Ferrin T E (1982). A geometric approach to macromolecule-ligand interactions. J Mol Biol 161(2): 269-288.

Kwon M, Chun S M, Jeong S and Yu J (2001). In vitro selection of RNA against kanamycin B. Mol Cells 11(3): 303-311.

Lang P T, Brozell S R, Mukherjee S, Pettersen E F, Meng E C, Thomas V, Rizzo R C, Case D A, James T L and Kuntz I D (2009). DOCK 6: Combining techniques to model RNA-small molecule complexes. RNA 15(6): 1219-1230.

Lato S M, Boles A R and Ellington A D (1995). In vitro selection of RNA lectins: using combinatorial chemistry to interpret ribozyme evolution. Chem Biol 2: 291-303. 
Lee J, Kwon M, Lee K H, Jeong S, Hyun S, Shin K J and Yu J (2004). An approach to enhance specificity against RNA targets using heteroconjugates of aminoglycosides and chloramphenicol (or linezolid). J Am Chem Soc 126(7): 1956-1957.

Mikkelsen N E, Brannvall M, Virtanen A and Kirsebom L A (1999). Inhibition of RNase P RNA cleavage by aminoglycosides. Proc Natl Acad Sci USA 96: 6155-6160.

Mikkelsen N E, Johansson K, Virtanen A and Kirsebom L A (2001). Aminoglycoside binding displaces a divalent metal ion in a tRNA-neomycin B complex. Nat Struct Biol 8(6): 510-514.

Misra V K and Draper D E (1998). On the role of magnesium ions in RNA stability. Biopolymers 48(2-3): 113-135.

Moazed D and Noller H F (1987). Interaction of antibiotics with functional sites in $16 S$ ribosomal RNA. Nature 327: 389-394.

Nick Taylor J, Darugar Q, Kourentzi K, Willson R C and Landes C F (2008). Dynamics of an anti-VEGF DNA aptamer: a single-molecule study. Biochem Biophys Res Commun 373(2): 213-218.

Pettersen E F, Goddard T D, Huang C C, Couch G S, Greenblatt D M, Meng E C and Ferrin T E (2004). UCSF Chimera--a visualization system for exploratory research and analysis. J Comput Chem 25(13): 1605-1612.

Rogers J, Chang A H, von Ahsen U, Schroeder R and Davies J (1996). Inhibition of the selfcleavage reaction of the human hepatitis delta virus ribozyme by antibiotics. $\mathrm{J}$ Mol Biol 259: 916-925.

Spackman M A (1996). Potential derived charges using a geodesic point selection scheme. Journal of Computational Chemistry 17(1): 1-18.

Stage T K, Hertel K J and Uhlenbeck O C (1995). Inhibition of the hammerhead ribozyme by neomycin. Rna 1(1): 95-101.

Stampfl S, Lempradl A, Koehler G and Schroeder R (2007). Monovalent ion dependence of neomycin B binding to an RNA aptamer characterized by spectroscopic methods. Chembiochem 8(10): 1137-1145.

Tok J B, Cho J and Rando R R (1999). Aminoglycoside antibiotics are able to specifically bind the 5'-untranslated region of thymidylate synthase messenger RNA. Biochemistry 38(1): 199-206.

Tor Y, Hermann T and Westhof E (1998). Deciphering RNA recognition: aminoglycoside binding to the hammerhead ribozyme. Chem Biol 5(11): R277-283. 
von Ahsen U, Davies J and Schroeder R (1991). Antibiotic inhibition of group I ribozyme function. Nature 353: 368-370.

von Ahsen U, Davies J and Schroeder R (1992). Non-competitive inhibition of group I intron RNA self-splicing by aminoglycoside antibiotics. J Mol Biol 226: 935-941.

Wallace A C, Laskowski R A and Thornton J M (1995). LIGPLOT: a program to generate schematic diagrams of protein-ligand interactions. Protein Eng 8(2): 127-134.

Wallis M G and Schroeder R (1997). The binding of antibiotics to RNA. Prog Biophys Mol Biol 67: 141-154.

Wallis M G, von Ahsen U, Schroeder R and Famulok M (1995). A novel RNA motif for neomycin recognition. Chem Biol 2: 543-552.

Wang S, Huber P W, Cui M, Czarnik A W and Mei H Y (1998). Binding of neomycin to the TAR element of HIV-1 RNA induces dissociation of Tat protein by an allosteric mechanism. Biochemistry 37(16): 5549-5557.

Wang Y, Hamasaki K and Rando R R (1997). Specificity of aminoglycoside binding to RNA constructs derived from the 16S rRNA decoding region and the HIV-RRE activator region. Biochemistry 36(4): 768-779.

Wang Y, Killian J, Hamasaki K and Rando R R (1996). RNA Molecules That Specifically and Stoichiometrically Bind Aminoglycoside Antibiotics with High Affinities. Biochemistry 35(38): 12338-12346.

Wang $Y$ and Rando R R (1995). Specific binding of aminoglycoside antibiotics to RNA. Chem Biol 2: 281-290.

Werstuck G and Green M R (1998). Controlling gene expression in living cells through small molecule-RNA interactions. Science 282(5387): 296-298.

Zapp M L, Stern S and Green M R (1993). Small molecules that selectively block RNA binding of HIV-1 Rev protein inhibit Rev function and viral production. Cell 74(6): 969-978.

Zoete V, Cuendet M A, Grosdidier A and Michielin O (2011). SwissParam: a fast force field generation tool for small organic molecules. J Comput Chem 32(11): 2359-2368. 


\section{FIGURE LEGENDS}

Figure 1. Chemical structures of neomycin and kanamycin-class aminoglycosides used in docking with 23mer neomycin-B RNA aptamer and comparison of docking scores with experimental results. A) The neomycin-class aminoglycoside antibiotics have a 2-deoxystreptamine (2-DOS, ring II), disubstituted at positions 4 and 5 positions while the kanamycin-class has substitutions at positions 4 and 6 . The substitutions at the $\mathrm{R}$ positions are indicated for neomycin-class aminoglycoides. For kanamycin-class aminoglycosides, the main substations (R1 and R2) are located on the ring I. Sisosmicin and netilmicin differ with only a single substitution at the $\mathrm{R}$ position on 2-DOS (ring II). The structure of geneticin differ from the rest of the aminoglycosides such that it has an extra methyl attached to C6 on the ring $\mathrm{I}$, and the other two attached to ring $\mathrm{III}$ one on the amino at $3^{\text {rd }}$ position and the other at $4^{\text {th }}$ position. B) Predicted secondary structure of the 23mer Neomycin-B RNA Aptamer. The secondary structure of the neomycin-B RNA aptamer was predicted by RNAstructure4.6 (Matthews et al. 2004). In order to be consistent with the previous NMR study, we kept the same numbering used in Jiang et al. (1999) in which the first base is labeled as position 4. C) Comparison of the predicted docking scores obtained from DOCK6.2 with the experimentally (ITC)-calculated binding free energies $(\Delta \mathrm{G})$ of aminoglycosides binding to the neomycin-B RNA aptamer Isothermal titration calorimetry experiments determined the binding free energies $(\mathrm{kcal} / \mathrm{mol})$ for aminoglycosides as follows: neomycin-B < ribostamycin < kanamycin-B < paromomycin < tobramycin $<$ sisomicin $<$ gentamicin < geneticin < kanamycin-A. This is the order of binding from the strongest to the weakest binder of the aptamer. Also, docking predicted the order of aminoglycoside binding with $71 \%$ correlation with ITC experiments if ribostamycin were not included. With ribostamycin (gray triangle) included in the calculation the correlation was $36 \%$. 
Figure 2. Comparison of RMSDs from molecular dynamics (MD) simulations. Comparison of the RMSDs between the occupied (black) and unoccupied (gray) MD simulations showed the A16 as the most flexible segment and with the largest difference between occupied and unoccupied forms in the region of $\mathrm{A} 14$ to $\mathrm{A} 16$. In the occupied aptamer the A16 is found over the neomycin-B. The corresponding atom numbers for the residue A16 are located between 395 and 427, where the highest difference in RMSD is observed between the occupied and unoccupied RNA simulations.

Figure 3. Aptamer affinity monitored by 2AP fluorescence and compared with ITC. A) The 2AP16 aptamer was titrated with neomycin-B (black squares), paromomycin (gray circles) and ribostamycin (white triangles) and the titration curves were fitted for 2AP fluorescence using a hyperbolic model. B) The Ka's were determined by ITC (black bars) and 2AP fluorescence (gray bars). Neomycin-B and paromomycin binding affinities are similar when calculated using either method (fluorescence or ITC); however, the ribostamycin binding affinity differed depending on the method. C) When the $\mathrm{Ka}$ for ribostamycin from 2AP fluorescence $(\mathrm{FI})$ was included in figure $1 \mathrm{C}$, the ribostamycin point is closer to the correlation fit line obtained from the comparison of ITC and docking scores. Figure 4. Effect of buffer on aminoglycoside binding to the neomycin-B aptamer. A comparison of 2AP fluorescence changes upon neomycin-B (black squares), paromomycin (gray circles) and ribostamycin (white triangles) binding in the presence of different ionic conditions. Buffer B: NMR buffer at $\mathrm{pH}$ 6.1, Buffer C: NMR buffer at pH 7.3, Buffer D: NMR buffer with $5 \mathrm{mM} \mathrm{MgCl} 2$ at $\mathrm{pH} 7.3$ and Buffer E: in cacodylate-based binding buffer used by Wallis et al. In buffer $\mathrm{E}$, the binding of paromomycin and ribostamycin is significantly lower than in other buffers, whereas the binding of neomycin-B is not affected. The fluorescence measurements were taken at $370 \mathrm{~nm}$ after exciting at $307 \mathrm{~nm}$ in a spectrofluorometer. 
Figure 5. Comparison of heat releases upon aminoglycoside binding to neomycin RNA aptamer in buffers with varying $\mathrm{pH}$ or $\mathbf{M g}$. A) The effect of $\mathrm{pH}$ on heat release upon neomycin-B and paromomycin binding at $\mathrm{pH} 6.1$ (gray) and at $\mathrm{pH} 7.3$ (black). The binding modes for neomycin-B and paromomycin were the same at different pHs. However, the observed heat releases were larger at $\mathrm{pH} 7.3$ for both titrations. B) The heat releases from neomycin-B, paromomycin and ribostamycin binding with (gray) and without magnesium ions (black). With the inclusion of magnesium the initial dip in the heat release was not observed.

Figure 6. The effect of adenine on aminoglycoside binding and selectivity of the neomycin-B RNA aptamer. Replacing the adenine with other nucleotide bases (cytosineA16C, guanine-A16G and uracil-A16C) or removing $(\Delta \mathrm{A} 16)$ changes the behavior of the neomycin-B RNA aptamer in terms of its selectivity and ligand binding ability in buffer A. The data represent the average association constants $\left(\mathrm{K}_{a} \mathrm{~S}\right)$ obtained from a minimum of two replicate ITC experiments. Refer to the supplemental Table 4 for the $K_{d}$ values for each variant. 


\section{FIGURES AND TABLES}

\section{Figure 1}
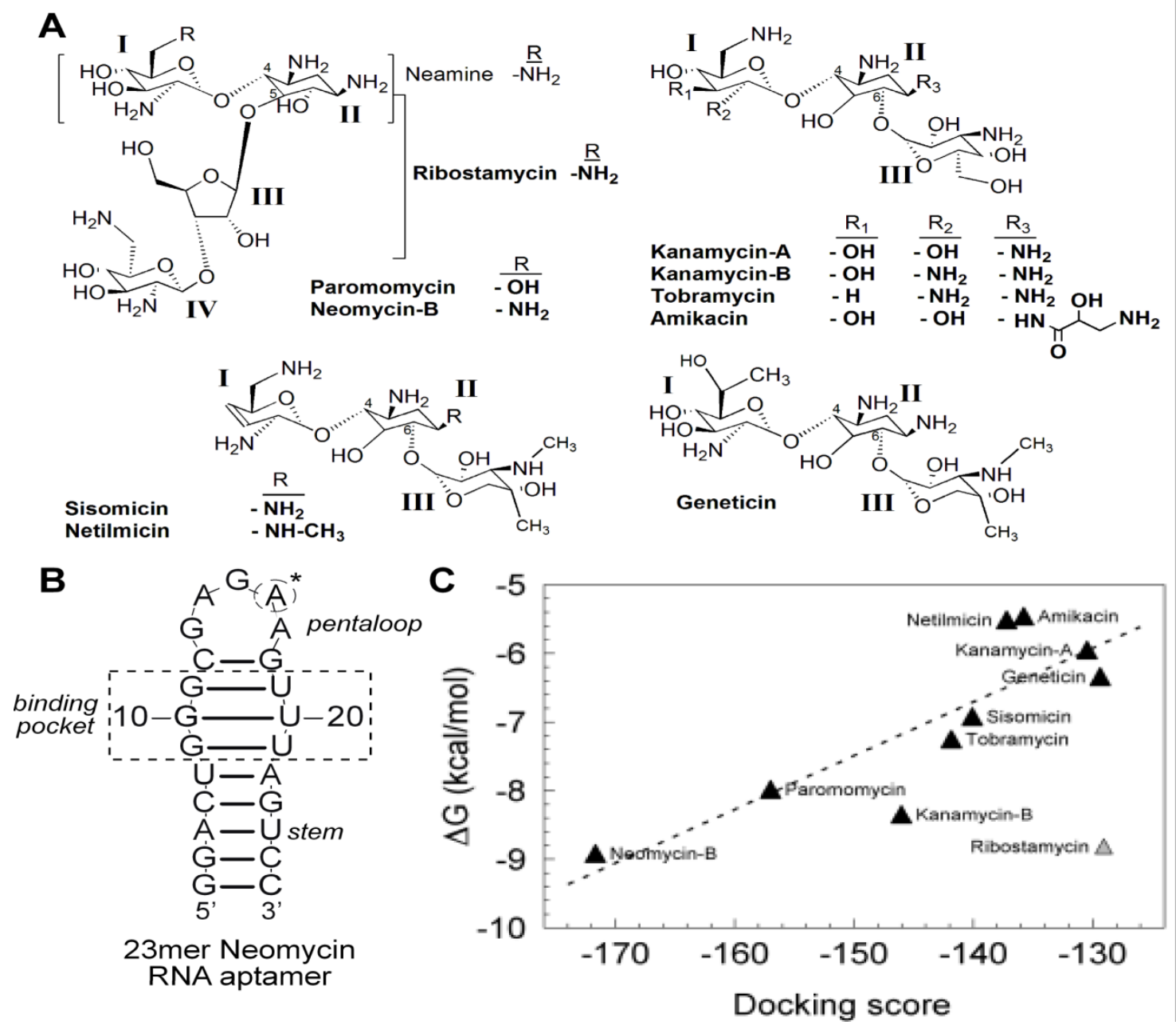
Figure 2
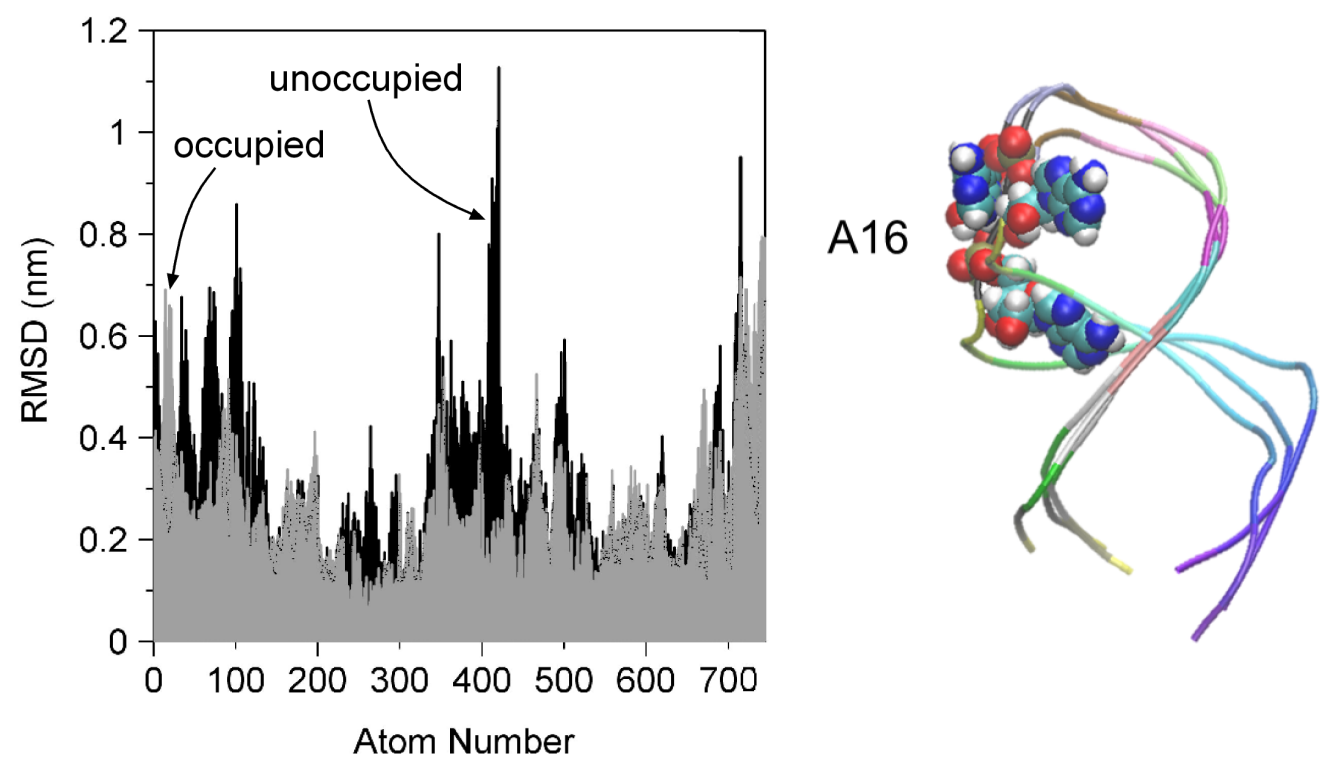
Figure 3

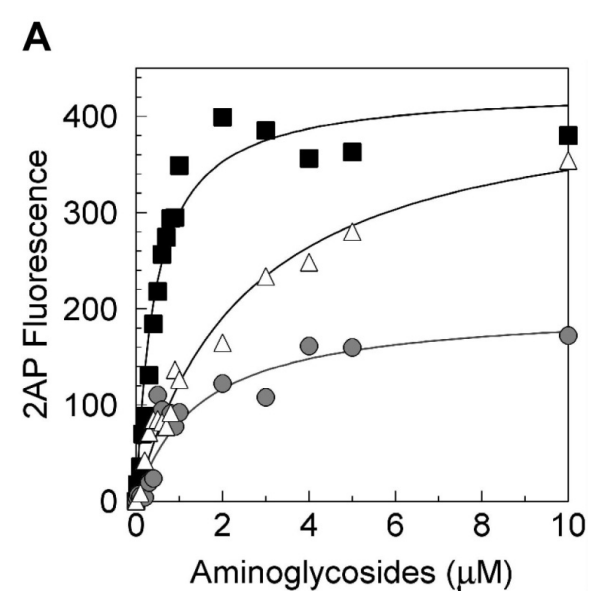

B $\mathrm{Ka}\left(\mathrm{x} 10^{-6}\right)$

C

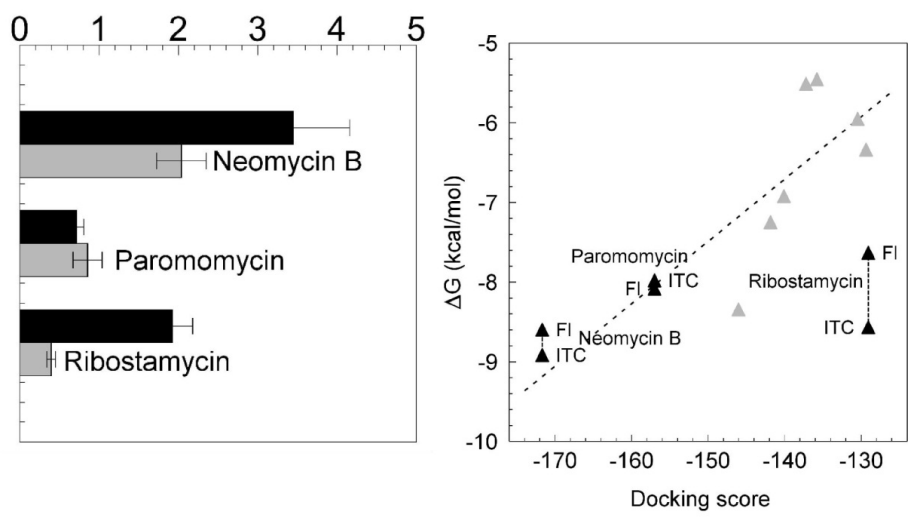

Figure 4
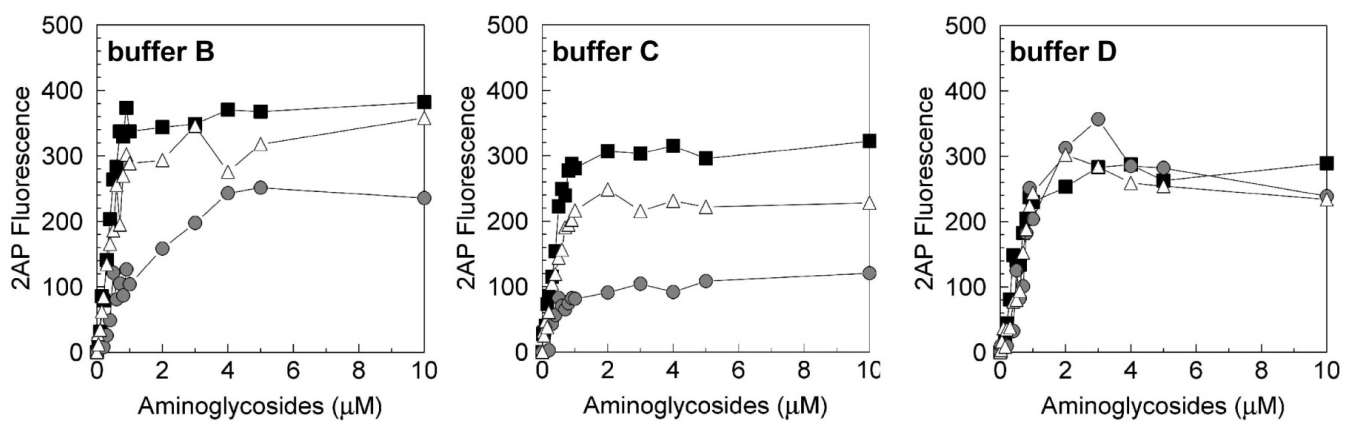

\begin{tabular}{|c|c|c|c|c|c|c|c|}
\hline \multirow{2}{*}{ buffer } & \multicolumn{5}{|c|}{ ions } & \multirow{2}{*}{\begin{tabular}{|c|} 
organic \\
cacodylate
\end{tabular}} & \multirow{2}{*}{$\mathrm{pH}$} \\
\hline & $\mathrm{K}^{+}$ & $\mathrm{Na}$ & ${ }^{+} \mathrm{NH}_{4}^{+}$ & $\mathrm{Mg}^{2}$ & $\mathrm{PO}_{4}^{3-}$ & & \\
\hline B & & 10 & & & 10 & & 6.1 \\
\hline C & & 10 & & & 10 & & 7.3 \\
\hline D & & 10 & & 5 & 10 & & 7.3 \\
\hline$E$ & 80 & 80 & 200 & 5 & & 80 & 7.4 \\
\hline
\end{tabular}

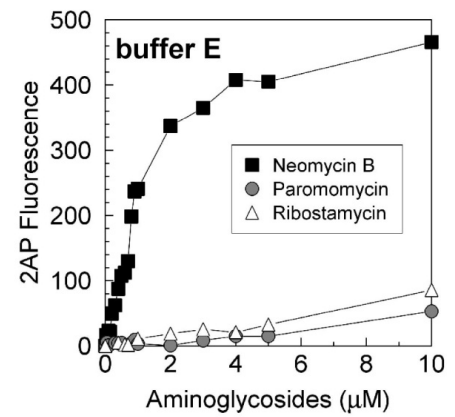


Figure 5

\section{pH effect}

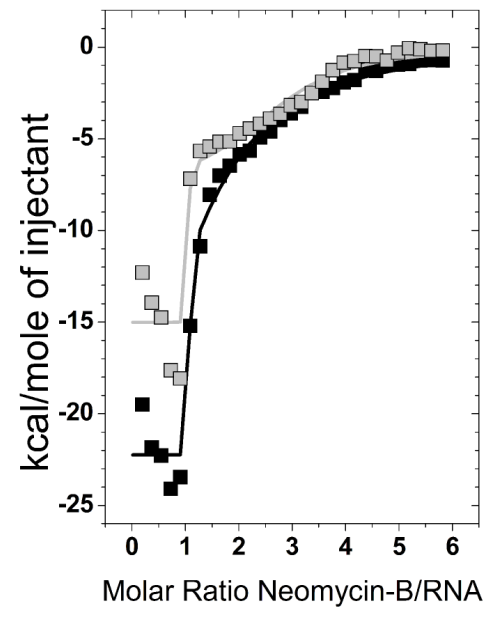

Magnesium effect

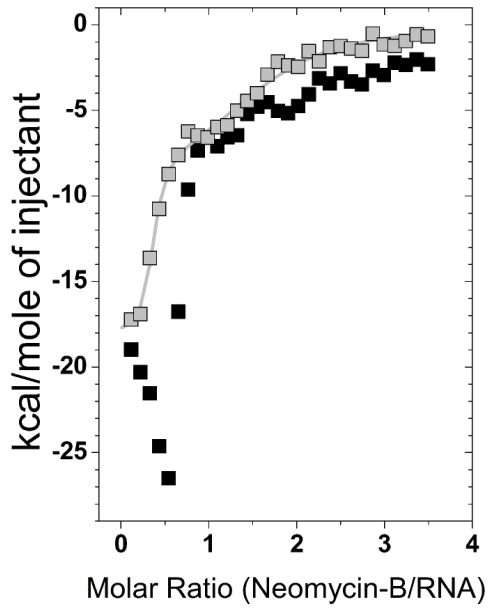

pH $6.1 \quad$ pH 7.3

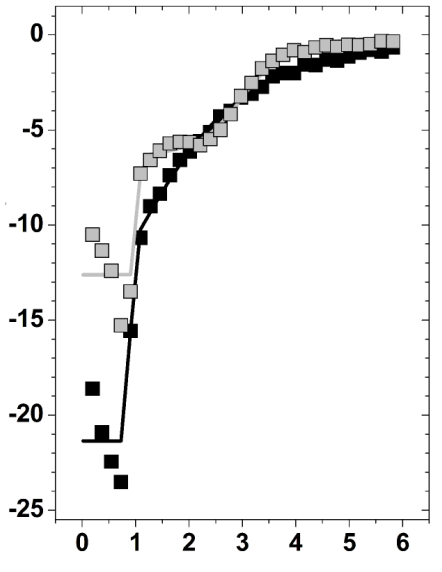

Molar Ratio (Paromomycin/RNA)

\begin{tabular}{|c|c|c|}
\hline buffer & $\mathbf{M g}$ & $\mathbf{p H}$ \\
\hline B & & 6.1 \\
\hline C & & 7.3 \\
\hline E & 5 & 7.3 \\
\hline
\end{tabular}

$10 \mathrm{mM} \mathrm{NaPO}_{4}$

$5 \mathrm{mM} \mathrm{Mg}$ no $\mathbf{M g}$
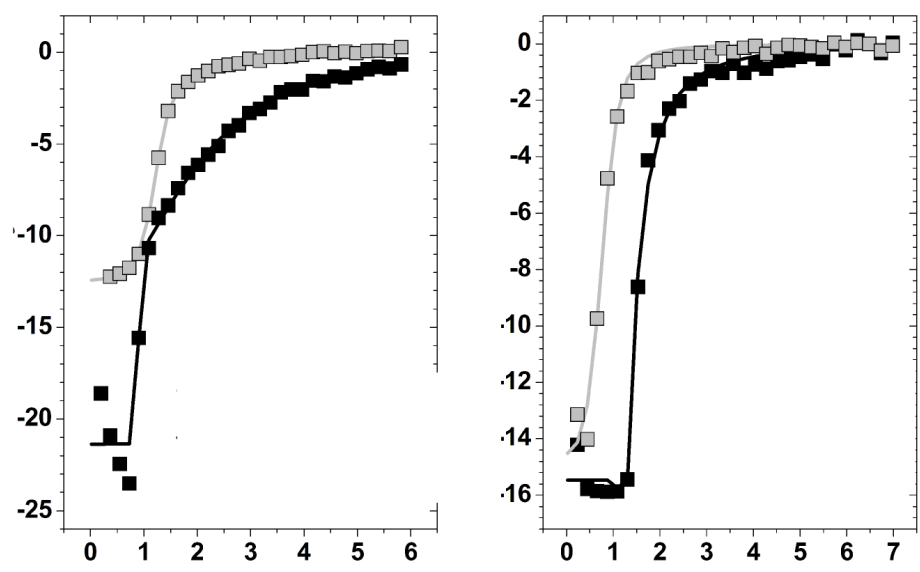

Molar Ratio (Ribostamycin/RNA) 
Figure 6

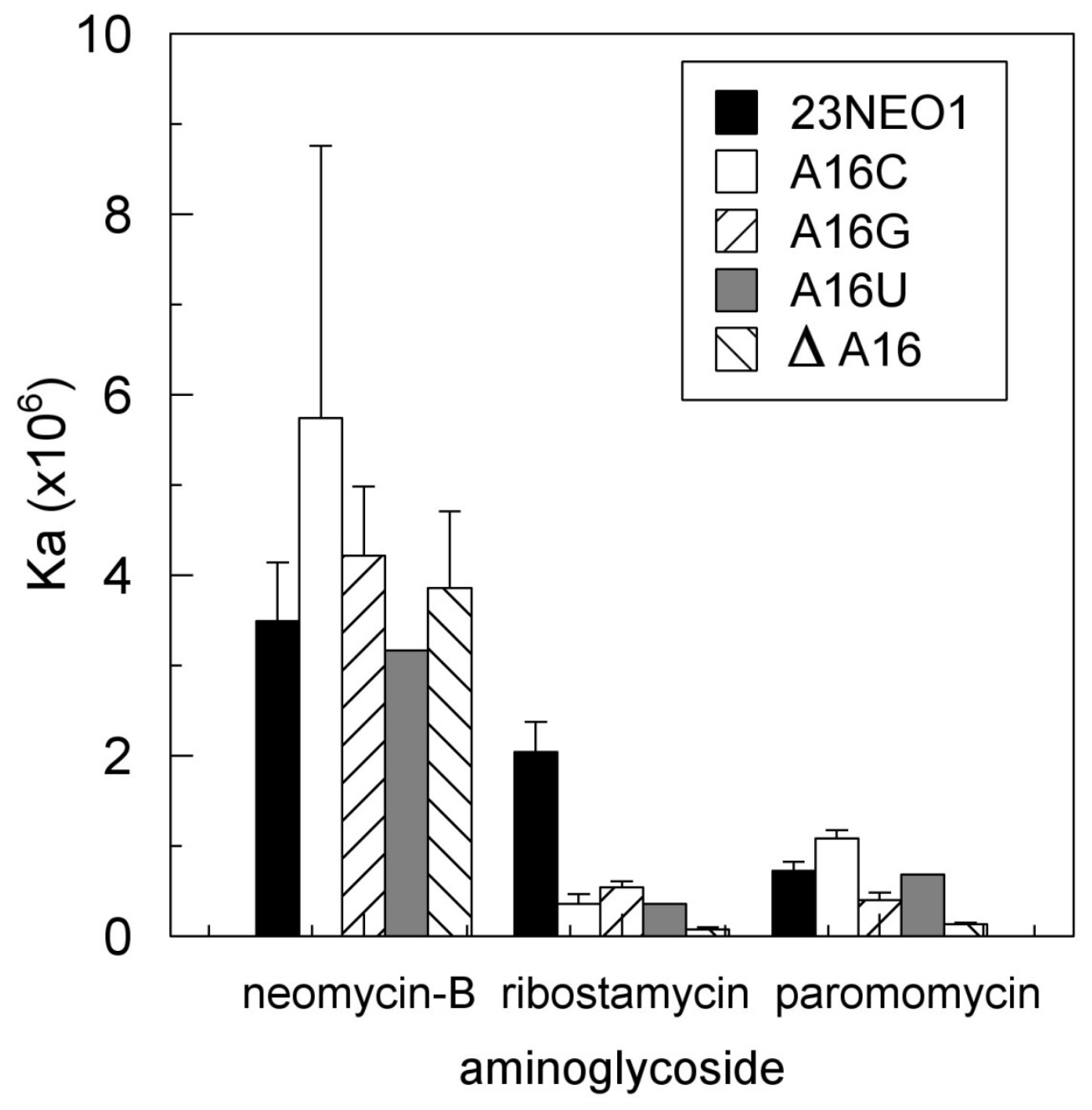




\section{SUPPORTING INFORMATION}

Table S1. Comparison of binding affinities of the neomycin-B RNA aptamer to a variety of aminoglycosides

\begin{tabular}{cc}
\hline & Kd, $\boldsymbol{\mu} \mathbf{M}$ (Replicates) \\
\hline Neomycin-B & $0.29 \pm 0.05(8)$ \\
Ribostamycin & $0.49 \pm 0.08(5)$ \\
Kanamycin-B & $0.75 \pm 0.26(5)$ \\
Paromomycin & $1.4 \pm 0.19(5)$ \\
Tobramycin & $4.8 \pm 0.95(3)$ \\
Sisomicin & $8.4 \pm 2.9(3)$ \\
Geneticin & $26 \pm 9.3(5)$ \\
Kanamycin-A & $43 \pm 3.6(3)$ \\
Amikacin & $90\left(4,1^{*}\right)$ \\
Netilmicin & $99\left(5,1^{*}\right)$ \\
\hline
\end{tabular}

Dissociation constants of aminoglycosides were determined via isothermal titration calorimetry (ITC) experiments as described in materials and methods section and their respective average $\mathrm{Kd}$ values are represented. *For amikacin and netilmicin, the value represent a single successful ITC result with a good fit out of 4 and 5 respective experiments, the rest indicated no observable interaction with the neomycin-B RNA aptamer. 
Table S2. Docking scores for the aminoglycosides

\begin{tabular}{lc}
\hline Ligand name & $\begin{array}{c}\text { DOCK score } \\
\text { (kcal/mol) for } \\
\text { explicitly protonated }\end{array}$ \\
\hline Neomycin-B & -171.678558 \\
Paromomycin & -156.995514 \\
Kanamycin-B & -147.330307 \\
Tobramycin & -141.835739 \\
Sisomicin & -139.940292 \\
Netilmicin & -138.805573 \\
Amikacin & -135.783234 \\
Kanamycin-A & -130.461761 \\
Geneticin & -129.358246 \\
Ribostamycin & -126.219162 \\
\hline
\end{tabular}

Docking scores obtained from DOCK6.2 and the scores are placed from the best binder to the least. 
Figure S3. Predicted interaction between the neomycin RNA aptamer and neomycinB via docking

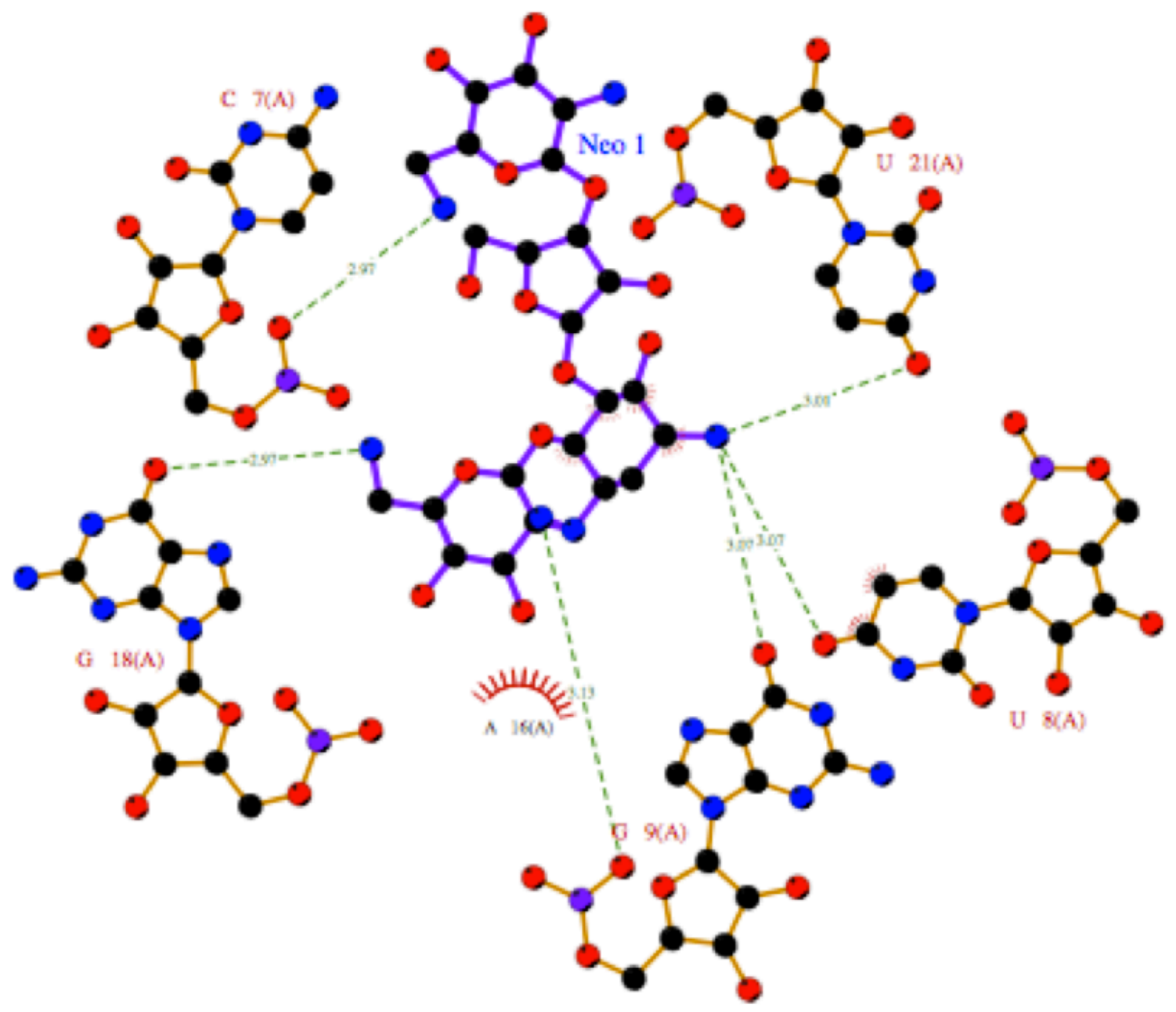

Key

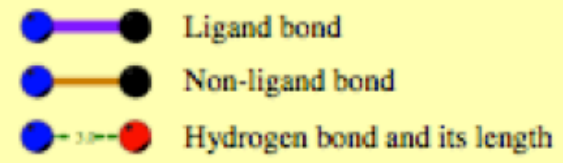

His 53 Non-ligand residues involved in hydrophobic $3 \pi$ contact(s)

Corresponding atoms involved in hydrophobic contact(s)

A16 was found to have hydrophobic interactions instead of hydrogen bonding as seen in NMR. Also, C7, U8, G9, G18, and U21 were found to have hydrogen bonding interactions with neomycin-B. 
Table S4. Comparison of binding affinities of the neomycin-B RNA aptamer variants to neomycin-B, ribostamycin and paromomycin.

\begin{tabular}{lccccc}
\hline & \multicolumn{5}{c}{ Dissociation Constants $(\boldsymbol{\mu M})$} \\
\cline { 2 - 6 } Neomycin-B & \multicolumn{1}{c}{ A16 } & \multicolumn{1}{c}{ A16C } & A16U & A16G & $\Delta$ A16 \\
Ribostamycin & $0.49 \pm 0.05$ & $0.17 \pm 0.09$ & $0.32 \pm 0.1$ & $0.24 \pm 0.04$ & $0.26 \pm 0.06$ \\
Paromomycin & $1.4 \pm 0.19$ & $2.8 \pm 0.85$ & $2.8 \pm 0.19$ & $1.85 \pm 0.21$ & $13.3 \pm 3.89$ \\
\hline
\end{tabular}

Dissociation constants of aminoglycosides were determined via ITC experiments as described in materials and methods section with buffer $A$ and their respective average $K_{d}$ values are represented. 
Table S5. Dissociation constants of aminoglycosides to the neomycin aptamer in different buffers.

\begin{tabular}{cccccc}
\hline \multicolumn{5}{c}{ Dissociation Constants $(\mu \mathrm{M})$} \\
\hline & Buffer A & Buffer B & Buffer C & Buffer D & Buffer E \\
Neomycin-B & $0.53 \pm 0.09$ & $0.39 \pm 0.08$ & $0.39 \pm 0.06$ & $1.69 \pm 0.20$ & $0.79 \pm 0.23$ \\
Ribostamycin & $2.51 \pm 0.34$ & $0.45 \pm 0.08$ & $0.39 \pm 0.06$ & $24.9 \pm 8.51$ & $1.06 \pm 0.38$ \\
Paromomycin & $1.17 \pm 0.25$ & $1.21 \pm 0.25$ & $19.9 \pm 8.1$ & $23.7 \pm 11.3$ & $0.59 \pm 0.11$ \\
\hline
\end{tabular}

Comparison of the $\mathrm{K}_{d} \mathrm{~S}$ for neomycin- $\mathrm{B}$, paromomycin and ribostamycin binding under different buffer conditions calculated from the 2AP study. Aminoglycoside binding in buffer $\mathrm{A}, \mathrm{B}, \mathrm{C}, \mathrm{D}$ and $\mathrm{E}$. The fluorescence measurements are taken at $370 \mathrm{~nm}$ after exciting at $307 \mathrm{~nm}$ in a spectrofluorometer. 


\section{CHAPTER 4}

\section{DRAGINS: DRUG BINDING APTAMERS FOR GROWING INTRACELLULAR NUMBERS}

Muslum Ilgu, Supipi Liyamalli Auwardt, Robert Feldges, and Marit Nilsen-Hamilton*

${ }^{*}$ Corresponding author 


\begin{abstract}
One of the most challenging requirements in cancer treatment is maintaining sufficiently high intracellular drug concentration due to the upregulation of multi-drug transporters by cancer cells. Modern drug synthesis research has attempted to address this problem by many approaches that are mainly based on "push" mechanisms. However, the extent to which the extracellular drug concentration can be increased is limited. Therefore, we have tested a different approach based on a "pull" mechanism by expressing molecules that can bind drugs inside the cells. These expressed molecules are aptamers, single stranded nucleic acids selected for tight and specific binding to their targets. The action of mobile aptamers with specificity for a particular drug was predicted by a prior mathematical model to increase the intracellular drug concentration. To test this hypothesis, the $E$. coli BL21 strain was transformed with plasmids from which aptamers were expressed and the effect was observed over a $12 \mathrm{~h}$ period on the toxicity of a variety of aminoglycosides as compared to the E. coli without aptamers. In this study, we observed a decrease in the IC50 values by 2 to 5 fold for several aminoglycoside antibiotics with several aminoglycosidebinding aptamers. This study points to a completely new approach to increase the efficacy of drugs via intracellular expression of RNA aptamers or other small mobile drug receptors.
\end{abstract}




\section{INTRODUCTION}

The entry of unnatural chemicals or toxins into cells is limited by the lipid bilayer membrane that surrounds cells and serves as a protective layer. Most drugs used to treat diseases must penetrate this barrier to reach their targets without the benefit of transporters that are used by cells to import natural products for metabolism. Cells have mechanisms to increase their survival rates in the presence of toxins. Cancer cell populations evolve to upregulate the drug transporters or increase expression of the target protein of the drug and thereby become drug resistant. In either case, the drug cannot accumulate to a sufficiently high concentration inside the cells, which as a consequence are more likely to survive and proliferate. Cancer cells that survive a drug treatment frequently have elevated levels of the drug pumps called Pgp (P-glycoprotein) or MDR (multi drug resistance protein) [1, 2]. These efflux pumps help the cells in which they are elevated to survive, with the result that the cancer cell population tends to become increasingly drug resistant with continued drug treatment. Cells can also become resistant to a drug by increasing the intracellular concentration of the drug's target protein. The excess target protein soaks up the drug, leaving some of the target protein still active and thus these cells survive.

Many approaches have been taken to combat drug resistance [3]. These approaches involve either directly targeting the pumps with drugs or antibodies, or decreasing the pump mRNA levels by antisense DNAs or RNAi, or decreasing pump mRNA production by targeting the relevant transcriptional regulators. Computational approaches can also be used to design anti-pump drugs and recently high-resolution 3D structures of specific protein pumps supported these approaches. One of these strategies may eventually lead to the development of small molecule drugs to solve the problem of multi drug resistance; however, none of them have so far been very successful in combating multi 
drug resistance a new approach to combating the resistant condition would have useful medical applications.

By mathematical modeling it was proposed that the intracellular concentration of drug might be increased by the presence of a receptor for that drug inside a mammalian cell [4]. In that study, the receptor was proposed to be a small RNA aptamer that can bind to a drug. Aptamers were also utilized as intramers in several other studies and successfully shown to be effective in inhibiting their targets [5,6] or controlling gene expression [7]. Here, we tested, in a bacterial system, the effects of expressed the RNA aptamers that could bind to aminoglycosides on the IC50 values of the aminoglycosides. Our hypothesis was that aminoglycoside-RNA-binders, when expressed in bacteria, will increase the sensitivity of the bacteria to the aminoglycoside to which the aptamer bound. We used both natural RNAs and in vitro selected aptamers that bind aminoglycosides. The complete binding affinities of a group of aminoglycosides to these RNAs were determined. The RNAs were cloned for bacterial expression and tested for their effects on bacterial growth in the presence and absence of aminoglycosides over a $12 \mathrm{~h}$ period. The results showed that expression of several aminoglycoside-binding aptamers lowered the IC 50 values by 2 to 5 fold for the aptamer aminoglycoside ligands.

Currently, we are testing the other predictions from the mathematical model [4]. One prediction was that expressing aptamers would increase the intracellular concentration of the target drug. Therefore, we are developing a method to measure the intracellular concentration of aminoglycosides. Another prediction to test is the mobility of the aptamers, which was shown to be critical for the aptamer effect on drug concentration. To test this, aptamers will be cloned into a larger mRNA cassette and the effect of these longer RNAs compared with shorter single unit aptamers. 


\section{Mechanism of action of the aminoglycosides}

Aminoglycoside antibiotics are produced as secondary metabolites and secreted by Streptomyces and Actinomyces spp. to have advantage over its neighboring species. These natural compounds were isolated from their fungal sources and have been successfully used as antibiotics for many years. In addition, there are semi-synthetic aminoglycosides like amikacin, dibekacin, sisomicin and netilmicin. Chemically, aminoglycosides can be divided into two main categories; streptomycin and 2deoxystreptamine-containing ones. The latter is further divided into neomycin-class or 4,5disubstituted (e.g., neomycin-B, ribostamycin and paromomycin) and kanamycin-class or 4,6-disubstituted deoxystreptamine (e.g., tobramycin, kanamycin-A and -B) [8]. Both classes are composed of amino sugars linked to its basic building block, 2deoxystreptamine with common functional groups on rings I and II, which form the neamine core (Fig.1A).

Aminoglycosides interact directly with the conserved regions of the ribosomal RNA A-site (aminoacyl-tRNA site or decoding region) in the bacterial ribosomes as their primary target and exert their biological effects [9-12]. Aminoglycoside binding shifts the position of the two adenines A1492 and A1493 in the 16S rRNA and decreases the dissociation rate of tRNAs from the A-site, thereby inducing misreading of the genetic code and inhibiting translation, which in turn results in bacterial cell death $[13,14]$. In addition, aminoglycosides' preferential targeting capability to prokaryotic ribosomes over eukaryotic ribosomes makes their clinical use more successful [15] and they have been used to treat Gram-negative and Gram-positive bacterial infections for over half a century [8]. 


\section{Possible RNAs to use as DRAGINs that interact with aminoglycosides}

A variety of small RNAs have been shown to interact with aminoglycosides over the past thirty years [16-18]. These RNAs include mainly non-coding RNAs whose function and activity can be biologically significant but are interfered with by the binding of aminoglycosides.

In addition to the A-site, other RNAs involved in a variety of biochemical pathways have been recognized as aminoglycoside targets. For example, aminoglycosides are also known to interact with viral mRNAs; Rev-responsive element (RRE) [19-21] and transactivating region (TAR) of HIV-1 [22, 23], ribozymes; the hammerhead [24, 25], hairpin [26] and human hepatitis delta virus (HDV) [27, 28]. Furthermore, yeast tRNA ${ }^{\text {Phe }}$ [29] and tRNA $^{\text {Asp }}[30]$, iron responsive element (IRE) [31], the T-box antiterminator RNA [32], the 5' untranslated region of thymidylate synthase mRNA [33], transfer messenger RNA (tmRNA) [34], RNase P [35], group I self-splicing intron [36-38] and the tau exon 10 splicing regulatory element [39] can also be targeted by aminoglycosides. In addition to these natural RNAs, some aminoglycoside-specific RNA aptamers have been in vitro selected against neomycin-B [40, 41], tobramycin [7, 42], kanamycin-A [7] and kanamycin-B [43] using SELEX. Studies of the interaction of these functional domains of RNAs with aminoglycosides have validated the potential use of aminoglycosides as successful drugs.

Aminoglycoside-RNA binding is mediated through hydrogen bonding between

amino and hydroxyl groups of aminoglycosides and RNA bases [42] as well as mutual electrostatic interactions between the positively charged amino groups of the aminoglycosides and negatively charged phosphate backbone of the RNA [44]. As a result, aminoglycosides can bind to the bulge regions of the RNAs listed above with affinities in the low $\mu \mathrm{M}$ range. We utilized the smallest aminoglycoside-binding RNAs including the 23 mer neomycin, 25mer kanamycin and 27mer tobramycin aptamers as well as 27 mer A-site, 
30mer RRE of HIV-1 and 33mer thymidylate synthase mRNA region in our ITC studies and observed their effects on decreasing bacterial growth as predicted by the previous mathematical model.

\section{MATERIALS AND METHODS}

Bacterial growth curves were determined in a Synergy2 plate-reader (Biotek). Nontissue culture treated, flat bottom 96-well polystyrene microtest plates were purchased from Falcon (originally from Becton-Dickinson Labware). 96-well plate sealing membranes were regular strength breathe-easy sealing membranes from Research Products International Corp. Micropipettes were Eppendorf purchased from Reference and Pipetmen were from Gilson. Test tubes were $14 \mathrm{ml}$ Polystyrene Round-Bottom Tubes from Falcon. The incubator was a Max-Q 4000 (Branstead Lab Inc.).

Aminoglycoside antibiotics used in the experiments were neomycin-B, paromomycin, tobramycin, kanamycin-A and -B that were obtained as their sulfate salts from Sigma-Aldrich (St. Louis, MO). E. coli BL21(DE3)star was obtained from Invitrogen (Eugene, OR) as chemically competent.

The list of plasmids is as follows.

pMU159 (empty plasmid used as a control); kanamycin aptamer; 1mer, 3mer, $7 \mathrm{mer}$, 12mer, 14mer and 28mer, tobramycin aptamer; $1 \mathrm{mer}$, 5mer, $7 \mathrm{mer}$, 8mer, 10mer, 12mer, neomycin aptamer; $1 \mathrm{mer}$, 3mer, 8mer, 15mer, 16mer, 24mer, 32mer. A-site mimic/decoding region; $1 \mathrm{mer}$ and 3mer, thymidylate synthase mRNA region; $1 \mathrm{mer}$ and $3 \mathrm{mer}$ and Revresponsive element (RRE); 1 mer and 2 mer.

All RNA oligonucleotides were obtained from Integrated DNA Technologies Inc. (Coralville, IA) and they were kept at $-20^{\circ} \mathrm{C}$ in $\mathrm{ddH}_{2} \mathrm{O}$ until used in the isothermal titration calorimetry (ITC) assays. 


\section{Bacterial growth analysis}

Transformed BL21 cultures were primarily grown for about $16 \mathrm{~h}$ at $37^{\circ} \mathrm{C}$ in LuriaBertani (LB) broth with $100 \mu \mathrm{g} / \mathrm{mL}$ ampicillin. Then, the cultures were diluted about 100 times and induced with $1 \mathrm{mM}$ IPTG for $1 \mathrm{~h}$ at $37^{\circ} \mathrm{C}$ with shaking $250 \mathrm{rpm}$. Following the induction, aminoglycosides (neomycin-B, paromomycin, tobramycin, and kanamycin-A and B) were added and the cultures were grown for $12 \mathrm{~h}$ at $37^{\circ} \mathrm{C}$ in a plate reader (Biotek, model: Synergy HT). The OD600 values were recorded at designated time points (every 15 or 30

min or every hour) and the resulting growth curves were analyzed by Microsoft Excel software.

\section{Analysis of binding interaction by isothermal titration calorimetry (ITC)}

Isothermal titration calorimetry (ITC) experiments were performed on a Microcal VPITC microcalorimeter (Northampton, MA), which was calibrated using the built-in electrical calibration check. All ITC experiments were conducted in buffer A (13.5 mM NaCl, $150 \mathrm{mM}$ $\mathrm{KCl}, 20 \mathrm{mM}$ HEPES, $0.22 \mathrm{mM} \mathrm{Na} \mathrm{HPO}_{4}, 0.44 \mathrm{mM} \mathrm{KH}_{2} \mathrm{PO}_{4}, 120 \mu \mathrm{M} \mathrm{MgCl}, 120 \mathrm{nM} \mathrm{CaCl}$, $100 \mu \mathrm{M} \mathrm{MgSO}_{4}$ in $\mathrm{ddH} 2 \mathrm{O}$ at $\mathrm{pH} 7.3$ and $25^{\circ} \mathrm{C}$ ), which was constituted to resemble intracellular conditions. Both the RNAs in the reaction cell and the aminoglycosides (neomycin-B, paromomycin, tobramycin, and kanamycin-A and -B) were prepared in buffer A. All solutions were degassed at room temperature immediately prior to use. Titrant solution $(10 \mu \mathrm{L})$ of each aminoglycoside was added from a $300 \mu \mathrm{L}$ microsyringe at an interval of $150 \mathrm{sec}$ into the stirred sample cell $(1.4288 \mathrm{~mL})$ containing the RNA oligonucleotides at a stirring rate of $310 \mathrm{rpm}$ at $25^{\circ} \mathrm{C}$. To correct for heats of dilution resulting from the ligands, control experiments were performed by making the identical injections of the titrant solution into a cell containing only buffer and then these values were subtracted from the titration of the titrant solution into the reaction cell. Data were analyzed 
using nonlinear least-squares curve fitting in Origin7.0 (Origin Lab Corp., Northampton, MA) using the standard one and two binding site models supplied within Origin7.0.

\section{Determination of IC50 values for aminoglycosides}

We determined the IC50 values for neomycin-B, paromomycin, tobramycin, kanamycin-A and $-B$ by varying the concentrations of the aminoglycosides and determining their growth curves under each condition. The IC50 values were calculated from the OD600 measurements representing the cell densities at stationary phase at $16 \mathrm{~h}$. Table 1 summarizes the calculated IC50s.

\section{Cloning of aptamer sequences}

For cloning the 1 mer and 3mer aptamers, we used double-stranded synthetic DNA oligos obtained from ISU DNA Facility that were inserted in the multiple cloning site and ligated with T4 DNA ligase. The multimers were cut from the pZERO carrier plasmid, inserted in the multiple cloning site and ligated with T4 DNA ligase. All clones were confirmed to be correct by sequencing done at ISU DNA Facility. 


\section{RESULTS AND DISCUSSION}

\section{Decrease in IC50 for several different aminoglycosides was observed in cells expressing aptamers compared with controls}

To test the hypothesis that the expression of aptamers will increase the intracellular concentration of a drug, we used the aminoglycosides and took advantage of the susceptibility of $\mathrm{E}$. coli to inhibition of growth by this drug and used a simple experimental design that monitored the effect of aptamer expression on cell growth (Fig. 1). If the expression of aptamers increases the intracellular concentration of the aminoglycosides as suggested by the mathematical modeling then the IC50 for suppressing bacterial growth by aminoglycosides should be decreased in bacteria that express the aptamers. RNA aptamers that interact with the aminoglycosides (Fig. 2B) were tested. The results showed that the lag phase was extended and the density of cultures at stationary phase was decreased in cells that expressed the aptamers (Fig. 3). Similar results were found with cells that express aptamers previously selected to recognize the aminoglycosides neomycin-B, tobramycin, paromomycin and kanamycins $A$ and $B$. With expressing single unit neomycin aptamer, we were able to decrease the final bacterial densities with lower neomycin-B concentrations, decreasing the IC50 by about 2-fold (Fig. 4). The enhancement in affective concentration of neomycin-B is about 2 to $3 \mu \mathrm{M}$ for the cell densities at $1 \mathrm{~h}$ and 6h time points.

To assess the significance of the finding that expression of aptamers increases the sensitivity of cells to the aptamer targets, we first evaluated the effect of aptamers alone on the bacterial growth. Our results suggest that aptamer expression has no effect on bacterial growth (Figure 5A-F). 


\section{Mathematical prediction I: Increase in drug concentration}

In the mathematical model, as the drug receptor, an aptamer was used and its presence with the appropriate kinetic parameters and its capability to move through the cell cytoplasm was found to be essential to draw its target drug into the cell. As a result, it was predicted that, with the aptamer, a higher intracellular free drug concentration would be achieved than in the absence of the aptamer (Fig. 6, from Levine, H. A.; Boushaba, K.; Nilsen-Hamilton, M. Math Bioscience, 2009, 220, 131). Here we showed that bacteria growth decreases with the aptamer expression as predicted. Also, the binding affinities of RNAs for the aminoglycosides determined by ITC (Table 2) fit within the range of affinities predicted to be effective for drawing in the drug in the mathematical model.

Although the initial results are reported here are consistent with the model, the effect of aptamer expression on the intracellular aminoglycoside concentration has not yet been determined. Measuring the aminoglycoside concentrations is a challenge because they do not have an intrinsic chemical or physical property that we can be utilized for detection. Therefore, the intracellular concentrations of aminoglycosides will be measured commercially available ${ }^{3} \mathrm{H}$-labeled aminoglycosides. These will be used in place of the unlabeled drugs and the radioactive counts from two cultures measured: one with the aptamer expressed and the other with control RNA expressed. This study will provide us with a comparison of the aptamer expressing cells and the cells without aptamers.

\section{Measure extracellular drug concentrations}

An assumption that is made in these studies and in the math modeling is that the extracellular concentration of aminoglycoside remains constant. This will be tested using the 2AP labeled 23mer neomycin RNA aptamer and monitor the increase in its fluorescence upon aminoglycoside binding. An alternate way to determine the extracellular concentration 
of aminoglycosides will be to use the quantitative ninhydrin assay, which is generally sensitive to amino acids, amino sugars and primary, secondary, and tertiary amines and can be used to quantify aminoglycosides that contain both primary and secondary amines. Although the method is quite low in its sensitivity $(\sim 100 \mu \mathrm{M}$ LOD) it will be necessary to partially purify and concentrate the aminoglycoside from the culture medium. This will be done using a cation exchange column.

\section{Mathematical Prediction II: Mobility affects the aptamer action}

In the mathematical model, it was shown that, without diffusion of the aptamer, there was no effect on the inhibitor efficacy. However, when the aptamer was made diffusible, within limits that depend upon the aptamer inhibitor binding affinities $\left(K_{a} s\right)$, the efficacy of the

inhibitor was increased (Fig. 7, from Levine, H. A.; Boushaba, K.; Nilsen-Hamilton, M. Math Bioscience, 2009, 220, 131).

Because larger RNAs are less mobile, we can test the effect of mobility of the aptamer by comparing the effect of a single aptamer in a smaller or larger RNA transcript. If the mathematical prediction is accurate, then we should observe a decrease in aminoglycoside efficacy with aptamers in longer transcripts. Current data in which we have compared transcripts with single aptamers and those with multiple aptamers (larger transcripts) suggest that this prediction is likely to be true because the aptamers are less effective in increasing aminoglycoside efficacy when they are expressed as multimers compared to single unit of neomycin-B aptamer (Fig. 8). However, in these experiments there are two experimental design variables, which are the length of RNA and number of aptamers. 


\section{FIGURE LEGENDS}

Figure 1. DRAGINs bacterial expression system and experimental set-up. The RNA aptamers were cloned downstream of a T7 RNA polymerase promoter with termination signal at the end. Neomycin-B (Neo), kanamycin (Kan), tobramycin (Tob) aptamers, Revresponse element (RRE), thymidylate synthase (TS) mRNA region, and 16S rRNA A site were cloned after the T7 RNA polymerase promoter with a termination signal at the end of the encoded RNA. This expression system depends on the presence of T7 RNA polymerase in the bacterial strain used, BL21(DE3)star.

Figure 2. Chemical structures of neomycin and kanamycin-class aminoglycosides, RNA aptamers used in this study and the working model for the DRAGINs. A) Neomycin-class aminoglycoside antibiotics have a 2-deoxystreptamine (ring II), disubstituted at positions 4 (ring I) and 5 (rings III and IV) while kanamycin-class has substitutions at positions 4 (ring I) and 6 (ring III). B) Predicted secondary structure of the RNA Aptamers. The secondary structures of the aptamers were predicted by RNAstructure4.6 (Matthews et al. 2004). C) Model for the DRAGINs in bacteria.

Figure 3. Comparison of bacterial growth with and without expressed aptamers. These results show that the aptamer expression decreases the growth of bacteria with $7.5 \mu \mathrm{M}$ paromomycin. A) single unit thymidylate synthase mRNA region (closed circle) and B) 3mer 16S A-site minimal unit (closed circle). In both, the control cells with no aptamer is shown with closed squares, C) Two units of RRE (closed circle) and B) single unit thymidylate synthase mRNA region (closed circle) expression decreased the

growth of bacteria with $5 \mathrm{uM}$ kanamycin-B. In both the control cells with no aptamer is shown with closed squares.

Figure 4. Comparison of bacterial growth with single unit of neomycin aptamer. These results indicate that the aptamer decreases the level of bacterial density 
with lower concentration of neomycin-B Final cell densities after normalized to no drug control sample for one unit of neomycin aptamer (closed circle) and control cells with no aptamer at A) $1 \mathrm{~h}$ time point and at B) $6 \mathrm{~h}$ time point with varying concentrations of neomycin-B.

Figure 5. Comparison of bacterial growth with and without the aptamer expression. Our results show that the growth of bacteria is not affected by the expression of aptamers. E. coli BL21 cells with no aptamer containing cells (closed square) are compared with A) kanamycin aptamer B) tobramycin aptamer C) neomycin aptamer D) thymidylate synthase mRNA region E) Rev-response element F) 16S A-site RNA expressing cells. The coefficient of variation is between 5 to $15 \%$ in these experiments, the average of minimum of three samples represented here.

Figure 6. Comparison of the mean total inhibitor concentration with and without the aptamer as a function of time. This figure is taken from Boushaba et al. [4]. Here it is predicted that aptamer can concentrate the drug by more than 10 fold.

Figure 7. Sensitivity of the mean efficiency to the aptamer-inhibitor off rate and the mobility of the aptamer and the target. A) if the aptamer diffuses and the drug target does not diffuse. B) Drug target diffuses at twice the rate of the aptamer. As seen the effect of aptamer is negligible since drug target can move around the cell and can interact with the drug before it is pumped out by the efflux pumps.

Figure 8. Comparison of bacterial growth with single and multiaptamers. These results indicate that the aptamer number of aptamers affect the growth of bacteria differently with 2.5uM kanamycin-B. A) One unit of neomycin aptamer (closed circle) and B) 16 mer of neomycin aptamers (closed circle). In both the control cells with no aptamer is shown with closed squares. 16mer expressing cells rescued the effect of aptamers observed with kanamycin-B. 


\section{FIGURES AND TABLES}

Figure 1

\begin{tabular}{|c|c|}
$\begin{array}{c}\text { T7 RNA } \\
\text { Polymerase } \\
\text { Promoter }\end{array}$ \\
Experimentamers \\
$>$ Grow the bacterial cultures overnight \\
$>$ Dilute them 1 to 100 into new medium with IPTG \\
$>$ Induce them for 1.0 hour with 1.0mM IPTG \\
$>$ Check their growth by measuring OD600
\end{tabular}


Figure 2

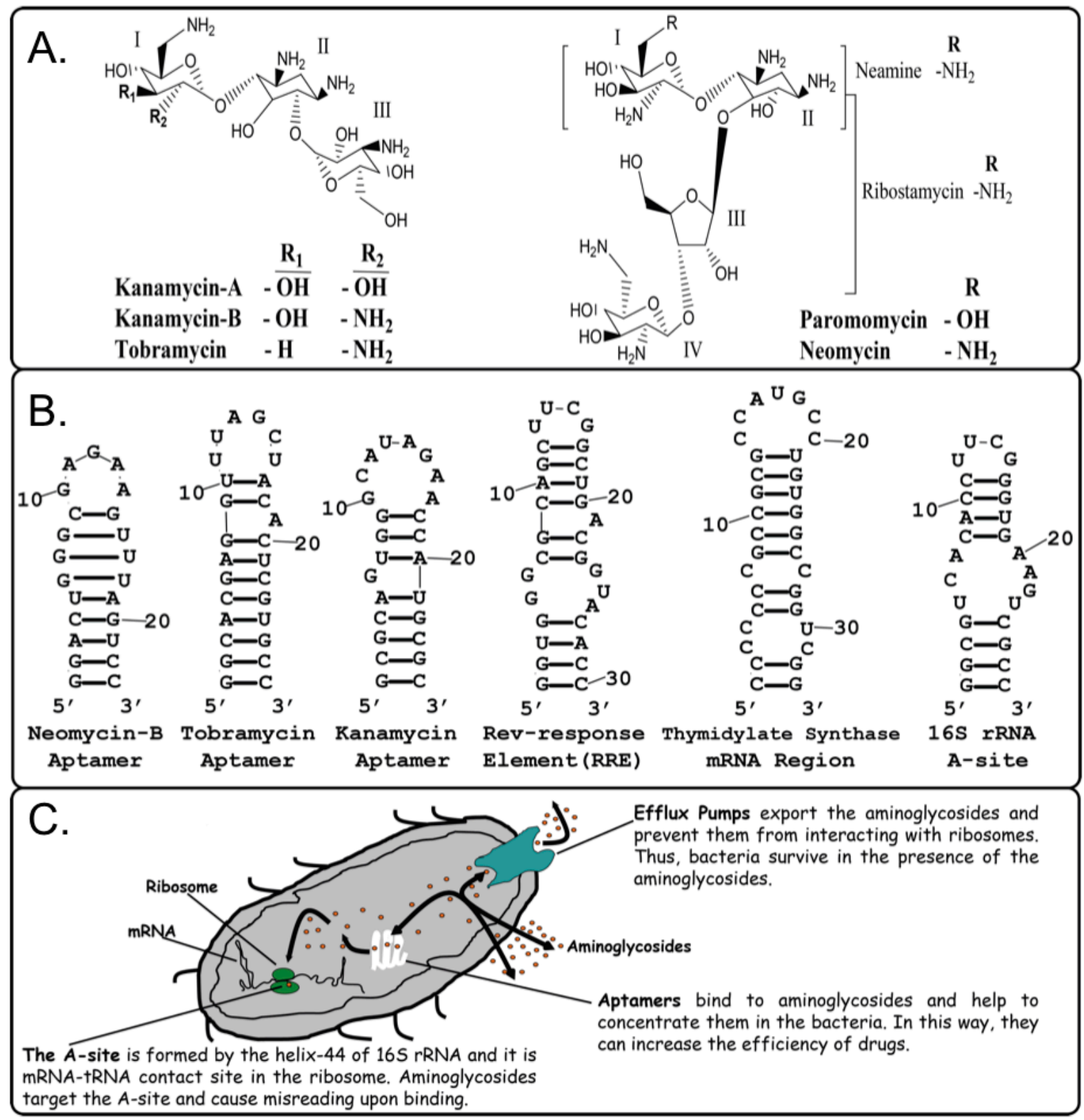


Figure 3
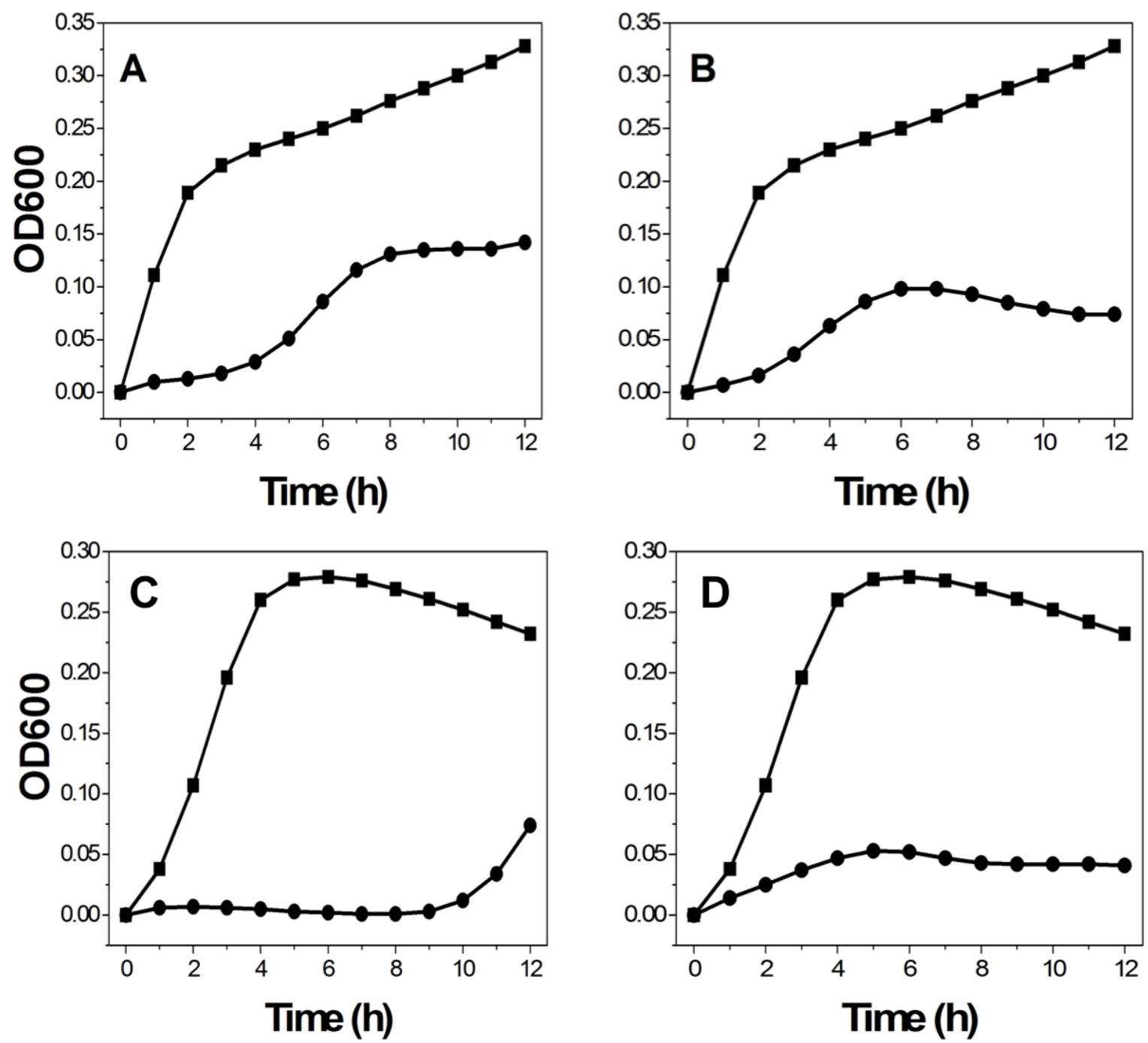
Figure 4
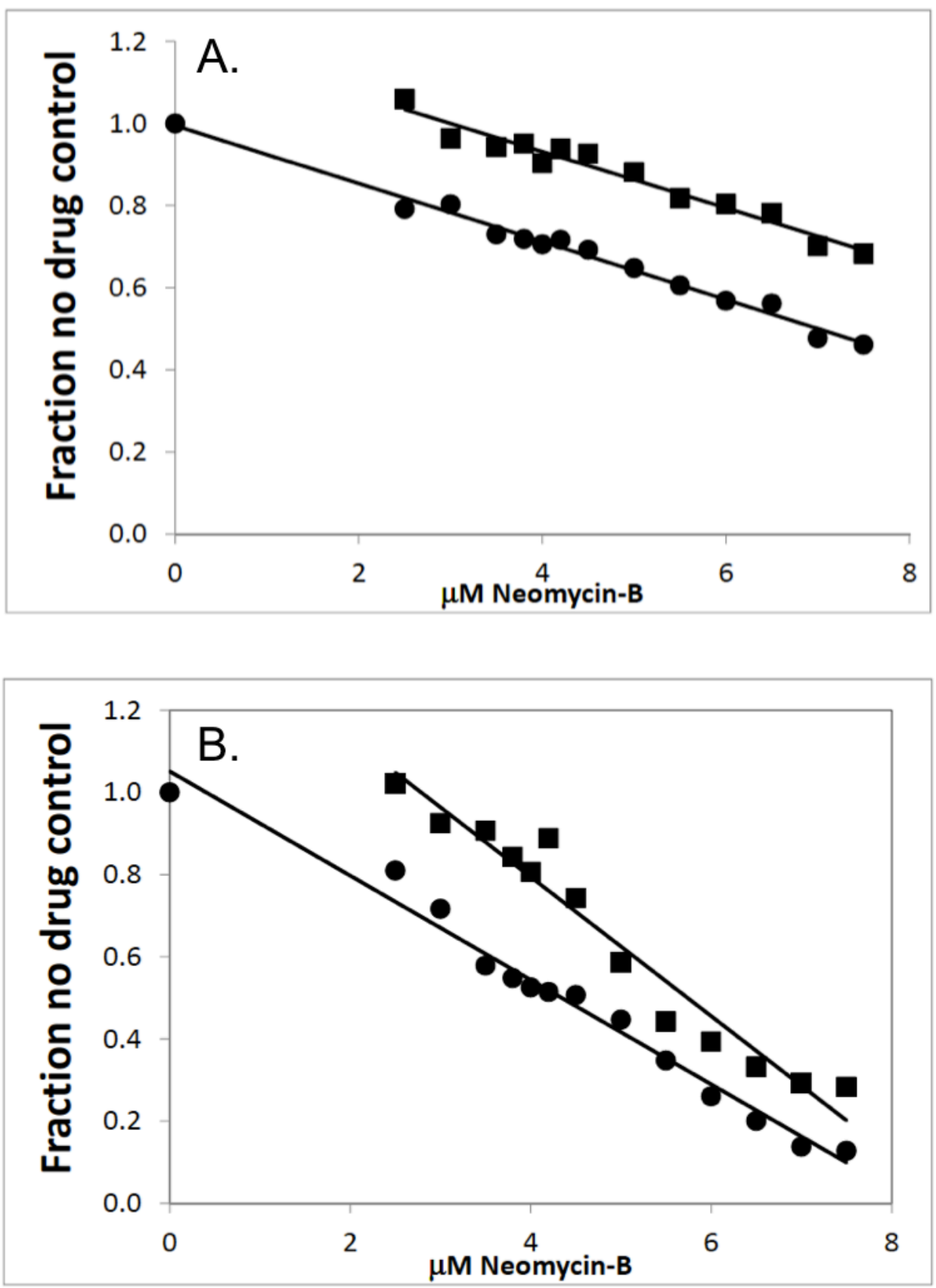
Figure 5
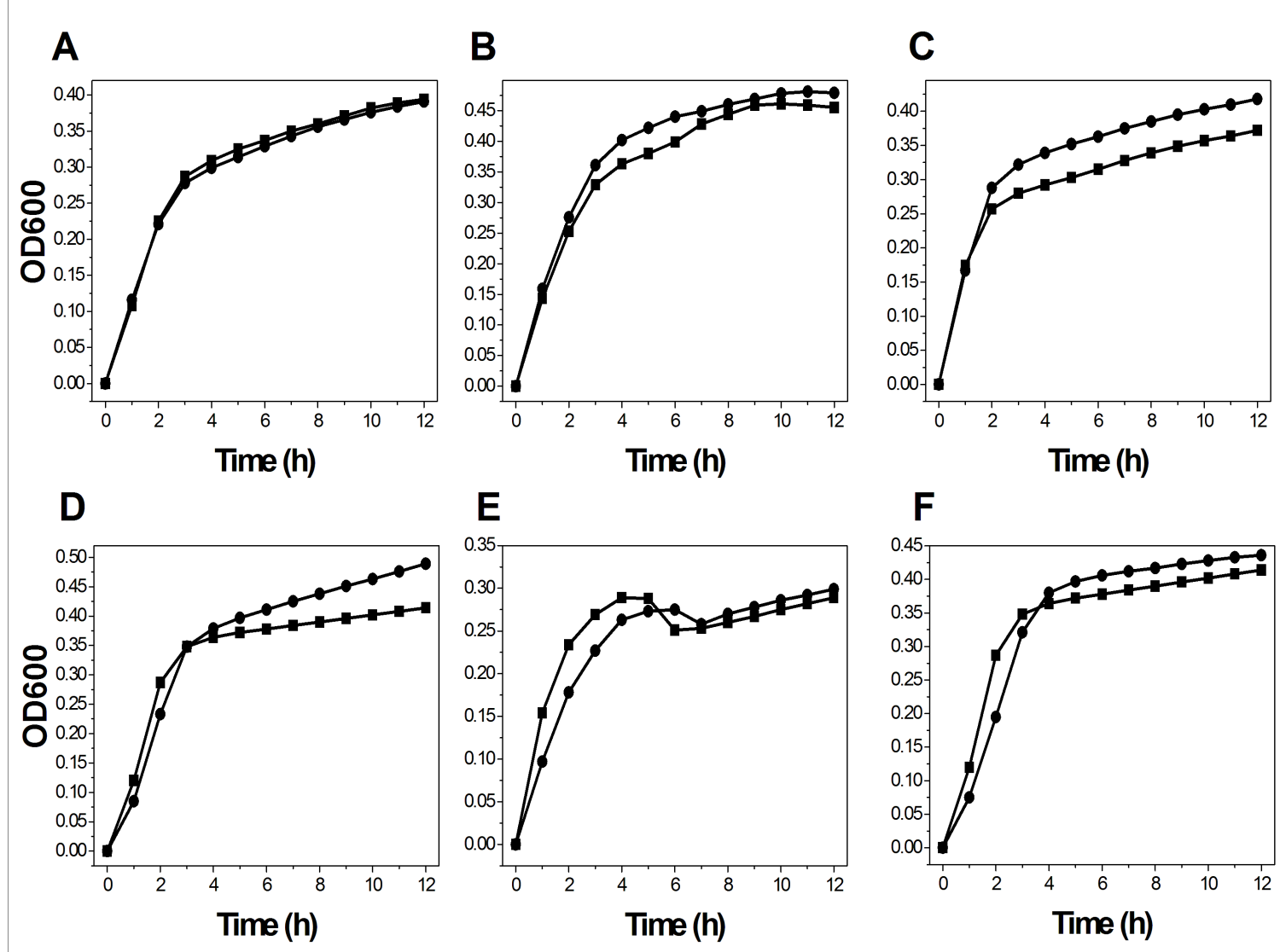
Figure 6

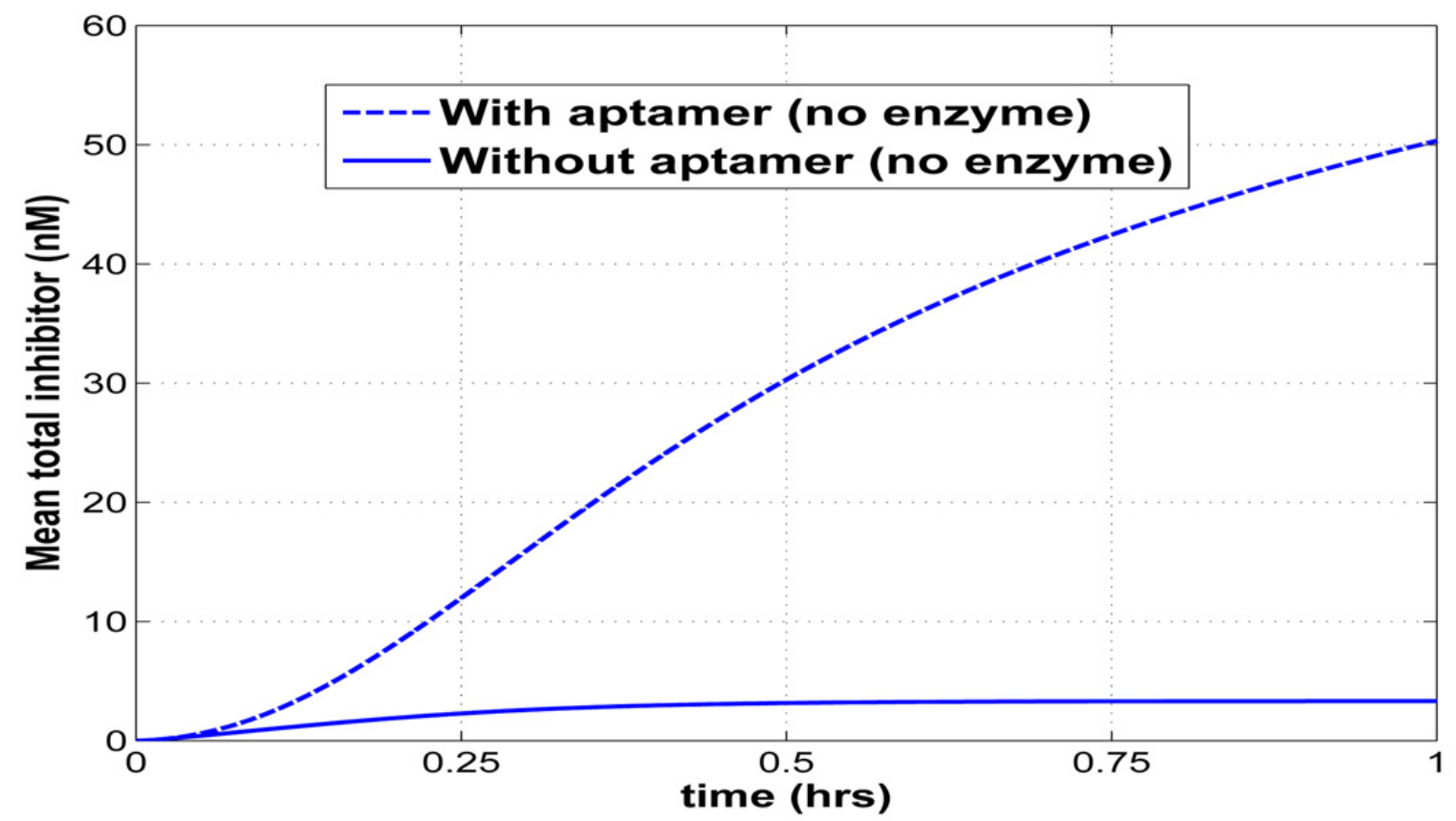


Figure 7

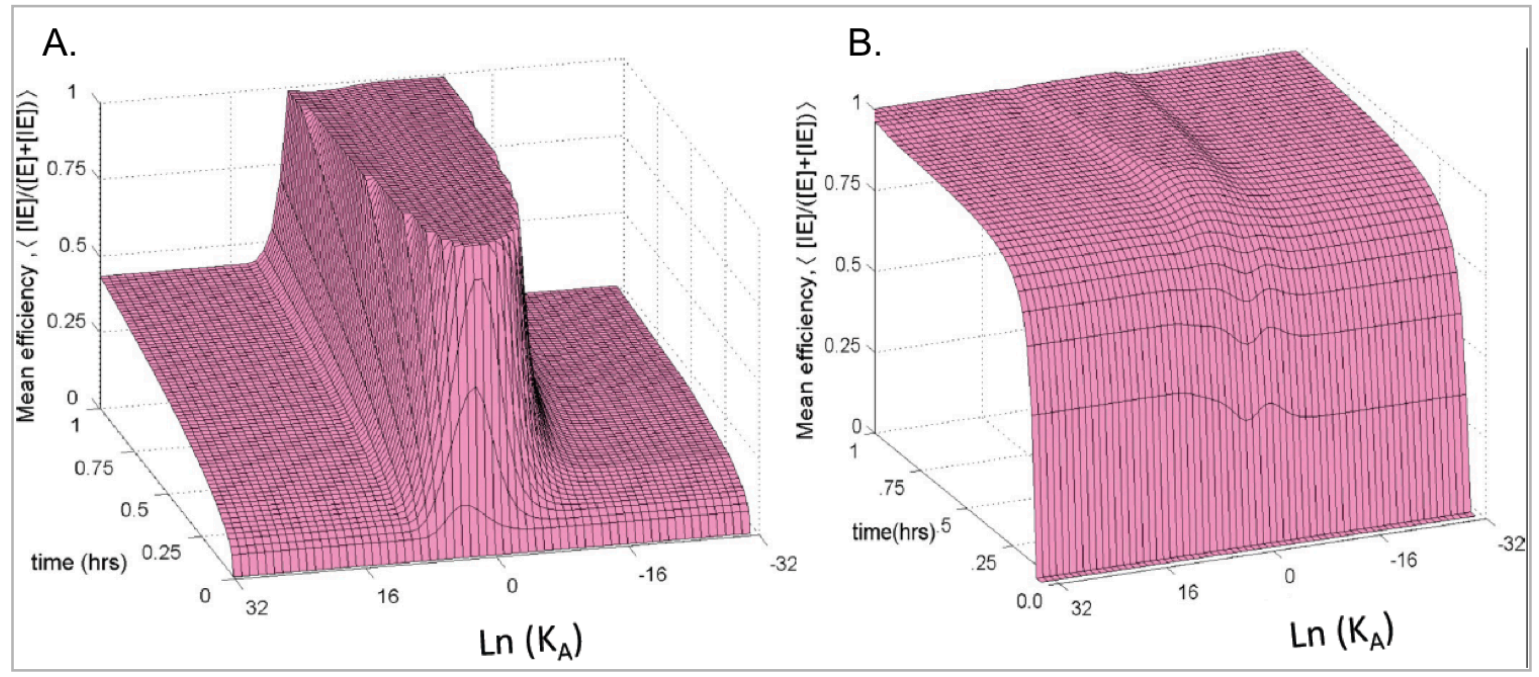

Figure 8
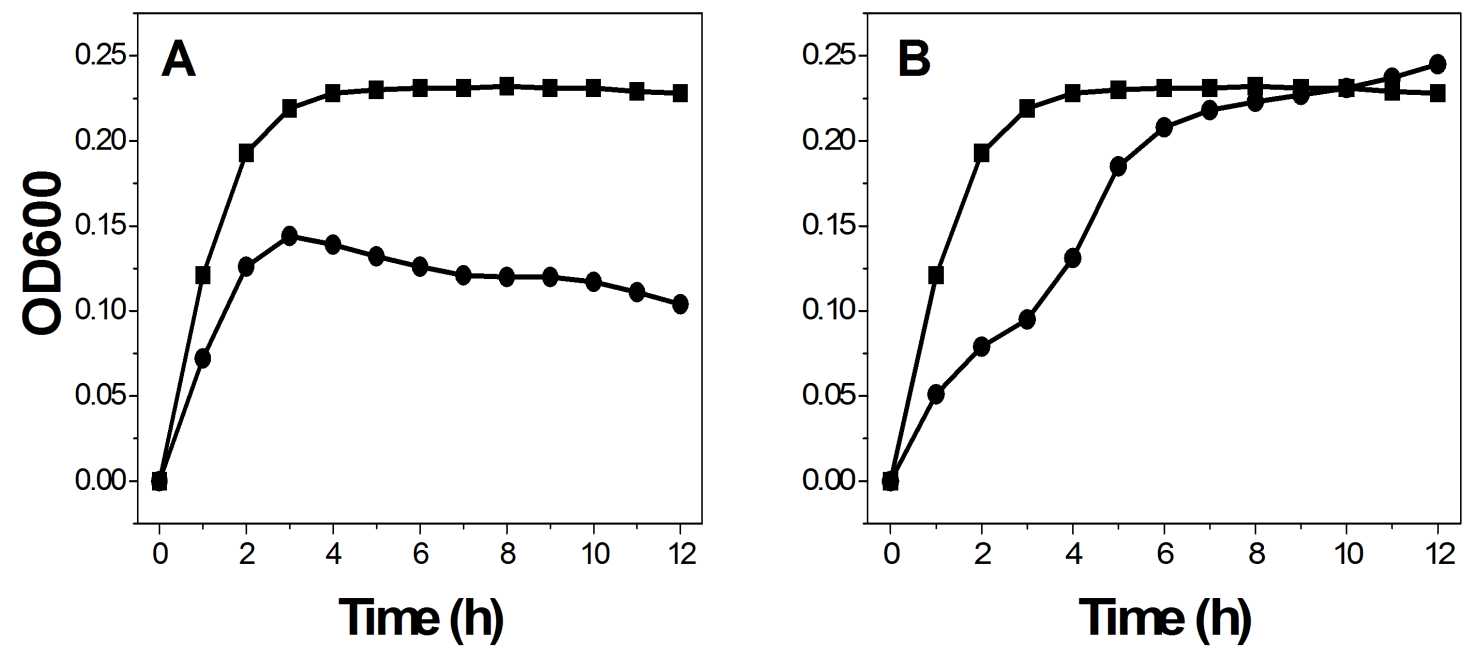
Table 1. IC50 values for neomycin- and kanamycin-class aminoglycosides.

\begin{tabular}{lccc}
\hline & \multicolumn{3}{c}{$\mathrm{IC50}(\mu \mathrm{M})$} \\
\cline { 2 - 4 } Neomycin-B & Lag Phase & Log Phase & Stationary Phase \\
Paromomycin & 1.7 & 4.8 & 6.3 \\
Kanamycin-B & 1.9 & 13 & 17.7 \\
Kanamycin-A & 1.8 & 6.4 & 6.2 \\
Tobramycin & 2.9 & 9.4 & 9.9 \\
\hline
\end{tabular}


Table 2. Dissociation constants of RNAs used in the DRAGINs.

\begin{tabular}{lcccccc}
\hline & \multicolumn{5}{c}{ Dissociation Constants $(\mu \mathrm{M})$} \\
\cline { 2 - 7 } & Neomycin-B & Tobramycin & Kanamycin & Rev- & Thymidylate & 16S rRNA \\
& Aptamer & Aptamer & Aptamer & Response & Synthase & Decoding \\
& & & Element & mRNA region & region \\
Neomycin-B & $0.29 \pm 0.06$ & $2.2 \pm 0.5$ & $6.3 \pm 0.65$ & $3.54 \pm 0.68$ & $0.88 \pm 0.11$ & $1.93 \pm 0.58$ \\
Paromomycin & $1.4 \pm 0.19$ & $17.2 \pm 1.96$ & $9.1 \pm 1.0$ & $27 \pm 4.95$ & $6.29 \pm 3.49$ & $5.31 \pm 1.85$ \\
Kanamycin-B & $0.75 \pm 0.26$ & $2.43 \pm 1.37$ & $5.26 \pm 0.39$ & $8.13 \pm 1.41$ & $6.5 \pm 3.2$ & $8.9 \pm 3.54$ \\
Kanamycin-A & $43 \pm 3.6$ & $73.8 \pm 3.96$ & $14.4 \pm 2.77$ & $32.2 \pm 3.39$ & $10.3 \pm 6.65$ & $37.1 \pm 4.38$ \\
Tobramycin & $4.8 \pm 0.95$ & $0.17 \pm 0.03$ & $7.0 \pm 0.53$ & $4.98 \pm 0.75$ & $5.75 \pm 1.87$ & $10.3 \pm 3.87$ \\
\hline
\end{tabular}




\section{REFERENCES}

1. Chen, G.K., et al., Preferential expression of a mutant allele of the amplified MDR1 (ABCB1) gene in drug-resistant variants of a human sarcoma. Genes Chromosomes Cancer, 2002. 34(4): p. 372-83.

2. Davies, R., et al., Regulation of P-glycoprotein 1 and 2 gene expression and protein activity in two MCF-7/Dox cell line subclones. Br J Cancer, 1996. 73(3): p. 307-15.

3. Nobili, S., et al., Pharmacological strategies for overcoming multidrug resistance. Curr Drug Targets, 2006. 7(7): p. 861-79.

4. Boushaba, K., H. Levine, and M.N. Hamilton, A mathematical feasibility argument for the use of aptamers in chemotherapy and imaging. Math Biosci, 2009. 220(2): p. 131-42.

5. Choi, K.H., Intracellular expression of the T-cell factor-1 RNA aptamer as an intramer. Mol. Cancer Ther., 2006. 5: p. 2428-2434.

6. Burke, D.H. and D.G. Nickens, Expressing RNA aptamers inside cells to reveal proteome and ribonome function. Brief Funct Genomic Proteomic, 2002. 1(2): p. 169-88.

7. Werstuck, G. and M.R. Green, Controlling gene expression in living cells through small molecule-RNA interactions. Science, 1998. 282(5387): p. 296-8.

8. Wright, G.D., A.M. Berghuis, and S. Mobashery, Aminoglycoside antibiotics. Structures, functions, and resistance. Adv Exp Med Biol, 1998. 456: p. 27-69.

9. Moazed, D. and H.F. Noller, Interaction of antibiotics with functional sites in $16 S$ ribosomal RNA. Nature, 1987. 327: p. 389-394.

10. Fourmy, D., et al., Structure of the A site of Escherichia coli $16 S$ ribosomal RNA complexed with an aminoglycoside antibiotic. Science, 1996. 274(5291): p. 1367-71.

11. Fourmy, D., S. Yoshizawa, and J.D. Puglisi, Paromomycin binding induces a local conformational change in the A-site of 16S rRNA. J Mol Biol, 1998. 277: p. 333-345.

12. Woodcock, J., et al., Interaction of antibiotics with $A$ - and P-site-specific bases in $16 S$ ribosomal RNA. Embo J, 1991. 10: p. 3099-3103.

13. Karimi, R. and M. Ehrenberg, Dissociation rate of cognate peptidyl-tRNA from the A-site of hyper-accurate and error-prone ribosomes. Eur J Biochem, 1994. 226: p. 355-360.

14. Xavier, K.A., P.S. Eder, and T. Giordano, RNA as a drug target: methods for biophysical characterization and screening. Trends Biotechnol, 2000. 18(8): p. 349-56.

15. Hermann, T., Strategies for the Design of Drugs Targeting RNA and RNA-Protein Complexes. Angew Chem Int Ed Engl, 2000. 39(11): p. 1890-1904. 
16. Walter, F., Q. Vicens, and E. Westhof, Aminoglycoside-RNA interactions. Curr Opin Chem Biol, 1999. 3(6): p. 694-704.

17. Hermann, T., Rational ligand design for RNA: the role of static structure and conformational flexibility in target recognition. Biochimie, 2002. 84(9): p. 869-75.

18. Chow, C.S. and F.M. Bogdan, A Structural Basis for RNAminus signLigand Interactions. Chem Rev, 1997. 97(5): p. 1489-1514.

19. Zapp, M.L., S. Stern, and M.R. Green, Small molecules that selectively block RNA binding of HIV-1 Rev protein inhibit Rev function and viral production. Cell, 1993. 74(6): p. 969-78.

20. Wang, Y., K. Hamasaki, and R.R. Rando, Specificity of aminoglycoside binding to RNA constructs derived from the 16S rRNA decoding region and the HIV-RRE activator region. Biochemistry, 1997. 36(4): p. 768-79.

21. Cho, J. and R.R. Rando, Specificity in the binding of aminoglycosides to HIV-RRE RNA. Biochemistry, 1999. 38(26): p. 8548-54.

22. Mei, H.Y., et al., Inhibition of an HIV-1 Tat-derived peptide binding to TAR RNA by aminoglycoside antibiotics. Bioorg Med Chem Lett, 1995. 5: p. 2755-2760.

23. Wang, S., et al., Binding of neomycin to the TAR element of HIV-1 RNA induces dissociation of Tat protein by an allosteric mechanism. Biochemistry, 1998. 37(16): p. 5549-57.

24. Stage, T.K., K.J. Hertel, and O.C. Uhlenbeck, Inhibition of the hammerhead ribozyme by neomycin. Rna, 1995. 1(1): p. 95-101.

25. Tor, Y., T. Hermann, and E. Westhof, Deciphering RNA recognition: aminoglycoside binding to the hammerhead ribozyme. Chem Biol, 1998. 5(11): p. R277-83.

26. Earnshaw, D.J. and M.J. Gait, Hairpin ribozyme cleavage catalysed by aminoglycoside antibiotics and the polyamine spermine in the absence of metal ions. Nucleic Acids Res, 1998. 26: p. 5551-5561.

27. Rogers, J., et al., Inhibition of the self-cleavage reaction of the human hepatitis delta virus ribozyme by antibiotics. J Mol Biol, 1996. 259: p. 916-925.

28. Chia, J.S., et al., Inhibition of hepatitis delta virus genomic ribozyme self-cleavage by aminoglycosides. J Biomed Sci, 1997. 4: p. 208-216.

29. Kirk, S.R. and Y. Tor, tRNA(Phe) binds aminoglycoside antibiotics. Bioorg Med Chem, 1999. 7(9): p. 1979-91. 
30. Walter, F., et al., Binding of tobramycin leads to conformational changes in yeast $\operatorname{tRNA}(A s p)$ and inhibition of aminoacylation. Embo J, 2002. 21(4): p. 760-8.

31. Lee, J., et al., An approach to enhance specificity against RNA targets using heteroconjugates of aminoglycosides and chloramphenicol (or linezolid). J Am Chem Soc, 2004. 126(7): p. 1956-7.

32. Means, J.A. and J.V. Hines, Fluorescence resonance energy transfer studies of aminoglycoside binding to a T box antiterminator RNA. Bioorg Med Chem Lett, 2005. 15(8): p. 2169-72.

33. Tok, J.B., J. Cho, and R.R. Rando, Aminoglycoside antibiotics are able to specifically bind the 5'-untranslated region of thymidylate synthase messenger RNA. Biochemistry, 1999. 38(1): p. 199-206.

34. Corvaisier, S., V. Bordeau, and B. Felden, Inhibition of transfer messenger RNA aminoacylation and trans-translation by aminoglycoside antibiotics. J Biol Chem, 2003. 278(17): p. 14788-97.

35. Mikkelsen, N.E., et al., Inhibition of RNase P RNA cleavage by aminoglycosides. Proc Natl Acad Sci USA, 1999. 96: p. 6155-6160.

36. von Ahsen, U., J. Davies, and R. Schroeder, Antibiotic inhibition of group I ribozyme function. Nature, 1991. 353: p. 368-370.

37. von Ahsen, U., J. Davies, and R. Schroeder, Non-competitive inhibition of group I intron RNA self-splicing by aminoglycoside antibiotics. J Mol Biol, 1992. 226: p. 935-941.

38. Waldsich, C., K. Semrad, and R. Schroeder, Neomycin B inhibits splicing of the td intron indirectly by interfering with translation and enhances missplicing in vivo. Rna, 1998. 4: p. 1653-1663.

39. Varani, L., et al., Structural basis for recognition of the RNA major groove in the tau exon 10 splicing regulatory element by aminoglycoside antibiotics. Nucleic Acids Res, 2000. 28(3): p. 710-9.

40. Wallis, M.G., et al., A novel RNA motif for neomycin recognition. Chem Biol, 1995. 2: p. 543-552.

41. Jiang, L., et al., Saccharide-RNA recognition in a complex formed between neomycin $B$ and an RNA aptamer. Structure, 1999. 7(7): p. 817-27.

42. Jiang, L. and D.J. Patel, Solution structure of the tobramycin-RNA aptamer complex. Nature Struct Biol, 1998. 5: p. 769-774. 
43. Kwon, M., et al., In vitro selection of RNA against kanamycin B. Mol Cells, 2001. 11(3): p. 303-11.

44. Wang, $\mathrm{H}$. and $\mathrm{Y}$. Tor, Electrostatic interactions in RNA-aminoglycosides binding. J Am Chem Soc, 1997. 119: p. 8734-8735. 


\section{CHAPTER 5. GENERAL CONCLUSIONS}

\section{THE SPECIFICITY OF AN RNA APTAMER IS HIGHLY DEPENDENT ON THE IONIC ENVIRONMENT}

Aptamers are nucleic acid molecules specifically selected for a target of interest, which can be a small molecule or a whole cell. In general, the family of aptamers obtained through SELEX specifically bind to that target with very strong affinity in the range of pM to mM. However, our results have shown that specificity of the RNA aptamer selected for neomycin-B is highly dependent on the buffer components used in binding assays. The first report for the selection of the 23mer neomycin-B RNA aptamer showed that, in a $\mathrm{pH} 7.4$ buffer containing potassium cacodylate, magnesium chloride and ammonium chloride, the aptamer binds to neomycin-B with $>100$-fold better affinity than a very closely related analog, paromomycin [1]. By contrast, in our binding assays, we used a $\mathrm{pH} 7.3$ buffer containing HEPES, $\mathrm{NaCl}, \mathrm{KCl}, \mathrm{MgCl}_{2}$ and $\mathrm{CaCl}_{2}$ and found that the aptamer has only a 5 -fold better binding affinity to neomycin-B than paromomycin, which was similarly observed in phosphate-saline buffer. This indicated that the binding ability of the aptamer changes with its surroundings. We further characterized the binding of ten aminoglycosides to the aptamer under identical buffer conditions (HEPES, $\mathrm{NaCl}, \mathrm{KCl}, \mathrm{MgCl}_{2}$ and $\mathrm{CaCl}_{2}, \mathrm{pH} 7.3$ ) by ITC. These results showed that both neomycin- and kanamycin-family aminoglycosides bound the aptamer. In parallel studies, the order of binding was captured with very high accuracy by molecular docking analysis with the single exception of ribostamycin. These initial binding studies identified the importance of the first two rings for RNA recognition that make specific contacts within the binding pocket of the neomycin-B RNA aptamer. Because the RNA was considered rigid in the docking analysis, we performed molecular dynamics (MD) simulations, which have been recently used to study the dynamics of nucleic acids and 
aptamer-target interactions. The proper use of MD simulations yields results in good agreement with experimental data and our aim with MD simulation was to extend structural and dynamical information beyond the limitations of current experimental techniques. In MD simulations, we observed that the $16^{\text {th }}$ adenine residue was highly flexible in the absence of the ligand, which is probably the transition from bound to unbound state. This simulation enabled us to provide a simulated 3D-structure of unoccupied neomycin-B RNA aptamer with experimental validations. By replacing A16 with 2-aminopurine (2AP) and detecting the increase in its fluorescence, we confirmed the change in A16 position upon ligand binding. This result indicated that $\mathrm{A} 16$ was changing its position from a stacked position, where its fluorescence is quenched to a more solvent accessible position, where it can emit more of its fluorescence [2]. However, the difference in the level of increase in 2AP fluorescence with neomycin-B, ribostamycin and paromomycin is suggest that interactions with these ligands or differential effects of the ligands on aptamer structure also contribute to the fluorescence change.

In conclusion, we have characterized the binding of ten aminoglycosides to the 23mer neomycin-B RNA aptamer by ITC and molecular docking. These studies were complemented with MD simulations and 2AP fluorescence studies with further analyses to show that the ionic conditions were important for both aminoglycoside binding and selectivity of the aptamer. In this study, we have combined computational and experimental approaches to understand the details of aminoglycoside RNA interactions. Our results show that the specificity and affinity of this RNA for its ligands differ with the ionic environment and suggest that other aptamers should be carefully examined for similar effects of their environment. 


\section{APTAMER-TARGET INTERACTIONS CAN BE UTILIZED FOR IN VIVO APPLICATIONS}

Although aptamers are selected in vitro, they can be integrated into expression platforms for intracellular use. In our study, we have shown that aptamers can be successfully utilized for in vivo applications. As recently shown by Paige et al., RNA aptamers once expressed inside the cell can be utilized to detect cellular metabolites including S-adenosyl methionine and ADP [3], in our studies we utilized RNA aptamers in IMAGEtags to detect gene expression in real time and in DRAGINs to increase the number of drug molecules inside cells to enhance drug efficacy. Thus, our results have shown proof of principle in yeast and bacterial cells for two new methods that utilize intracellular transcribed aptamers that recognize the aminoglycosides, neomycin-B, tobramycin and PDC. The next phase of the IMAGEtags project will be to develop the capability of detecting gene expression in mammalian cells. For DRAGINs, the next phase will be to determine if the same principle of increasing intracellular drug levels 2 to 5 -fold holds in mammalian cells.

\section{MATHEMATICAL MODELING OF APTAMER EXPRESSION MAY CHALLENGE US TO IMPROVE THE DRAGINS SYSTEM}

In 2009, our research group in collaboration with mathematicians in the Department of Mathematics at lowa State University mathematically predicted the utility of aptamers to concentrate drugs inside mammalian cells [4]. With DRAGINs, we have demonstrated the utilization of aptamers inside bacterial cells to increase drug concentrations. Our data showed that the aptamer expression system works as predicted by the previous mathematical model. We believe that further mathematical modeling of DRAGINs system may challenge us to answer other critical basic questions and may help us improve our system. We are collaborating with Drs. Howard Levine and YeonJung Seo to better understand the DRAGINs system by mathematical modeling, this time with a compartment 
model for prokaryotes. Once completed, the predictions of the model will be tested by wetlab experiments.

\section{UNDERSTANDING RNA-AMINOGLYCOSIDE INTERACTIONS MAY HELP TO DEVELOP BETTER ANTIBIOTICS}

Aminoglycosides have been on the market for the last several decades. However, the emergence of resistant bacterial strains may eventually make these antibiotics useless. Understanding the interaction of aminoglycosides with their target RNAs can help resolve this problem. We have characterized the neomycin-B RNA aptamer aminoglycoside interactions; however, there are other natural and synthetic RNAs that can interact with the aminoglycosides. These nonspecific interactions may increase the chance of side effects through aminoglycoside use. Pinpointing the details of aminoglycoside-RNA interactions, might lead to the development of more potent aminoglycoside-based antibiotics. The results of these studies may allow researchers to design more specific drugs to the bacterial target sites with less nonspecific binding. Using structural information in designing new drugs may significantly decrease the amount of trial-error period spent in the lab for a new compound that may take years to appear in the market. Indeed, 3D structures of some of these RNAs in complex with aminoglycosides are already available for use in molecular docking and MD simulations to design more potent antibiotics. However, structural data for some of these RNAs are not available and to fill this gap, recent 3D prediction programs like MC-Sym [5], might help to get necessary data and push development of the RNA and pharmaceutical fields one step further.

\section{RNA-AMINOGLYCOSIDE INTERACTIONS MAY HELP US DEVELOP RIBOSWITCHES}

Natural riboswitches generally bind to small metabolites and control gene expression. They play critical roles as check points in gene expression pathways and limit the amount of 
ATP spent that would be otherwise wasted on unnecessary translation. In addition to the natural riboswitches, recently, artificial riboswitches have been designed to control gene expression by external stimuli [6]. In this study, several neomycin-B riboswitches were identified for designing specific gene expression platforms that are regulated by neomycin-B. These systems can be improved with a better understanding of RNA-aminoglycoside interactions. Experimental and computational approaches can also be applied here to develop more responsive or better riboswitches.

\section{FUTURE PERSPECTIVE}

It has been more than two decades since two groups $[7,8]$ invented the aptamer selection procedure independently. Many studies have demonstrated aptamer applications in medicine and Macugen, approved by FDA (Food and Drug Administration) by the end of 2004, is the first aptamer drug. It is a polyethyleneglycol (PEG)-modified anti-VEGF aptamer that is used to treat age-related macular degeneration. In addition to their potential applications in medicine, aptamers also serve as good biosensors in vitro. Recently, aptamer selection has been done through robotics and with the addition of high-throughput sequencing, it is now less time consuming to obtain an aptamer than previously. This high throughput and highly efficient selection technology should soon meet the high demand of aptamers that recognize various biologically significant targets both in vitro and in vivo. Once available in sufficient numbers, aptamers have the potential to replace most of the antibodies used in diagnosis.

As aptamers have been selected in vitro, they have also been identified in nature. The first natural riboswitch to be reported regulates genes involved in riboflavin synthesis in bacteria [9]. Following these studies, many riboswitches that contain aptamer domains have been identified by Breaker's lab at Yale [10]. Riboswitches are RNA elements in upstream 
regions of the coding mRNA that can specifically bind to metabolites with their aptamer domains and regulate gene expression both at the transcriptional and translational level. Perhaps, the identified riboswitches may be just small portion of the natural aptamers identified so far and genomes may be filled with aptamers. I think bioinformatics will play an essential role to identify the remaining aptamers hidden in the genomes.

Also, aptamers selected for aminoglycoside antibiotics have particular importance because these antibiotics have been used to fight against pathogenic Gram-negative bacteria. A better understanding of aptamer aminoglycoside interactions, as has been developed in this study, can be utilized to design more effective aminoglycoside-based drugs, which may help to kill the newly arising antibiotic resistance bacterial strains.

In addition to their applications to drug development, aminoglycoside-RNA interactions can be used as in vivo sensors in the form of riboswitches, in which aminoglycosides serve as external stimuli. Therefore, a better understanding of RNAaminoglycoside interactions may also help researchers to in vitro select new riboswitches and/or aptamers, characterize them and apply these into different in vivo platforms. One of the fields utilizing these platforms is "synthetic biology" for which designed expression systems or short metabolic circuits are frequently utilized. Computational approaches may help to develop better riboswitches for these circuits. Such improved RNA-aminoglycoside pairs might be applied to controlling different pathways used by synthetic biologists in their designed expression systems. 


\section{REFERENCES}

1. Wallis, M.G., et al., A novel RNA motif for neomycin recognition. Chem Biol, 1995. 2: p. 543-552.

2. Ballin, J.D., et al., Local RNA conformational dynamics revealed by 2-aminopurine solvent accessibility. Biochemistry, 2008. 47(27): p. 7043-52.

3. Paige, J.S., et al., Fluorescence imaging of cellular metabolites with RNA. Science, 2012. 335(6073): p. 1194.

4. Boushaba, K., H. Levine, and M.N. Hamilton, A mathematical feasibility argument for the use of aptamers in chemotherapy and imaging. Math Biosci, 2009. 220(2): p. 131-42.

5. Parisien, M. and F. Major, The MC-Fold and MC-Sym pipeline infers RNA structure from sequence data. Nature, 2008. 452(7183): p. 51-5.

6. Weigand, J.E., et al., Screening for engineered neomycin riboswitches that control translation initiation. RNA, 2008. 14(1): p. 89-97.

7. Ellington, A.D. and J.W. Szostak, In vitro selection of RNA molecules that bind specific ligands. Nature, 1990. 346(6287): p. 818-22.

8. Tuerk, C. and L. Gold, Systematic evolution of ligands by exponential enrichment: RNA ligands to bacteriophage T4 DNA-polymerase. Science, 1990. 249: p. 505-510.

9. Gelfand, M.S., et al., A conserved RNA structure element involved in the regulation of bacterial riboflavin synthesis genes. Trends Genet, 1999. 15(11): p. 439-42.

10. Winkler, W., A. Nahvi, and R.R. Breaker, Thiamine derivatives bind messenger RNAs directly to regulate bacterial gene expression. Nature, 2002. 419(6910): p. 952-6. 


\section{APPENDIX I. CONTRIBUTION TO THE DEVELOPMENT OF IMAGETAGS (INTRACELLULAR MULTIAPTAMER GENETIC TAGS) FOR DETECTING PROMOTER ACTIVITY IN LIVING CELLS}

In our research group, we have developed a novel way for imaging promoter activity in real time in vivo. In this method, we have utilized strings of RNA aptamers as in vivo RNA tags called IMAGEtags (Intracellular MultiAptamer GEnetic tags). This system can be expressed from a promoter of choice. The tobramycin, neomycin-B and PDC RNA aptamers have been utilized for this system and expressed in yeast from the GAL1 promoter. I have used the neomycin-B RNA aptamers, for which paromomcyin is a ligand, in this project to construct neomycin-B IMAGEtags. For imaging, we used yeast cells that expressed the neomycin-B IMAGEtags or a control RNA to test our system. These cells were incubated with a mixture of two paromomycin derivatives conjugated with the FRET pairs, Cy3 and Cy5, respectively. With these constructs, the transcribed aptamers can be imaged after activation of the promoter by galactose. This work has been submitted to Nature Methods.

In this project, I also cloned kanamycin aptamers as IMAGEtags in a carrier plasmid called pZERO (as shown in Table 1); but, the binding affinity of kanamycin-B to its cognate aptamer was not high enough for in vivo studies. However, if we can find another Cy3 and Cy5-labeled ligand that can bind to the kanamycin aptamer with better affinity, we can also utilize these constructs as IMAGEtags.

In this project, I utilized neomycin-B aptamer; however, I had to use paromomycinCy3 and paromomycin-Cy5 as the FRET pairs to detect the promoter activity in living yeast cells. This was due to chemical limitations in labeling neomycin-B at a single position, with the consistent result being two products of the conjugation reaction between neomycin-B 
and either Cy3 or Cy5-succinimidyl ester (data not shown). Because it has only single active primary amino group, paromomycin could be labeled with Cy3 or Cy5 at a single position. So, I tested if the neomycin-B RNA aptamer could bind to paromomycin and determined the dissociation constant as $\sim 1.5 \mu \mathrm{M}$. This was a good enough affinity to test the neomycin-B IMAGEtags in cell studies. The binding affinities were determined via ITC using buffer A (chapter 2), which is designed to have similar concentrations of free monoand divalent cations as the cell cytoplasm. I calculated the dissociation constants for Cy3paromomycin as $\sim 4 \mu \mathrm{M}$, shown in figure 2 and for paromomycin-Cy5 as $\sim$ about $3 \mu \mathrm{M}$, shown in figure 3.

\section{FRET Image Acquisition}

I detected the initial FRET with paromomycin-Cy3 and paromomycin-Cy5 and observed that the 24mer neomycin-B IMAGEtags gave a higher FRET signal than the control cells (0mer) shown in figure 1. The cells were observed using an inverted Nikon Eclipse 200 fluorescence microscope with a $63 \mathrm{X}$ objective and immersion oil. Cells were excited by a $568 \mathrm{~nm}$ Argon/Krypton laser to measure sensitized emission using emission filters for FRET of $700-750 \mathrm{~nm}$. However, this microscope had the limitation that it could not also be used to obtain bright field images, which would have allowed the identification of all yeast cells in each field. To obtain bright field images I then used the Leica SP5X laser scanning confocal microscope with a $63 \mathrm{X}$ objective and immersion oil. This microscope has the capability to obtain the bright field images as well as individual fluorescence signals either from Cy3 or Cy5. Cells were excited by a $550 \mathrm{~nm}$ white light laser (WLL) and FRET images were taken to measure sensitized emission using emission filters for FRET of 660$710 \mathrm{~nm}$. 


\section{Test to check the stability of IMAGEtags}

It is well known that $E$. coli tend to eliminate repeated sequences from its genome or from plasmids that they carry [1-3]. To determine the stability of the IMAGEtags, I transformed E. coli with plasmids containing IMAGEtags, and picked up three colonies from each transformation. Then, I performed two tests on the plasmids isolated from each colony; agarose gel electrophoresis and with sequencing. In both of the assays, the results showed that the number of repeated aptamers stayed the same in both kanamycin and neomycin-B IMAGEtags with a single exception being a 15 mer obtained from originally 16 mer neomycinB IMAGEtag (Table 2) 
TABLES AND FIGURES

Table 1. Results from the cloned inserts with multiaptamers.

\begin{tabular}{|lll|}
\hline Repeated Sequence & $\begin{array}{l}\text { Source and number } \\
\text { of initial repeats }\end{array}$ & $\begin{array}{l}\text { Number of } \\
\text { repeats after one } \\
\text { round }\end{array}$ \\
\hline $\begin{array}{l}\text { Neomycin-B aptamer } \\
\text { (GGACUGGGCGAGAAGUUUAGUCC) }\end{array}$ & Oligonucleotides (6) & $6,8^{*}$ \\
\cline { 2 - 3 } & Vector (8) & $16,23^{*}, 24,32$ \\
\hline Kanamycin aptamer & & 7 \\
\hline (GCGCAGUGGGCAUAGAACCAUGCGC) & Oligonucleotides $(7)$ & $79^{*}, 12^{*}, 14,26,28$, \\
\cline { 2 - 3 } & & 56 \\
\hline
\end{tabular}

Cloning of neomycin-B and kanamycin aptamers was performed to obtain multiaptamers that were used in IMAGEtags. The initial sequence repeats were inserted as annealed oligonucleotides or were in a vector from a previous round. We obtained 8, 16, 23, and 24 repeats and $9,12,14,26,28,56$ units from the neomycin-B and kanamycin aptamer clonings, respectively. Each aptamer repeat was separated by a four adenine nucleotides used as a linker and the asterisk indicates that the number of repeats obtained was not expected from the components used during cloning. 
Table 2. Stability of cloned repeated sequences.

\begin{tabular}{|c|c|c|c|c|}
\hline \multirow[t]{2}{*}{ Repeated sequence } & \multicolumn{2}{|c|}{ Number of repeats } & \multirow[t]{2}{*}{ Vector } & \multirow[t]{2}{*}{ Analysis Type } \\
\hline & Original & Selected colonies & & \\
\hline \multirow[t]{5}{*}{ Neomycin-B aptamer } & 8 & $8(3)$ & pTrc & Sequencing \\
\hline & 16 & $15(1) ; 16(2)$ & pTrc & Sequencing \\
\hline & 24 & $24(3)$ & pTrc & Sequencing \\
\hline & 24 & $24(4)$ & $p Z X-2$ & Gel electrophoresis \\
\hline & 32 & $32(3)$ & pTrc & Sequencing \\
\hline \multirow[t]{5}{*}{ Kanamycin aptamer } & 3 & $3(3)$ & pTrc & Sequencing \\
\hline & 7 & $7(3)$ & pTrc & Sequencing \\
\hline & 12 & $12(3)$ & pTrc & Sequencing \\
\hline & 14 & $14(3)$ & pTrc & Sequencing \\
\hline & 28 & $28(3)$ & pTrc & Sequencing \\
\hline
\end{tabular}

Plasmids containing a variety of repeated sequences were tested for their stability in E. coli. They were isolated from randomly selected clones of transformed bacteria and tested for the number of sequence repeats by either sequencing or restriction digestion followed by gel electrophoresis of the released insert to determine its size. 


\section{FIGURE LEGENDS}

Figure 1. Comparison of FRET images from 24mer IMAGEtags and Omer control samples. The yeast cells with 24 mer or with empty plasmid were grown overnight. Then, 10uL of the cells were taken from each culture and spun down. The medium was changed to induction medium containing $5 \mu \mathrm{M}$ of Cy3- and Cy5-paromomycin and the cells were incubated for $15 \mathrm{~min}$ at $30^{\circ} \mathrm{C}$. After this, the cells were spun down and washed 3 times with TBS to remove the excess dyes. Finally, the cells were re-suspended in TBS and placed in the $3.5 \mathrm{~cm}$ glass-bottom dishes and the images were taken with either $63 \mathrm{X}$-oil objective (for all 24mer images) or $63 \mathrm{X}$-oil (bottom middle images) and 20X air objectives for the 0mer control samples. A $550 \mathrm{~nm}$ white light laser (WLL) was used to excite the Cy3-paromoycin and FRET images were taken to measure the sensitized emission using emission filters for Cy5 of 660-710 nm.

Figure 2. ITC thermogram from the titration of the neomycin-B RNA aptamer into Cy3paromomycin. The thermodynamic parameters are shown in the squared box and the calculated dissociation constant $\left(\mathrm{K}_{\mathrm{d}}\right)$ was about $4 \mu \mathrm{M}$.

Figure 3. ITC thermogram from the titration of the neomycin-B RNA aptamer in Cy5paromomycin. The thermodynamic parameters are shown in the square box and the calculated dissociation constant $\left(\mathrm{K}_{\mathrm{d}}\right)$ was about $3 \mu \mathrm{M}$. 


\section{FIGURES}

Figure 1
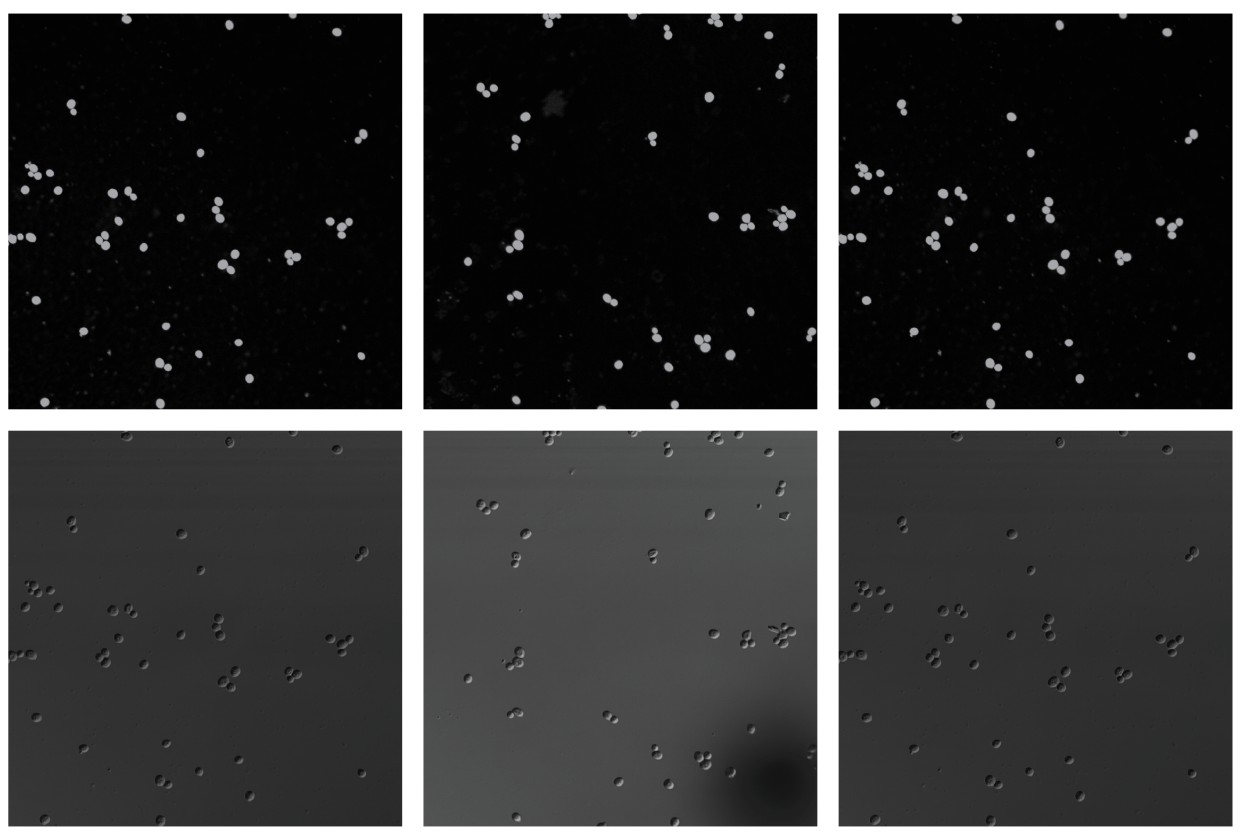

24mer Neomycin-B IMAGEtags
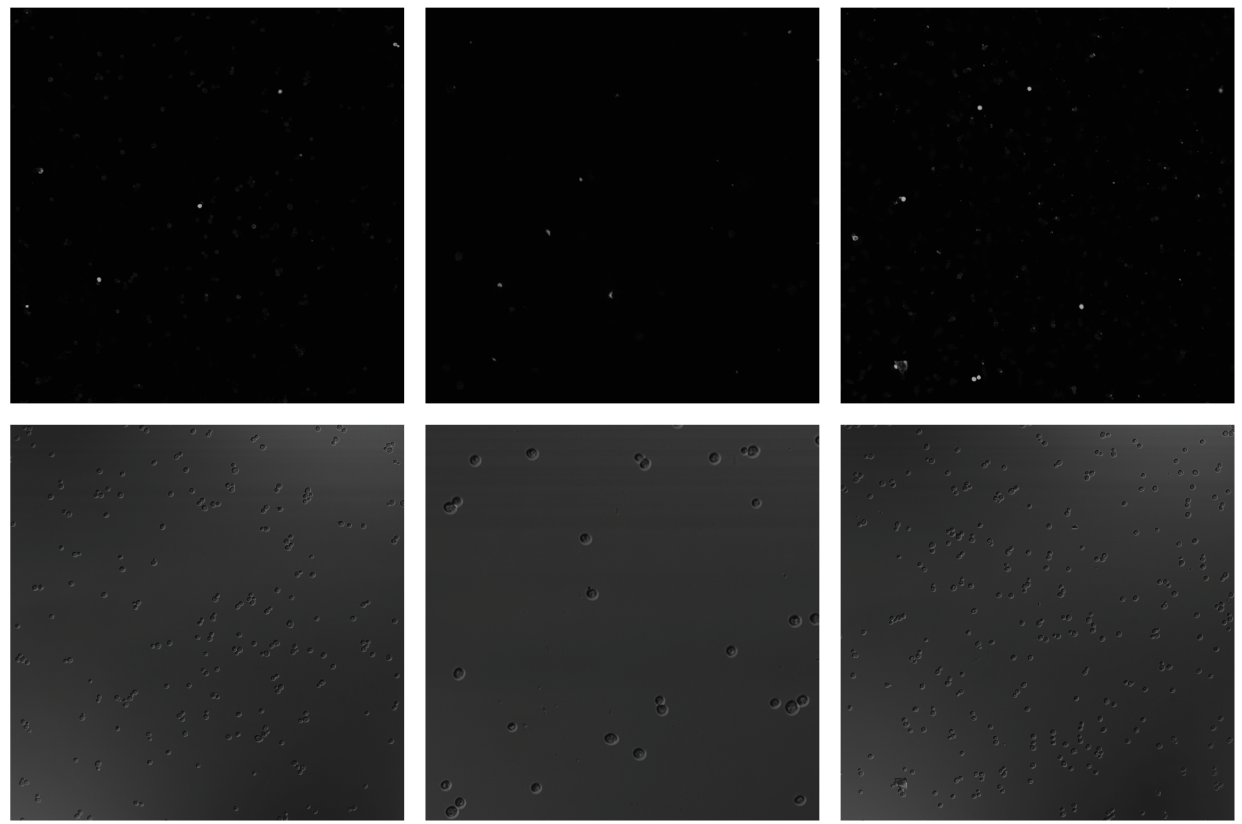

Omer Control samples 
Figure 2

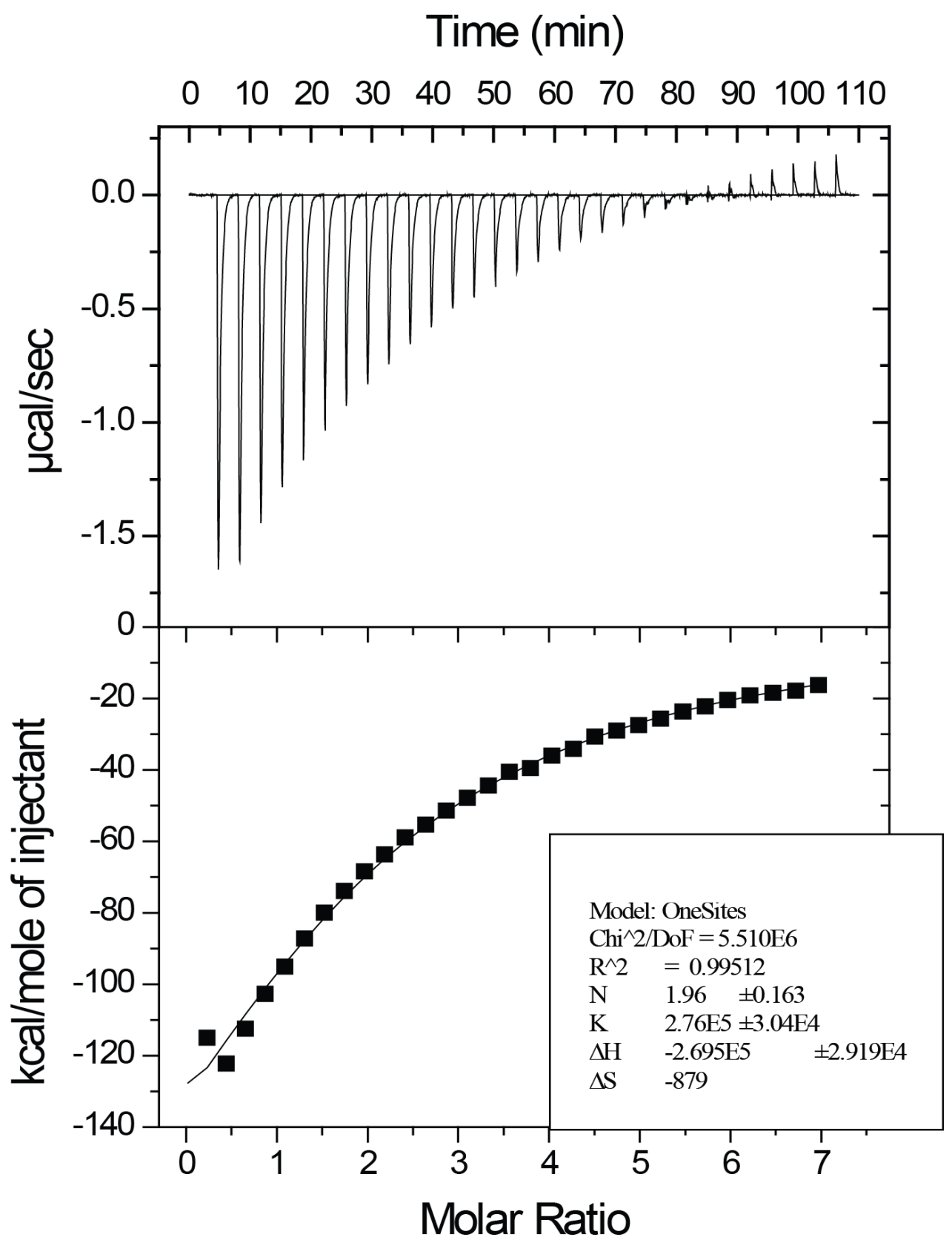


Figure 3

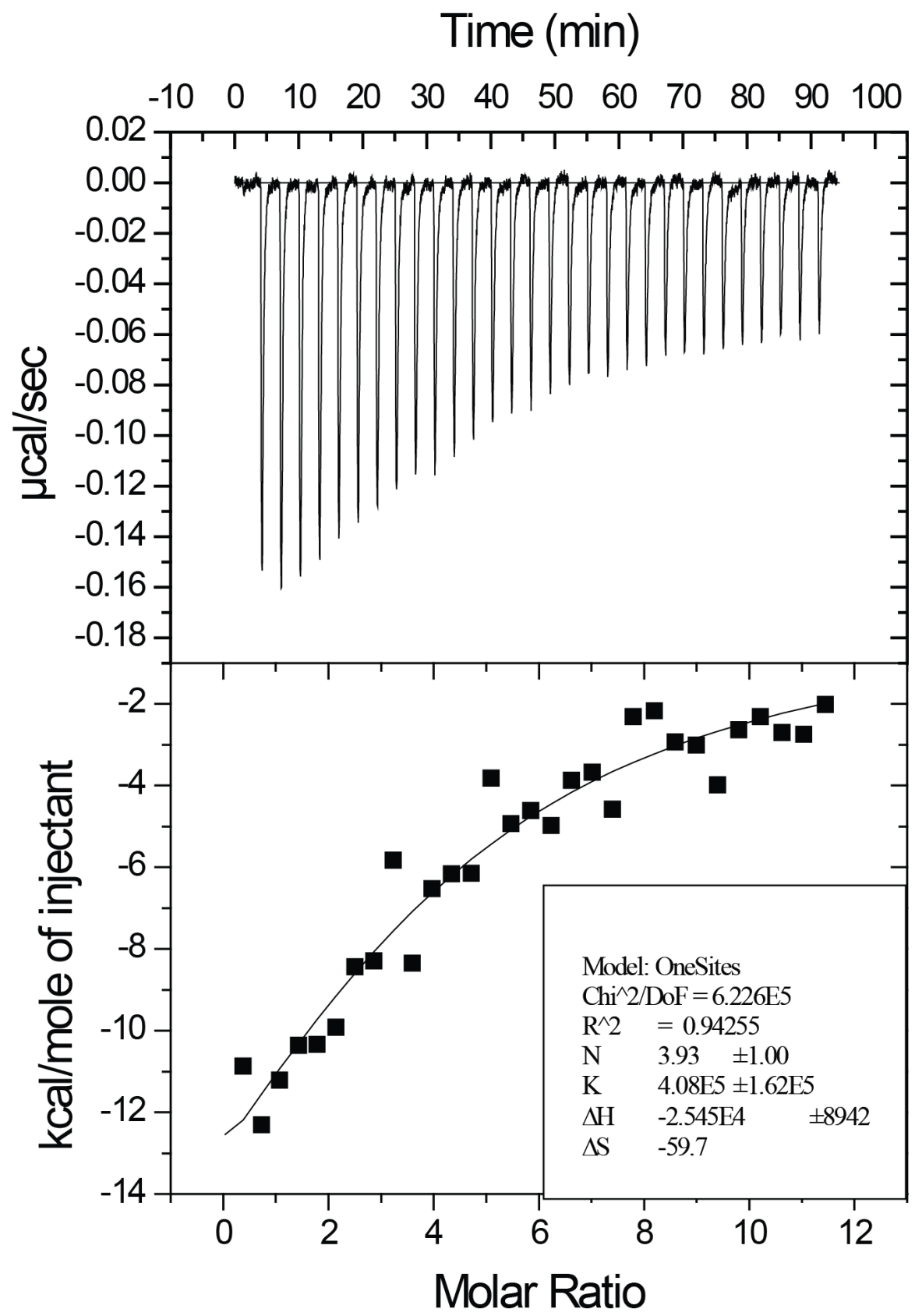




\section{REFERENCES}

1. $\mathrm{Bi}, \mathrm{X}$. and L.F. Liu, A replicational model for DNA recombination between direct repeats. J Mol Biol, 1996. 256(5): p. 849-58.

2. $\mathrm{Bi}, \mathrm{X}$. and L.F. Liu, recA-independent and recA-dependent intramolecular plasmid recombination. Differential homology requirement and distance effect. J Mol Biol, 1994. 235(2): p. 414-23.

3. Rosche, W.A., T.Q. Trinh, and R.R. Sinden, Differential DNA secondary structuremediated deletion mutation in the leading and lagging strands. J Bacteriol, 1995. 177(15): p. 4385-91. 


\section{APPENDIX II. NUCLEAR MAGNETIC RESONANCE (NMR) STUDY OF THE NEOMYCIN-B RNA APTAMER TO DETERMINE THE} CONFORMATION OF A16 UPON AMINOGLYCOSIDE BINDING

As I have detailed in the study of the neomycin-B RNA aptamer in chapter 3 , the $16^{\text {th }}$ adenine (A16) residue was observed as a flexible residue in the MD simulations and results from a study of the 2AP-modified aptamer suggest that this base changes its position from a stacked to a less constrained conformation upon aminoglycoside binding. The fluorescence emission of the 2AP16 is expected to increase upon its release from the stacked position in the loop. To experimentally determine the positioning of A16 in the bound and unbound conformations, we have performed ${ }^{1} \mathrm{H}$, NOESY and TOCSY NMR experiments. The $1 \mathrm{D}$ and 2D assignments for the RNA residues are still in progress and this work will be completed in collaboration with the NMR facility manager, Bruce Fulton at lowa State University.

NMR Protocol: Bruker Avance 700 spectrometer equipped with a $5 \mathrm{~mm} \mathrm{HCN}-\mathrm{Z}$ gradient cryoprobe was used to collect the ${ }^{1} \mathrm{H}$ NMR spectra of all samples. Spectra were acquired at sample temperatures of $25^{\circ} \mathrm{C}$ and $5^{\circ} \mathrm{C}$. All $1 \mathrm{D}{ }^{1} \mathrm{H}$ NMR spectra were acquired using a WATERGATE pulse sequence with water flipback (Bruker sequence p3919fpgp) to minimize solvent saturation transfer. The 1D spectra are the averages of 64 transients. The spectral width was $18182 \mathrm{~Hz}$ (25 ppm). The TOCSY spectra were acquired using MLEV17 isotropic mixing and WATERGATE solvent suppression (Bruker sequence mlevgpph19). NOESY spectra were acquired with WATERGATE and water flipback solvent suppression (Bruker sequence noesyfpgpphrs19). Mixing times of $75 \mathrm{~ms}$ and $200 \mathrm{~ms}$ were used for TOCSY and NOESY spectra, respectively. The samples were prepared as $500 \mu \mathrm{M}$ neomycin-B RNA aptamer alone for unbound and with $500 \mu \mathrm{M}$ neomycin-B for the bound conformations in $10 \mathrm{mM} \mathrm{Na}_{2} \mathrm{HPO} 4$ at $\mathrm{pH} 7.3,95 \% \mathrm{H}_{2} \mathrm{O} / 5 \% \mathrm{D}_{2} \mathrm{O}$. 


\section{FIGURE LEGENDS}

Figure 1. Representative NMR spectra of neomycin-B RNA aptamer samples. Each panel shows a region of the $1 \mathrm{D}{ }^{1} \mathrm{H}$ spectrum and the corresponding region of the $200 \mathrm{~ms}$ NOESY spectrum. All spectra were recorded in $95 \% \mathrm{H}_{2} \mathrm{O} / 5 \% \mathrm{D}_{2} \mathrm{O}$ buffer at $25^{\circ} \mathrm{C}$. A) Base-pair hydrogen bond region of neomycin-B RNA aptamer alone, B) base to 1' region of neomycin-B RNA aptamer alone, $\mathrm{C}$ ) base-pair hydrogen bond region of ligand-bound neomycin-B RNA aptamer, D) base to 1' region of ligand-bound neomycin-B RNA aptamer. 
Figure 1

A
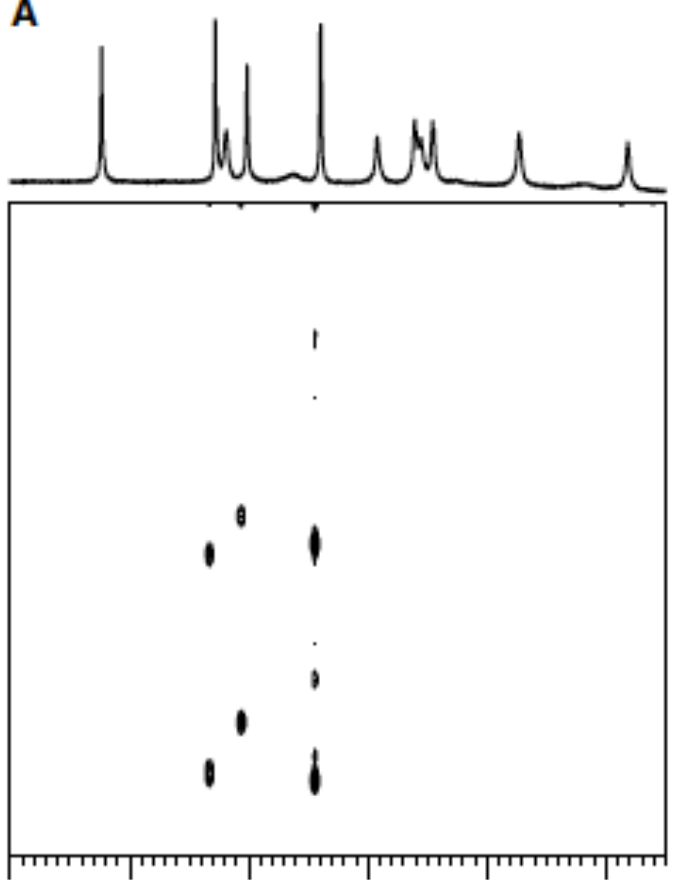

$\begin{array}{lllll}14 & 13 & 12 & 11 & \text { ppm }\end{array}$

C
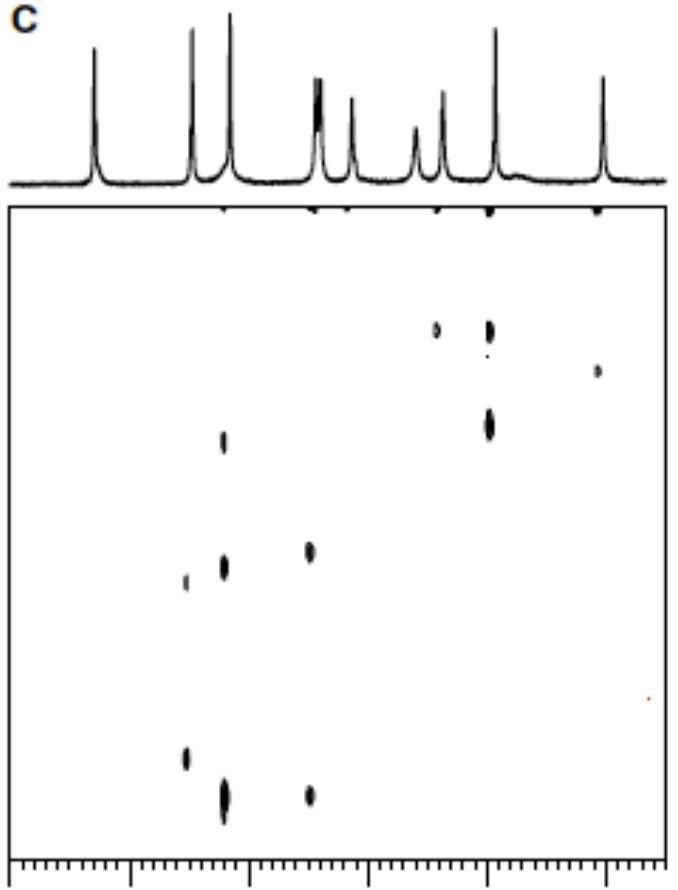

$\begin{array}{lllll}14 & 13 & 12 & 11 & \mathrm{ppm}\end{array}$
B
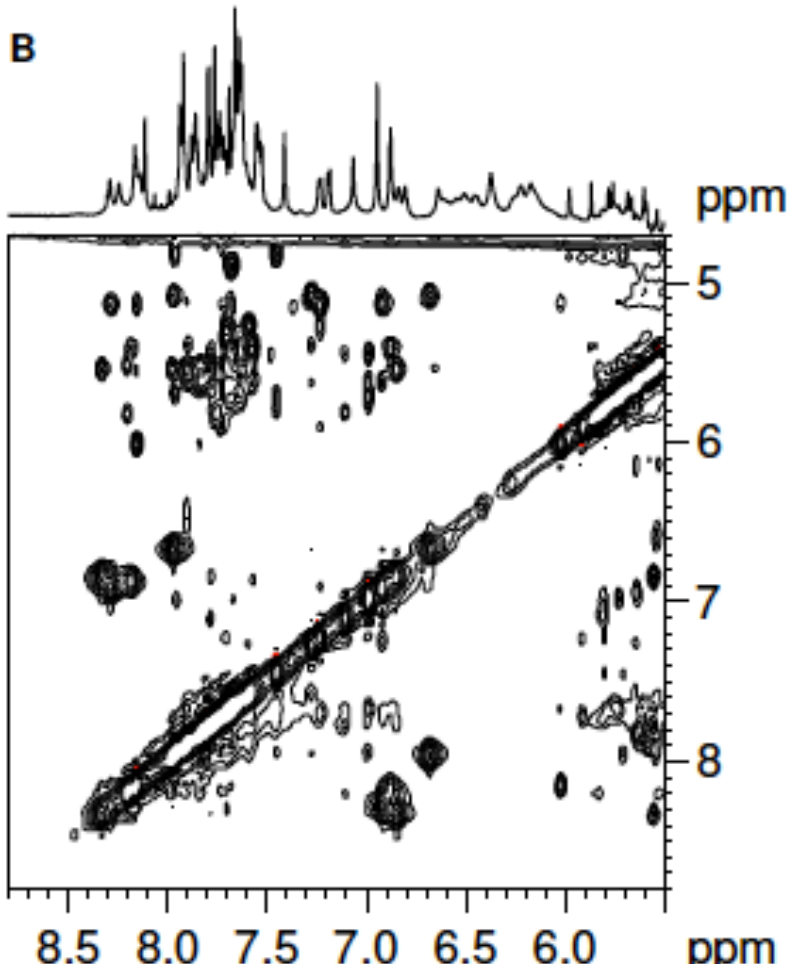

D

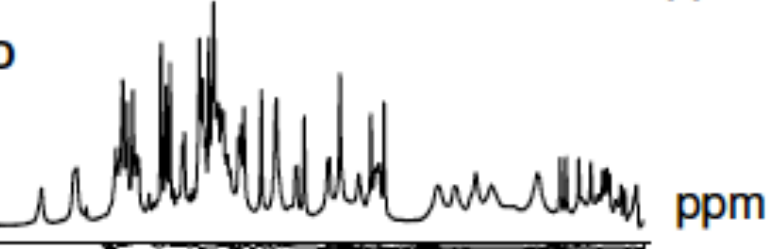

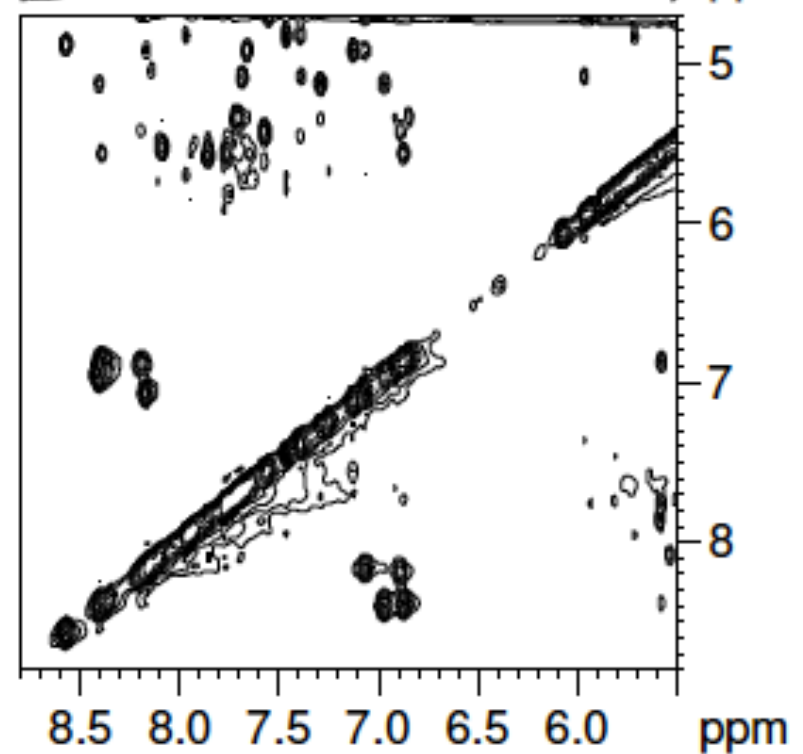




\section{APPENDIX III. EQUILIBRIUM BINDING ANALYSIS OF RIBOSWITCHES THAT BIND TO NEOMYCIN-B}

One of the few-engineered riboswitches for neomycin-B was obtained by an in vivo screening method developed in yeast [1]. In this study, several riboswitches were identified by which neomycin-B can regulate the expression of green fluorescent protein (GFP). The binding affinities for for neomycin-B of these riboswitches were reported to be in the low nM. However, based on my previous observations that aminoglycoside-binding RNAs have the capability to interact with more than one type of aminoglycoside, I decided to test the binding of these neomycin-B riboswitches with ten aminoglycosides by ITC. The riboswitches with the best regulatory capability were chosen for study. My binding studies indicated that these riboswitches behaved very similar to the other aptamers that I tested in that they bind to both neomycin and kanamycin-class aminoglycosides. However, their selectivities were quite different from each other and in some cases neomycin-B was not the highest affinity ligand. For example, 30NEO4A binds to paromomycin better than it binds to neomycin-B. This RNA is one of the best paromomycin binders amongst all the RNAs tested.

Figure 1 shows the predicted secondary structures of the riboswitches examined in this study. These riboswitches share the structural features of having stem I, internal bulge, stem II and loop. These structural features are also observed in most other RNAs that interact with aminoglycosides. Aminoglycosides in general bind to the major-grooves of RNA duplexes and as the internal bulge has a widened major groove, the internal bulge is the most probable binding site in these riboswitches. Figure 2 shows the mechanism of riboswitch action with the presence of neomycin-B inhibiting the initiation of translation. Figure 3 shows the association constants for these three riboswitches with ten different aminoglycosides. The buffer used in these ITC assays was buffer A (chapter 2), which is 
designed to have concentrations of mono- and divalent cations as the cell cytoplasm. These studies were done in preparation for a comparison of riboswitch function in yeast cells

\section{FIGURE LEGENDS}

Figure 1. Predicted secondary structures of the neomycin-B riboswitches. The secondary structures were predicted by using the RNAStructure5.2 software. The free energies were also obtained using the same program and are represented for all RNAs. The nomenclature for the RNAs is as follows: 25,31 and 30 are the lengths, NEO is the abbreviation for neomycin- $\mathrm{B}$ as the original ligand and $2 \mathrm{~A}, 3 \mathrm{~A}, 4 \mathrm{~A}$ identify individual aptamers (or riboswitches).

Figure 2. Schematic representation of aptamer-mediated inhibition of translational initiation. The aptamer is placed upstream of the start codon of GFP. Left: without neomycin the aptamer does not interfere with ribosomal scanning and GFP can be expressed. Right: when neomycin binds to the aptamer, this complex inhibits the small ribosomal subunit and leads to decreased gene expression.

Figure 3. Association constants $\left(K_{a} s\right)$ of the neomycin-B riboswitches for ten different aminoglycosides. The affinities were determined by ITC in buffer $\mathrm{A}(13.5 \mathrm{mM} \mathrm{NaCl}, 150 \mathrm{mM}$ $\mathrm{KCl}, 20 \mathrm{mM}$ HEPES, $0.22 \mathrm{mM} \mathrm{Na}_{2} \mathrm{HPO}_{4}, 0.44 \mathrm{mM} \mathrm{KH}_{2} \mathrm{PO}_{4}, 120 \mu \mathrm{M} \mathrm{MgCl}, 120 \mathrm{nM} \mathrm{CaCl}$, $100 \mu \mathrm{M} \mathrm{MgSO}_{4}$ in $\mathrm{ddH}_{2} \mathrm{O}$ at $\mathrm{pH} 7.3$ at $25^{\circ} \mathrm{C}$ ) and the calculated $\mathrm{K}_{d} \mathrm{~S}$ (dissociation constant = $1 / \mathrm{K}_{\mathrm{a}}$ ) range from $\sim 30 \mathrm{nM}$ to $100 \mu \mathrm{M}$ for these aminoglycoside ligands. 


\section{FIGURES}

Figure 1

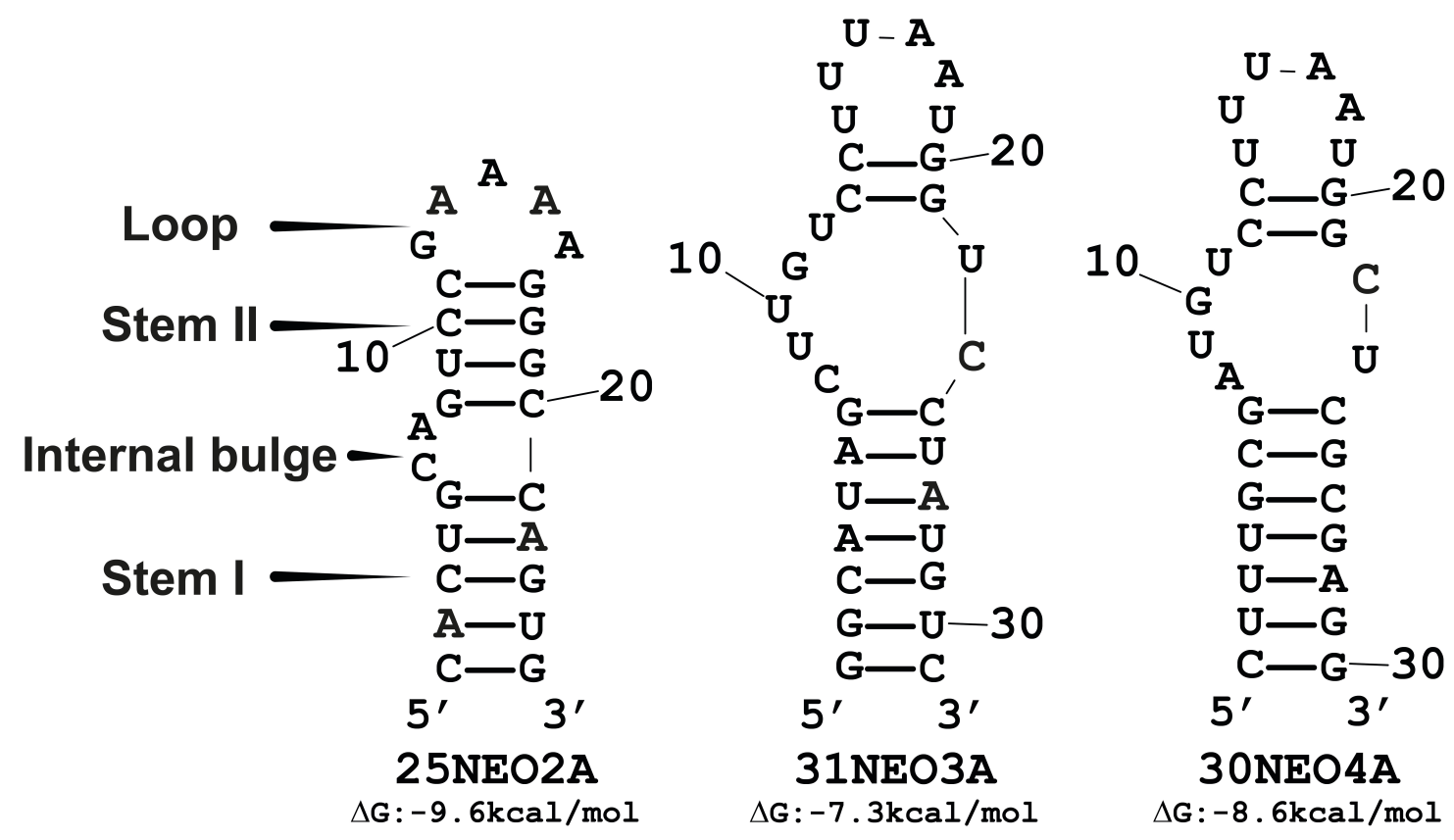

Figure 2
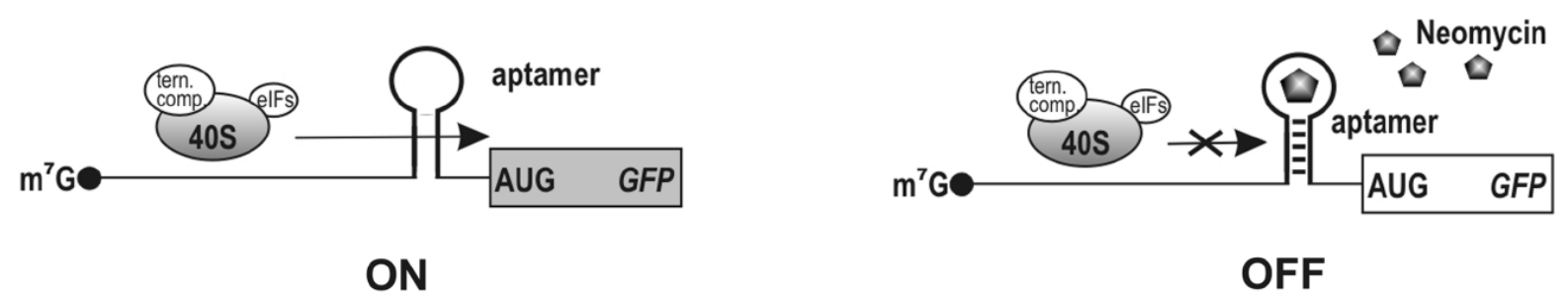
Figure 3

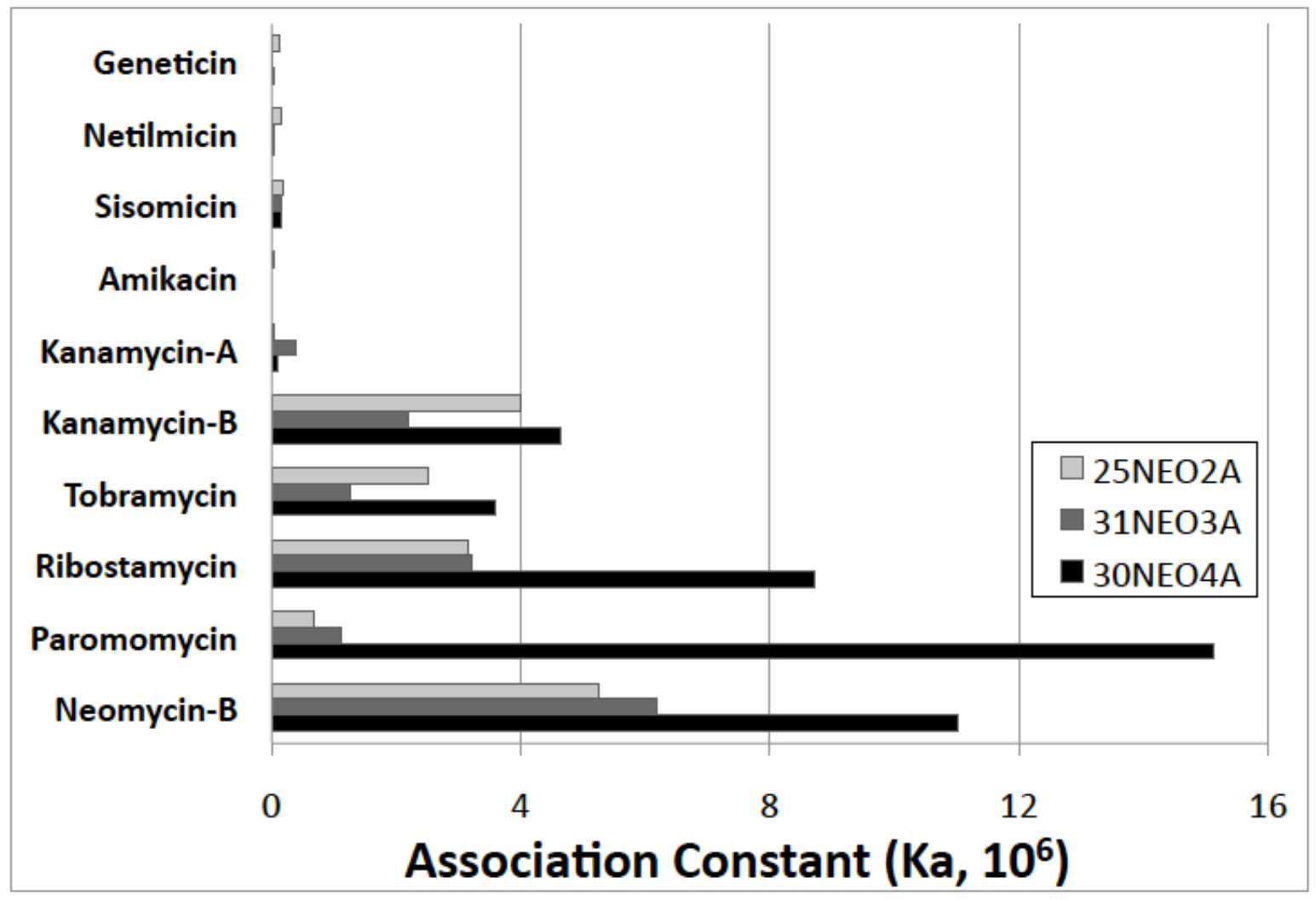

\section{REFERENCE}

1. Weigand, J.E., et al., Screening for engineered neomycin riboswitches that control translation initiation. RNA, 2008. 14(1): p. 89-97. 


\section{ACKNOWLEDGEMENTS}

First, I would like to thank my major professor Dr. Marit Nilsen-Hamilton for her guidance and constant support during my doctoral studies. Without her help, this dissertation would not have been put together and I am deeply grateful for the long discussions and regular meetings that helped me grow as an independent scientist. I could never make it to this point without her dedicated support. Special thanks are given to our lab manager Lee Bendickson who has provided technical supports and helped me with my frontsheets and labnotes. Also, I would like to thank my committee members, Dr. Allen Miller, Dr. Gustavo Maclntosh, Dr. Thomas Bobik and Dr. Ted Huiatt for their help and constructive critiques and collaboration throughout my graduate career. My initial screening for the effective aptamers that can be used in DRUGINs project would not be possible without Dr. Thomas Bobik, who has kindly let me use the plate reader located in his lab. I thank Dr. Bruce Fulton for his practical training, supervision, and advice on NMR experiments. I would like to thank specially to Dr. Taner Sen who has given me the support from the first day of my PhD to the end and also for his friendship, good advice and collaboration. Also, I want to thank Julie Sen for helping me join the program.

I am grateful to my friends; Dr. Eren Kalay, Dr. Ilkay Kalay, Dr. Birkan Bayrak and Ani Anil-Bayrak for their support, care and encouragements which helped me adjust to a new environment. Also, my current and past colleagues in MNH lab; Xiaoling Song, Ilchung Shin, Supipi Liyamali Auwardt, Judhajeet Ray, Samir Mehanovic, Dr. Pierre Palo, Dr. Tianjiao Wang, Dr. Lijie Zhai and many other rotational, summer and visiting students. This inspiring group has been a constant source of friendship, good advice and collaboration.

Lastly, I would like to thank my family for their unconditional love and support during good and difficult times throughout this work. I would not have gotten this far without their care, love, understanding and constant support. I am thankful to my parents who supported 
me in all my pursuits and taught me to be independent, set goals and work hard to achieve them. This dissertation is dedicated to my wife Aliye Karabulut-llgu and particularly to my daughter Devrim Ilgaz Ilgu. I would like to thank my wife for her constant love and support during all of my PhD life as well helping me put this thesis together. Also, my mother and father, whose constant love and support, especially during the final stages of my Ph.D. is greatly appreciated. 